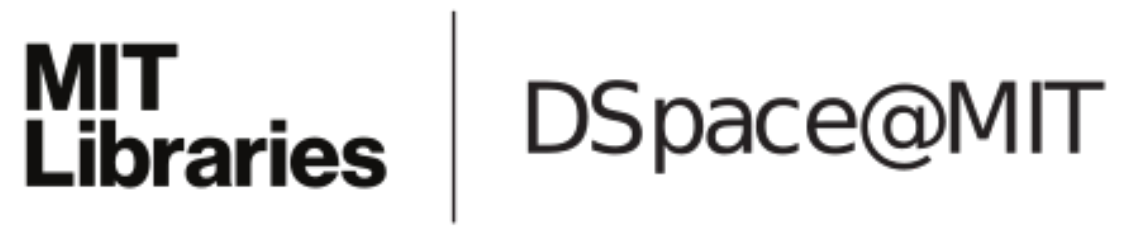

\author{
MIT Open Access Articles
}

Optics and interferometry with atoms and molecules

The MIT Faculty has made this article openly available. Please share how this access benefits you. Your story matters.

Citation: Cronin, Alexander D., Jörg Schmiedmayer, and David E. Pritchard. “Optics and interferometry with atoms and molecules." Reviews of Modern Physics 81.3 (2009): 1051. ( 2009 The American Physical Society

As Published: http://dx.doi.org/10.1103/RevModPhys.81.1051

Publisher: American Physical Society

Persistent URL: http://hdl.handle.net/1721.1/52372

Version: Final published version: final published article, as it appeared in a journal, conference proceedings, or other formally published context

Terms of Use: Article is made available in accordance with the publisher's policy and may be subject to US copyright law. Please refer to the publisher's site for terms of use. 


\title{
Optics and interferometry with atoms and molecules
}

\author{
Alexander D. Cronin* \\ Department of Physics, University of Arizona, Tucson, Arizona 85721, USA \\ Jörg Schmiedmayer ${ }^{\dagger}$ \\ Atominstitut Österreichischen Universitäten, TU-Wien, Austria \\ David E. Pritchard ${ }^{\ddagger}$ \\ Department of Physics, Massachusetts Institute of Technology, Cambridge, Massachusetts \\ 02139, USA
}

(Published 28 July 2009)

\begin{abstract}
Interference with atomic and molecular matter waves is a rich branch of atomic physics and quantum optics. It started with atom diffraction from crystal surfaces and the separated oscillatory fields technique used in atomic clocks. Atom interferometry is now reaching maturity as a powerful art with many applications in modern science. In this review the basic tools for coherent atom optics are described including diffraction by nanostructures and laser light, three-grating interferometers, and double wells on atom chips. Scientific advances in a broad range of fields that have resulted from the application of atom interferometers are reviewed. These are grouped in three categories: (i) fundamental quantum science, (ii) precision metrology, and (iii) atomic and molecular physics. Although some experiments with Bose-Einstein condensates are included, the focus of the review is on linear matter wave optics, i.e., phenomena where each single atom interferes with itself.
\end{abstract}

DOI: $10.1103 /$ RevModPhys.81.1051

PACS number(s): 03.75.Be, 03.75.Dg, 37.25.+k, 03.75.Pp

\section{CONTENTS}

I. Introduction

A. Interferometers for translational states

1052

B. Preparation, manipulation, and detection

C. Scientific promise of atom interferometers

II. Atom Diffraction

A. Early diffraction experiments

B. Nanostructures

1. Transmission gratings

2. Young's experiment with atoms

3. Charged-wire interferometer

4. Zone plates

5. Atom holography

C. Gratings of light

1. Thin gratings: Kapitza-Dirac scattering

2. Diffraction with on-resonant light

3. Thick gratings: Bragg diffraction

4. Bloch oscillations

5. Coherent channeling

D. The Talbot effect

E. Time-dependent diffraction

1. Vibrating mirrors

2. Oscillating potentials

3. Modulated light crystals

F. Summary of diffractive Atom Optics

G. Other coherent beam splitters
III. Atom Interferometers

A. Introduction

1. General design considerations

2. White light interferometetry

3. Types and categories

B. Three-grating interferometers

1. Mechanical gratings

2. Interferometers with light gratings

3. Time-domain and contrast interferometers

4. Talbot-Lau (near-field) interferometer

C. Interferometers with path-entangled states

1. Optical Ramsey-Bordé interferometers

2. Raman interferometry

D. Longitudinal interferometry

1. Stern-Gerlach interferometry

2. Spin echo

3. Longitudinal $\mathrm{rf}$ interferometry

4. Stückelberg interferometers

E. Coherent reflection

F. Confined atom interferometers with BECs

1. Interference with guided atoms

2. Coherent splitting in a double well

3. Interferometry on atom chips

IV. Fundamental Studies

A. Basic questions: How large a particle can interfere?

B. Decoherence

1. Interference and "welcher-weg" information

2. Internal state marking

3. Coupling to an environment

a. Decoherence in diffraction

b. Decoherence in Talbot-Lau interferometer

c. Photon scattering in an interferometer

d. Scattering from background gas in an

\footnotetext{
‘schmiedmayer@atomchip.org

†dpritch@mit.edu
} 


\author{
interferometer \\ 4. Realization of Feynman's gedanken \\ experiment \\ 5. Realization of Einstein's recoiling slit \\ experiment \\ C. Origins of phase shifts \\ 1. Dynamical phase shifts \\ 2. Aharonov-Bohm and Aharonov-Casher \\ effects \\ 3. Berry phase \\ 4. Inertial displacements \\ D. Extended coherence and BECs \\ 1. Atom lasers \\ 2. Studies of BEC wave functions \\ 3. Many-particle coherence in BECs \\ 4. Coupling two BECs with light \\ E. Studies with and of BECs \\ 1. Josephson oscillations \\ 2. Spontaneous decoherence and number \\ squeezing \\ 3. Structure studies of BEC \\ 4. Dynamics of coherence in 1D systems \\ 5. Measuring noise by interference \\ 6. Momentum of a photon in a medium \\ F. Testing the charge neutrality of atoms \\ V. Precision Measurements \\ A. Gravimeters, gryroscopes, and gradiometers \\ B. Newton's constant $G$ \\ C. Tests of relativity \\ D. Interferometers in orbit \\ E. Fine structure constant and $\hbar / M$ \\ VI. Atomic Physics Applications \\ A. Discovery of $\mathrm{He}_{2}$ molecules \\ B. Polarizability measurements \\ 1. Ground-state de scalar polarizability \\ 2. Transition dc and ac Stark shifts \\ C. Index of refraction due to dilute gases \\ D. Casimir-Polder (atom-surface) potentials \\ 1. vdW-modified diffraction \\ 2. Interferometer vdW and $\mathrm{CP}$ measurements \\ VII. Outlook \\ Acknowledgments \\ References \\ 1084 \\ 1085 \\ 1087 \\ 1088 \\ 1088 \\ 1089 \\ 1090 \\ 1091 \\ 1091 \\ 1091 \\ 1092 \\ 1092 \\ 1094 \\ 1095 \\ 1096 \\ 1096 \\ 1097 \\ 1097 \\ 1097 \\ 1098 \\ 1099 \\ 1099 \\ 1099 \\ 1101 \\ 1101 \\ 1102 \\ 1102 \\ 1104 \\ 1104 \\ 1104 \\ 1104 \\ 1106 \\ 1106 \\ 1107 \\ 1108 \\ 1109 \\ 1110 \\ 1114 \\ 1114
}

\section{INTRODUCTION}

Atom interferometry is the art of coherently manipulating the translational motion of atoms (and molecules) together with the scientific advances that result from applying this art. We begin by stressing that motion here refers to center of mass displacements and that coherently means with respect for (and often based on) the phase of the de Broglie wave that represents this motion. The most pervasive consequence of this coherence is interference, and the most scientifically fruitful application of this interference is in interferometers. In an interferometer atom waves are deliberately offered the option of traversing an apparatus via two or more alternate paths and the resulting interference pattern is observed and exploited for scientific gain. Atom interferometers are now valuable tools for studying fundamental quantum mechanical phenomena, probing atomic and material properties, and measuring inertial displacements.

In historical perspective, coherent atom optics is an extension of techniques that were developed for manipulating internal quantum states of atoms. Broadly speaking, at the start of the 20th century atomic beams were developed to isolate atoms from their environment; this is a requirement for maintaining quantum coherence of any sort. Hanle (1924) studied coherent superpositions of atomic internal states that lasted for tens of nanoseconds in atomic vapors. But with atomic beams, Stern-Gerlach magnets were used to select and preserve atoms in specific quantum states for several ms. A big step forward was the ability to change atoms' internal quantum states using rf resonance as demonstrated by Rabi et al. (1938). Subsequently, long-lived coherent superpositions of internal quantum states were intentionally created and detected by Ramsey (1949). The generalization and application of these techniques has created or advanced many scientific and technical fields (e.g., precise frequency standards, nuclear magnetic resonance spectroscopy, and quantum information gates).

Applying these ideas to translational motion required the development of techniques to localize atoms and transfer atoms coherently between two localities. In this view, localities in position and momentum are just another quantum mechanical degree of freedom analogous to discrete internal quantum states. We discuss these coherent atom optics techniques in Sec. II and the interferometers that result in Sec. III. Then we discuss applications for atom interferometers in Secs. IV-VI.

\section{A. Interferometers for translational states}

"Atom Optics" is so named because coherent manipulation of atomic motion requires that the atoms be treated as waves. Consequently, many techniques to control atom waves borrow seminal ideas from light optics. To make atom interferometers the following components of an optical interferometer must be replicated:

(1) state selection to localize the initial state (generally in momentum space);

(2) coherent splitting, typically using diffraction to produce at least two localized maxima of the wave function with a well-defined relative phase;

(3) free propagation so that interactions can be applied to one "arm," i.e., one of the two localized components of the wave function;

(4) coherent recombination so that phase information gets converted back into state populations;

(5) detection of a specific population, so the relative phase of the wave-function components can be determined from interference fringes. 

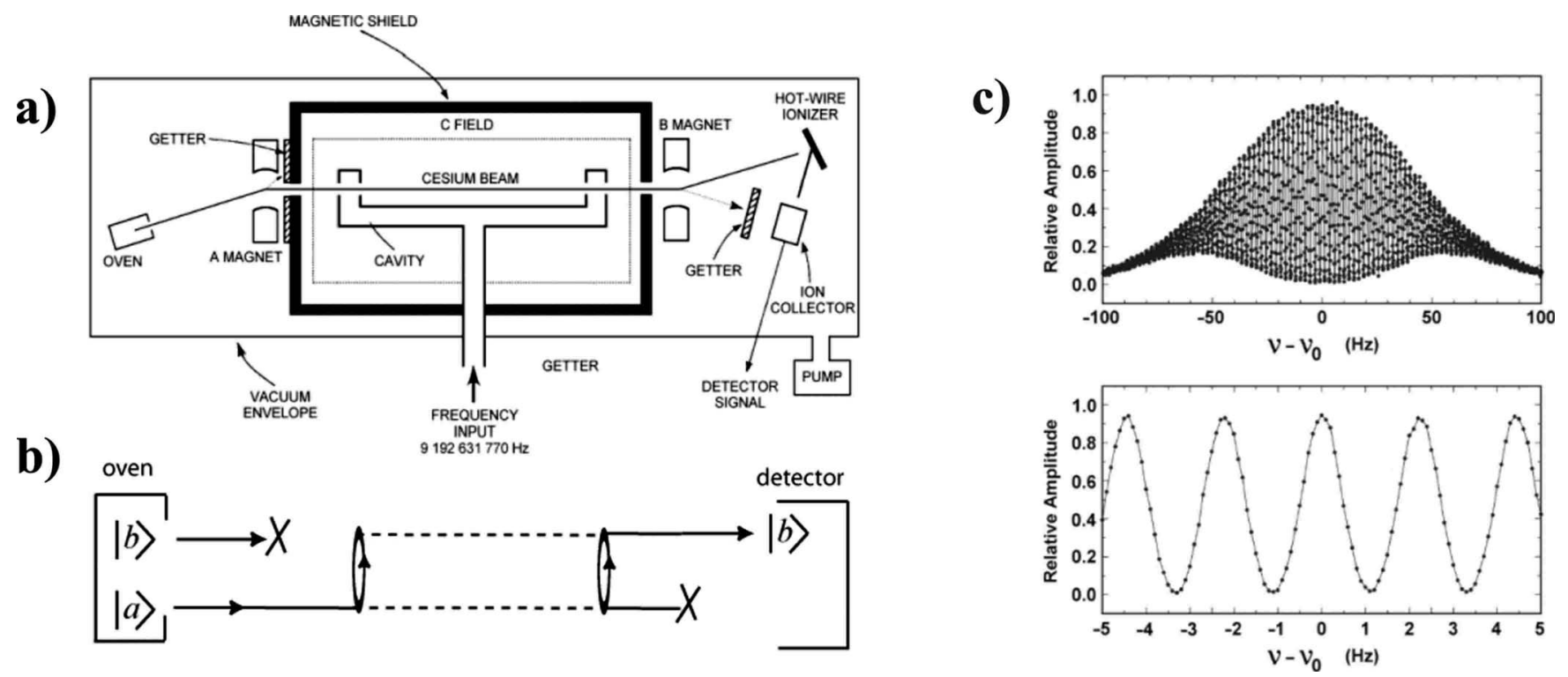

FIG. 1. Interferometry with internal quantum states of atoms. (a) Ramsey's separated oscillatory fields experiment. (b) The same experiment depicted as an interferometer for internal states. (c) The detected atom count rate exhibits interference fringes as a function of the applied rf frequency. These interference fringes, from the NIST-F1 fountain clock (Sullivan et al., 2001), demonstrate the precision obtained with interference techniques. From Sullivan et al., 2001.

In hindsight, it is possible to reinterpret much of the work on internal state resonance as an interferometer. In particular, in the separated oscillatory fields technique Ramsey (1949) divided a single rf resonance region into two zones that may be regarded as beam splitters. In this experiment a Stern-Gerlach filter (the so-called A magnet in Fig. 1) selects atoms in state $|a\rangle$. The first resonance region (microwave cavity) then excites atoms into a superposition of states $|a\rangle$ and $|b\rangle$. Atoms then travel through a static (C) field in a coherent superposition whose relative phase oscillates freely until the atoms enter the second microwave cavity. If radiation there is in phase with the oscillating superposition, then atoms complete the transition to state $|b\rangle$. But if the radiation is half a cycle out of phase, then atoms are returned to state $|a\rangle$. After the final state selector, the detected intensity oscillates as a function of microwave frequency. Overall, this method to manipulate the internal states of an atom obviously maps directly onto the steps listed above and can be regarded as the first atom interferometer even though it is more frequently described in terms of resonance of a Bloch vector of an atom moving classically.

\section{B. Preparation, manipulation, and detection}

Preparation of position states is hindered by the uncertainty principle. As soon as free atoms are localized in position, the attendant momentum uncertainty starts to cause spatial delocalization. On the other hand, preparation in momentum space is free of such back action. Therefore in coherent atom optics, especially with free atoms, it is desirable to reduce the momentum and its uncertainty for an ensemble of atoms. This is referred to as slowing and cooling the atoms, respectively.

Momentum-state selection can be as simple as two collimating slits that select atoms with limited transverse momentum. Alternatively, and preferably, atoms can be concentrated in phase space by laser cooling and trapping. ${ }^{1}$ This is analogous to optical pumping for internal states. In fact, cooling atoms (or ions) in a trap is even more exactly analogous to optical pumping because trapped atoms are in discrete translational states and can ultimately be prepared in the single ground state.

The typical momentum uncertainty achieved with various methods is summarized in Table I. We note that atom interferometers already work with atoms prepared in beams, magneto-optical traps, or Bose-Einstein condensates.

Manipulation. In most atom interferometers diffraction or the closely related Raman transitions "split" atoms into a coherent superposition of momentum states that typically differ in momentum by several photon momenta (velocity differences of several $\mathrm{cm} / \mathrm{s}$; e.g., the re-

\footnotetext{
${ }^{1}$ Original references for cooling and trapping include supersonic beams (Beijerinck and Verster, 1981; Campargue, 1984), optical molasses (Chu et al., 1985; Aspect et al., 1986), optical traps (Ashkin, 1970; Chu, Bjorkholm, Ashkin, and Cable, 1986; Chu, Bjorkholm, Ashkin, Gordon, et al., 1986; Miller et al., 1993), magneto-optical traps (Raab et al., 1987), magnetic traps (Pritchard, 1983; Migdall et al., 1985), atomic fountains (Kasevich et al., 1989), velocity selective coherent population trapping (Aspect et al., 1988), sideband cooling (Neuhauser et al., 1978; Wineland et al., 1978; Vuletic et al., 1998), cooling to the ground state of a trap (Jessen et al., 1992; Monroe et al., 1995), and Bose-Einstein condensation (Anderson et al., 1995).
} 
TABLE I. Momentum uncertainty and temperature of atoms prepared with different techniques. Typical best case values for sodium atoms are tabulated. The momentum uncertainty $\sigma_{p}$ $=\left(\left\langle p^{2}\right\rangle-\langle p\rangle^{2}\right)^{1 / 2}$ is given in units of 590-nm photon momenta $\hbar k_{\mathrm{ph}}$. Temperature is given by $k_{B} T=\sigma_{p}^{2} / 2 m$, where $k_{B}$ is the Boltzmann constant and $m$ is the atomic mass.

\begin{tabular}{lcc}
\hline \hline Atomic sample & $\sigma_{p} / \hbar k_{\mathrm{ph}}$ & $T(\mathrm{~K})$ \\
\hline Thermal vapor & 24000 & 500 \\
Effusive beam (longitudinal) & 8000 & 50 \\
Supersonic beam (longitudinal) & 3000 & 8 \\
Optical molasses or MOT & 20 & 0.00025 \\
Collimated beam (transverse) & 1 & $10^{-6}$ \\
Bose-Einstein condensate & 0.1 & $10^{-8}$ \\
\hline \hline
\end{tabular}

coil velocity for $\mathrm{Na}$ atoms due to absorbing a $590-\mathrm{nm}$ photon is $v_{\mathrm{rec}}=\hbar \mathrm{k} / \mathrm{m}_{\mathrm{Na}}=2.9 \mathrm{~cm} / \mathrm{s}$ and the velocity difference between 0th and 1st diffraction orders for $\mathrm{Na}$ atoms transmitted through $100-\mathrm{nm}$ period gratings is $\left.h / m_{\mathrm{Na}} d=17 \mathrm{~cm} / \mathrm{s}\right)$. As time passes, each atom evolves into a coherent superposition of spatial positions located a distance $\Delta x=\left(p_{2}-p_{1}\right) t / m$ apart. Moreover, if the initial preparation was restrictive enough, then the components of each atoms' wave function will be distinctly separated in space. Creating such "separated beams" in an interferometer invites the experimenter to deliberately apply different interactions-and hence different phase shifts-to each component of an atom's wave function.

Observing this phase difference requires recombining the two components of the superposition. This is generally achieved using diffraction or Raman processes again to reverse the momenta of the two states so they subsequently overlap. When this is done, interference fringes are observed and the phase $\phi_{\text {int }}$ can be determined from their position.

Detection. Once information is transferred from the phase of a superposition into the population of observable states using some kind of beam recombiner, then a state-selective detector is used to measure the output of an interferometer. In analogy with an optical MachZehnder interferometer, the fringes can be observed as atom beam intensity that oscillates between two "output" momentum states as a function of the interactioninduced phase difference $\phi_{\text {int }}$. Alternatively, fringes can be observed directly in position space either by moiré filtering with a suitable mask or by directly imaging the atoms. Bragg reflection of laser light can also be used to detect fringes in atomic density. If the interferometer manipulates both the internal and (separated) external states of atoms, then fringes can be detected as oscillations in population of the internal states after recombining the atoms, as in Ramsey's experiment.

Historically, alkali-metal atoms were the first to be detected efficiently, and this was achieved by counting the ions produced as the atoms ionized on a hot tungsten or rhenium wire. ${ }^{2}$ Metastable atoms can be detected directly with multichannel plates because of their stored internal energy. More universal neutral atom detectors use electron bombardment or laser excitation to produce countable ions. Fluorescence or absorption can also reveal fringes, especially if a cycling transition is used with slow atoms.

\section{Scientific promise of atom interferometers}

The light interferometers that were developed late in the 19th century by Fizeau (1853), Michelson (1881), Rayleigh (1881), and Fabry and Perot (1899) performed many experiments and precise measurements that have had a broad impact in physics. Recently, the initial idea from de Broglie and Schrödinger that propagating particles are waves has been combined with technologies to produce interferometers for electrons (Marton et al. 1953, 1954) neutrons (Rauch et al., 1974), and now atoms. Even after the many advances made possible with earlier interferometers, further scientific advances from atom interferometers have long been anticipated. In fact, the concept of an atom interferometer was patented by Altschuler and Franz (1973) and it has been extensively discussed since. Early proposals for atom interferometers were made by Chebotayev et al. (1985), Clauser (1988, 1989), Keith et al. (1988), Martin et al. (1988), Bordé (1989), Pritchard (1989), and Kasevich and Chu (1991).

Even compared to electron- and neutron-wave physics, interferometry with atoms offers advantages on several fronts: a wider selection of atomic properties, larger cross sections for scattering light, better characterized environmental interactions, higher precision, better portability, and far lower cost. Atomic properties like mass, magnetic moment, and polarizability can be selected over ranges of several orders of magnitude. For example, Cs has 137 times the mass and 89 times the electric polarizability of $\mathrm{H}$ and is therefore better suited to measuring inertial effects and detecting weak electric fields. ${ }^{52} \mathrm{Cr}$ has a magnetic moment of $6 \mu_{B}$ while ${ }^{4} \mathrm{He}$ has none. Alkali-metal atoms have $10^{-9}-\mathrm{cm}^{2}$ scattering cross sections for resonant light while electrons have a $10^{-25}-\mathrm{cm}^{2}$ cross section for the same light (Compton or Thomson scattering). Hence interactions of atoms and their environment can be enlarged for better measurements or to study decoherence, or they can be suppressed to measure something else. Furthermore, atoms interact with surfaces and other atomic gases with potentials that are easily studied by interferometry. Atoms can be manipulated by lasers whose frequency and wavelength are measured with accuracies of $10^{-15}$ and $10^{-11}$, respectively, offering far better precision for mea-

\footnotetext{
${ }^{2}$ Various atom detectors have been discussed by Ramsey (1985), Scoles (1988), and Campargue (2000). For hot wire detectors see Langmuir and Kingdon (1925), and Delhuille, Miffre, et al. (2002); for universal detectors see Kuhnke et al. (1994) and DeKieviet, Dubbers, Klein, et al. (2000).
} 
surements than the crystals or structures used in other types of interferometers. Finally, atom sources can be as simple as a heated container with a small hole in the side or a pulse of laser light that hits a pellet of the desired material. These sources are far less expensive than nuclear reactors or even $200-\mathrm{keV}$ electron guns. In fact, atom interferometers on atom chips can potentially fit in a briefcase.

This richness and versatility is combined with the rewards (and challenges) that stem from the fact that thermal atomic wavelengths are typically 30000 times smaller than wavelengths for visible light. The power of atom interferometry is that we can measure phase shifts $\phi_{\text {int }}=\hbar^{-1} \int U d t$ due to very small potential energies. A simple calculation shows that $1000-\mathrm{m} / \mathrm{s}$ Na atoms $\left(E_{\text {kin }}\right.$ $\sim 0.1 \mathrm{eV}$ ) acquire a phase shift of $1 \mathrm{rad}$ for a potential of only $U=6.6 \times 10^{-12} \mathrm{eV}$ in a $10-\mathrm{cm}$ interaction region. Such an applied potential corresponds to a refractive index of $|n-1|=2.7 \times 10^{-11}$. Measuring the phase shift $\phi_{\text {int }}$ to $10^{-3}$ rad corresponds to an energy resolution of $U / E \sim 10^{-14}$, or a spectrometer with a linewidth of $10 \mathrm{kHz}$ and spectroscopic precision of $\mathrm{Hz} / \sqrt{\mathrm{s}}$. Furthermore, this example was for a thermal atomic beam; cold atoms can increase the sensitivity 1000-fold.

As we document in this review, atom interferometers have already measured rotations, gravity, atomic polarizability, the fine structure constant, and atom-surface interactions better than previous methods. Yet atom interferometry itself is just over a decade old. The realization of such interferometers started with diffraction gratings that are summarized in Sec. II. We catalog atom interferometer types and features in Sec III. We discuss fundamental issues such as decoherence in Sec. IV. Precision measurements are described in Sec. V, and atomic and molecular physics applications are described in Sec. VI.

\section{ATOM DIFFRACTION}

Since half-silvered mirrors do not exist for atoms (solid matter generally absorbs or scatters atoms), beam splitters for atom interferometers are often based on diffraction. Diffraction itself is an interesting interference effect that has already been developed for use with atoms. Hence we discuss atom diffraction now, and atom interferometers next (in Sec. III).

Diffraction occurs when a wave interacts with anything that locally shifts its phase or amplitude (e.g., due to absorption), and is a hallmark of wave propagation and interference. It is generally treated as resulting from the coherent superposition and interference of amplitudes for wave propagation via different paths through the diffracting region that have the same starting and ending points.

A diffraction grating is a periodic diffracting region. Spatial modulation of the wave by the grating generates multiple momentum components for the scattered waves. The fundamental relationship between the mo- mentum transferred to waves in the $n$th component and the grating period $d$ is

$$
\delta p_{n}=n h / d=n \hbar G
$$

where $G=2 \pi / d$ is the reciprocal lattice vector of the grating and $h$ is Planck's constant. When the incoming wave has a narrow transverse momentum distribution centered around $p_{\text {beam }}$, this diffraction is generally observed with respect to angle. Since the de Broglie wavelength is $\lambda_{\mathrm{dB}}=h / p_{\text {beam }}$ (de Broglie, 1923), the resulting diffraction angles (for nearly normal incidence) are

$$
\theta_{n} \approx \delta p_{n} / p_{\text {beam }}=n \lambda_{\mathrm{dB}} / d \text {. }
$$

To observe the interference a grating must be illuminated at least in part coherently, i.e., the incident atom waves must have a well-defined relative phase across several grating periods. That means the transverse coherence length must be larger than a few grating periods, i.e., the transverse momentum distribution must be small enough to resolve the diffraction orders. This is usually accomplished by collimating the incident beam. ${ }^{3}$

\section{A. Early diffraction experiments}

The first examples of atom interference were diffraction experiments, and the earliest of these was by Estermann and Stern (1930) just three years after the electron diffraction experiment by Davisson and Germer (1927). Figure 2 shows original data in which helium atoms were reflected and diffracted from the surface of a LiF crystal. The small lattice period of the crystal surface $(40 \mathrm{~nm})$ gave large diffraction angles and allowed relaxed collimation. This observation proved that composite particles (atoms) propagate as waves, but this kind of reflection-type diffraction grating has not led to a beam splitter suitable for atom interferometry. It did, however, launch an active field of atom diffraction (both elastic and inelastic) for studying surfaces.

\section{B. Nanostructures}

One of the first demonstrations of atom diffraction from macroscopic objects was by Leavitt and Bills (1969) who observed Fresnel diffraction from a single $20-\mu \mathrm{m}$-wide slit. With the advent of modern nanotechnology it became possible to fabricate elaborate arrays of holes and slots in a thin membrane that allow atoms to pass through. These can have feature sizes of $50 \mathrm{~nm}$-much smaller then typical transverse coherence in well-collimated atomic beams. Diffraction from a nanofabricated structure-a transmission grating with 200-nm-wide slits-was first observed by the Pritchard

\footnotetext{
${ }^{3}$ The transverse coherence length is $\ell_{\text {tcoh }} \approx \lambda_{\mathrm{dB}} / \vartheta_{\text {coll }}$, where $\lambda_{\mathrm{dB}}$ is the de Broglie wavelength and $\vartheta_{\text {coll }}$ is the (local) collimation angle of the beam (the angle subtended by a collimating slit). Since for thermal atomic beams $\lambda_{\mathrm{dB}} \sim 10 \mathrm{pm}$ a collimation of $\vartheta_{\text {coll }}<10 \mu \mathrm{rad}$ is required for a $1-\mu \mathrm{m}$ coherent illumination.
} 


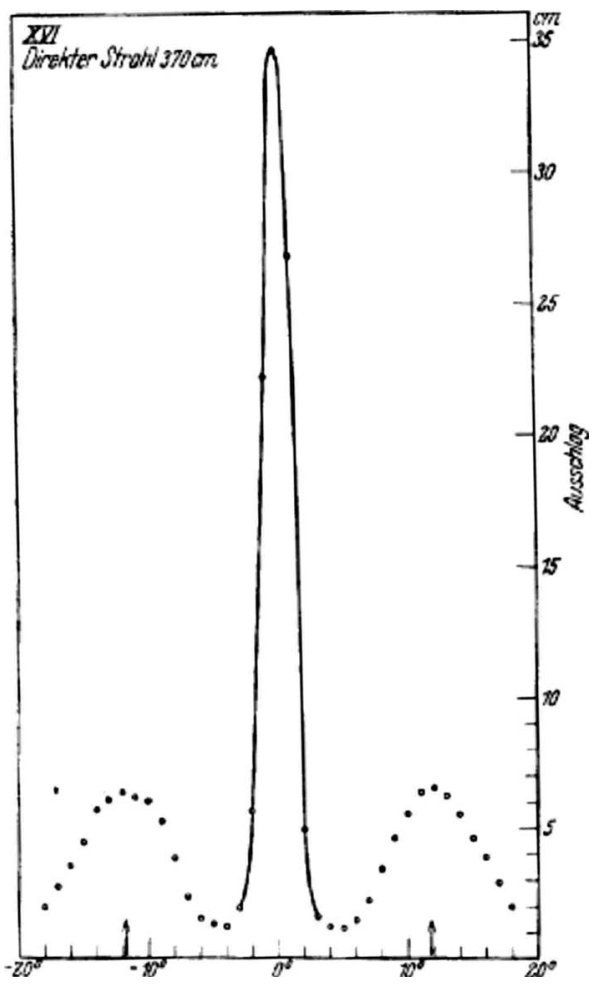

FIG. 2. Historic data showing diffraction of He atoms from a $\mathrm{LiF}$ crystal surface. The central peak is due to He atom reflection. The side peaks are due to first-order diffraction of $\mathrm{He}$ atoms from the LiF crystal lattice. From Estermann and Stern, 1930.

group at MIT (Keith et al., 1988). This led to many interference experiments with atoms and molecules.

Nanotechnology has been used to make single slits, double slits, diffraction gratings, zone plates, hologram masks, mirrors, and phase shifting elements for atom waves. The benefits of using mechanical structures for atom optics include feature sizes smaller than light wavelengths, arbitrary patterns, rugged designs, and the ability to diffract any atom and/or molecule. The primary disadvantage is that atoms stick to (or bounce back from) surfaces, so that most structures serve as absorptive atom optics with a corresponding loss of transmitted intensity.

\section{Transmission gratings}

After the demonstration of transmission gratings for atom waves by Keith et al. (1988), these gratings have seen numerous applications. A $100-\mathrm{nm}$ period nanostructure grating made at the MIT NanoStructures facility and atom diffraction data from this kind of grating is shown in Fig. 3. Material structures absorb atoms that hit the grating bars but transmit atom waves through the slots relatively unperturbed.

Classical wave optics recognizes two limiting cases, near and far field, treated by the Fresnel and Fraunhoffer approximations, respectively. Both regimes have revealed interesting effects and led to scientific advance. In the near-field limit the curvature of the wave fronts

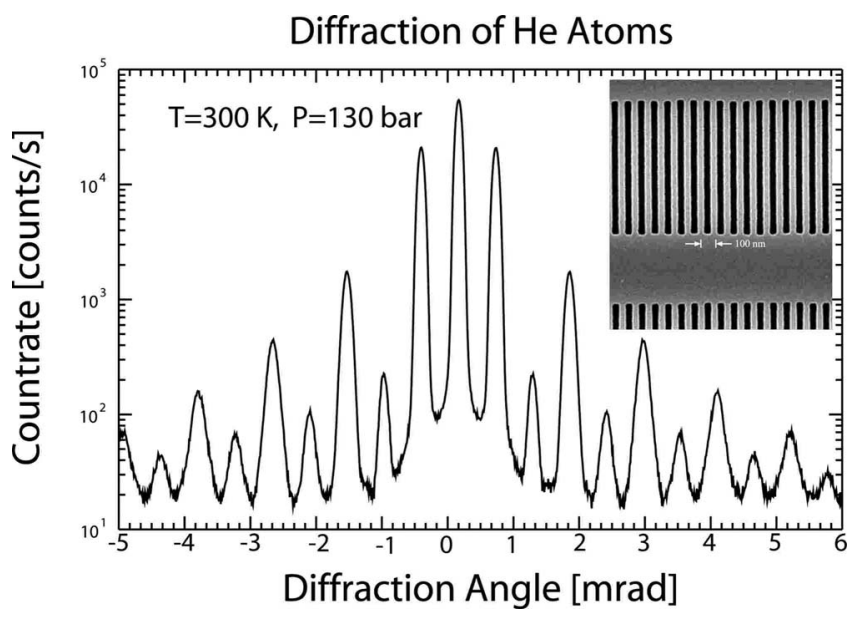

FIG. 3. Diffraction of He atoms transmitted through a nanostructure grating. The average velocity and velocity spread of the beam, the uniformity of the material grating, and the strength of atom-surface van der Waals forces can all be determined from these data (Grisenti et al., 1999). From J. P. Toennies, W. Schöllkopf, and O. Kornilov. Inset: A 100-nm-period grating for atom waves. The dark regions are slots, and light regions are free-standing silicon nitride bars. From T. A. Savas and H. I. Smith at the MIT NanoStructure Laboratory (Schattenburg et al., 1990; Savas et al., 1995, 1996).

must be considered and the intensity pattern of the beam is characterized by Fresnel diffraction. Edge diffraction and the Talbot self-imaging of periodic structures are examples of near-field atom optics. In the farfield limit, the intensity pattern of the beam is characterized by Fraunhofer diffraction in which the curvature of the atom wave fronts is negligible and the diffraction orders can be resolved. For a grating with open fraction $w / d$ and a purely real and binary valued transmission function the probability for a beam to be diffracted into the $n$th order is

$$
P_{n}=I_{n} / I_{\mathrm{inc}}=(w / d)^{2}\left[\frac{\sin (n w \pi / d)}{n w \pi / d}\right]^{2} .
$$

Modification of the diffraction patterns due to van der Waals interaction with the grating bars was first observed by Grisenti et al. (1999). This reduces the flux in the zeroth order, increases flux in most of the higher orders and prevents "missing orders" from occurring (Cronin and Perreault, 2004). Random variations in the grating bar period can be analyzed as Debye-Waller damping which preferentially suppresses higher diffraction orders (Grisenti, Schöllkopf, Tonnies, Manson, et al. 2000). Molecular size effects also modify the relative efficiencies as described by Grisenti, Schöllkopf, Toennies, Hegerfeldt, et al. (2000) and were used to estimate the size of the very weakly bound $\mathrm{He}_{2}$ molecule (Luo et al., 1993; Schöllkopf and Toennies, 1994, 1996).

Molecules such as ${ }^{4} \mathrm{He}_{2},{ }^{4} \mathrm{He}_{3}$, and other ${ }^{4} \mathrm{He}$ clusters, $\mathrm{Na}_{2}, \mathrm{C}_{60}, \mathrm{C}_{70}, \mathrm{C}_{60} \mathrm{~F}_{48}$, and $\mathrm{C}_{44} \mathrm{H}_{30} \mathrm{~N}_{4}$ have been diffracted from similar gratings (Chapman, Ekstrom, Hammond, Rubenstein, et al., 1995; Schöllkopf and Toennies, 1996; Arndt et al., 1999; Brezger et al., 2002, 2003; Hacker- 

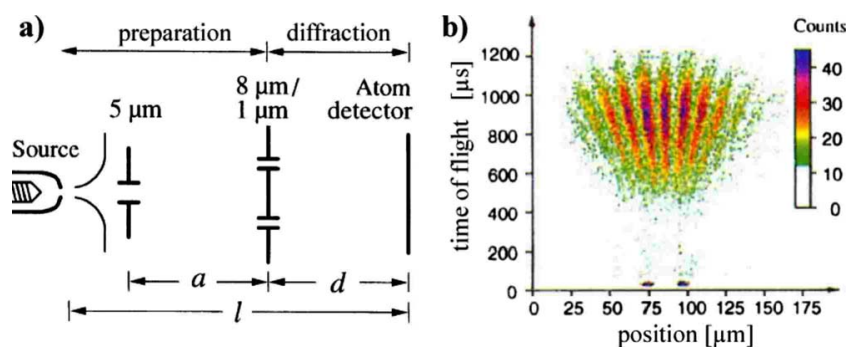

FIG. 4. (Color online) Double-slit experiment with $\mathrm{He}^{*}$. (a) Schematic. (b) Atom interference pattern with $a=1.05 \mathrm{~m}$ and $d=1.95 \mathrm{~m}$ recorded with a pulsed source. Adapted from Kurtsiefer et al., 1997.

muller, Uttenthaler, et al., 2003; Nairz et al., 2003; Bruehl et al., 2004). Scientific results in this area, such as the study of decoherence and the formation rate of molecules in beams, will be discussed in Sec. IV.

\section{Young's experiment with atoms}

Atomic diffraction from a double slit recapitulates the seminal Young's double slit experiment in which the diffraction pattern is created by the interference of waves traversing two cleanly separated paths. In that sense it can be seen as a two path interferometer. The atomic version by Carnal and Mlynek (1991) used a mechanical structure with two $1-\mu \mathrm{m}$-wide slits separated by $8 \mu \mathrm{m}$ to create the interference (Fig. 4). Diffraction from a single $2-\mu \mathrm{m}$-wide slit $62 \mathrm{~cm}$ from the double slit prepared the atom waves $\left(\lambda_{\mathrm{dB}}=100 \mathrm{pm}\right)$ to have a transverse coherence length larger than the double slit separation $\left(\ell_{t c o h}\right.$ $\left.=z \lambda_{\mathrm{dB}} / 2 w=15 \mu \mathrm{m}\right)$.

In the original experiment, a slit was translated in front of the detector to observe the interference fringes. With a beam brightness of $B \approx 10^{17} \mathrm{~s}^{-1} \mathrm{~cm}^{-2} \mathrm{sr}^{-1}$, the average count rate was about one atom per second. In a later version (see Fig. 4) they used a position sensitive detector to record the whole pattern at once, giving a larger counting rate. Time of flight resolution was added in order to measure the Wigner function of the transmitted atoms (Kurtsiefer et al., 1997).

A two slit experiment using cold $\mathrm{Ne}^{*}$ atoms was presented by Shimizu et al. (1992). The atoms were dropped from a magneto-optical trap $1 \mathrm{~m}$ above a mechanical mask with two slits separated by $6 \mu \mathrm{m}$. At the location of the mask the atoms had a speed of $4.5 \mathrm{~m} / \mathrm{s}\left(\lambda_{\mathrm{dB}}\right.$ $=5 \mathrm{~nm})$ and a speed ratio of $v / \sigma_{v}=20$. The mask was equipped with an electrode so that deflection due to an applied electric field gradient could be measured.

\section{Charged-wire interferometer}

A variation of the atomic Young's experiment was built by Nowak et al. (1998). A single wire put in a He* beam produces a near-field diffraction pattern. Charging the wire bends the atom trajectories passing around it inward, increasing the interference (Fig. 5) analogous to the charged-wire optical bi-prism interferometer for electrons (Mollenstedt and Duker, 1955).

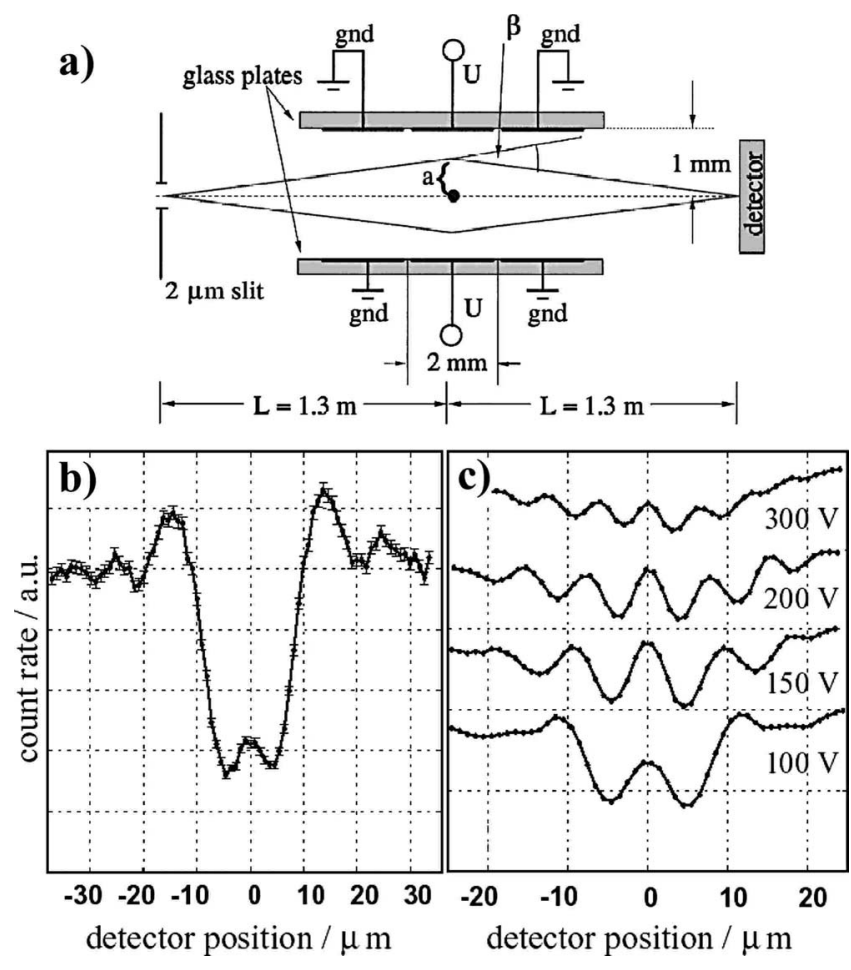

FIG. 5. Charged wire interferometer. (a) Schematic. (b) Measured diffraction patterns with an uncharged wire. Fresnel fringes and the Poisson spot are visible. (c) Interference fringes with different voltages applied to the electrodes. Adapted from Nowak et al., 1998.

\section{Zone plates}

Fresnel zone plates have focused atoms to spots smaller than $2 \mu \mathrm{m}$ (Fig. 6). Zone plates behave locally like a diffraction grating, therefore the focal length of a zone plate is $f=R d_{\min } / \lambda_{\mathrm{dB}}$, where $R$ is the radius of out-

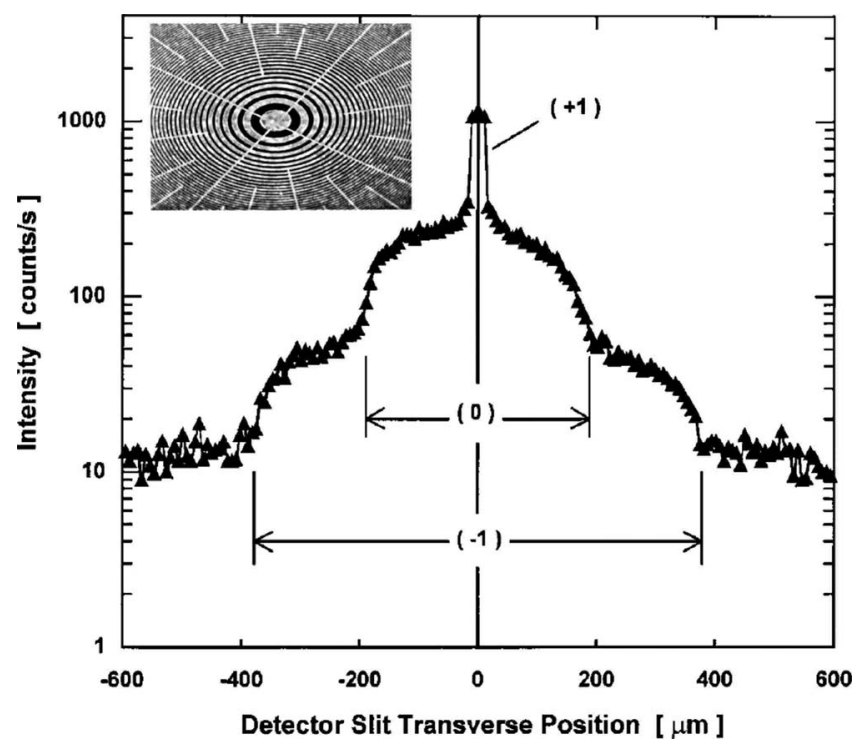

FIG. 6. A zone plate for focusing atom beams. The plate (inset) has free-standing annular rings and radial support struts. The data show focused $(+1)$ and defocused $(-1)$ atom beam components. Adapted from Doak et al. 1999. 


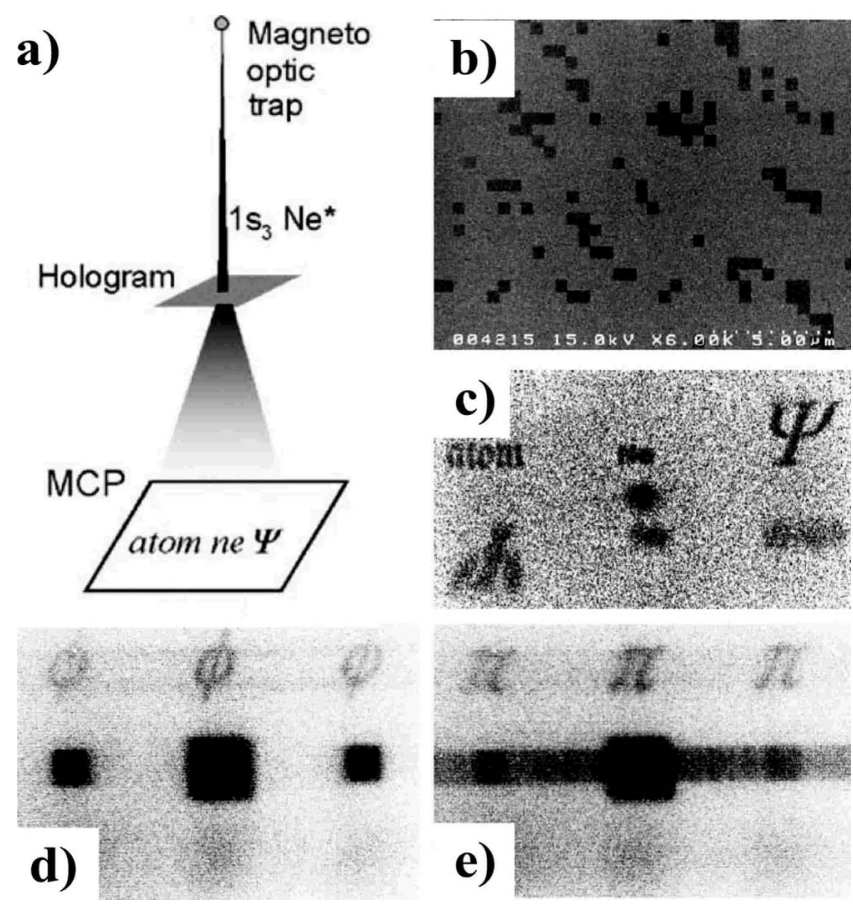

FIG. 7. Atom holography. (a) Experimental setup for image reconstruction of the hologram by an atom beam. (b) A hologram designed by computer and realized with a $\mathrm{SiN}$ membrane with square holes. (c) Far-field diffraction pattern from the hologram mask. (d), (e) Two different diffraction patterns obtained with a switchable hologram. Adapted from Fujita et al., 2000b and Morinaga, Yasuda, et al., 1996; Fujita et al., 2000a.

ermost zone and $d_{\min }$ is the period of the smallest features. Focal lengths of $f=450 \mathrm{~mm}$ with $R=0.2 \mathrm{~mm}\left(\lambda_{\mathrm{dB}}\right.$ $=200 \mathrm{pm})$ (Carnal et al., 1999) and $f=150 \mathrm{~mm}$ with $R$ $=0.3 \mathrm{~mm}\left(\lambda_{\mathrm{dB}}=180 \mathrm{pm}\right)$ (Doak et al., 1999) have been demonstrated.

\section{Atom holography}

Atom holography with nanostructures can make the far-field atom flux display arbitrary patterns. Adding electrodes to a structure allows electric and magnetic fields that cause adjustable phase shifts for the transmitted atom waves. With this technique, Fujita et al. (1996, 1999, 2000a) demonstrated a two-state atom holographic structure that produced images of the letters $\phi$ or $\pi$ as shown in Fig. 7. The different holographic diffraction patterns are generated depending on the voltages applied to each nanoscale aperture.

\section{Gratings of light}

Laser spectroscopy initially dealt with the internal energy levels of atoms, and coherent phenomena such as nonlinear optics. Exploiting the momentum transfer accompanying absorption or emission of light was of little experimental concern until the observation of quantized deflection (diffraction) in an atom beam by Moskowitz et al. (1983) and Pritchard and its subsequent application to a BEC by Ovchinnikov et al. (1999). ${ }^{4}$ Many experiments with atoms diffracted from standing waves of light have been accomplished since these earliest milestones (see, e.g., Martin et al., 1988; Giltner et al., 1995a, 1995b; Rasel et al., 1995; Stenger et al., 1999; Torii et al., 2000; Delhuille, Champenois, et al., 2002; Koolen et al., 2002). Now the interaction between light and atoms is recognized as a rich resource for atom diffraction (and interference) experiments; and a unified view of all possible atom diffraction processes using light beams has been presented by Bordé (1997). Light waves can act as refractive, reflective, and absorptive structures for matter waves, just as glass interacts with light waves.

In an open two-level system the interaction between an atom and the light field (with detuning $\Delta=\omega_{\text {laser }}$ $\left.-\omega_{\text {atom }}\right)$ can be described with an effective optical potential of the form (Oberthaler, Abfalterer, et al., 1996)

$$
U(x)=\hbar \Omega_{1}^{2} /(4 \Delta+i 2 \Gamma) \propto I(x) /(2 \Delta+i \Gamma),
$$

where the (on-resonant) Rabi frequency, $\Omega_{1}$ $=\mathbf{d}_{\mathrm{ab}} \cdot \mathbf{E}_{\text {optical }} / \hbar$, is given by the atomic transition dipole moment and the optical electric field, $\Gamma$ is the atomic decay rate, and $I(x)$ is the light intensity. The imaginary part of the potential comes from the spontaneous scattering processes, and the real part results from the ac Stark shift. For a more detailed description we point the reader to the vast literature on mechanical effects of light. ${ }^{5}$ If the spontaneous decay follows a path to a state which is not detected, the imaginary part of the potential in Eq. (4) is equivalent to absorption. Therefore onresonant light can be used to create absorptive structures. Light with large detuning produces a nearly real potential and therefore acts as a pure phase object. Near-resonant light can have both roles.

The spatial shape of the potential is given by the local light intensity pattern $I(x)$, which can be shaped with the near- and far-field optics for light, including holography. The simplest object is a periodic potential created by two beams of light whose interference forms a standing wave with reciprocal lattice vector

$$
\vec{G}=\vec{k}_{1}-\vec{k}_{2} \text {. }
$$

This is often called an optical lattice because it is a close realization of the periodic potentials that electrons experience in solid state crystals. Thus Bloch states can be used to understand atom diffraction (Letokhov et al., 1981; Champenois et al., 2001a) Additional points of view that we discuss include the thin hologram (RamanNath approximation) (Meystre, 2001), two-photon Rabi oscillations (Gupta, Leanhardt, et al., 2001) and multibeam interference (dynamical diffraction theory).

We distinguish different regimes for atom manipulation, for example, (i) thick versus thin optical lattices, (ii)

\footnotetext{
${ }^{4}$ Theoretical work on quantized momentum transfer from light to matter dates back to Einstein (1917) and Kapitza and Dirac (1933).

${ }^{5}$ See, for example, Ashkin (1970, 1980); Dalibard and CohenTannoudji (1985); Metcalf and van der Stratten (1999).
} 


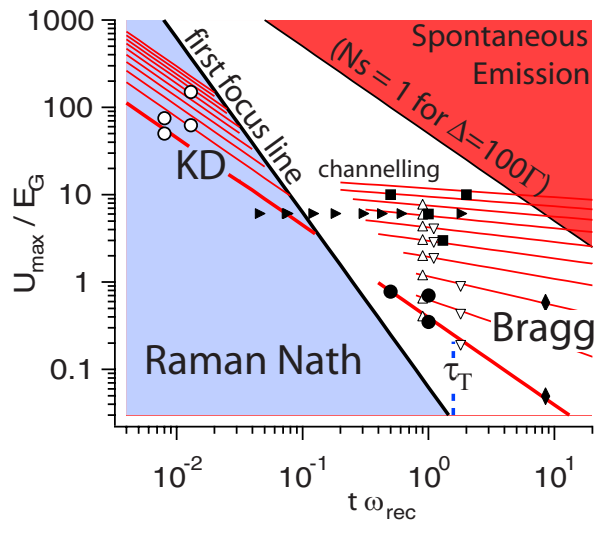

FIG. 8. (Color online) Dimensionless parameter space for atom diffraction. The vertical axis (optical potential in units of $E_{G}$ ) and horizontal axis (interaction time in units of $\omega_{\text {rec }}^{-1}$ ) are independent of atomic transition dipole moment and atomic mass [see Eqs. (4) and (6)]. Under the first focus line the RNA [Eq. (8)] is satisfied. KD labels curves corresponding to conditions that maximize Kapitza-Dirac diffraction into orders 1-10 in order from bottom to top [see Eq. (7)]. Bragg indicates curves that correspond to conditions for complete ( $\pi$ pulse) Bragg reflection into orders 1-10 [see Eqs. (11) and (12)]. The vertical dashed line indicates the Talbot time $\tau_{T}$ (discussed in Sec. II.D). For detuning of $\Delta=100 \Gamma$, the average number of spontaneously scattered photons per atom is greater than 1 above the line marked $N_{s}=1$. Experiment conditions are shown as points. $\bigcirc$, Kapitza-Dirac diffraction of an atomic beam (Gould et al., 1986). ๑, $\triangle, \nabla$, Bragg diffraction of an atomic beam [Martin et al. (1988), Giltner et al. (1995a), Koolen et al. (2002), respectively]. $\bullet$, Bragg diffraction of a BEC (Kozuma, Deng, et al., 1999) [Bragg spectroscopy of a BEC (Stenger et al., 1999) at $\tau \omega_{\text {rec }}=80$ would appear to the right of the charted regions, near the first-order Bragg curve]. $\checkmark$, Transition from Kapitza-Dirac diffraction to oscillation of a BEC in a standing wave light pulse (Ovchinnikov et al., 1999)

口, Coherent channeling. Adapted from Keller et al., 1999, and Gupta, Leanhardt, et al., 2001.

weakly perturbing versus strongly channeling lattices, (iii) on-versus off-resonant light, and (iv) static versus time-dependent optical potentials. These have been discussed by Oberthaler, Abfalterer, et al. (1996); Oberthaler et al. (1999); Keller et al. (1999); Bernet et al. (2000); Champenois et al. (2001a); Gupta, Leanhardt, et al. (2001); Morsch and Oberthaler (2006). We include a summary chart (Fig. 8) that catalog different effects caused by gratings of light.

Since light gratings can fill space, they can function as either thin or thick optical elements. As in light optics, for a thin optical element the extent of the grating along the propagation direction has no influence on the final diffraction (interference). But in a thick element the full propagation of the wave throughout the diffracting structure must be considered. In a grating, the relevant scale is set by the grating period $(d)$ and the atomic de Broglie wavelength $\left(\lambda_{\mathrm{dB}}\right)$. If a grating is thicker than $d^{2} / \lambda_{\mathrm{dB}}$ (half the Talbot length) it is considered thick, and the characteristics observed are Bragg scattering or channeling, depending on the height of the potentials. If the grating is thinner than $d^{2} / \lambda_{\mathrm{dB}}$, it can be analyzed in the Raman-Nath limit, and it produces a symmetric distribution of intensity into each pair of diffraction orders of opposite sign $( \pm N)$. The thin versus thick transition is labeled first focus line in Fig. 8.

The second distinction, mostly relevant for thick gratings, has to do with the strength of the potential. One must determine if the potential is only a perturbation, or if the potential modulations are larger then the typical transverse energy scale of the atomic beam or the characteristic energy scale of the grating,

$$
E_{G}=\hbar^{2} G^{2} /(2 m)=4 \hbar \omega_{\text {rec }} .
$$

associated with one grating momentum unit $\hbar G . \hbar \omega_{\text {rec }}$ is an atoms "recoil energy" due to absorbing (or emitting) a photon. For weak potentials, $U \ll E_{G}$, one observes Bragg scattering. The dispersion relation looks like that of a free particle with avoided crossings at the edges of the zone boundaries. Strong potentials, with $U \gg E_{G}$, cause channeling. The dispersion relations are nearly flat, and atoms are tightly bound in the wells.

\section{Thin gratings: Kapitza-Dirac scattering}

If atoms are exposed to a standing wave of offresonant light for a short time $\tau$, the resulting optical potential due to the standing wave acts as a thin phase grating with period $d=\lambda_{\mathrm{ph}} / 2$. Atom waves are diffracted by this grating so that many momentum states (each differing by $\hbar G$ ) are populated as shown in Fig. 9 (left column). This is known as Kapitza-Dirac scattering, ${ }^{6}$ and occurs in the Raman-Nath limit. The probability of finding atoms in the Nth diffracted state is given by the Fourier transform of the imprinted phase shift, resulting in Gupta, Leanhardt, et al., 2001)

$$
P_{N}^{\mathrm{KD} \text { thin }}=J_{N}^{2}\left(\Omega_{1}^{2} \tau / 2 \Delta\right) .
$$

Here $J_{N}$ is an $N$ th-order Bessel function and $\tau$ is the duration that the optical intensity is experienced by the atoms. As defined near Eq. (4), $\Omega_{1}=\mathbf{d}_{\mathrm{ab}} \cdot \mathbf{E}_{\text {optical }} / \hbar$. Equation (7) is valid for normal incidence; Henkel et al. (1994) considered all angles of incidence.

The Raman-Nath approximation (RNA) is valid provided the transverse motion of the atoms remains small. Approximating the potential from a standing wave as parabolic near a the minimum leads to the condition for the RNA to be valid:

$$
\tau<\tau_{\text {osd }} / 4=1 / 2 \sqrt{\Omega_{R} E_{G} / \hbar},
$$

where $\Omega_{R}=\sqrt{\left|\Omega_{1}\right|^{2}+\Delta^{2}}$ is the generalized Rabi frequency. If the interaction time is longer than this, Eq. (7) is no longer valid, and population transfers to states with the largest momenta (large $N$ ) are suppressed (Raman and

\footnotetext{
${ }^{6}$ The original proposal by Kapitza and Dirac (1933) was for Bragg reflection of electrons by a standing wave of light (Batelaan, 2000; Freimund et al., 2001) However, Kapitza-Dirac scattering is now most commonly used to describe diffraction of atoms by a thin grating of light.
} 

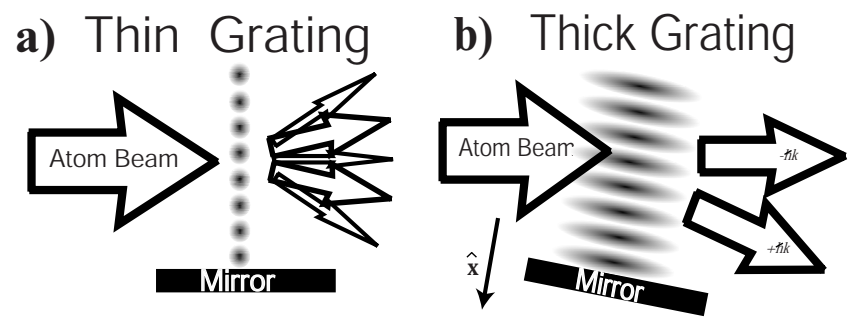

Kapitza-Dirac Diffraction
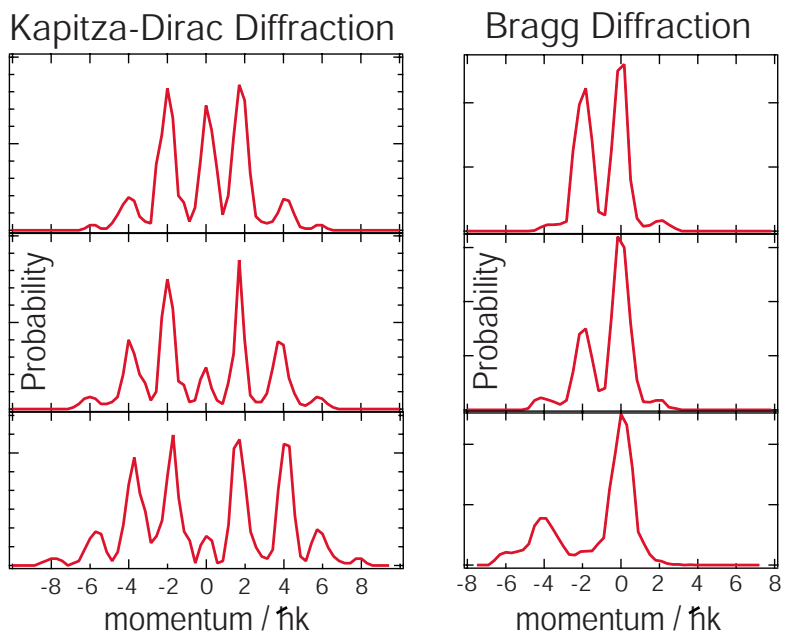

FIG. 9. (Color online) Comparison between diffraction from a thick and a thin grating. (a) Kapitza-Dirac (KD) diffraction, discussed in Sec. II.C.1. (b) Bragg diffraction, discussed in Sec. II.C.3. The top row shows the essential difference: thick vs thin gratings. The bottom row shows data obtained by the Pritchard group for KD and Bragg diffraction. Adapted from Gould et al., 1986 and Martin et al., 1988.

Nath, 1935; Moharam and Young, 1978; Wilkens et al., 1991; Keller et al., 1999; Meystre, 2001).

Early attempts to observe the Kapitza-Dirac (KD) effect with electrons were controversial [see discussion by Batelaan (2000) and Freimund et al. (2001)], and attempts with atoms were unable to eliminate the effects of spontaneous emission (Arimondo et al., 1979). ${ }^{7}$ The first observation of Kapitza-Dirac scattering by Moskowitz et al. (1983) and Gould et al. (1986) was therefore a breakthrough: it showed a symmetric double maximum and also revealed that momentum transfer was quantized in units of $2 \hbar k_{\text {light }}$ thereby indicating a coherent process. Moreover, these experiments showed that quantized momentum transfer (i.e., coherent diffraction) is possible even if the interaction time $\tau$ is much larger than the atoms' radiative lifetime, provided that the radiation is detuned from resonance.

With a BEC Kapitza-Dirac scattering was first observed at NIST by Ovchinnikov et al. (1999) and has subsequently become an everyday tool for manipulating BECs. More recently, a series of light pulses separated in

\footnotetext{
${ }^{7}$ In fact, T. Oka made a wager with D.E.P. that the MIT experiments would continue to show a maximum at zero deflection, rather than revealing two maxima displaced from the center as predicted.
}

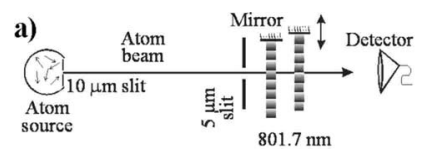

b)
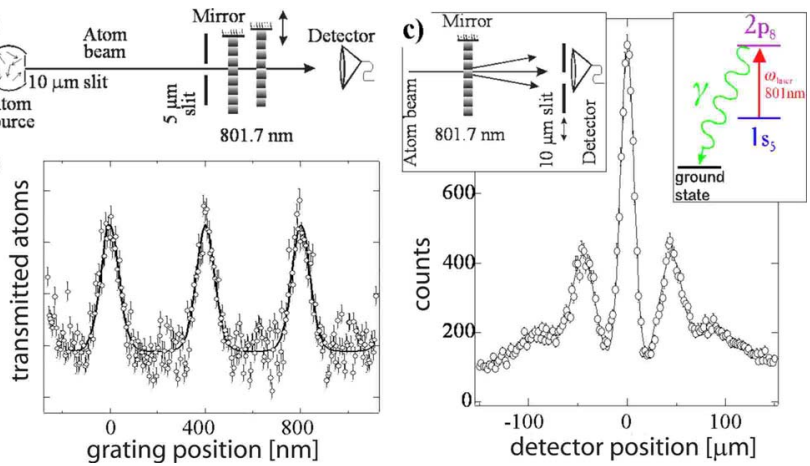

FIG. 10. (Color online) Diffraction from a measurementinduced grating. (a) Schematic of two on-resonant standing waves of light. The first causes atom diffraction. The second can be translated to analyze near-field atomic flux. (b) Periodic structure in the transmitted atomic beam. (c) Far-field atom diffraction from a measurement induced grating. Adapted from Abfalterer et al., 1997.

time [by about one-eighth of the Talbot time $\left(\tau_{T}\right.$ $\left.\left.=2 d^{2} m / h\right)\right]$ have been used to diffract atoms with high efficiency into only the \pm 1 orders (Wang et al., 2005; Wu, Wang, et al., 2005).

\section{Diffraction with on-resonant light}

Tuning the light frequency of a standing light wave to resonance with an atomic transition $(\Delta=0)$ can make an "absorptive" grating with light. This is possible when the spontaneous decay of the excited state proceeds mainly to an internal state which is not detected. (If the excited state decays back to the ground state, this process produces decoherence and diffusion in momentum space.) For a thin standing wave the atomic transmission is given by

$$
T(x)=\exp \{-(\kappa / 2)[1+\cos (G x)]\},
$$

where the absorption depth for atoms passing through the antinodes is $\kappa$. For sufficiently large absorption only atoms passing near the intensity nodes survive in their original state and the atom density evolves into a comb of narrow peaks. Since the absorption involves spontaneous emission such light structures have been called measurement induced gratings. As with all thin gratings, the diffraction pattern is then given by the scaled Fourier transform of the transmission function.

Such gratings have been used for a series of near-field (atom lithography; Talbot effect) and far-field (diffraction; interferometry) experiments, and an example is shown in Fig. 10 (Rasel et al., 1995; Johnson et al., 1996, 1998; Abfalterer et al., 1997; Jurgens et al., 2004). These experiments demonstrate that transmission of atoms through the nodes of the absorptive light masks is a coherent process.

\section{Thick gratings: Bragg diffraction}

If the standing wave is thick, one must consider the full propagation of the matter wave inside the periodic 


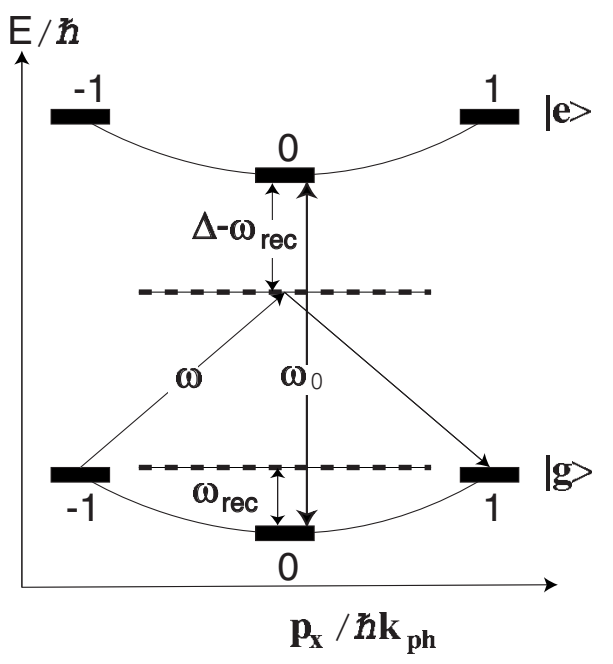

FIG. 11. Energy states on the energy-momentum dispersion curve associated with Bragg diffraction. Versions of this classic figure are found in Martin et al. (1988); Giltner et al. (1995a); Bordé (1997); Kozuma, Deng, et al. (1999); Gupta, Leanhardt, et al. (2001).

potential. The physics is characterized by multi wave (beam) interference. For two limiting cases one can regain simple models. For weak potentials, Bragg scattering; and for strong potentials, coherent channeling.

When an atomic matter wave impinges on a thick but weak light crystal, diffraction occurs only at specific angles, the Bragg angles $\theta_{B}$ defined by the Bragg condition

$$
N \lambda_{\mathrm{dB}}=\lambda_{\mathrm{ph}} \sin \left(\theta_{B}\right) .
$$

Bragg scattering, as shown in Fig. 9 (right column), transfers atoms with momentum $-p_{x}$ into a state with a single new momentum, $p_{x}=-p_{x}+\hbar G$. Momentum states in this case are defined in the frame of the standing wave in direct analogy to electron or neutron scattering from perfect crystals. Bragg scattering was first observed at MIT (Martin et al., 1988) and first observed with atoms in a Bose-Einstein condensate at NIST (Kozuma, Deng, et al., 1999). Higher-order Bragg pulses transfer multiples of $N \hbar G$ of momentum, and this has been demonstrated up to 8th order with an atomic beam (Giltner et al., 1995b; Koolen et al., 2002). Reviews of Bragg scattering appear in Durr and Rempe (1999); Obrethaler et al. (1999); Bernet et al. (2000); Gupta, Leanhardt, et al. (2001).

The Bragg scattering process can be understood in terms of absorption followed by stimulated emission (Fig. 11). Viewing Bragg scattering as a two-photon transition from the initial ground state with momentum to a final ground state (with new momentum) illuminates the close connection with Raman transitions (Gupta, Leanhardt, et al., 2001).

As a result of the coherently driven two-photon transition, the probability amplitude oscillates between the two momentum states $\left|g,-\hbar k_{\mathrm{ph}}\right\rangle$ and $\left|g,+\hbar k_{\mathrm{ph}}\right\rangle$ in a manner analogous to the Rabi oscillation of atomic popula- tion between two resonantly coupled states. The probability for Bragg scattering of atoms from off-resonant standing waves of light is

$$
P_{N=1}^{\mathrm{Bragg}}(\tau)=\sin ^{2}\left(\Omega_{1}^{2} \tau / 4 \Delta\right) .
$$

The oscillation between the two Bragg-coupled states $(N=0$ and $N=1)$ is known as the Pendellösung and has been observed for atoms (Martin et al., 1988; Oberthaler et al., 1999; Koolen et al., 2002), neutrons (Shull, 1968), electrons, and $\mathrm{x}$ rays. The nice feature with atoms is that the strength of the grating can be controlled by the intensity of the light.

The probability for $N$ th-order Bragg diffraction is

$$
P_{N}^{\mathrm{Bragg}}(\tau)=\sin ^{2}\left\{\Omega_{1}^{2 N} \tau / 2^{4 N-3}[(N-1) !]^{2} \Delta^{N} \omega_{\text {rec }}^{N-1}\right\},
$$

where we have assumed $\Delta \gg N^{2} \omega_{\text {rec }}$

Bragg diffraction of atoms from off-resonant standing waves of light is often used for studying a BECs velocity distribution because of the velocity selectivity of the Bragg condition (Kozuma, Deng, et al., 1999; Stenger et al., 1999; Blakie and Ballagh, 2000; Carusotto et al., 2000; Stamper-Kurn et al., 2001). $\sigma_{v}$ is improved by increasing the duration of interaction with the grating, as can be deduced from the time-energy uncertainty principle, $\sigma_{v}=2 / \tau G{ }^{8}$ For first-order Bragg diffraction, the minimum interaction time required to suppress all but one diffraction order is $\tau>h / E_{G} \approx 10 \mu \mathrm{s}$; so to observe Bragg scattering with a $1000 \mathrm{~m} / \mathrm{s} \mathrm{Na}$ atom beam typically requires standing waves nearly $1 \mathrm{~cm}$ thick. However, $\tau$ can be substantially increased with cold atoms, and $\sigma_{v}$ less than $1 / 30$ of the recoil velocity has been observed. For higher-order Bragg diffraction the interaction time must be $\tau \gg \pi /\left[2(N-1) \omega_{\text {rec }}\right]$.

Bragg scattering can be described as a multibeam interference as treated in the dynamical diffraction theory developed for neutron scattering. Inside the crystal one has two waves, the refracted incident "forward" wave $\left(k_{F}\right)$ and the diffracted "Bragg" wave $\left(k_{B}\right)$. These form a standing atomic wave field, and the diffraction condition $\left(k_{B}-k_{F}=G\right)$ implies that the standing atomic wave has the same periodicity as the standing light wave. At any location inside the lattice, the exact location of atomic probability density depends on $k_{F}, k_{B}$ and the phase difference between these two waves.

For incidence exactly on the Bragg condition the nodal planes of the two wave fields are parallel to the lattice planes. The eigenstates of the atomic wave field in the light crystal are two Bloch states, one exhibiting maximal $\left(\Psi_{\max }\right)$ and the other minimal $\left(\Psi_{\min }\right)$ interaction:

$$
\Psi_{\max }=\frac{1}{2}\left[e^{i G x / 2}+e^{-i G x / 2}\right]=\cos (G x / 2),
$$

\footnotetext{
${ }^{8}$ States lie on the energy-momentum dispersion curve $(E$ $=p^{2} / 2 m$ ) with quantized momentum. Finite interaction times $(\tau)$ allow states to be populated with a range of energy $\sigma_{E}$ $=p \sigma_{v}=2 \hbar / \tau$. For an $N$ th-order Bragg process, the state momentum is centered around $p=N \hbar G$. Hence $\sigma_{v}=2 / N \tau G$.
} 


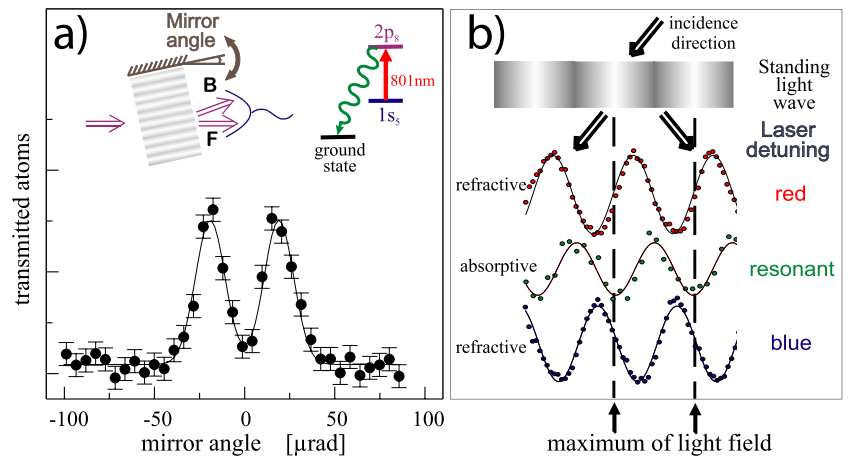

FIG. 12. (Color online) Bragg diffraction of atoms from resonant standing waves of light. (a) Atoms entering the light crystal at the Bragg angle are less likely to emit a spontaneous photon and therefore survive the on resonant light field (anomalous transmission). (b) A resonant standing wave inside a light crystal serves to measure the atom wave fields inside the crystal. For on-resonance light crystals one observes the minimal coupled Bloch state. Adapted from Oberthaler, Abfalterer, et al., 1996.

$$
\Psi_{\min }=\frac{1}{2}\left[e^{i G x / 2}-e^{-i G x / 2}\right]=i \sin (G x / 2)
$$

For $\Psi_{\max }$ the antinodes of the atomic wave field coincide with the planes of maximal light intensity, and for $\Psi_{\text {min }}$ the antinodes of atomic wave fields are at the nodes of the standing light wave. These states are very closely related to the coupled and noncoupled states in velocity selective coherent population trapping (Aspect et al., 1988).

The total wave function is that superposition of $\Psi_{\max }$ and $\Psi_{\min }$ which satisfies the initial boundary condition. The incoming wave is projected onto the two Bloch states which propagate through the crystal accumulating a relative phase shift. At the exit, the final populations in the two beams is determined by interference and depends on this relative phase [following Eq. (11)].

Bragg scattering can also be observed with absorptive, on-resonant light structures (Oberthaler, Abfalterer, et al., 1996) and combinations of both on- and off-resonant light fields (Keller et al., 1997). One remarkable phenomenon is that the total number of atoms transmitted through a weak on-resonant standing light wave increases if the incident angle fulfills the Bragg condition, as shown in Fig. 12. This observation is similar to what Borrmann (1941) discovered for $\mathrm{x}$ rays and called anomalous transmission.

The observed anomalous transmission effect can be understood in the framework of the two beam approximation outlined above. The rate of de-population of the atomic state is proportional to the light intensity seen by the atoms and therefore to the overlap between the atom wave field and the standing light field. The minimally coupled state $\Psi_{\min }$ will propagate much further into the crystal than $\Psi_{\text {max }}$. At the exit phase the propagating wave field will be nearly pure $\Psi_{\min }$. As a consequence one sees two output beams of (nearly) equal intensity.
Inserting an absorptive mask (Abfalterer et al., 1997) inside the light crystal allows one to observe the standing matter wave pattern inside the crystal (Oberthaler, Abfalterer, et al., 1996, 1999) and verify the relative positions between the light field and $\Psi_{\min }$.

Indeed, tailored complex potentials for atoms can be made out of combinations of bi-chromatic standing waves (Keller et al., 1997). For example, a superposition of standing waves (one on- and one off-resonance) with a phase shift $\Delta \varphi= \pm \pi / 2$ results in a combined potential of $U(x)=U_{0} e^{ \pm i G x}$ which, in contrast to a standing wave, has only one momentum component. Such a potential can therefore only diffract in one direction. As this predicts, various diffraction orders can be suppressed by adjusting the phase difference between the absorptive and the refractive grating. The lack of symmetry is referred to as a violation of Friedel's law. The asymmetry in the observed patterns can also be understood as an interference effect between diffraction at refractive and absorptive "subcrystals" spatially displaced with respect to each other (Keller et al., 1997).

\section{Bloch oscillations}

Bloch oscillations were predicted by Bloch (1929) and Zener (1934) as an interference phenomenon in connection with the electronic transport in crystal lattices, but can in general also be observed in any system where accelerated matter waves move through a periodic potential. In a simple physical picture the Bloch oscillations can be viewed as repeated Bragg reflection from an accelerating grating. To observe high-contrast Bloch oscillations it is desirable to prepare the initial sample well localized in momentum space, with a width of the momentum distribution much smaller than the Brilloiuin zone. Therefore a BEC would be an ideal starting condition.

The first to observe Bloch oscillations with atomic matter waves was Dahan et al. (1996), who studied the motion of thermal atoms in an accelerated lattice. Since then, because optical lattices can be precisely controlled, Bloch oscillations have been used for precision measurements of quantities related to acceleration such as $g$ or $\hbar / m_{\text {atom }}$.

In a real experiments atom-atom interactions damp the Bloch oscillations (by de-phasing). Roati et al. (2004) showed that Bloch oscillations survive much longer for noninteracting fermions $\left({ }^{40} \mathrm{~K}\right)$ when compared with bosons $\left({ }^{87} \mathrm{Rb}\right)$. Very long lasting Bloch oscillations were observed for weakly interacting bosons $\left({ }^{88} \mathrm{Sr}\right)$ by Ferrari et al. (2006) with interactions switched off by tuning with a Feshbach resonance in ${ }^{133} \mathrm{Cs}$ by Gustavsson et al. (2007) or ${ }^{39} \mathrm{~K}$ by Fattori, D'Errico, et al. (2008) (see Fig. 13). Bloch oscillations with interactions minimized by Feshbach resonances were used by Fattori, Roati, et al. (2008) to study the small remaining magnetic dipolar interactions in a BEC. 

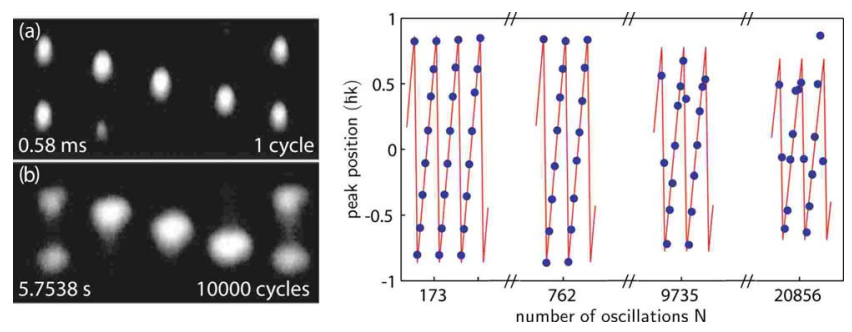

FIG. 13. (Color online) Observation of long lasting Bloch oscillations in Cs. The images (left) and graph (right) show how atoms released from a vertically oriented lattice have a velocity that oscillates as a function of hold time. To suppress damping, atom-atom interactions were switched off by tuning the scattering length close to zero by applying a magnetic field of 17.12 G. Adapted from Gustavsson et al., 2007.

\section{Coherent channeling}

When the lattice potential becomes higher then $E_{G}$ [Eq. (6)] the atoms can be localized in the standing light wave. Atoms impinging on such a strong light crystal are then guided in the troughs through the crystal, and can interfere afterwards. Such guiding is called channeling. Channeling of electron beams (Joy et al., 1982) and ion beams (Feldman et al., 1982) in material crystals is related to channeling of atoms in optical lattices (Salomon et al., 1987; Horne et al., 1999; Keller et al., 1999). If the process is coherent, one can observe a diffraction pattern reminiscent of the KD diffraction from a thin grating; see Fig. 14.

\section{The Talbot effect}

We now turn from far-field atom diffraction to the near-field region, where a host of different interference effects occur. The well-known optical self-imaging of a grating discovered by Talbot in 1832 is most important. It has many applications in image processing and synthesis, photolithography, optical testing, and optical metrology (Patorski, 1989), and has proven to be a powerful

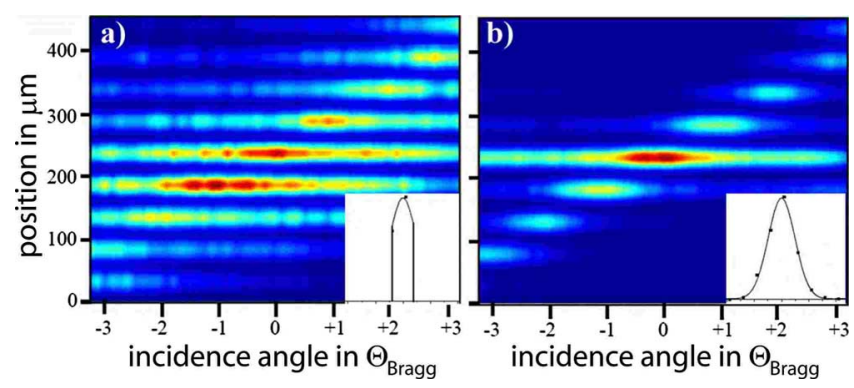

FIG. 14. (Color online) Coherent channeling of atoms through a strong light crystal. (a) When the light crystal turns on abruptly (see inset) many transverse momentum states are populated, and a large number of outgoing diffraction orders are observed. (b) Atoms entering the light crystal slowly (adiabatically) only occupy the lowest energy states, hence only one or two output beams are observed, as in Bragg scattering. From Keller et al., 1999.
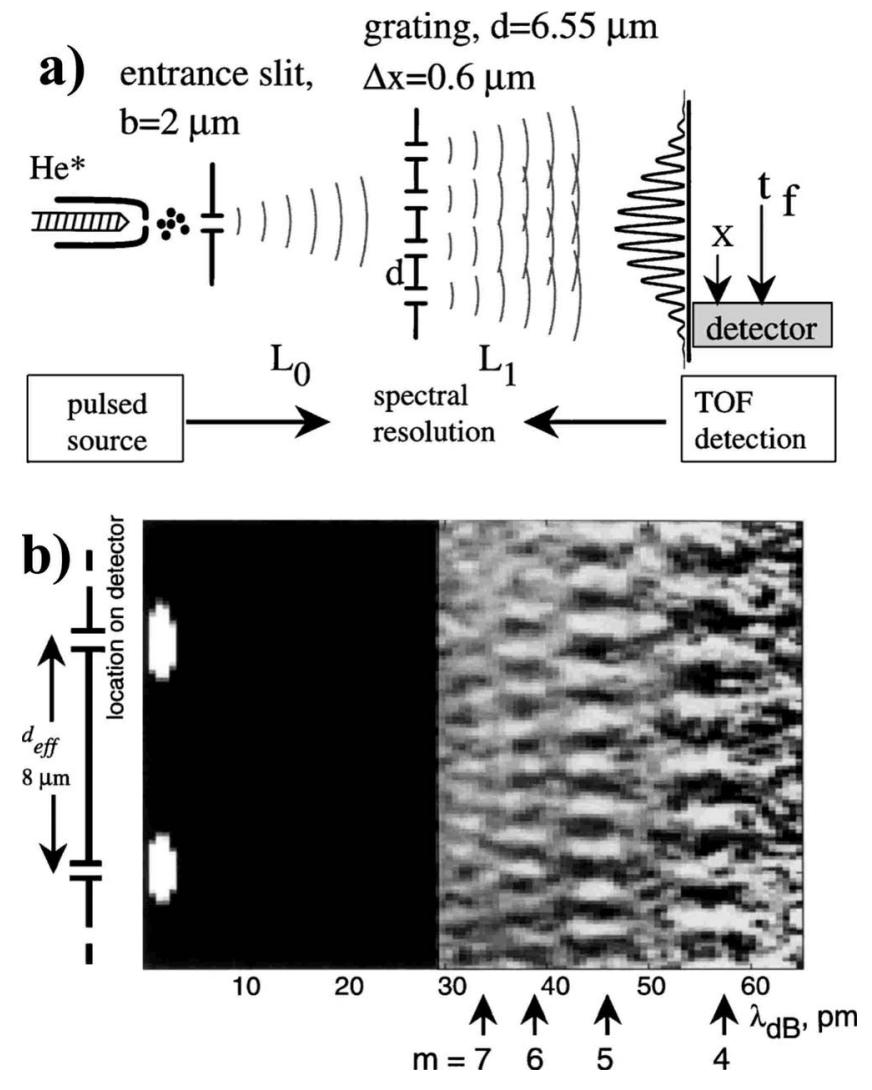

FIG. 15. The Talbot effect. (a) Schematic of a pulsed source and a time-resolved detector used to observe near-field diffraction from a nanograting with 0.6 - $\mu$ m-diameter windows spaced with a period of $6.55 \mu \mathrm{m}$. (b) Higher-order Talbot fringes. The spatial atom distribution vs de Broglie wavelength is plotted. The arrows indicate locations at which Talbot fringes of the $m$ th order are observed. Adapted from Nowak et al., 1997.

tool for interference experiments with matter waves.

Plane waves incident on a periodic structure form a "self-image" of the structure at the Talbot distance $L_{T}$ $=2 d^{2} / \lambda_{\mathrm{dB}}$ and again at integer multiples of the Talbot length. At half the Talbot distance a similar self-image is formed but displaced by half a period. At certain intermediate distances higher-order Talbot images are formed. These have a spatial frequency that is higher than the original grating by a ratio of small integers. The position and contrast of the subperiod images are determined by Fresnel diffraction as discussed by Patorski (1989); Clauser and Reinisch (1992); Clauser and Li (1994b). The replica (Fresnel) images and higher-order (Fourier) images are used in a Talbot-Lau interferometer (Brezger et al., 2003).

Talbot fringes were first observed with an atom beam and nanostructure gratings by Schmiedmayer et al. (1993); Clauser and Li (1994b); Chapman, Ekstrom, Hammond, Schmiedmayer, et al. (1995) and higher-order Talbot fringes were observed by Nowak et al. (1997); see Fig. 15. The Talbot effect has also been studied with on-resonant light (Turlapov et al., 2003, 2005), and Talbot revivals have been observed in the time evolution of atom clouds after pulses of off-resonant standing waves 
of light (Cahn et al., 1997; Deng et al., 1999). The Talbot time is $\tau_{T}=L_{T} / v=2 d^{2} \mathrm{~m} / \mathrm{h}$.

Rohwedder (2001) proposed detecting the Talbot effect for atoms trapped in wave guides, and Ruostekoski et al. (2001) discussed the formation of vortices in BEC as a result of the Talbot effect. Proposals to use the Talbot effect to study the state of electromagnetic fields in cavities have been discussed by Rohwedder et al. (1999) and Rohwedder and Santos (2000). Using the Talbot effect with multiple phase gratings has been proposed as a way to make more efficient beam splitters for atom waves (Rohwedder, 1999, 2000), and this is related to the standing-wave light-pulse sequence described by $\mathrm{Wu}$, Wang, et al. (2005).

The Lau effect is a closely related phenomenon in which incoherent light incident on two gratings causes fringes on a distant screen, provided that the gratings are separated by a half-integer multiple of the Talbot length. References for the Lau effect in light optics include Lau (1948); Bartelt and Jahns (1979); Jahns and Lohmann (1979); Patorski (1989). In essence, for the Lau effect the first grating serves as an array of mutually incoherent sources and Fresnel diffraction from the second grating makes the pattern on the screen. This forms the basis for Talbot-Lau interferometers which we discuss in Sec. III.

A promising application of Talbot (or Lau) imaging with atoms is atom lithography as demonstrated by Timp et al. (1992), and McClelland et al. (2004), and many others. For reviews, see Bell et al. (1999) and Meschede and Metcalf (2003). It is possible to write smaller gratings and features using the reduced period intermediate images discussed above. Similar Fourier images have been used for $\mathrm{x}$ rays to write half-period gratings (Flanders et al., 1979) and to construct x-ray interferometers (David et al., 2002; Momose et al., 2003; Weitkamp et al., 2005). Grating self-images may also be used in quantum optics experiments to produce a periodic atom density in an optical resonator.

\section{E. Time-dependent diffraction}

Many new interference effects arise when the diffracting structures are modulated in time, a situation we have not considered previously (except for revivals at the Talbot time after pulsed gratings). These new effects arise with matter waves because the vacuum is dispersive for atoms-particles with shorter wavelength (higher energy) propagate faster than those with longer wavelengths. In contrast, for light in vacuum all wavelengths propagate at a constant speed $c$.

Two matter wave components interfering at $(x, t)$ may have propagated from the same $x^{\prime}$ but originated from there at different times $t^{\prime}$ (if they have different velocity). Time-dependent boundary conditions can cause matter wave diffraction phenomena in time that are similar to spatial diffraction phenomena arising from spatially dependent boundary conditions. This was first discussed by Moshinsky (1952), who argued that after opening a shutter one should observe a rise in the mat- ter wave intensity with Fresnel fringes in time, similar to the diffraction of an edge in space. He called this very general phenomena diffraction in time. Similarly, the opening and closing of a shutter results in a single slit diffraction in time; two successive openings makes a double slit; and a periodic change in the opening of the slit produces a diffraction pattern in time. With diffraction in time, new frequency (energy) components are created (as in an acoustic-optic modulator), resulting in components with new momenta. In analogy to diffraction in space one finds that diffraction in time has both near-field and far-field regimes. One also observes Raman-Nath and Bragg regimes, depending on the duration of the interaction and the amount of energy (frequency) transfer.

\section{Vibrating mirrors}

Even though diffraction in time of matter waves was predicted in 1952 the first experimental demonstrations had to wait until the late 1980s. The experimental difficulty in seeing diffraction in time is that the time scale for switching has to be faster than the inverse frequency (energy) width of the incident matter wave. This condition is the time equivalent to coherent illumination of adjacent slits in spatial diffraction. The first (explicit) experiments demonstrating diffraction in time used ultracold neutrons reflecting from vibrating mirrors (Hamilton et al., 1987; Felber et al., 1990; Hils et al., 1998). The sidebands of the momentum components were observed.

A study of diffraction and interference in time was performed by Dalibard and co-workers at the ENS in Paris using ultracold atoms reflecting from a switchable atom mirror (Steane et al., 1995; Arndt et al., 1996; Szriftgiser et al., 1996). Ultracold Cs atoms ( $T$ $\sim 3.6 \mu \mathrm{K})$ released from an optical molasses fell $3 \mathrm{~mm}$, and were reflected from an evanescent atom mirror. By pulsing the evanescent light field one can switch the mirror on and off, creating time-dependent apertures that are diffractive structures in the spirit of Moshinsky (1952).

Even for these ultracold Cs atoms the energy spread ( $7 \mathrm{MHz}$ ) is too large for the time-diffraction experiment, so a very narrow energy window was selected by two $(0.4 \mathrm{~ms})$ temporal slits. The first slit was positioned $26 \mathrm{~ms}$ after the atoms were released. Switching on the mirror a second time, $52 \mathrm{~ms}$ later, selected a very narrow energy slice in the same way as a two-slit collimation selects a very narrow transverse velocity slice. The arrival time of the atoms at the final "screen" was measured by fluorescence induced by a light sheet.

If the second slit is very narrow $(<10 \mu \mathrm{s})$, one observes single-slit diffraction in time; if the mirror is pulsed on twice within the coherence time of the atomic ensemble, one observes double-slit interference in time; and many pulses lead to a time-dependent flux analogous to a grating diffraction pattern, as shown in Fig. 16. From the measurement of the arrival times the energy distribution can be reconstructed. Similar diffraction in 

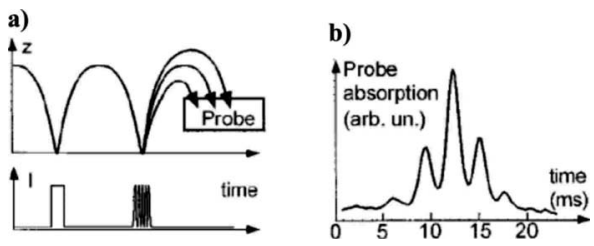

FIG. 16. Diffraction in time from a pulsed mirror. (a) Schematic of the experiment, showing atom trajectories and a trace indicating when the mirror was switched on. The first pulse acts as a slit in time, the second pulse is modulated so that it acts as a grating in time. (b) The diffraction pattern in time manifests as different energy components in the resulting atomic beam. Adapted from Steane et al., 1995, and CohenTannoudji, 1998.

time is observed when a BEC is reflected from a vibrating mirror (Colombe et al., 2005).

Because the interaction time between the atoms and the mirror potential $(<1 \mu \mathrm{s})$ was always much smaller than the modulation time scale $(>10 \mu \mathrm{s})$, these experiments are in the "thin grating" (Raman-Nath) regime for diffraction in time.

\section{Oscillating potentials}

When matter waves traverse a time-modulated potential one can observe coherent exchange of energy between the oscillating field and the matter wave. This was demonstrated in a neutron interference experiment (Summhammer et al., 1995) where an oscillating magnetic potential was applied to one path of a neutron interferometer. Coherent exchange of up to five quanta $\hbar \omega$ was observed in the interference patterns, even though the transit time through the oscillating potential was much shorter than the oscillation period.

\section{Modulated light crystals}

The time equivalent of spatial Bragg scattering can be reached if the interaction time between the atoms and the potential is long enough to accommodate many cycles of modulation. When a light crystal is modulated much faster than the transit time, momentum is transferred in reciprocal lattice vector units and energy in sidebands at the modulation frequency. This leads to Bragg diffraction at two new incident angles.

Bragg scattering in time can be understood as a transition between two energy and momentum states. The intensity modulation frequency of the standing light wave compensates the detuning of the Bragg angle. The frequency of the de Broglie wave diffracted at the new Bragg angles is shifted by $\pm \hbar \omega_{\text {mod }}$ (Bernet et al., 1996, 2000). Thus an amplitude modulated light crystal realizes a coherent frequency shifter for a continuous atomic beam. It acts on matter waves just as an acousto-optic modulator acts on photons, shifting the frequency (kinetic energy) and requiring an accompanying momentum (direction) change.

In a complementary point of view, the new Bragg angles can be understood from looking at the light crys-
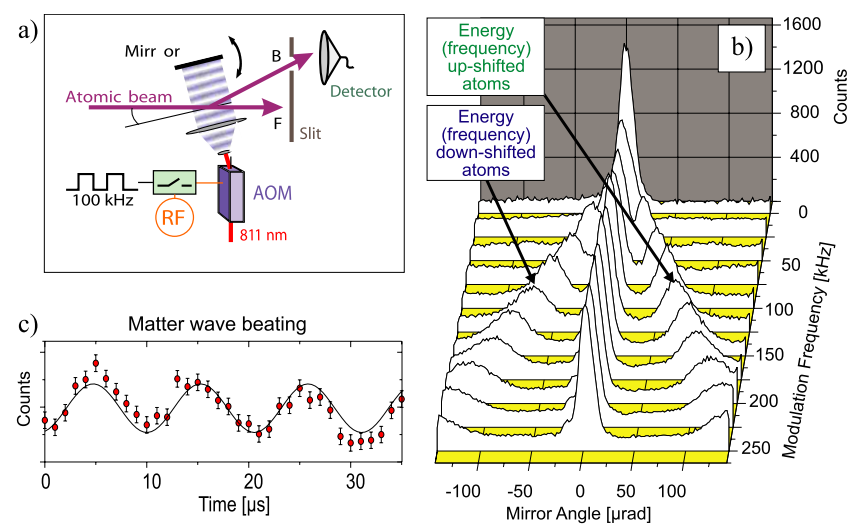

FIG. 17. (Color online) Frequency shifter for matter waves. (a) A time-modulated light crystal causes diffraction in time and space. (b) Rocking curves show how the Bragg angle for frequency-shifted matter waves is controlled by the grating modulation frequency. (c) Beating between frequency shifted and unshifted matter waves. Adapted from Bernet et al., 1996.

tal itself. The modulation creates sidebands $\pm \omega_{\bmod }$ on the laser light, creating moving crystals which come from the interference between the carrier and sidebands. Bragg diffraction from the moving crystals occurs where the Bragg condition is fulfilled in the frame co-moving with the crystal, resulting in diffraction of the incident beam to new incident angles.

The coherent frequency shift of the Bragg-diffracted atoms can be measured by interferometric superposition with the transmitted beam. Directly behind the light crystal the two outgoing beams form an atomic interference pattern which can be probed by a thin absorptive light grating (Abfalterer et al., 1997). Since the energy of the diffracted atoms is shifted by $\hbar \omega_{\text {mod }}$, the atomic interference pattern continuously moves; this results in a temporally oscillating atomic transmission through the absorption grating (Fig. 17).

Starting from this basic principle of frequency shifting by diffraction from a time-dependent light crystal many other time-dependent interference phenomena were studied for matter waves (Bernet et al., 1999, 2000). Light crystals are an ideal tool for these experiments since one can easily tailor potentials by controlling the laser intensity and frequency and create more complex structures by superimposing different independently controlled crystals.

For example, using light from two different lasers one can create two coinciding light crystals generated in front of the retroreflection mirror. Varying detuning and phase between the two modulated crystals creates situations where diffraction is completely suppressed, or where either the frequency unshifted or the frequency shifted order is suppressed (Fig. 18). The combination of real and imaginary potentials can produce a driving potential of the form $U(t) \sim e^{ \pm i \omega_{m} t}$ which contains only positive (negative) frequency components, respectively. Such a modulation can only drive transitions up in energy (or down in energy). 


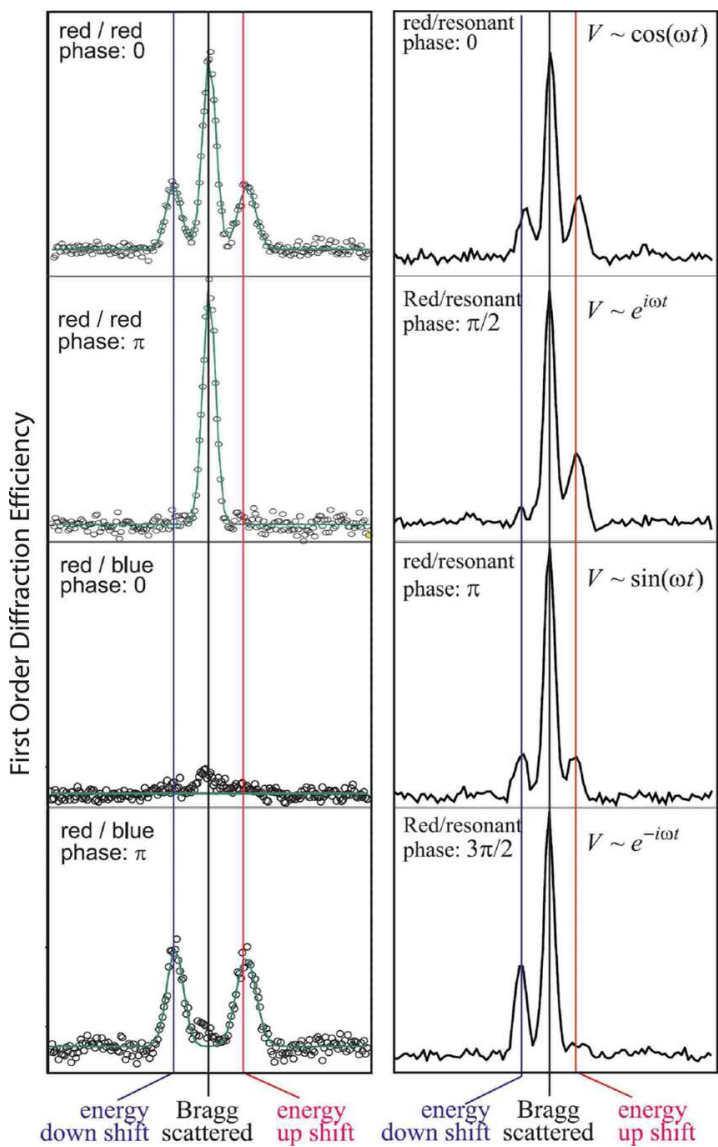

FIG. 18. (Color online) Diffraction in time from two superimposed light crystals with a controlled relative phase between the modulations. The first-order diffraction efficiency is plotted as a function of grating angle (to generate a "rocking curve") for a variety of gratings. Left: Two off-resonant light crystals are superimposed. The relative phase of the temporal modulation controls the intensity of the frequency shifted and unshifted Bragg beams. Right: An on-resonant and an offresonant crystal are superimposed. The relative phase controls the time-dependent potential. For phase $\pi / 3(3 \pi / 2)$ only frequency up (down) shifted components appear. Adapted from Bernet et al., 2000.

\section{F. Summary of diffractive Atom Optics}

To summarize, in Sec. II we reviewed atom diffraction from nanostructures and standing waves of light. Nanostructures absorb atoms, can be arbitrarily patterned (e.g., holograms), and affect all atomic and molecular species. Standing waves of light can make a phase (or in some cases amplitude) grating for a particular species of atom in a specific state. Light gratings can be thick or thin, strong or weak, and can be modulated in time. Both types of grating exhibit interesting and useful interference phenomena in both the near- and far-field regimes.

Figure 19 summarizes the diffraction efficiency of four different kinds of time-independent gratings: two absorbing gratings (nanostructures and standing waves of on-resonant light) and two nondissipative gratings (in the Kapitza-Dirac and Bragg regimes). These efficien-
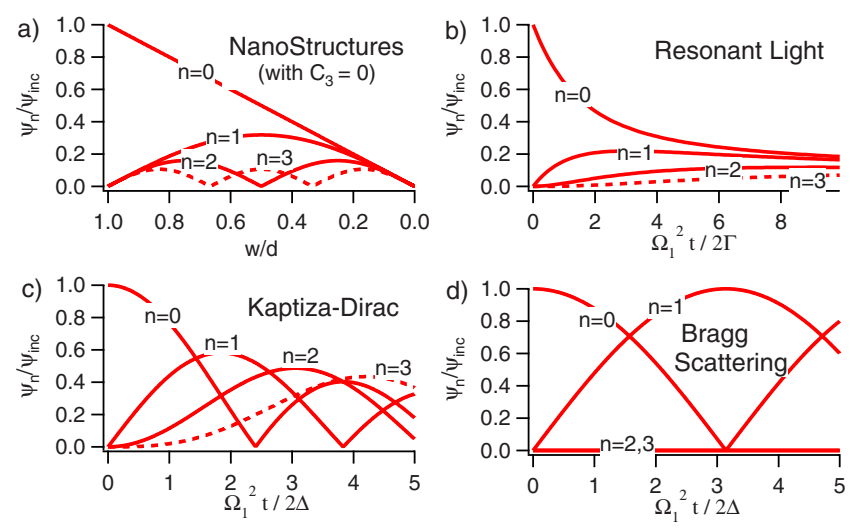

FIG. 19. (Color online) Summary of diffraction efficiency for different types of gratings. The atomic de Broglie wave amplitude in the $n$th diffraction order (normalized to the incident wave amplitude) is plotted for (a) nanostructures using Eq. (3), (b) standing waves of on-resonant light using Eqs. (4) and (9), (c) Kapitza-Dirac (thin phase mask) diffraction using Eq. (7), and (d) Bragg (thick crystal) scattering using Eq. (11).

cies are given by Eqs. (3), (7), and (11), and the Fourier transform of Eq. (9).

Figure 20 summarizes thick and thin gratings in space and also in time with Ewald constructions to denote energy and momentum of the diffracted and incident atom waves. The diffraction from (modulated) standing waves of light can also be summarized with the Bloch band spectroscopy picture (Bernet et al., 2000; Champenois et al., 2001a).

\section{G. Other coherent beam splitters}

Whereas diffraction occurs without changing the atom's internal state, another important class of beam splitters uses laser or rf transitions that do change atoms' internal state while transferring momentum. Therefore as they coherently split atomic wave functions into two (or more) pieces they cause entanglement between the a) Bragg
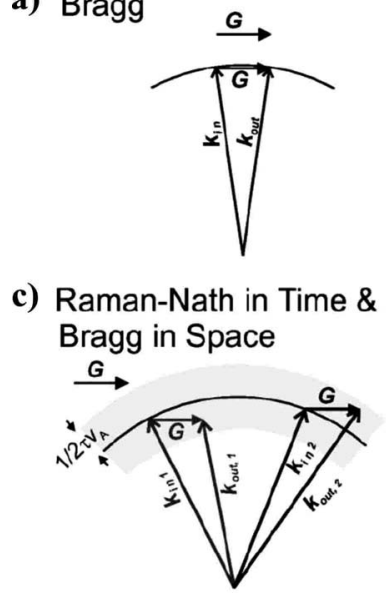

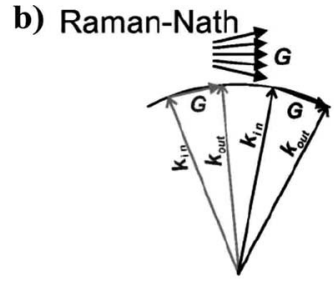

d) Bragg in Space \& Time

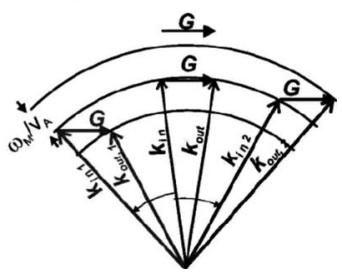

FIG. 20. Momentum diagrams for (a) a thick grating, (b) a thin grating, (c) a thick pulsed grating, and (d) a thick harmonically modulated grating. From Bernet et al., 2000. 
atomic motion and the internal atomic states. Important examples that are used in atom interferometry include absorption from a traveling wave of light (Bordé, 1989, 1997), stimulated Raman transitions (Kasevich and Chu, 1992), and longitudinal rf spectroscopy (Gupta, Kokorowski, et al., 2001). The longitudinal Stern-Gerlach effect (Miniatura et al., 1991) also causes entanglement between motion and internal degrees of freedom. We discuss these in Sec. III on atom interferometry.

Potentials that change slowly from single well to double well represent an entirely new type of beam splitter that is more applicable to trapped atoms than propagating light. We discuss this tool for coherent splitting of atomic wave functions in the next section on atom interferometry.

Reflecting surfaces have been used for atom diffraction, atom holography, and Young's experiment with atoms; see, e.g., Deutschmann et al. (1993); Christ et al. (1994); Landragin et al. (1997); Cognet et al. (1998); Shimizu and Fujita (2002b); Kohno et al. (2003); Esteve et al. (2004); and Gunther et al. (2005, 2007), respectively. The challenges of using reflection-type atom optical elements include low reflection probability and strict requirements for flatness in order to maintain atom wave coherence. The toolkit for coherent atom optics is expanded by quantum reflection, in which atom waves reflect from an attractive potential, and also classical reflection, where repulsive potentials can be formed with evanescent waves of blue-detuned light or engineered magnetic domains. Various mirrors for atoms have been discussed by Seifert et al. (1994); Kaiser et al. (1996); Landragin et al. (1996); Henkel et al. (1997, 1999); Berkhout et al. (1999); Marani et al. (2000); Shimizu (2001); Savalli et al. (2002); Shimizu and Fujita (2002a); Fortagh and Zimmermann (2007).

\section{ATOM INTERFEROMETERS}

\section{A. Introduction}

The essential features of interferometers generally and atom interferometers in particular are listed in the succession of five steps: (i) prepare the initial state, (ii) split the wave functions coherently into two or more states, (iii) apply interactions that affect the two states differentially, generally due to their different spatial location, (iv) recombine these components coherently, and (v) measure the phase shift of the detected fringes.

The crucial step of coherent splitting (ii) has been accomplished for atom interferometers using diffraction gratings, photon absorption, Raman transitions, longitudinal Stern-Gerlach magnets, and even physical separation of confined atoms into multiple potential wells. In the following we discuss these in the framework of the interferometers in which they have been used, and review the basic features of several atom interferometer designs. A detailed survey of scientific research with atom interferometers is given in Secs. IV-VI.

\section{General design considerations}

When designing and building interferometers for atoms and molecules, one must consider key differences between matter waves and light. The dispersion relations, the coherence properties, and our tools to control the two different kinds of waves are among the important differences.

One striking difference is the fact that matter waves have short deBroglie wavelengths $(\sim 10 \mathrm{pm}$ for thermal atoms, up to $\sim 1 \mu \mathrm{m}$ for ultracold atoms), and also have a very short coherence lengths $(\sim 100 \mathrm{pm}$ for thermal atomic beams, and seldom larger than $10 \mu \mathrm{m}$ even for atom lasers or BEC). This requires that the period and the position of the interference fringes must be independent of the de Broglie wavelength of the incident atoms. In optical parlance this is a property of white light interferometers.

A second concern with atoms is that they interact strongly with each other. Therefore matter waves are often nonlinear, especially when atoms have significant density as in a BEC or atom laser.

A third distinguishing feature is that atoms can be trapped. This leads to a new class of interferometers for confined particles, which we discuss at the end of this section.

\section{White light interferometetry}

The challenge of building a white light interferometer for matter waves is most frequently met by the threegrating Mach-Zehnder (MZ) layout. This design was used for the first electron interferometer by Marton (1952), for the first neutron interferometer by Rauch et al. (1974) and for the first atom interferometer that spatially separated the atoms by Keith et al. (1991). In the $\mathrm{MZ}$ interferometer the role of splitter and recombiner is taken up by diffraction gratings. They also serve as the mirrors that redirect the separating atom waves back together. (In fact, simple mirrors will not serve this purpose if the initial state is not extremely well collimated. This is because most interferometer designs that employ simple mirrors will make the fringe phase strongly correlated with input beam position and direction.) Simpson (1954) noted that with grating interferometers, "the fringe spacing is independent of wavelength. This 'achromatic' behavior... appears to be characteristic of instruments using diffraction for beam splitting."

The explanation is that diffraction separates the split states by the lattice momentum, then reverses this momentum difference prior to recombination. Faster atoms will diffract to smaller angles resulting in less transverse separation downstream, but will produce the same size fringes upon recombining with their smaller angle due to their shorter de Broglie wavelength. For three evenly spaced gratings, the fringe phase is independent of incident wavelength, surprisingly also for asymmetric designs [such as that in Fig. 21(a)] where the intensity 
a)

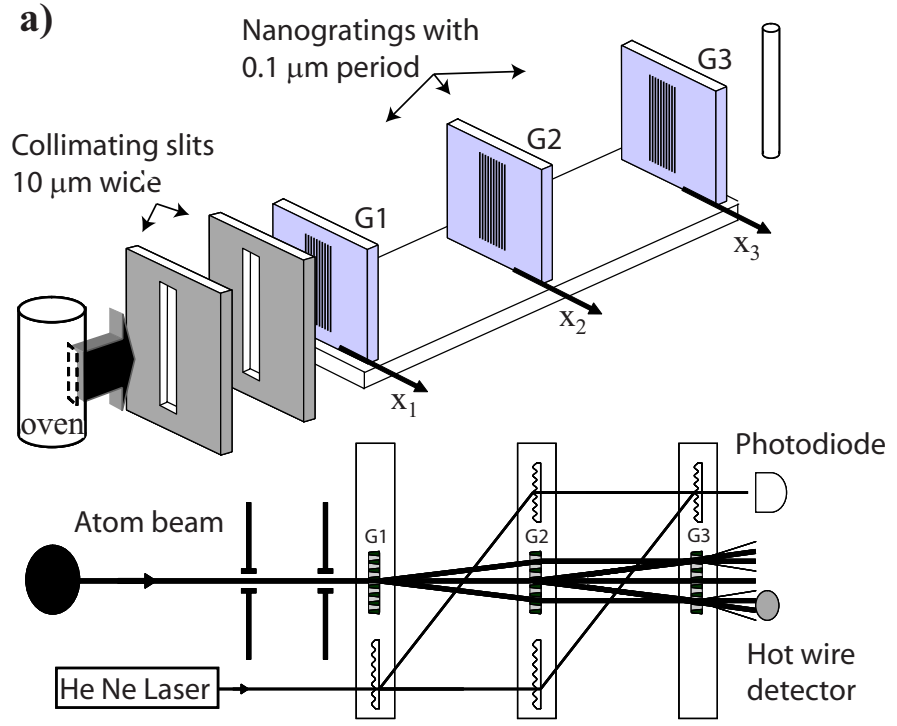

b)

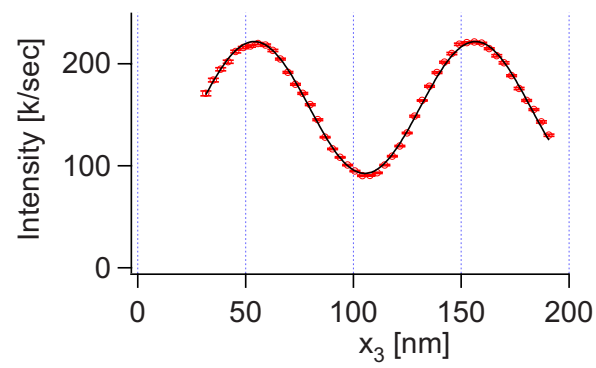

c)

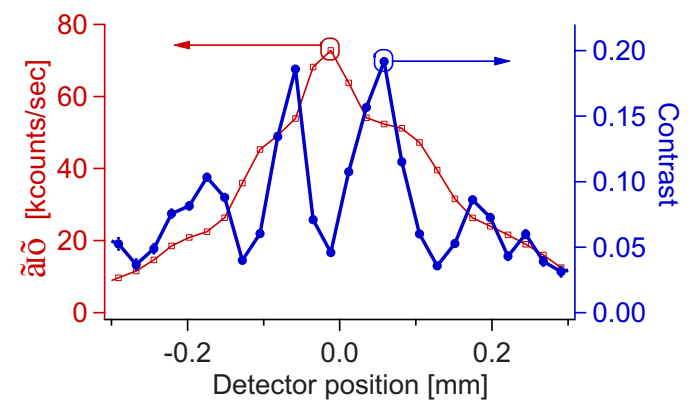

FIG. 21. (Color online) Three grating Mach-Zehnder atom interferometers. (a) Atom interferometer setup used in Keith et al. (1991). (b) Interference fringe data and best fit with $\langle I\rangle=157000$ counts per second and $C=0.42$. A total of $5 \mathrm{~s}$ of data are shown and the uncertainty in phase calculated by Eq. (19) is $\sigma_{\phi}=2.7 \times 10^{-3} \mathrm{rad}$. (c) Average intensity $\langle I\rangle$ and contrast $C$ as a function of detector position [under different conditions than (b)].

maximum for different wavelengths occurs at different distances off the symmetry axis.

Many diffraction-based interferometers produce fringes when illuminated with a source whose transverse coherence length is much less than its (large) physical width, or even the grating period. Under such conditions, the different diffraction orders will not be separated, so diffraction cannot be resolved and it will not be possible to exploit the physical separation of the orders to apply an interaction to only one arm of the interferometer. Nevertheless, high-contrast fringes will still be formed.

The three-grating interferometer produces a "position echo" as discussed by Chebotayev et al. (1985). Starting at one grating opening, one arm evolves laterally with $\hbar G$ more momentum for some time, the momenta are reversed, and the other arm evolves with the same momentum excess for the same time, coming back together with the first arm at the third grating. If the gratings are in registry, an atom's trapezoidal pattern starts at a slot on the first grating, is centered on either a middle grating slot or groove, and recombines in a slot at the third grating. Not surprisingly, spin echo and time-domain echo techniques (discussed below) also offer possibilities for building an interferometer that works even with a distribution of incident transverse atomic momenta.

\section{Types and categories}

A large variety of atom and molecule interferometers have been built since 1991. The list includes Mach-

\footnotetext{
${ }^{9}$ The design in Fig. 21(a) is asymmetric because the interferometer paths are formed by diffraction orders 0 and 1 for one arm, and orders 1 and -1 for the other.
}

Zehnder, Talbot-Lau, optical Ramsey-Bordé, and stimulated Raman transition interferometers. There are also longitudinal Stern-Gerlach and longitudinal rf interferometers. Some of these designs render the interference fringes in position space. Some make fringes in momentum space. Still other designs make the interference fringes observable in internal atomic state space. We catalog these interferometers according to their features before examining each in detail.

(i) Internal state changing interferometers are one broad category. Some beam splitters change an atom's internal state, analogous to a polarizing beam splitter in light optics. For example, stimulated Raman transitions entangle internal and external states, so atoms in these interferometers are in a coherent superposition of different momentum-spin states.

(ii) Time domain versus space domain is another broad classification. In a time-domain interferometer, the beam splitters are pulsed so all atoms interact with the gratings and the interferometer for the same amount of time.

(iii) Near-field (Talbot-Lau) and far-field (MachZehnder) classification applies for diffractive atom optics. Near-field interferometers can function even with poorly collimated beams, but the gratings in a Talbot-Lau interferometer (TLI) must be separated by precise multiples of the Talbot length or else the contrast degrades.

(iv) Separated path interferometers are a special category of atom interferometer in which the paths are sufficiently physically separated that the 
atom wave in one arm can be isolated and interactions can be applied to it alone.

(v) Freely propagating cold atoms can have long times of flight $\left(\sim \frac{1}{2} \mathrm{~s}\right)$ as compared to thermal atom beams $(\sim 1 \mathrm{~ms})$. Confinement in a trap during the interferometer operation may soon provide even longer interaction times.

(vi) Atom traps and waveguides offer the possibility of making confined atom interferometers in which the atom wave function is split in coordinate space rather than momentum space. Obviously, the ability to interfere with atoms that are spatially confined in all three dimensions throughout the entire interferometer is unprecedented with light interferometers. Additional topologies such as multiple wells, ring traps, and longitudinal waveguides have also been demonstrated.

Finally, we distinguish single atom interferometers from those in which (nonlinear) collective effects are significant. Even atoms launched from a magneto-optical trap are generally not dense enough to cause significant nonlinear effects. Interferometry with Bose-Einstein condensates (or atom lasers), on the other hand, can show nonlinear atom optics phenomena that range in significance from phase noise to number squeezing and Josephson oscillations.

The distinctions (i) internal state changing versus state preserving, (ii) space domain versus time domain, (iii) near field versus far field, (iv) separated path or not, (v) trapped or freely propagating, (vi) dilute versus dense all affect the performance of atom interferometers for different applications.

Since the first atom interferometers were built for $\mathrm{Na}$, Cs, $\mathrm{Mg}$, and $\mathrm{He}^{*}$ in 1991, others have been made for Ar*, $\mathrm{Ca}, \mathrm{H}^{*} \mathrm{He}, \mathrm{K}, \mathrm{Li}, \mathrm{Ne}^{*}, \mathrm{Rb}$ atoms, and $\mathrm{He}_{2}, \mathrm{Li}_{2}, \mathrm{Na}_{2}, \mathrm{I}_{2}$, $\mathrm{C}_{60}, \mathrm{C}_{70}, \mathrm{C}_{60} \mathrm{~F}_{48}$, and $\mathrm{C}_{44} \mathrm{H}_{30} \mathrm{~N}_{4}$ molecules. Interferometers starting with trapped atoms have been made for $\mathrm{Ca}, \mathrm{Cs}, \mathrm{He}^{*}, \mathrm{Mg}, \mathrm{Na}$, and $\mathrm{Rb}$ and interferometers using Bose-Einstein condensates have been demonstrated with $\mathrm{Na}$ and $\mathrm{Rb}$. These lists are still growing.

\section{B. Three-grating interferometers}

The simplest white light interferometer is a MachZehnder interferometer built from three diffraction gratings. The first grating acts as a beam splitter, the second as a redirector, reversing the (transverse) momentum of the beam, and the third as a recombiner or analyzer of the interference.

\section{Mechanical gratings}

The first three-grating Mach-Zehnder interferometer for atoms was built by Keith et al. (1991) using three $0.4-\mu \mathrm{m}$-period nanofabricated diffraction gratings. Starting from a supersonic Na source with a brightness of $B$ $\approx 10^{19} \mathrm{~s}^{-1} \mathrm{~cm}^{-2} \mathrm{sr}^{-1}$ the average count rate in the interference pattern was 300 atoms per second. Since then, gratings of $100-\mathrm{nm}$ period have been used to generate fringes with up to 300000 atoms/s.

We use this design (shown in Fig. 21) to illustrate how a standing wave interference pattern is formed by the two running waves. Starting with a common beam that is incident on two gratings ( $G 1$ and $G 2$ ), one wave is formed by 0 th and 1 st order diffraction, while the other is formed by -1 st and +1 st order diffraction. The difference in momentum is thus one unit of $\hbar G \hat{x}$. So we describe the incident running waves by the functions $\psi_{1}$ and $\psi_{2} e^{i G x} e^{i \Delta \phi_{\text {int }}}$. These running waves differ in momentum explicitly by $\hbar G \hat{x}$ due to diffraction. The waves also differ in phase by $\Delta \phi_{\text {int }}$ due to different interactions along the two paths.

In the zone where these coherent running waves overlap, the atom beam intensity is

$$
\begin{aligned}
& I(x)=\left|\psi_{1}+\psi_{2} e^{i \Delta \phi_{\text {int }}} e^{i G x}\right|^{2}, \\
& I(x)=\langle I\rangle+\langle I\rangle C \cos \left(\Delta \phi_{\text {int }}+G x\right) .
\end{aligned}
$$

This interference pattern is a standing wave in space but is unchanging in time. The fringes have a period of $d$ $=G /(2 \pi)$ (just as the gratings), and a spatial offset in $\hat{x}$ (i.e., a phase) that depends on the location of the two gratings $G 1$ and $G 2$ as well as the interaction phase $\Delta \phi_{\text {int }}$. Equation (14) is a general result, and the fringes can be detected in many different ways.

The intensity pattern has a mean intensity and contrast,

$$
\langle I\rangle=\left|\psi_{1}\right|^{2}+\left|\psi_{2}\right|^{2}, \quad C=\frac{I_{\max }-I_{\min }}{I_{\max }+I_{\min }}=\frac{\psi_{1}^{*} \psi_{2}+\psi_{2}^{*} \psi_{1}}{\left|\psi_{1}\right|^{2}+\left|\psi_{2}\right|^{2}} .
$$

If one of the interfering beams is much stronger than the other, for example, $\left|\psi_{1}\right|^{2} \gg\left|\psi_{2}\right|^{2}$, then the contrast of the interference pattern scales as

$$
C \sim 2\left|\psi_{2}\right| /\left|\psi_{1}\right|=2 \sqrt{I_{2} / I_{1}} .
$$

Consequently, one can observe $20 \%$ (2\%) contrast for an intensity ratio of $100: 1 \quad\left(10^{4}: 1\right)$ in the interfering beams.

If the waves are not perfectly coherent, then the incoherent part adds to the overall intensity, and the contrast is diminished. If more than two components overlap, the situation is somewhat more complicated.

The spatial oscillations in intensity can be detected, for example, by measuring the atom flux transmitted through a third (absorbing) grating (G3). In this case $G 3$ acts as a mask to transmit (or block) the spatially structured matter wave intensity. By translating $G 3$ along $x$ one obtains a moiré filtered interference pattern which is also sinusoidal and has a mean intensity and contrast

$$
\begin{aligned}
& \langle I\rangle=\left(w_{3} / d\right)\langle\tilde{I}\rangle, \\
& C=\left[\sin \left(G w_{3} / 2\right) /\left(G w_{3} / 2\right)\right] \tilde{C},
\end{aligned}
$$

where $\tilde{I}$ and $\tilde{C}$ refer to the intensity and contrast just prior to the mask. The phase of the filtered interference pattern is given by 


$$
\phi=G\left(x_{1}-2 x_{2}+x_{3}\right)+\Delta \phi_{\text {int }},
$$

where $x_{1}, x_{2}$, and $x_{3}$ are the relative positions of gratings 1,2 , and 3 with respect to an inertial frame of reference (Schmiedmayer et al., 1997).

The phase $\phi$ will have a statistical variance $\sigma_{\phi}^{2}$ in the simplest case due to shot noise (counting statistics) (Lenef et al., 1997; Schmiedmayer et al., 1997) given by

$$
\left(\sigma_{\phi}\right)^{2} \equiv\left\langle(\phi-\langle\phi\rangle)^{2}\right\rangle=1 / C^{2} N,
$$

where $N$ is the total number of atoms counted. For discussion of how phase fluctuations depend on atom-atom interactions within the interferometer see Scully and Dowling (1993); Search and Meystre (2003); Pezze and Smerzi (2006). To minimize the uncertainty in measured phase we therefore seek to maximize $C^{2} N \propto C^{2}\langle I\rangle$ by choosing the open fractions $w_{i} / d$ for the three gratings, where $w_{i}$ is the window size for the $i$ th grating and $d$ is the grating period. The open fractions that maximize $C^{2}\langle I\rangle$ are $\left(w_{1} / d, w_{2} / d, w_{3} / d\right)=(0.56,0.50,0.37)$. With these open fractions, the theoretical value of $C=0.67$ and $\langle I\rangle / I_{\text {inc }}=0.015$. If van der Waals interactions between atoms and gratings are included, then open fractions of the first two gratings should be increased for best performance (Cronin et al., 2005).

There are in fact several different interferometers formed by the gratings. For example, the first and second orders can recombine in a skew diamond to produce another interferometer with the white fringe property. Additional mirror images of these interferometers make contrast peaks on either side of the original beam axis, as shown in Fig. 21. All those interferometers can have fringes with the same phase, and consequently one can therefore build interferometers with wide uncollimated beams which have high count rate, but lower contrast. (The contrast is reduced because additional beam components such as the zeroth-order transmission through each grating will also be detected.)

Mechanical gratings with much larger periods have been used to make interferometers in the extreme limit of nonseparated beams. We discuss these in the TalbotLau interferometer section.

For well-collimated incoming beams, the interfering paths can be separated at the second grating. For example, in the interferometer built at MIT the beams at the second (middle) grating have widths of $30 \mu \mathrm{m}$ and can be separated by $100 \mu \mathrm{m}$ (using $100-\mathrm{nm}$ period gratings and $1000 \mathrm{~m} / \mathrm{s}$ sodium atoms with $\lambda_{\mathrm{dB}}=16 \mathrm{pm}$ ). Details of this apparatus, including the auxiliary laser interferometer used for alignment and the requirements for vibration isolation, have been given by Schmiedmayer $e t$ al. (1997).

This geometry was used in the first atom interferometer with physical isolation of the spatially separated paths. Isolation was provided by inserting a $10-\mathrm{cm}-\mathrm{long}$ metal foil between the two paths, so that the electric or magnetic field or gas pressure could be varied on the left or right arm separately. This resulted in measurements of atomic polarizability (Ekstrom et al., 1995), the index of refraction due to dilute gases (Schmiedmayer et al.,
1995; Roberts et al., 2002), contrast interferometry using magnetic rephasing (Schmiedmayer et al., 1994), and diffraction phases induced by van der Waals interactions (Perreault and Cronin, 2005, 2006). In experiments not explicitly needing separated beams, this apparatus has been used to measure phase shifts due to rotations Lenef $e t$ al., 1997) and to study decoherence due to scattering photons and background gas (Chapman, Hammond, et al., 1995; Kokorowski et al., 2001; Uys et al., 2005). This apparatus was also used to perform the first separated beam experiments with molecules $\left(\mathrm{Na}_{2}\right)$ (Chapman, Ekstrom, et al., 1995).

An interferometer with similar nanogratings was developed at the MPI in Göttingen and used to measure the polarizability of $\mathrm{He}$ and $\mathrm{He}_{2}$ (Toennies, 2001).

\section{Interferometers with light gratings}

One can also build $\mathrm{MZ}$ interferometers with gratings made from light (Fig. 22). These light gratings are generally near-resonant standing waves that make speciesspecific phase gratings. Because they transmit all atoms, light gratings are more efficient than material gratings.

The third grating in a light interferometer can function in many ways to enable detection of the fringes. It can recombine atom waves so their relative phase dictates the probability to find atoms in one output port (beam) or another. Alternatively, fringes in position space can be detected with fluorescence from a resonant standing wave. Another detection scheme uses backward Bragg scattering of laser light from the density fringes. This can be used in multipath interferometers where phase shifts affect the contrast of the fringes. (We discuss such contrast interferometry in the next section.) Detecting the direction of exiting beams requires that the incident beams must be collimated well enough to resolve diffraction, and may well ensure that the beams are spatially separated in the interferometer.

Rasel et al. (1995) used light gratings in the KapitzaDirac regime with a 5- $\mu$ m-wide collimated beam. Many different interferometers were formed, due to symmetric KD diffraction into the many orders. Two slits after the interferometer served to select both the specific interferometer and the momentum of the outgoing beam (ports 1 and 2 in Fig. 22). Fringes with 10\% contrast show complementary intensity variations, as expected from particle number conservation in a MZ interferometer with phase gratings.

It is even more efficient to use Bragg diffraction because no atoms are lost to "unwanted" orders. Giltner $e t$ al. (1995a) used Bragg diffraction and a $\mathrm{Ne}^{*}$ beam and obtained contrast of $C=63 \%$. Higher-order Bragg diffraction was also used to demonstrate smaller period interference fringes shown in Fig. 22. A Bragg scattering interferometer for $\mathrm{Li}$ atoms with a contrast of 0.84 and a count rate of $17 \mathrm{kc} / \mathrm{s}$ was used to measure the polarizability of Li atoms (Delhuille, Champenois, et al., 2002; Miffre et al., 2006b, 2006c). 
a)

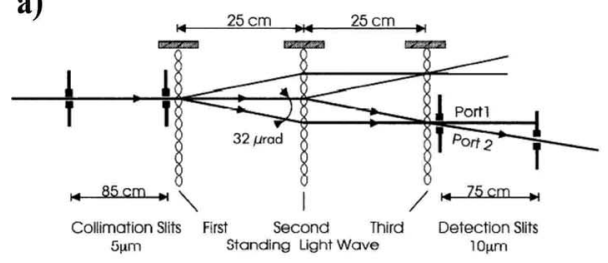

b)

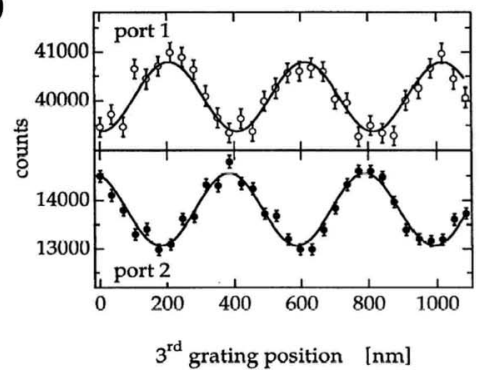

c)

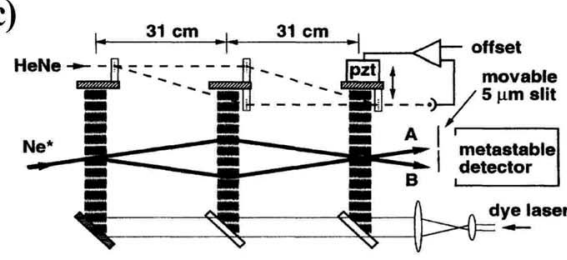

d)

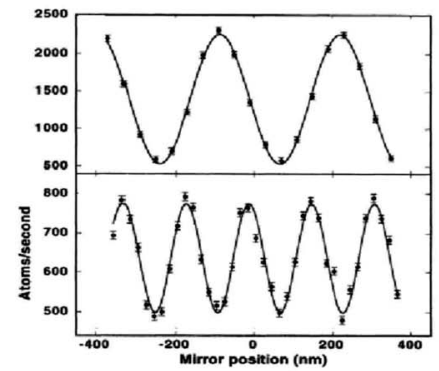

e)

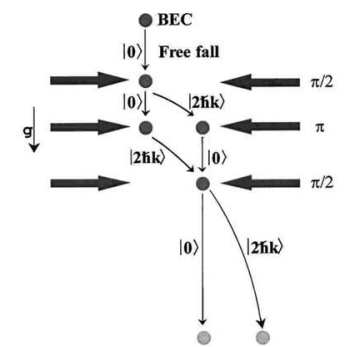

f)
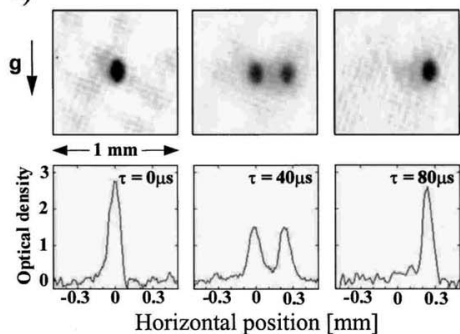

FIG. 22. Atom interferometers based on three standing waves of light. (a) Atom beam and three Kapitza-Dirac gratings. (b) Atom interference patterns for output ports demonstrate complementary intensity variations. This is a consequence of atom number conservation. Figures (a) and (b) from Rasel et al., 1995. (c) Interferometer based on three Bragg gratings. Dashed line shows the path of auxiliary optical interferometer used for stabilization. (d) Intensity fluctuations in beam A vs position of the Bragg gratings. For second-order Bragg diffraction, fringes of half the period are formed. Figures (c) and (d) from Giltner et al., 1995a. (e) Schematic of the $\pi / 2-\pi-\pi / 2$ Bragg interferometer for atoms in a BEC falling from a trap. (f) Absorption images and density profiles demonstrating different outputs of the interferometer. Figures (e) and (f) from Torii et al., 2000.

\section{Time-domain and contrast interferometers}

Light gratings can easily be turned on and off, allowing one to control the interaction times of atoms with the three gratings. Thus, independent of initial longitudinal momentum, all atoms will see an equal interaction and will subsequently separate equally (since they have the same momentum transferred by the grating). Such interferometers are valuable in precision experiments since time is measured accurately. This consideration also applies to optical Raman pulses, which will be discussed later. Torii et al. (2000) made a three-grating interferometer for ultracold atoms by pulsing gratings thrice in time. These pulsed gratings are turned on for a duration long enough to produce Bragg diffracted momentum states. This duration does not affect the precise timing between interactions, but is long enough that diffraction in time is unimportant. Their fringes were read out in momentum space by measuring the atom cloud position in absorption images taken shortly after the third grating pulse. Atoms released from a BEC were used insuring that the momentum spread of the cloud was smaller than a photon recoil momentum $\hbar k_{\mathrm{ph}}$ thus allowing resolution of the output states. More examples of time-domain interferometers based on three diffraction gratings include Gupta et al. (2002) and Wang et al. (2005).

Gupta et al. (2002) used one Kapitza-Dirac pulse followed by a second-order Bragg pulse to make an interferometer with three paths as shown in Fig. 23. One can understand this arrangement as two separate two-path interferometers whose density fringes overlap. Because the phase of each two-path interferometer changes in time in opposite directions, the two density gratings move in and out of register as time and phase increase, hence the contrast oscillates rapidly with time. This interferometer has been used to measure $h / m_{\mathrm{Na}}$ to a precision of $7 \mathrm{ppm}$. This demonstrates the utility of contrast interferometry in which measurements of contrast, not
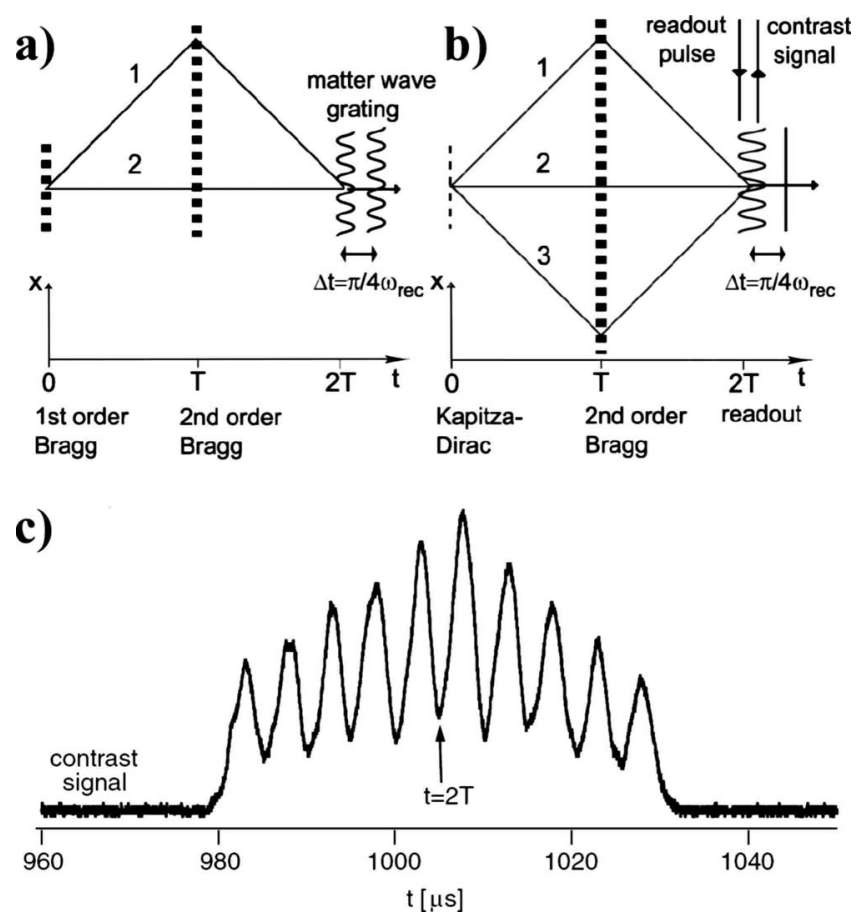

FIG. 23. Contrast interferometry. (a) Space-time representation of a two-path interferometer that is sensitive to the photon recoil phase. (b) The three-path geometry. The overall fringes have large contrast at $2 T$ and zero contrast at $2 T$ $+\pi / 4 \omega_{\text {rec }}$. Bottom: Typical single-shot signal from the contrast interferometer. From Gupta et al., 2002. 
phase, are used. Contrast interferometry was pioneered by Schmiedmayer et al. (1994) where interference patterns from atoms with different magnetic sublevels moved in and out of register.

This contrast interferometer design offers several advantages compared to phase measurements made with a regular interferometer. First, the fringe phase can be accurately determined in a single "shot," eliminating effects of shot-to-shot atom intensity fluctuations. Second, most experimental sources of phase noise affect each two-path interferometer in the same way they move the fringes but do not change the contrast. For example, measurements with the interferometer by Gupta et al. (2002) were nearly insensitive to vibrations, rotation, accelerations, and magnetic field gradients. A relative phase shift between the two interferometers can be caused, however, by diffraction phases Buchner et al. (2003). If the Kapitza-Dirac pulse causes a phase shift between the 0th and 1st diffraction order, then the contrast does not peak at exactly $2 T$, where $T$ is the time between diffraction grating pulses. Hence fluctuations in the intensity of light used for the KD pulse can then lead to fluctuations in the time at which the total contrast peak is visible.

Gupta et al. (2002) detected the contrast of the fringes in space by measuring the intensity of reflected (Bragg diffracted) light probing the fringes in space. The intensity of reflected light can be continuously monitored as the two sets of interference fringes pass through each other in time. This causes oscillations in the intensity of reflected light as shown in Fig. 23.

\section{Talbot-Lau (near-field) interferometer}

We now turn to near-field interferometers. As discussed in Sec. II.D, a high degree of spatial coherence is needed to create recurring self-images of a grating due to near-field diffraction (the Talbot effect). But completely incoherent light can still produce fringes downstream of a grating pair (the Lau effect). When two gratings with equal period $(d)$ are separated by a distance $L_{1}$, the Lau fringe contrast is maximum at a distance beyond the second grating of

$$
L_{2}=\frac{L_{1} L_{T} n / 2 m}{L_{1}-L_{T} n / 2 m}
$$

where $L_{T}=2 d^{2} / \lambda_{\mathrm{dB}}$ is the Talbot length and the integers $n$ and $m$ refer to the $n$th revival of the $m$ th higher-order Fourier image. The fringe period is then

$$
d^{\prime}=d\left(L_{2}+L_{1}\right) / m L_{1}
$$

If a third grating is used as a mask to filter these fringes, then a single large-area integrating detector can be used to monitor the fringes. This three-grating arrangement is a Talbot-Lau interferometer (TLI). A typical TLI uses three identical gratings and $L_{1}=L_{2}=L_{T} / 2$ with $n=1$ and $m=2$.

The first grating can be regarded as an array of small but mutually incoherent sources of diverging waves. Shortly after, the second grating near-field diffraction

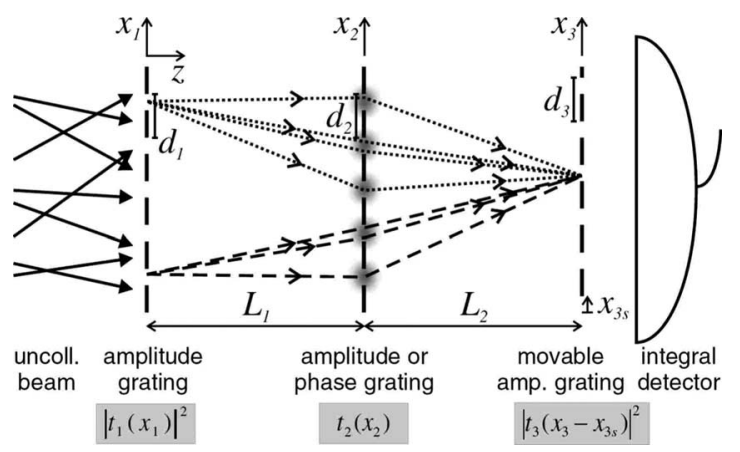

FIG. 24. A sketch of the Talbot-Lau interferometer setup consisting of three gratings. The first grating is illuminated by an uncollimated molecular beam. Coherent interference occurs between all paths that originate from one point at the first grating and meet at the a point on the third grating. By varying the grating position $x_{3}$, a periodic pattern in the molecular distribution can be detected. The second grating can be replaced by a light grating (Gerlich et al., 2007; Hornberger et al., 2009). From Brezger et al., 2003.

causes any shadow effects to become blurred out. At a distance $L_{2}$ from the second grating, spatial structure in the intensity starts to re-emerge. The intensity oscillations observed with a TLI are not a ray-optics phenomenon; they are due to wave interference for the multiple paths shown in Fig. 24. Evidence for this is that $L_{2}$ depends on $\lambda_{\mathrm{dB}}$ (and hence a fairly monochromatic velocity distribution is needed for optimum contrast). The second grating can be a phase grating, but the first and third gratings must be amplitude gratings. The theory of this interferometer has been discussed by Clauser and Reinisch (1992); Clauser and Li (1994b, 1997); Batelaan et al. (1997); Brezger et al. (2003); Hornberger et al. (2009).

A feature of a TLI is that the contrast is unaffected by the beam width. A large transverse momentum spread in the beam is also tolerated. Hence much larger count rates can be obtained with a TLI.

Furthermore, in a TLI the relationship $L_{1}=L_{2}=L_{T} / 2$ means that the maximum grating period is $d<\sqrt{L_{1} \lambda_{\mathrm{dB}}}$ $\sim M^{-1 / 4}$, where $M$ represents the mass for a thermal beam. In comparison, for a MZI with resolved paths the requirement is $d<\lambda_{\mathrm{dB}} L / \Delta x \sim M^{-1 / 2}$ where $\Delta x$ is the width of the beam and $L$ is the spacing between gratings. Thus the TLI design is preferable for massive particles.

A Talbot-Lau interferometer was first built for atoms by Clauser and Li (1994b) using a slow beam of potassium atoms. The experiment used gratings with a period of $d=100 \mu \mathrm{m}$, and a count rate of $\langle I\rangle=4 \times 10^{7}$ atoms $/ \mathrm{s}$ was achieved. The source brightness was 2500 times weaker than in the three grating Mach-Zehnder interferometer of Keith et al. (1991), but the signal was about 3000 times stronger. Because of its attractive transmission features, and the favorable scaling properties with $\lambda_{\mathrm{dB}}$, the TLI has been used to observe interference fringes with complex molecules such as $\mathrm{C}_{60}, \mathrm{C}_{70}, \mathrm{C}_{60} \mathrm{~F}_{48}$, and $\mathrm{C}_{44} \mathrm{H}_{30} \mathrm{~N}_{4}$ (Brezger et al., 2002; Hackermuller, Uttenthaler, et al., 2003; Gerlich et al., 2007; Hornberger et 
al., 2009). Of course, the TLI does not separate the orders-indeed, components of the wave function are only displaced by one grating period at the Talbot length. Even though the TLI interferometer cannot offer separated paths, it is still sensitive to inertial forces, decoherence, and field gradients (Clauser and Li, 1994a; Hornberger et al., 2003; Hackermuller et al., 2004; Berninger et al., 2007).

Cahn et al. (1997) used the phrase "time domain interferometry" to describe a Talbot-Lau interferometer that consists of two (Kapitza-Dirac) gratings pulsed in time, and renders interference fringes in position space. A third pulse of light, a traveling wave, was Bragg reflected by the atomic density pattern and thus served as the detection scheme for fringes. The atom fringe contrast (and backscattered probe light) oscillates with the characteristic Talbot time $\tau_{T}=\biguplus_{T} / v=2 d^{2} m / h$, and this readout mechanism demonstrates $100 \%$ contrast even with an "uncollimated cloud" of atoms. Talbot-Lau interferometry with (pulsed) light gratings has also been explored by Deng et al. (1999); Cohen et al. (2000); Turlapov et al. (2003, 2005); Wu, Su, et al. (2005); Weel et al. (2006).

\section{Interferometers with path-entangled states}

In some interferometers, the internal state of the atoms depends on the path through the interferometer. Hence the state of the atom is entangled with the path. This usually occurs when the rf or laser photons that cause a transition between internal states also impart momentum, thus creating such entanglement.

Such entanglement has implications both for what the interferometer can measure and for how the interference can be detected. Detection is the more obvious; if recombination results in oscillations between two internal states, then state-sensitive detection can reveal the fringes without need for the atom paths to be spatially resolved. The influence of having different internal states in the middle of the interferometer is more subtle. Many atomic properties such as polarizability and scattering lengths depend on the state; hence such interferometers naturally measure the difference of that property between the states which is generally less informative than the property in one state.

\section{Optical Ramsey-Bordé interferometers}

When a traveling wave of resonant light intersects a two-level atom, the atom is put into a superposition of ground and excited states in which the photon absorbed in promoting the atom to the excited state has added its momentum to that of the ground state, resulting in a differential momentum of $\hbar k_{\mathrm{ph}}$ between ground and excited states [Fig. 25(a)],

$$
|a, p\rangle \rightarrow \sin (\theta)|a, p\rangle+\cos (\theta)\left|b, p+\hbar k_{\mathrm{ph}}\right\rangle .
$$

Bordé's seminal 1989 paper that optical Ramsey spectroscopy by four traveling laser fields is an atom interferometer when taking the momentum transfer in the

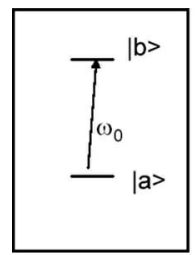

(a)

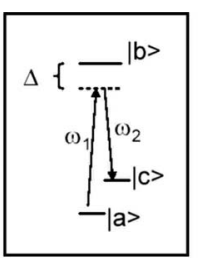

(b)

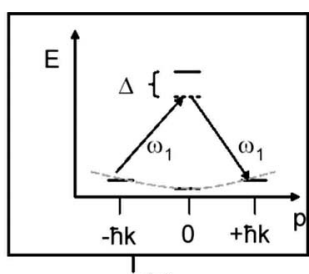

(c)
FIG. 25. Different schemes used to place atoms in a superposition of momentum states. (a) Superposition with a metastable state using a $\pi / 2$ pulse. (b) Stimulated Raman transition with two light fields. (c) Bragg scattering with monochromatic light. $\Delta$ is the detuning from resonance. The dashed curve is the kinetic energy $p_{\text {light }}^{2} / 2 m$.

excitation process into account (Bordé, 1989). Such an experiment is now often called a Ramsey-Bordé interferometer. In comparison, the classic Chebotayev paper (Chebotayev et al., 1985) focused on Kapitza-Dirac or Bragg diffraction gratings that preserve atoms' internal state. A unified description of these cases can be found in Bordé (1997).

If the excitation is on resonance, the fraction of amplitude that is deflected by the transition is determined by the pulse area $\theta=\int \Omega_{1} d t$, where $\Omega_{1}=\mathbf{d}_{\mathrm{ab}} \cdot \mathbf{E}_{0} / \hbar$ is the bare Rabi frequency. A Ramsey-Bordé $\pi / 2$ pulse (named for the condition $\theta=\pi / 2$ ) results in an equal splitting of the amplitude between states $|a\rangle$ and $|b\rangle$ by resonant light. If the excitation is detuned by $\Delta$,

$$
P_{b}(t)=\frac{1}{2}\left(\Omega_{1} / \Omega_{R}\right)^{2}\left[1-\cos \left(\Omega_{R} t\right)\right],
$$

where $\Omega_{R}=\sqrt{\Omega_{1}^{2}+\Delta^{2}}$ is the generalized Rabi frequency. When the detuning grows the oscillations become more rapid and less complete.

For an optical Ramsey-Bordé interferometer to work, the lifetime of the excited state must be comparable to the transit time through the interferometer in order to avoid coherence-destroying spontaneous decay of state $|b\rangle$ (see Sec. IV.B on decoherence). Consequently, optical Ramsey-Bordé interferometers are generally used with long-lived, metastable excited states such as the $1 S-2 S$ transition in $\mathrm{H}$, or the lowest-lying intercombination lines of Mg or Ca (Morinaga et al., 1989; Gross et al., 1998; Ruschewitz et al., 1998; Oates et al., 1999).

In the four zone Ramsey-Bordé interferometer atoms passing through the first laser beam are put in a superposition of internal states $|a\rangle$ and $|b\rangle$. Several possible paths exit this apparatus, but only the paths shown in Fig. 26 cause interference fringes in the populations (outputs I and II of Fig. 26). Oscillations in the state $|b\rangle$ population are controlled by the phase of the laser at each of the four zones, therefore the simplest way to produce fringes is to adjust the laser frequency. Additional phase shifts in the fringes can be caused by any interaction that affects the internal states differentially, for example, magnetic fields. Because of the photon recoil, the two paths are also separated in space and are therefore sensitive to field gradients and inertial displacements. 

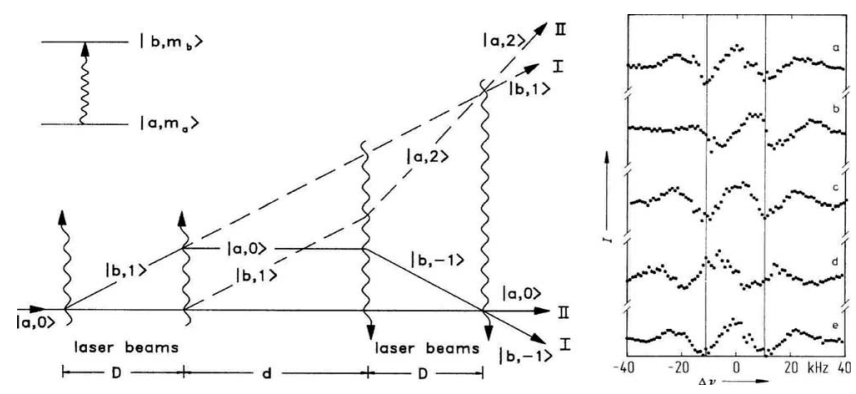

FIG. 26. Ramsey-Bordé interferometer. Left: In the first interaction zone the matter wave is coherently split into two partial waves with internal states $\left|a, m_{a}\right\rangle$ and $\left|b, m_{b}\right\rangle$ corresponding to energy levels $a$ and $b$, respectively, and the number $m$ of photon momenta transferred to the atom. Right: Fringe shifts due to rotation at different rates. From Riehle et al., 1991.

This four zone design of a Ramsey-Bordé interferometer was realized by Riehle et al. (1991) who also demonstrated the linear increase of phase shift with rotation rate $\Omega$. The data shown in Fig. 26 are the first demonstration of the Sagnac effect for atom interferometers.

Since then many Ramsey-Bordé interferometers were employed for $\mathrm{H}, \mathrm{Mg}$, and $\mathrm{Ca}$ atoms and $\mathrm{I}_{2}$ molecules for precision experiments such as high-resolution spectroscopy (Sengstock et al., 1993; Kisters et al., 1994; Gross et al., 1998; Huber et al., 1998; Oates et al., 1999; Heupel et al., 2002; Wilpers et al., 2002; Sterr et al., 2004; Degenhardt et al., 2005; Keupp et al., 2005) and fundamental studies such as geometric phases; and light shift potentials (Muller et al., 1995; Mei et al., 2000; Yanagimachi et al., 2002), transition Stark shifts (Morinaga et al., 1993; Rieger et al., 1993) and multiple beam high-finesse atom interferometry (Weitz et al., 1996; Hinderthur et al., 1997, 1999; Ruschewitz et al., 1998), and molecule interferometry (Bordé et al., 1994).

\section{Raman interferometry}

A similar beam splitter can be implemented using Raman transitions between two low-lying (e.g., hyperfine) states in a three-level atoms [Fig. 25(b)]. The superposition is now between two long-lived states and can be driven with lasers tuned off-resonant from the excited state so that spontaneous emission is no obstacle to coherence time. For building an atom interferometer one has to transfer momentum during the Raman transition. Consequently, two counter propagating running waves ${ }^{10}$ $\left(\omega_{1}\right.$ and $\left.\omega_{2}\right)$ of light with frequencies tuned to Raman resonance $\left(\hbar \omega_{1}-\hbar \omega_{1}=E_{|a\rangle}-E_{|c\rangle}=\Delta E_{\mathrm{hf}}\right)$ are used to stimulate Raman transitions between two hyperfine states $|a\rangle$ and $|c\rangle$ [Fig. 25(b)]. Absorption from one light

\footnotetext{
${ }^{10}$ Counterpropagating light beams make Doppler sensitive transitions that are highly selective for atomic velocity; copropagating light beams make Doppler insensitive transitions. Doppler sensitive Raman transitions can prepare atoms with a momentum uncertainty of less than a photon recoil (Kasevich et al., 1991).
}

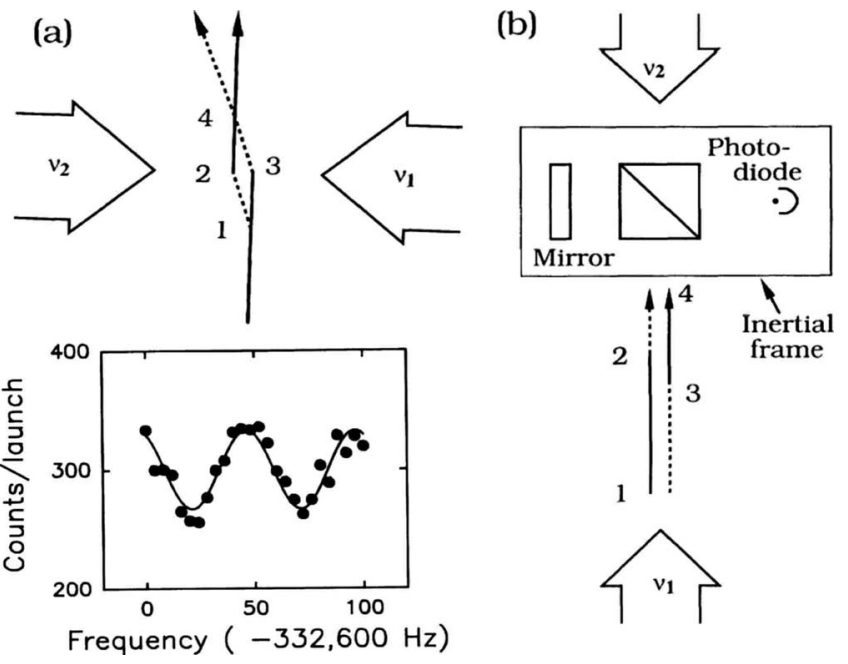

FIG. 27. Raman pulse interferometer. (a) Transverse splitting and (b) longitudinal splitting of atoms with a $\pi / 2-\pi-\pi / 2$ pulse interferometer. The mechanical recoil from the first $\pi / 2$ pulse (at position 1) coherently splits the atomic wave packet. The $\pi$ pulse (positions 2 and 3) redirects each wave packet's trajectory. By adjusting the phase of the second $\pi / 2$ pulse (position $4)$, the atom can be put into either $|1\rangle$ or $|2\rangle$. In the experiment, the atoms were prepared in the $|1\rangle$ state (solid lines) and detected in the $|2\rangle$ state (dashed lines). Bottom: Interferometer fringes are observed by scanning the frequency of the Raman laser beams. From Kasevich and Chu, 1991.

beam and stimulated emission into the other gives atoms a momentum kick of $\hbar \Delta k=\hbar k_{1}+\hbar k_{2} \sim 2 \hbar k_{\mathrm{ph}}$. Since the hyperfine splitting $\Delta E_{\mathrm{hf}} \ll \hbar \omega_{1,2}$ is much smaller then the energy of either of the photons $\left(\hbar \omega_{1,2}\right)$ the momentum transfer can be approximated by $2 \hbar k_{\mathrm{ph}}$ (reduced by the cosine of the half angle between light beams).

Transfer of amplitude from $|a\rangle$ to state $|c\rangle$ mimics the dynamics of a driven two level system with coupling frequency equal to the product of the individual Rabi frequencies divided by $\Delta$ [see Fig. 25(b)].

An alternative to Raman transitions is stimulated adiabatic rapid passage described by Gaubatz et al. (1990) and Bergmann et al. (1998). This process is more controllable since it does not depend so critically on laser power. The method is based on adiabatic change of a "dark state" and has the disadvantage that only one superposition of the two states survives (the other decays spontaneously) hence its application to interferometry gives only one output state.

Starting with laser-cooled sodium atoms launched from a trap, Kasevich and Chu (1991, 1992) demonstrated an interferometer based on stimulated Raman transitions by employing a $\pi / 2-\pi-\pi / 2$ sequence (Fig. 27). The $\pi / 2$ pulses act as beam splitters, and the $\pi$ pulse acts to completely change the state and reverse the differential momentum in each arm of the interferometer, in essence a three-grating interferometer. Similar to the Ramsey-Bordé interferometer, the paths have internal state labels. The interference is detected as oscillations in the population of the different internal states after the interferometer, as measured with state- 
sensitive fluorescence or ionization. Since the gratings are pulsed in time such an arrangement is a time domain atom interferometer. These experiments employed atomic fountains for $\mathrm{Na}$ (Kasevich and $\mathrm{Chu}, 1991,1992)$ or Cs atoms (Peters et al., 1999) to permit longer interaction times. In the first experiments (with $\mathrm{Na}$ ) a time delay between pulses of $100 \mathrm{~ms}$ gave a wave-packet separation of $6 \mathrm{~mm}$ [cf. $66 \mu \mathrm{m}$ for thermal beams with fabricated gratings (Keith et al., 1991)]. Chu and coworkers have refined this technique to get high contrast $(C=65 \%)$ fringes with a count rate of $\langle I\rangle=10^{6}$ atoms $/ \mathrm{s}$. This allowed measurements of $g$ at the part-per-billion level (Peters et al., 1999, 2001). The theory of this interferometer has been discussed by Young et al. (1997) and Kasevich and Chu (1992). Higher-order Raman transitions can be stimulated with multiple pulses, and momentum differences of $60 \hbar k_{\mathrm{ph}}$ have been used for interferometry (Weiss et al., 1993).

A beam experiment using the same kind of Raman transitions was used by Gustavson et al. $(1997,2000)$ to measure rotation rates, and achieved short-term sensitivity to rotations of $\left(6 \times 10^{-10} \mathrm{rad} / \mathrm{s}\right) / \sqrt{\mathrm{Hz}}$ as discussed in Sec. V.A. In this configuration, the gratings were not pulsed, so this was a space-domain interferometer.

We discuss several other applications of this kind of interferometer, like precise measurements of gravity gradients (Snadden et al., 1998; McGuirk et al., 2002), Newton's constant $G$, and the value of $\hbar / M$ (Weiss et al., 1993; Peters et al., 1997), in Sec. V on precision measurements.

\section{Longitudinal interferometry}

The standard description of Ramsey's separated fields experiment treats the longitudinal motion classically and as being the same for both states. This is obviously incorrect if the states have different magnetic moments and the beam passes into a region with a different magnetic field-the field gradient puts a different force on components with different magnetic moments, and could even reflect one state but not the other. Another source of longitudinal energy shift is excitation by rf radiation whose frequency is below (or above) resonance: the remaining energy to excite the atom comes from (or goes into) the kinetic energy of the excited-state component. In fact, the transition can be made by a gyrating field with zero temporal frequency, especially if the beam is moving fast so that the spin cannot follow the field as it passes. We discuss these cases below.

\section{Stern-Gerlach interferometry}

While a Stern-Gerlach magnet can entangle an atom's spin and momentum transverse to the beam velocity, it is difficult to redirect and recombine amplitudes along these two paths (Englert et al., 1988; Schwinger et al., 1988; Scully et al., 1989; Reinisch, 1999). In a different geometry, atoms in a beam can be split longitudinally, so that components of each atom are separated along the direction of the beam velocity. This is easy to accom- a)

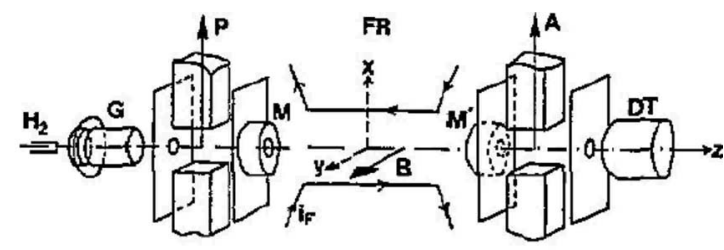

b)

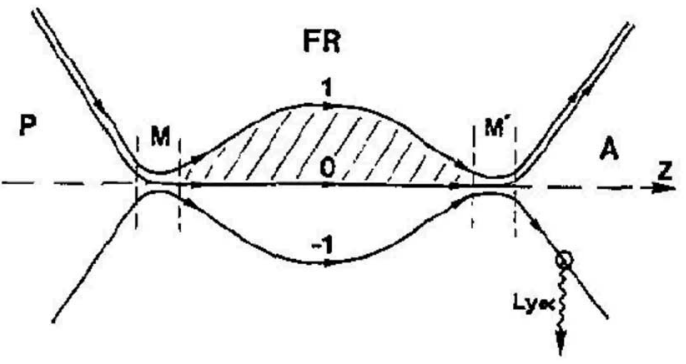

c)

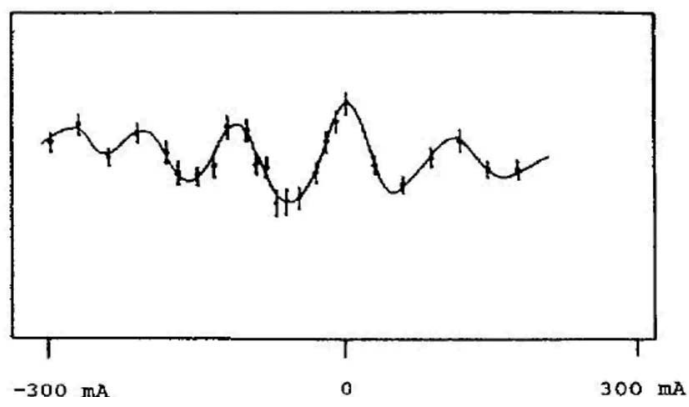

FIG. 28. Longidudinal Stern-Gerlach interferometer. (a) Experimental setup: source $G$, polarizing and analyzing magnetic fields $\mathrm{P}$ and $\mathrm{A}$, mixers $\mathrm{M}$ and $M^{\prime}$, frame FR with current $i_{F}$ is creating a magnetic field $B$, detector DT. (b) The energy landscape for the Zeeman states $(-1,0,1)$ of $H^{*}\left(2 s_{1 / 2}, F=1\right)$ along axis $Z$. (c) Interference pattern obtained with a transverse magnetic field in region FR. Adapted from Chormaic et al., 1993.

plish, and has the advantage (for interferometry) that the two paths overlap (Miniatura et al., 1991; Robert et al., 1991; DeKieviet et al., 1995).

A longitudinal Stern-Gerlach interferometer from Robert et al. (1991) is shown in Fig. 28. A partially polarized beam of metastable hydrogen atoms in the state $2 s_{1 / 2}, F=1\left(\lambda_{\mathrm{dB}}=40 \mathrm{pm}\right)$ is prepared in a linear superposition of magnetic sublevels by a nonadiabatic passage (projection on the new eigenstates) through a magnetic field perpendicular to the atomic beam. The magnetic field gradient along the beam shifts the longitudinal momentum of different atomic center of mass wave packets proportionally to their magnetic state. Next, the different magnetic sublevels enter a constant magnetic field region, and after $10 \mathrm{~cm}$ are recombined again in a region identical to the one used as a beam splitter. Finally, an analyzing magnetic field selects a particular magnetic polarization, whose intensity is then measured by detecting Lyman- $\alpha$ photons emitted in the decay of the $2 p_{1 / 2}$ state to the ground state. A typical interference pattern is shown in Fig. 28 (Robert et al., 1992; Chormaic et al., 1993).

Interference fringes are obtained in the beam intensity by changing the magnetic field strength, and arise 
from the different potentials experienced by the magnetic sublevels in the region of constant magnetic field. The longitudinal Stern-Gerlach interferometer was applied to demonstrate the effect of topological phases on the atomic wave function for a nonadiabatic cyclic evolution (Miniatura et al., 1992).

\section{Spin echo}

Along similar lines, DeKieviet et al. (1997) developed an atomic beam spin-echo (ABSE) interferometer with ${ }^{3} \mathrm{He}$ atoms. Following the Stern-Gerlach arrangement described above one can apply a reversed field (or a $\pi$ pulse) and extend this type of interferometer with an "echo," in complete analogy to the spin-echo technique used for neutrons (Mezei, 1993). The ${ }^{3} \mathrm{He}$ ABSE has the advantage that ${ }^{3} \mathrm{He}$ can reflect from a surface at grazing incidence, and therefore can be applied as interferometric probe of surfaces (DeKieviet et al., 1995, 1997; Zielonkowski et al., 1998; DeKieviet, Dubbers, Hafner, et al., 2000).

In a quantum mechanical picture, the Larmor precession can be viewed as a magnetic birefringence [Fig. 29(a)]. Note that the Zeeman states $|\rightarrow\rangle$ and $|\leftarrow\rangle$ arrive with some time delay $\tau_{\mathrm{SE}}$ (spin-echo time) at the scattering center, which allows time-correlation spectroscopy of the sample. The contrast in the measured echo signal depends on the degree to which the Zeeman states are scattered coherently. For nonstatic samples this will depend on $\tau_{\mathrm{SE}}$ [see Fig. 29(b)]. The interference contrast directly measures the correlation function $I\left(\mathbf{q}, \tau_{\mathrm{SE}}\right)$ in the time domain, which is the Fourier transform of the scattering function $S\left(\mathbf{q}, \tau_{\mathrm{SE}}\right)$ ( $\mathbf{q}$ is determined by the scattering geometry). ABSE with ${ }^{3} \mathrm{He}$ atoms has been successfully applied in surface science as an alternative to timeof-flight experiments. The spin-echo experiment is much more sensitive, with an energy resolution extending into the sub-neV range.

ABSE is not restricted to longitudinal interferometry; depending on the direction of the magnetic field gradient, the paths of the magnetic substates may diverge perpendicular to the beam direction. Using atomic hydrogen with a de Broglie wavelength of around $100 \mathrm{pm}$, Lang (1998) measured a transverse spin-echo interference signal for path separations exceeding $100 \mathrm{~nm}$.

In an entirely optical setup a spin echo was demonstrated through hyperfine pumping a thermal beam of lithium atoms (Zielonkowski et al., 1998). Here the spin echo is induced via a "virtual magnetic field," by applying a short pulse of intense, far detuned photons. The light causes a shift in the hyperfine levels that depends linearly on the quantum number $m_{F}$, just like Zeeman splitting (Cohen-Tannoudji and Dupont-Roc, 1972; Rosatzin et al., 1990).

\section{Longitudinal rf interferometry}

Dhirani et al. (1997) showed that a detuned radiofrequency field constitutes a beam-splitter in longitudinal momentum space for atoms. If an atom makes a
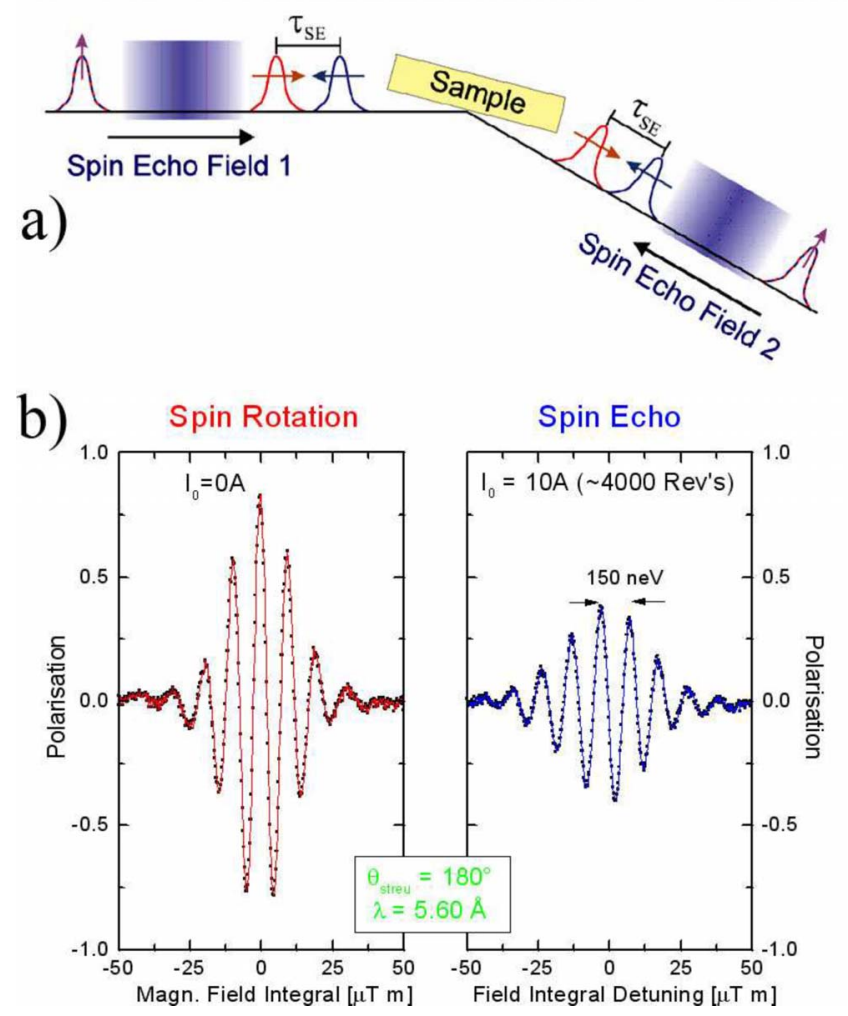

FIG. 29. (Color online) Atomic beam spin-echo interference technique for a spin-1/2 particle. (a) Schematic of setup: upon entering spin-echo field 1 , the linearly polarized wave packet $|\uparrow\rangle$ is split into two polarizations $|\rightarrow\rangle$ and $|\leftarrow\rangle$, having different energies in the longitudinal magnetic field. By inverting the direction of the spin-echo field 2 with respect to the first one, the Zeeman states $|\rightarrow\rangle$ and $|\leftarrow\rangle$ exchange roles (like a $\pi$ flip). At the end they overlap and coherently add up to $|\downarrow\rangle$ or $|\uparrow\rangle$ depending on the phase shift. The initial linearly polarized wave packet reappears as an echo. (b) Experimental ABSE data using a $4-\mathrm{K}$ beam of ${ }^{3} \mathrm{He}$ atoms. The beam averaged linear polarization as a function of the spin-echo field. Spin rotation: when the spin-echo field is off, the interference pattern is generated through Stern-Gerlach interferometry. Spin echo: when the same (but inverted) current is applied through both spin-echo coils an echo appears. Figure courtesy of M. DeKieviet.

transition to an excited quantum state by absorbing a quantum of off-resonant rf radiation, then its longitudinal velocity is changed such that total energy is conserved.

Using two such beam splitters, Smith et al. (1998) constructed a longitudinal atom interferometer in a generalization of Ramsey's separated oscillatory fields (SOF) configuration. This technique is referred to as differentially tuned separated oscillatory fields (DSOF). Oscillations in excited-state population in both time and space occur after an atom beam passes the two DSOF regions. To measure the phase and amplitude of these oscillations, a third oscillatory field and a state selective detector were used, as shown in Fig. 30. This interferometer is well-suited to studying the longitudinal coherence properties of matter-wave beams. Scanning the position of 
a)

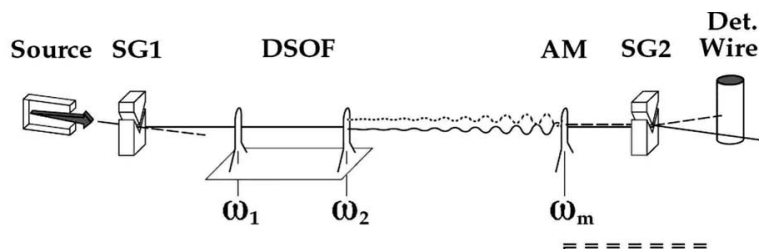

b)

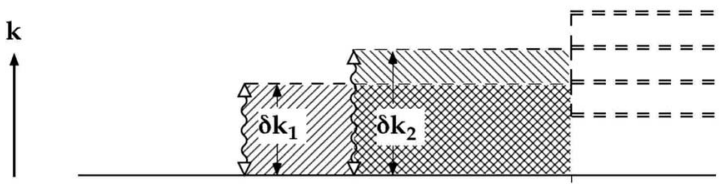

c)

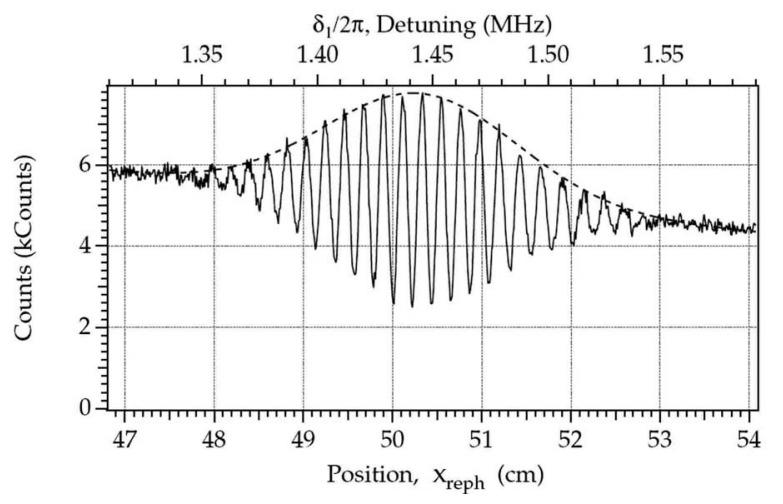

FIG. 30. Longitudinal rf interferometer: (a) schematic. Coils at longitudinal positions $x_{1}$ and $x_{2}$ with oscillatory fields at $\omega_{1}$ and $\omega_{2}$, respectively, make the differentially tuned separated oscillatory fields (DSOF). The amplitude modulator coil is located at $x_{m}$. The ground state is selected by upstream Stern-Gerlach magnet SG1, and the excited state by SG2. (b) Wave number $k$ vs the longitudinal position $x$ for states that are detected. Dashed lines indicate the excited internal state, and hatched areas denote the differential phases accrued by atoms excited at $x_{1}\left(x_{2}\right)$. (c) Fringes demonstrated with the DSOF system and an additional AM modulator. Adapted from Smith et al., 1998.

the third oscillating field demonstrates that the DSOF system can produce or detect coherent momentum superpositions.

The envelope of the fringes in space, Fig. 30, indicates the velocity width of the atom beam was $36 \pm 4 \mathrm{~m} / \mathrm{s}$ and the fringe period in space indicates the most probable beam velocity was $1080 \pm 3 \mathrm{~m} / \mathrm{s}$. An argon seeded supersonic source of sodium atoms was used.

The same DSOF arangement was used to demonstrate the absence of off-diagonal elements in the densiy matrix in a supersonic atom beam, thus showing that there are no coherent wave packets emerging from this source (Rubenstein, Dhirani, et al., 1999). In a further demonstration, the DSOF longitudinal interferometer was used to measure the complete longitudinal density matrix of a deliberately modulated atom beam (Dhirani et al., 1997; Rubenstein, Kokorowski, et al., 1999). A fully quantum mechanical treatment of this system was developed for this analysis (Kokorowski et al., 1998), and these experiment are summarized by Kokorowski et al. (2000).

\section{Stückelberg interferometers}

Stückelberg oscillations occur when a level crossing for internal states acts as a beam splitter. For example, if a)
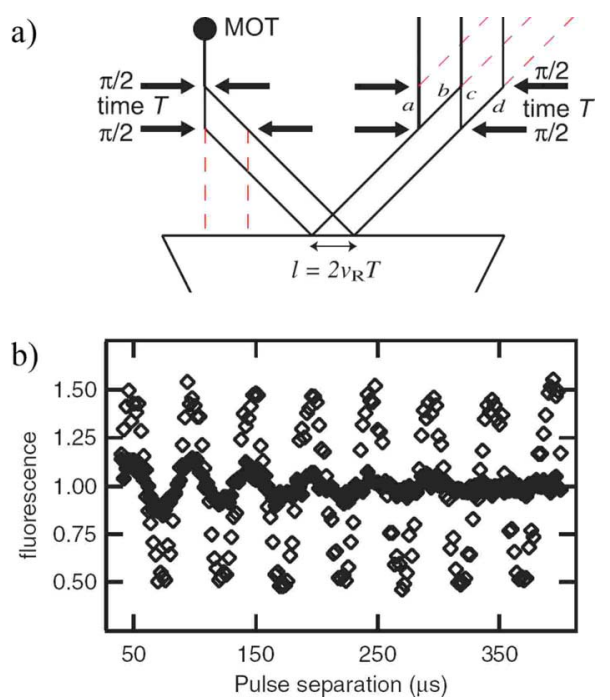

FIG. 31. (Color online) An atom mirror inside an interferometer. (a) Diagram of the interferometer. The arrows represent Raman $\pi / 2$ pulses which create superpositions of different internal states and momenta. The atomic mirror is an evanescent wave at the surface of a glass prism represented by the trapezoid. The letters $a, b, c$, and $d$, label the four possible paths. (b) Filled symbols show fringes obtained by scanning the pulse separation $T$ with paths reflected from the mirror. For reference, open symbols show fringes from the same interferometer operating in free space without mirror. From Esteve et al., 2004.

an atom can change its internal state on the way either to or from a reflecting surface, then two amplitudes for making a transition will interfere. Oscillations in the probability for state-changing atomic reflection can thus be regarded as longitudinal interferometry. One application is to survey the van der Waals potential near surfaces (Cognet et al., 1998; Marani et al., 2000).

\section{E. Coherent reflection}

Here we list more experiments in which reflected de Broglie waves are demonstrably coherent. Shimizu demonstrated reflection mode holograms (Shimizu and Fujita, 2002b) and a reflection-mode double-slit experiment (Kohno et al., 2003). Westbook used a Ramanpulse atom interferometer to study coherent reflection from an evanescent light field (Esteve et al., 2004), as shown in Fig. 31. Dekieviet used a longitudinal SternGerlach interferometer to study quantum reflection of ${ }^{3} \mathrm{He}$ (Druzhinina and DeKieviet, 2003). Zimmermann used a chip-integrated magnetic grating to diffract and interfere reflected BECs (Gunther et al., 2005, 2007; Fortagh and Zimmermann, 2007).

\section{F. Confined atom interferometers with BECs}

In this section we discuss a different type of interferometer, where the atoms are confined in a threedimensional potential well during the splitting of their wave function and application of the interaction. In this 
new type of splitting process, the single trap holding the ultracold gas of atoms (or BEC) is continuously deformed into two adjacent potential wells, each containing a part of the wave function. Thus the splitting step in the interferometer occurs in position space.

This splitting in position space is in sharp contrast to previously discussed atom and most optical interferometers, in which the splitting process occurs in momentum space. Using diffraction gratings or pulses of light transfers momentum; similarly a partially reflecting surface changes the momentum of the reflected, but not the transmitted, beam. The two maxima then separate to a varying extent in position space only because the wave is split in momentum space. In the trapped atom interferometers discussed here the atom waves remain confined and are separated by moving the potential wells apart.

Important advantages of confined atom interferometers are manifold. The confinement can support the atoms against gravity, offering potentially unlimited experiment times with advantages for precision experiments. The location of the atom wave can be known precisely. This is essential in experiments studying atom-surface interactions like the Casimir potential, or for studying spatially varying fields or interactions with small objects that are coupled to the atoms via an evanescent wave. If a BEC is confined, the large scale coherence allows new ways to measure the relative phase of two condensates using a small sample of the atoms. Additionally, confined atom interferometers, especially those using atom chips, can be small and portable.

Confined atom experiments differ qualitatively from the many experiments that have been carried out using BECs as a bright source of cold atoms propagating in free space (Torii et al., 2000; Gupta et al., 2002). In those the physics is dominated by single-particle dynamics and does not exploit the particular coherence properties of BECs. In the interferometers described here, the intrinsic properties of the BEC allow novel measurements, and create new problems to be overcome.

Confined atom interferometers naturally operate with significant density to achieve the advantages of large signals, from which several disadvantages follow. First, the matter wave optics becomes nonlinear. The atom-atom interactions lead to a mean field potential (the chemical potential in a BEC) that can cause a relative frequency shift between atoms in the two wells. In addition, the potential wells have to be controlled very accurately in stiffness and depth, to prevent additional sources of systematic frequency shifts. (In waveguide interferometers where the atoms are confined only in two directions, any residual potential roughness gives additional problems.)

Splitting a condensate coherently produces a state whose relative phase is specified at the expense of a superposition of number states with different relative populations because of the (approximate) number-phase uncertainty relation. Knowing the relative phase of two condensates requires an uncertainty in the relative number of atoms in each well, even though the total number may be certain. The wave function in each well is there- fore a coherent superposition of states with different relative mean field interactions (different relative chemical potentials) and therefore evolve at different rates. The resulting dephasing limits the coherence time to less than $50 \mathrm{~ms}$ for a typical million-atom BEC (with diluteness parameter $n a^{3} \approx 10^{-4}$ ).

In addition, one has to carefully consider the collective excitations of the condensate (e.g., sound or shape oscillations) which may arise if the potential changes too suddenly. This can be overcome by applying techniques from coherent control, as shown by Hohenester et al. (2007) and Grond et al. (2009).

Recombining the split double well into a single trap allows in principle the readout of the relative phase as a relative population difference between the ground state and first excited state (Hinds et al., 2001; Andersson et al., 2002). In the recombination, the nonlinear interactions lead to creation of (fast moving) solitons. These can enhance the sensitivity (Negretti and Henkel, 2004) of phase measurements, but are much harder to control. Consequently, the experiments recombine the split waves by releasing them from the trap, then free expansion reduces the nonlinearity and facilitates the overlap.

Confined atom interferometers have so far come in two types: BECs confined to waveguides (i.e., in two dimensions) which are described next, and those confined in traps (i.e., in all three dimensions) using focused light beams (Sec. III.F.2) and magnetic fields generated by atom chips (Sec. III.F.3). Finally in the last section we describe an example where it is possible to establish and read out the relative phase of two condensates that do not overlap during the entire process and discuss whether this can be seen as a type of interferometry involving two classical objects.

\section{Interference with guided atoms}

Given the existence of optical interferometers using fiber optical wave guides, and the success in confining and guiding ultracold atoms, it is natural to consider similar designs for atoms. While preliminary theoretical study shows that special designs should allow multimode interferometers (Andersson et al., 2002), no interferometer devices involving atom waveguide beam splitters have been demonstrated.

The first waveguide atom interferometer, by Wang et al. (2005) (see Fig. 32) and improved by Garcia et al. (2006), was designed to test coherent propagation in atom waveguides, not waveguide beam splitters. It was a familiar three-grating interferometer in which pulsed light gratings split and recombined a BEC confined in a weakly confining (magnetic) guide along the axis. The $\mathrm{BEC}$ is split at $t=0$ into two momentum components $\pm 2 \hbar k_{L}$ using a double pulse of a standing light wave. A Bragg scattering pulse at $t=T / 2$ then reverses the momentum of the atoms and the wave packets propagate back. At $t=T$ the split wave packets overlap and a third recombining double pulse completes the interferometer. The output port is given by the momentum of the atoms as detected by imaging (typically $10 \mathrm{~ms}$ ) after release 


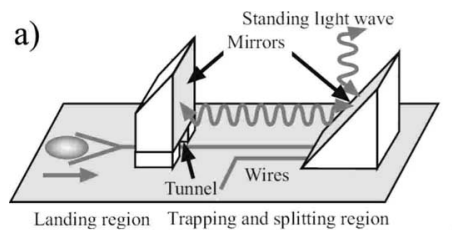

b) 1

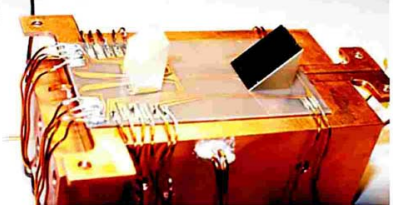

c)

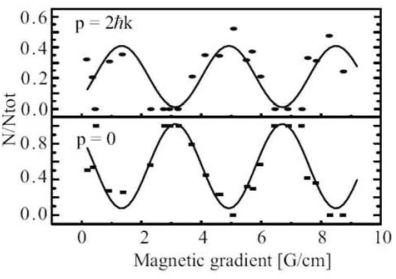

FIG. 32. (Color online) Michelson atom interferometer. (a) Schematic drawing of the atom chip (not to scale). The prismshaped mirrors are integrated with microfabricated wires on an aluminum nitride substrate. The dimensions of the whole chip are $5 \mathrm{~cm} \times 2 \mathrm{~cm}$. (b) Photo of the atom chip on its copper holder. (c) Interference fringes after 1-ms propagation time in the waveguide with the magnetic gradient turned on for $500 \mu \mathrm{s}$ while the average separation of clouds is $8.82 \mu \mathrm{m}$. Adapted from Wang et al., 2005.

from the guide. To apply a phase shift between the two arms of the interferometer, a magnetic field gradient was turned on for a short $(500 \mu \mathrm{s})$ time while the atom clouds were separated. In the original experiment (Wang et al., 2005) the propagation time in the interferometer was varied from $T=1$ to $10 \mathrm{~ms}$. The contrast of the fringes was as high as $100 \%$ for $T=1 \mathrm{~ms}$, but droped to $20 \%$ for $T=10 \mathrm{~ms}$. The degradation of the contrast is mainly due to the nonlinear term coming from the interaction between atoms. By reducing the transverse confinement and consequently the nonlinear interaction Garcia et al. (2006) reached much longer coherent propagation up to $180 \mu \mathrm{m}$ and times up to $50 \mathrm{~ms}$.

There is ample optical precedent for waveguide interferometers using two-dimensional confinement since there is wide application of optical fiber interferometers both scientifically and commercially. On the other hand, interferometry with three-dimensionally trapped atoms has no precedent in light optics. ${ }^{11}$

\section{Coherent splitting in a double well}

Three-dimensional trapped atom interferometers are a qualitatively new type of interferometer without precedent in optics since it is not possible to trap photons, move the trap around, and then somehow recombine the photons. A trapped atom interferometer does just that.

Coherent splitting of the wave function by slowly deforming a single trap into a double well is the generic trapped atom beam splitter, achieving physical separation of two wave-function components that start with the same phase. When the two wells are well-separated,

\footnotetext{
${ }^{11}$ One could argue that a Fabry-Perot is an (imperfect) trap for photons and that the LIGO interferometer which uses Fabry-Perot interferometers nested in a Michelson interferometer is not far from this precedent.
}

a)
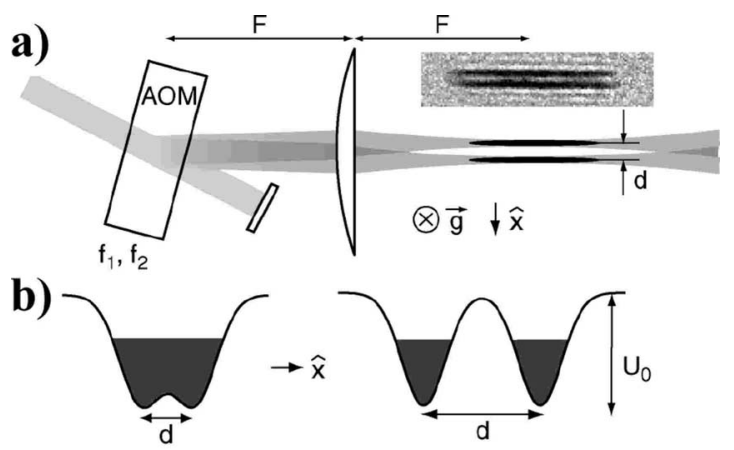

c)
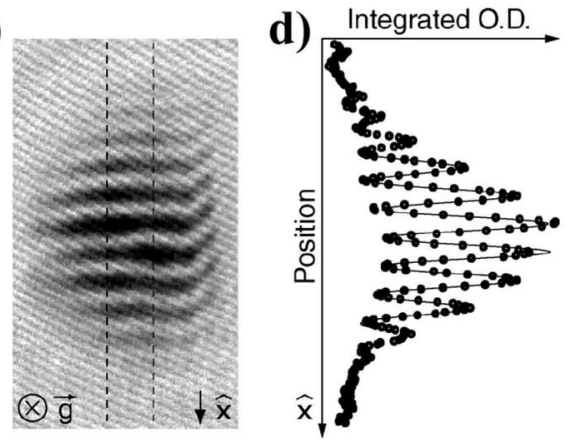

FIG. 33. A double-well interferometer. Top: Optical doublewell potential. (a) Schematic diagram of the optical setup for the double-well potential. The inset shows an absorption image of two well-separated condensates in the double-well potential (the field of view is $70 \times 300 \mu \mathrm{m}$ ). (b) Energy diagram, including the atomic mean field, for the initial single-well trap with $d=6 \mu \mathrm{m}$ and for the final double-well trap with $d$ $=13 \mu \mathrm{m}\left(U_{0}=5 \mathrm{kHz}\right.$, atomic mean field energy $\sim 3 \mathrm{kHz}$, potential "barrier"). (c) Absorption image of fringes created by condensates released from the double-well potential immediately after splitting (30 ms of ballistic expansion, field of view 600 $\times 350 \mu \mathrm{m}$ ). (c) Density profile obtained by integrating the absorption signal between the dashed lines. Adapted from Shin et al., 2004.

an interaction may be applied to either. Finally the split atoms in the two wells are recombined to observe the interference.

Such coherent splitting was first demonstrated by Shin et al. (2004) (see Fig. 33) who split a BEC by deforming an optical single-well potential into a double-well potential. A BEC was first loaded into the single trap and allowed $15 \mathrm{~s}$ to damp its excitations. The splitting was done over $5 \mathrm{~ms}$, slow enough compared to a $600-\mathrm{Hz}$ transverse oscillation frequency in the trap not to excite substantial transverse excitation of the two new condensates, but not slow enough that the mean field interaction would cause the atom to divide exactly evenly between the two wells (with exactly $N / 2$ on each side there would be no number uncertainty and hence the relative phase would have been indeterminate).

The interferometer was completed by releasing the trapped separated BECs and determining their relative phase from the resulting fringes. Releasing the condensates dramatically lowers the mean field interaction prior to overlap, hence averting problems arising from the nonlinearity of atom optics. Another advantage is 
that overlapping two BECs produces high-contrast fringes, enabling an accurate determination of the phase from each "shot" of the interferometer.

Observing the fringes in repeated experiments, starting with fresh condensates each time, addressed the key question: Is the relative phase between the split condensates random or consistent from shot to shot? There had been some theoretical controversy on this subject. The fringes observed when the load, split, and immediate release sequence was repeated were in the same place, showing that the relative phase between the two condensates was consistent, i.e., that it can be controlled deterministically. It was also shown that the phase evolved coherently for up to $5 \mathrm{~ms}$.

The condensates were separated by $13 \mu \mathrm{m}$ in these experiments, and the single atom tunneling rate between the two wells was estimated to be $5 \times 10^{-4} \mathrm{~s}^{-1}$, sufficient to uncouple the BECs in separated wells and let their phases evolve independently. It was verified that each condensate evolved phase independently and was phase shifted as expected by a local Stark shift.

This experiment showed definitively that splitting the well led to BECs with a common phase, introduced a new method to determine the phase that was not affected by mean field interactions, and showed that coherence could be maintained for several oscillation periods of transverse condensate motion.

\section{Interferometry on atom chips}

The combination of well-established tools for atom cooling and manipulation with state-of-the-art microfabrication technology has led to the development of atom chips (Folman et al., 2002). Atoms are manipulated by electric, magnetic, and optical fields created by microfabricated structures containing conductors designed to produce the desired magnetic and electric fields. Technologically, atom chip based atom interferometers promise to be relatively inexpensive and presumably are relatively robust. Atom chips have been demonstrated to be capable of quickly creating BECs and also of complex manipulation of ultracold atoms on a microscale. We trace here the development of techniques to coherently split the condensate and perform atom interferometry.

Many basic interferometer designs and beam splitters on an atom chip were conceived and tested (Folman et al., 2002). Most of them rely on splitting a magnetic potential in multiwire geometry. The first experiments demonstrating splitting, but not coherence, were carried out in Innsbruck (1996-1999) with splitting a guide with a Y-shaped wire (Denschlag et al., 1999; Cassettari, Chenet, et al., 2000; Cassettari, Hessmo, et al., 2000) and a trap with a two-wire configuration (Folman et al., 2002).

At MIT interference with random phase using such a two wire setup was observed by Shin et al. (2005). Simultaneously the first coherent splitting of trapped micromanipulated atoms on an atom chip was achieved by Schumm et al. (2005) at Heidelberg, using radiofrequency induced adiabatic potentials (Zobay and Gar- a)
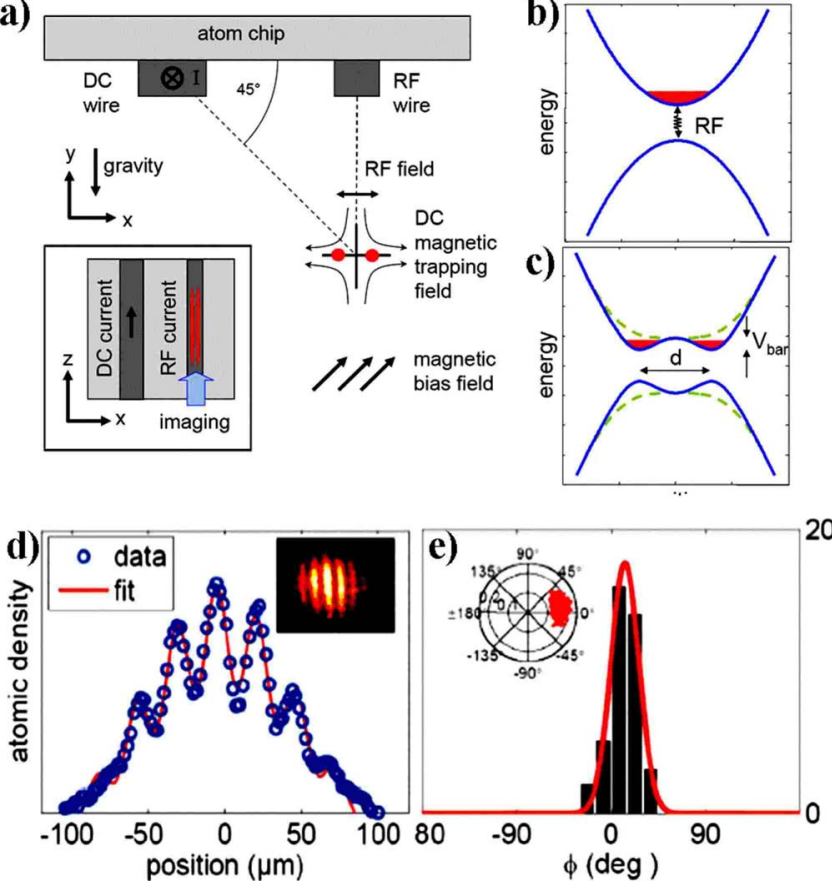

FIG. 34. (Color online) Coherent splitting with an rf induced double well on an atom chip. (a) A wire trap is split by coupling the magnetic substates by rf radiation. To achieve the correct orientation (splitting orthogonal to gravity) the trap is rotated and placed directly over the rf wire. (b),(c) The energy landscape before and after splitting. (d) Interference is observed by switching the trap off and letting the atomic cloud overlap in time of flight. The image integrates over the length of the condensate. (e) Observed distribution of fringe phase and contrast obtained from multiple experiments. Adapted from Schumm et al., 2005.

raway, 2001; Colombe et al., 2004; Lesanovsky, Hofferberth, et al., 2006; Lesanovsky, Schumm, et al., 2006). Analyzing interference patterns formed after combining the two clouds in time-of-flight expansion demonstrated that the splitting is coherent (i.e., phase preserving); see Fig. 34.

The splitting using radio-frequency induced adiabatic potentials as developed in Heidelberg overcomes the disadvantages of the two-wire setup: weak confinement during the splitting and extreme sensitivity to magnetic field fluctuations. The new method allows wellcontrolled splitting over a large range of distancesfrom 2 to $80 \mu \mathrm{m}$-thus accessing the tunneling regime as well as completely isolated sites.

The Heidelberg experiments (Schumm et al., 2005; Hofferberth et al., 2006) are remarkable since they were performed with one-dimensional (1D) BEC (chemical potential $\mu<\hbar \omega_{\perp}$ ), much longer than the phase coherence length. Nevertheless, the interference patterns persist as long as the condensate. All different regimes from physically connected to totally separated $1 \mathrm{D}$ BECs were accessible, and phase locking by coherent tunneling in the intermediate regime could be demonstrated.

With continued progress on these topics, together with techniques for reducing dephasing of interferom- 
eters using BECs, interferometers using confined atoms hold the promise to be employed as highly sensitive devices that will allow exploration of a large variety of physics questions. These range from exploring atomsurface interactions to the intrinsic phase dynamics in complex interacting (low dimensional) quantum systems and the influence of coupling to an external "environment" (decoherence).

\section{FUNDAMENTAL STUDIES}

In this section, we address two questions often asked once one has understood the basic ideas of atom interferometry: Can you make interferometers with any object, people, for example? and Of what use are atom interferometers? We discuss the limits to particle size in Sec. IV.A, experiments that probe the transition from quantum behavior to classical behavior via the process of decoherence in Sec. IV.B, and how the ideas of single particle coherence can be extended in Sec. IV.D. The question of utility is first addressed in Sec. IV.C, where we show that measurable phase shifts arise not only from potential differences, but from things like the Aharanov-Bohm effect and topological transport in general. Then we describe how atom interference can be used to study four different features of many-body systems in Sec. IV.E, and finally address fundamental tests of charge equality for protons and electrons. The order of the sections does not reflect the answers to these questions in sequence, however; rather the first three address single particle questions, Sec. IV.D addresses extensions of coherence first to extended single particles, and then to multiparticle systems, and Sec. IV.E is devoted to describing studies of many-particle systems that reveal many-particle coherence and decoherence processes, or in which atom interference is the tool that enabled the study of their collective properties.

\section{A. Basic questions: How large a particle can interfere?}

When the first atom interferometers were demonstrated, some expressed surprise that "composite" particles would give such high-contrast fringes. These sentiments are in line with the idea that there exists a quantum-classical boundary and that somehow there must be a limit on the number or spacing of internal states (i.e., the "complexity") for particles in an interferometer. Perhaps the mass, the size of a molecule, or the strength of interactions with the environment can limit or eliminate the interference. In this section we investigate the limits to coherent manipulation of the center of mass motion of larger and more complex particles, and point to some open problems. We first consider practical limits set by particle size, grating size, and interactions with the grating, and then move on to more fundamental limits determined by interactions with the surrounding environment.

Experiments with $\mathrm{Na}_{2}$ molecules (Chapman, Ekstrom, et al., 1995) demonstrate that particles with many internal states show interference fringes even if the paths go on opposite sides of a thin conductor. These experiments also confirm what the first atom interferometers showed: interference fringes can be observed when the size of the particle is considerably larger than both its de Broglie wavelength and its coherence length. For example, in the separated beam interferometer with $\mathrm{Na}_{2} \lambda_{\mathrm{dB}}$ $\approx 10 \mathrm{pm}$ and the coherence length $l_{\text {coh }} \approx 100 \mathrm{pm}$ are both much smaller than the size of the molecule $(\sim 400 \mathrm{pm})$. For experiments with $\mathrm{C}_{60}$ or larger molecules the parameters are even more extreme (Clauser, 1997; Arndt et al., 1999, 2001, 2005; Brezger et al., 2003; Hackermuller, Uttenthaler, et al., 2003).

Perhaps more surprising is the observation of fringes in Talbot-Lau interferometers with hot particles like $\mathrm{C}_{60}$, the surprise being that they have a spontaneous emission rate fast enough to emit IR photons during the interference process. But since the maximum separation of the paths in these experiments (about a grating period) is much less than the wavelength of the IR radiation, a few photons of emitted radiation cannot be used to localize the molecule to one path or the other (Hackermuller et al., 2004; Hornberger, 2006). Thus the interference is between two spatially separated paths along which the molecule emitted a photon and changed from internal state $|i\rangle$ to final state $|f\rangle$. Interestingly, IR emission would localize a molecule on one side or the other of a conducting plate, so hot molecule interference would not occur between paths separated by a conductor. ${ }^{12}$ This makes an important point: information left in the environment is sufficient to destroy the coherence; no actual measurement by a macroscopic apparatus is necessary.

Even though a particle's size itself poses no fundamental limit to matter wave interferometry, there are more practical limitations to interferometry with large particles, such as (i) the time required to propagate through an interferometer, (ii) the requirement that the particles fit through the openings on material gratings without undue effects from van der Waals interactions, and (iii) whether laser-based beam splitters can work with particles larger than the laser wavelength.

The time it takes a diffracted particle (with one grating momentum $\hbar G$ ) to move one grating period sets the characteristic time for interference of a particle of mass $m$,

$$
t_{\text {char }}=\frac{d}{\hbar G / m}=\frac{m d^{2}}{h}=\frac{\hbar}{2 E_{G}},
$$

where $E_{G}$ is defined as in Eq. (6). For a grating period $100 \mathrm{~nm}$ and a flight time of $1 \mathrm{~s}$ this limits the mass to $\sim 10^{-17} \mathrm{~g}$, or about $10^{6} \mathrm{Na}$ atoms. Such a cluster would have a size of $\sim 30 \mathrm{~nm}$ and would just fit through the gratings. For the 0.01-s flight times characteristic of current Talbot-Lau interferometers, this limit would be around atomic mass $10^{5}$, about an order of magnitude

\footnotetext{
${ }^{12}$ Of course a separated path interferometer, not a Talbot-Lau interferomter, would be needed for this experiment.
} 
heavier than current practice. Increasing the time by an impractical factor (e.g., to a year, with concomitant inertial stabilization of the gratings) does not improve the mass limit proportionately. The reason is that the grating period has to be increased to accommodate the diameter of the particle (Schmiedmayer et al., 1997; Hegerfeldt and Kohler, 1998, 2000; Schöllkopf et al., 1998) which grows as $m^{1 / 3}$. Thus a year-long interferometer can barely interfere a large bacterium as pointed out by Schmiedmayer et al. (1997).

While this discussion of size or mass limits applies accurately to Talbot-Lau interferometers, the requirements of a separated beam interferometer are several times more stringent. In order to separate the paths the beam must be collimated to better than the diffraction momentum, which requires that the beam (and its transverse coherence length) be several grating periods wide. To separate these wider beams, the particles must propagate for several characteristic times. Even worse, the flux of particles will be dramatically reduced due to the tight collimation. In contrast, Talbot-Lau interferometers have no restriction on their width. Not surprisingly they are the interferometer of choice for demonstrating interference of heavy particles. And even with them, it will be some time before sentient beings can be sent through an interferometer and subsequently asked which path they took.

While Eq. (24) shows that if molecules spend too little time in the interferometer, they will not exhibit quantum interference (Oberthaler, Bernet, et al., 1996); on the other hand, if particles spend too long interacting with mechanical gratings, they will interact with the grating bars, or be diffracted into very high orders. This is because of van der Waals or Casimir-Polder interactions between molecules and the grating bars (Grisenti et al., 1999). To keep half the diffracted molecules in the central $n$ orders requires

$$
\partial V(r) /\left.\partial r\right|_{r=d / 8}<h n v / d^{2},
$$

where $V(r)$ is the atom-surface interaction potential. Equation (25) assumes a grating with an open fraction of $50 \%$ and a grating thickness equal to the grating period (d) (Perreault et al., 2005). Brezger et al. (2003) and Hornberger et al. (2004) discussed how the useful range of molecular velocities for a TLI gets severely restricted for large molecules or small gratings. van der Waals interactions also set a minimum mechanical grating period for Sagnac gyroscopes. For a large Sagnac response factor, one would naturally select small grating periods. However, van der Waals (vdW) interactions cause the uncertainty of a Sagnac rotation sensor to increase if grating periods smaller than $44 \mathrm{~nm}$ are used with $1000 \mathrm{~m} / \mathrm{s} \mathrm{Na}$ atoms. For helium atoms, which have much weaker vdW interactions, the optimum grating period for a rotation sensor is $8 \mathrm{~nm}$, about ten times smaller than current practice. This has been discussed for a MZI by Cronin et al. (2005).

These limitations from grating bars and van der Waals interactions have led to new types of Talbot-Lau interferometers for large molecules where at least one grat- ing is replaced by a light grating: Kapitza-Dirac-TalbotLau interferometer (Brezger et al., 2003; Gerlich et al., 2007; Hornberger et al., 2009). If the particle's size is a large fraction of the wavelength, the light forces will have gradients inside the particle that will excite the collective oscillations of the particle unless the turn on-off time extends over many periods of oscillation. For even larger homogeneous particles the light force averages out to nearly zero. This can be overcome by localizing the interaction [e.g., with a color center (Nairz et al., 2001; Hornberger et al., 2004)] or by making particles with periodic structure on the scale of the wavelength. Nevertheless, the question of how much internal excitation will occur still remains to be answered. Finally, it should be possible to impart lots of momentum with long wavelength photons using multiphoton processes.

\section{B. Decoherence}

Quantum mechanics makes assertions so at odds with everyday experience that the mechanisms by which a quantum mechanical treatment of macroscopic objects reduce to purely classical behavior have long been considered a fascinating topic. Indeed, wrestling with this problem has led many to make radical suggestions for changes in quantum theory itself (e.g., spontaneous projection, pilot wave, etc.) or the nature of reality (many worlds, etc.). Observation of decoherence, and the suppression, avoidance, control, and correction of decoherence mechanisms is an active field made especially topical by the fruits of, and need for, advances in quantum computation and nanotechnology.

Atom interefrometry is based on coherence and therefore is sensitive to interactions that upset this coherence. Relative to neutrons, atoms have large polarizability, magnetic moment, and scattering cross sections and are therefore both more sensitive to, and easy to use as quantitative probes for, decoherence processes. In this section we discuss atom interferometry's historical role in gedanken experiments about quantum uncertainty and its present role in providing an environment in which clean quantitative tests of decoherence is possible.

\section{Interference and "welcher-weg" information}

Perhaps the first general realization about interference fringes was that they can easily be destroyed by interactions that, even in principle, allow one to determine which path an atom took through the interferometer. This is deeply rooted in Bohr's principle of complementarity which forbids simultaneous observation of the wave and particle behaviors. It is best illustrated in the debate between Einstein and Bohr on the question "can one know which path the particle took and still observe the interference of the waves?" (Bohr, 1949; Wooters and Zurek, 1979). Einstein proposed the famous recoiling-slit experiment to measure which path the particle took through a two-path interferometer. In reply Bohr pointed out that the slit itself must also obey the 
laws of quantum mechanics and therefore is subject to the Heisenberg uncertainty principle. He showed quantitatively that if the initial momentum of the slit assembly is known well enough to permit the recoil measurement of which path the particle took, then the initial position of the slit must have been so uncertain that fringes would be unobservable.

According to Feynman, this experiment "has in it the heart of quantum mechanics. In reality it contains the only mystery" (Feynman et al., 1965). (Subsequently Feynman acknowledged that entanglement was another mystery.) In 1960, Feynman proposed a related gedanken experiment in which a perfect light microscope (i.e., one fundamentally limited by Heisenberg uncertainty) is used to determine "which-way" information in a two-slit electron interferometer by analyzing a single scattered photon (Feynman et al., 1965). In Feynman's analysis of this gedanken experiment, electron interference (a manifestly wavelike behavior) is destroyed when the separation of the interfering paths exceeds the wavelength of the probe (i.e., when it is possible to resolve on which path the electron traversed). In fact, the contrast is lost whether or not anyone actually looks with the microscope; the ability in principle to identify the electron's path is enough to destroy the interference pattern. Feynman concludes, "If an apparatus is capable of determining which hole the electron goes through, it cannot be so delicate that it dos not disturb the pattern in an essential way."

More recently a quantitative duality relation was derived by Jaeger et al. (1995) and Englert (1996) to quantify how much "which-path" knowledge $(K)$ can be obtained and how much contrast $(C)$ can be observed at the output of an interferometer,

$$
K^{2}+C^{2} \leqslant 1
$$

It is based on the analysis of a detector that quantifies how well the two paths can be distinguished. The detector could be similar to Feynman's light microscope, as studied theoretically by Stern et al. (1990); Tan and Walls (1993); Tegmark (1993); Steuernagel and Paul (1995); Geotsch and Graham (1996); Holland, Marksteiner, et al. (1996); Wiseman et al. (1997), and examined experimentally by Clauser and Li (1994b); Chapman, Hammond, et al. (1995); Kokorowski et al. (2001); Mei and Weitz (2001). Alternatively the detector could monitor spin polarization or the internal state of atoms as proposed by Scully et al. (1991), discussed by Luis and Sanchez-Soto (1998); Badurek and Rauch (2000); Englert et al. (2000); and examined experimentally by Durr et al. (1998a, 1998b) and Durr and Rempe (2000a). We also note the similarity with many neutron spin-superposition experiments (Bonse and Rauch, 1979; Badurek et al., 1983, 1988; Summhammer et al., 1983).

Modern decoherence theories no longer invoke Bohr's collapse postulate, and they do not rely on the uncertainty principle. Instead they treat quantum systems (such as atoms in an interferometer) as being coupled to their environment (including the which-way detector) together as one combined (open) quantum sys- tem. In this view, the interaction between the observed quantum system and its (quantum) environment is a unitary process that causes entanglement so that the state of the observed quantum system becomes correlated with the quantum state of the environment. Then a measurement made on the environment allows inferences on the quantum system. For example, if a photon in the environment allows an inference of which path the atom took, then a trace over the environment would reduce the coherence remaining in the atom density matrix, even if the coupling interaction were now turned off. For more details we refer the reader to articles by Joos and Zeh (1985); Zurek (1991, 2003); Tan and Walls (1993); Tegmark (1993), and books by Wheeler and Zurek (1983) and Giulini et al. (1996).

Since atoms couple strongly to electromagnetic fields in a well-understood way, atom interferometers provide ideal tools for studying decoherence.

\section{Internal state marking}

The simplest way of measuring an atom's "path" through the interferometer is by marking it with an internal state of the atom. This is analogous to an interferometer for light where the polarization is rotated in one arm. Measuring the internal state of the atom then determines which path it took, and consequently destroys the interference.

For example, Durr et al. (1998a, 1998b) studied the complementary nature of fringe contrast and path information using atoms prepared in a superposition of internal states before they pass through an interferometer for their external (center of mass) states. The interferometer was based on Bragg diffraction gratings that affect the internal states differently so that the interferometer paths became correlated with internal states. This caused a controllable amount of contrast loss, based on how well the internal states labeled which path the atom took.

These experiments are similar to earlier neutron interferometer experiments where loss of interference caused by correlations between spin polarization and interferometer path was studied (Badurek et al., 1983). In both the atom and neutron experiments the coherence can be retrieved (Summhammer et al., 1983; Durr and Rempe, $2000 \mathrm{a}, 2000 \mathrm{~b})$ by projecting the internal state vector onto a measurement basis that does not allow one to distinguish the encoded internal states. The path information is thereby erased and the full interference contrast regained. This is a nice demonstration that interference will be lost if the internal states contain which-path information; the loss of interference occurs without invoking any coupling to an external environment.

To substantiate that there is no coupling to the environment, note that the transitions to prepare the internal state label are driven with microwave fields that are in coherent states with large photon number uncertainty, and hence one cannot use a measurement of the microwave field itself to get information about whether the atom absorbed a single photon on the labeled path. 
Thus no information about the internal state is transferred to the environment. The coherence is not really gone, it is hidden behind the choice of what to measure (interference or path). One can easily get it back by rotating the basis for the measurement, so that the "which path information" is erased, as done in experiments by Badurek et al. (1983) and Durr and Rempe (2000a, 2000b).

This is different from the decoherence described by the recoiling slit or Feynman's microscope discussed above. There one has to look into the environment to get the coherence back. One has to find the other part of the entangled state.

\section{Coupling to an environment}

We now discuss situations in which the interferometer loses coherence because of coupling to the environment. It is closely related to modern theories of decoherence as will become obvious. As an example, consider that the initial state involves an atom traversing an interferometer and a well-collimated photon incident on the atom; then the final state may involve an atom at the detector and a photon in the environment traveling toward infinity. This is a prototypical example of an interferometer that becomes entangled with an external environment or particle. The interaction and its strength is well-known, but the final state is unknown.

\section{a. Decoherence in diffraction}

Several experiments have demonstrated decoherence due to spontaneous emission of light quanta. Gould et al. (1991); Pfau et al. (1994); Keller et al. (2000) used atom diffraction patterns caused by diffraction from a grating to observe how the spatial coherence of an atom beam gets reduced by spontaneous emission of a photon. A good picture is that the recoil from the spontaneously emitted photon shifts the momentum of each atom randomly, along with its individual diffraction patterns. Since the direction of the final photon is random, these experiments revealed a decrease of contrast of the summed patterns. There was a transition from diffraction to diffusion with increasing probability of spontaneous emission. In a similar spirit, the visibility in the diffraction patterns of fullerenes $\mathrm{C}_{60}$ and $\mathrm{C}_{70}$ has been used to bound the amount of decoherence for the molecule waves caused by emitting thermal photons (Hackermuller et al., 2004).

These experiments can be explained by the random momentum kicks given by the spontaneously emitted photons. Interestingly, the result is the same regardless of the place of the photon emission, as long as it is at or upstream of the grating. Consequently, the effect is the same as if the incident beam had a wider transverse momentum distribution, with associated smaller transverse coherence length.

\section{b. Decoherence in Talbot-Lau interferometer}

In a three-grating Talbot-Lau interferometer, Clauser and $\mathrm{Li}$ (1994b) showed that resonant laser light scattered from atoms in the middle of the interferometer can destroy fringe contrast. This experiment actually detected the fringes by selectively destroying the contrast for different velocity classes that were Doppler shifted into resonance with a laser beam.

More recently, Mei and Weitz (2001) demonstrated that photon scattering in a multiple beam Ramsey interferometer also leads to decoherence for atoms that scatter light. Furthermore, because some of the multiple paths in this experiment cause fringes that are out of phase with the other two-path combinations, it was shown that decoherence of one beam can either increase or decrease the net contrast.

Hackermuller et al. (2004) observed decoherence of internally hot fullerene matter waves caused by emission of radiation in a Talbot-Lau interferometer. This experiment is remarkable, since the emission spectrum of the hot fullerene is very close to thermal radiation, and in that sense looks more like a (mesoscopic) classical particle which "cools" internally by emitting photons during the flight in the TLI.

These experiments can again be explained by the (classical) random momentum kicks given by the spontaneously emitted photons.

\section{c. Photon scattering in an interferometer}

Chapman, Hammond, et al. (1995) studied the loss of coherence in a Mach-Zehnder interferometer when each atom scattered exactly one photon. Loss of contrast was observed which depended on the separation between the two interferometer paths at the point of photon scattering. This is a close realization of Feynman's gedanken experiment, and we discuss it below.

\section{d. Scattering from background gas in an interferometer}

Scattering from a background gas of massive atoms or molecules has also been used to cause a controlled amount of decoherence. Collisional decoherence was observed by Hackermuller, Hornberger, et al. (2003) and Hornberger et al. (2003) with Talbot-Lau atom interferometer, and similar work with a Mach-Zehnder interferometer (Uys et al., 2005) is shown in Fig. 35.

It is interesting to note the difference between the decoherence due to photon scattering and atom scattering. The basic physics processes are very similar, except that the momentum transfer is much larger in the case of the atoms and many of the "collisions" lead to atoms scattered out of the detected beam. Consequently, the loss of contrast in atom collisions is not so bad, but the overall intensity goes down significantly. In addition the atom-atom scattering is a probabilistic process, whereas the photon scattering can be made deterministic [see Chapman, Hammond, et al. (1995)]. Additional theory work on collisional decoherence with massive particles can be found in Kleckner and Ron (2001); Fiete and Heller (2003); Hornberger and Sipe (2003); Hornberger et al. (2004); Vacchini (2004).

Closely related to these atom scattering decoherence experiments are the studies of stochastic or deterministic 

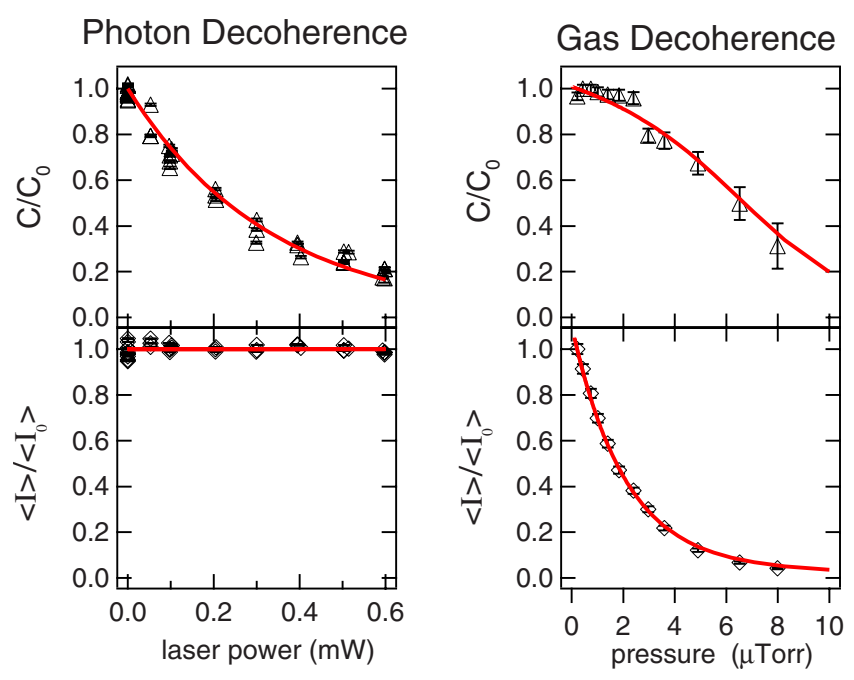

FIG. 35. (Color online) Comparison of decoherence from photon scattering (left) to gas particle scattering (right). Contrast and atom beam intensity as a function of the resonant laser beam power or background gas pressure. The light scattering occurs where the separation $d=0.16 \lambda_{\mathrm{ph}}$, and the gas scattering occurs throughout the interferometer. The theoretical curves come from Eq. (33) for the detected atoms as discussed below. From Uys et al., 2005.

absorption and its effect on coherence in neutron interferometers (Summhammer et al., 1987, 1988; Rauch and Summhammer, 1992; Namiki et al., 1993).

\section{Realization of Feynman's gedanken experiment}

Scattering a single photon from an atom in superposition of two locations is one of the icons of decoherence experiments. It is directly related to Feynman's gedanken experiment discussed above. To realize such an experiment, Chapman, Hammond, et al. (1995) scattered single photons from atoms within a two-path MachZehnder atom interferometer (Fig. 36). Exactly one photon was scattered by adjusting a tightly focused laser beam so that each traversing atom made exactly half a Rabi cycle, exiting the laser beam in the excited state. To achieve this the transit time of the atoms through the excitation laser ( $T_{\text {trans }} \sim 5 \mathrm{~ns}$ ) was much shorter than the lifetime of the excited state $(\tau \sim 16 \mathrm{~ns})$. Translating the laser beam along the interferometer caused excitations at different locations corresponding to different spatial separations of the interfering atom waves.

The experimental results are displayed in Fig. 37. The contrast (which is a direct measure of coherence) decreases smoothly towards zero as the distance between the two paths grows to $d=\lambda / 2$. At this point, the separation between paths is equal to the Heisenberg microscope resolution. The observed contrast recurrences at $d>\lambda / 2$ have their mathematical origin in the Fourier transform of the dipole pattern for spontaneous photon scattering (Tan and Walls, 1993; Steuernagel and Paul, 1995; Geotsch and Graham, 1996; Holland, Marksteiner, et al., 1996). Feynman, who might be surprised at their existence, would be reassured to note that they occur

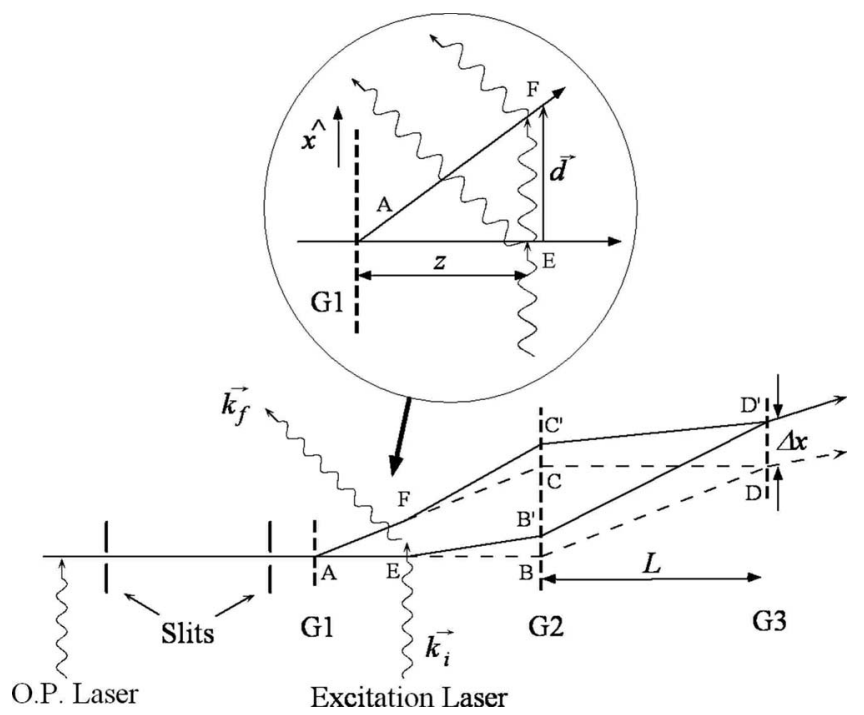

FIG. 36. Schematic for the photon scattering decoherence experiment in Chapman, Hammond, et al. (1995) and Kokorowski et al. (2001). The path separation $d$ and the number of photons scattered per atom can both be controlled. From Chapman, Hammond, et al., 1995.

where the prominent diffraction rings of a perfect light microscope would lead to path ambiguity.

The specific arrangement of the experiment allowed separation of the effects of the (classical) momentum transfer and the entanglement between the atom at two locations and the scattered photon of the related phase shift. As seen in Fig. 36 the average shift of the pattern at the third grating, and its random variation from the recoil of the emitted photons, is much larger than the

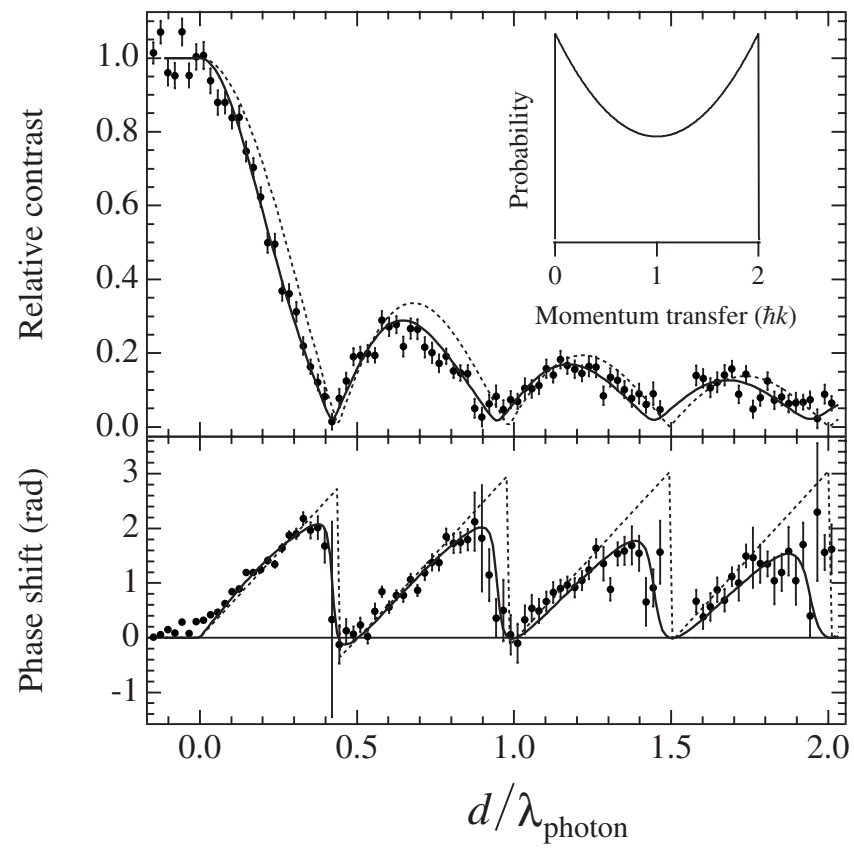

FIG. 37. Contrast as a function of the path separation $d$ at the location of scattering. Each atom scattered nearly exactly one photon in this experiment. From Chapman, Hammond, et al., 1995. 


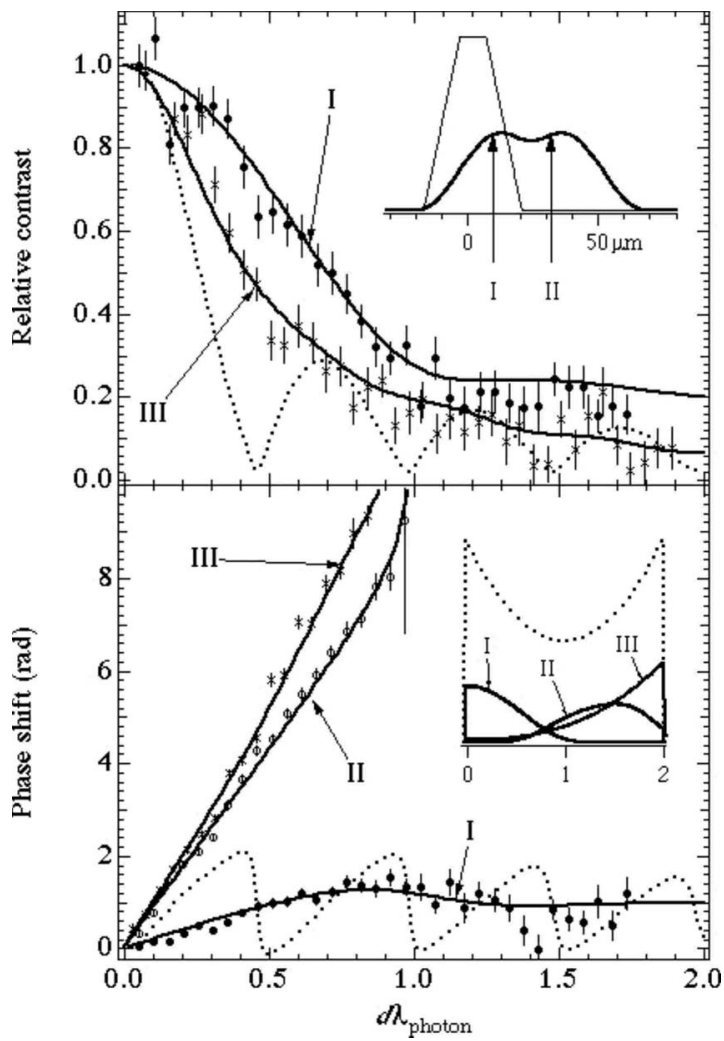

FIG. 38. Relative contrast and phase shift of the interferometer as a function of $d$ for the cases in which atoms are correlated with photons scattered into a limited range of directions. The solid curves are calculated using the known collimator geometry, beam velocity, and momentum recoil distribution and are compared with the uncorrelated case (dashed curves). The upper inset shows atomic beam profiles at the third grating when the laser is off (thin line) and when the laser is on (thick line). The arrows indicate the third grating positions for cases I and II. The lower inset shows the acceptance of the detector for each case, compared to the original distribution (dotted line). From Chapman, Hammond, et al., 1995.

period of the interference pattern at the third grating $(\sim 30 \mu \mathrm{m}$ vs $200 \mathrm{~nm})$. This demonstrates that the momentum recoil by itself cannot explain the loss of contrast (as it can in the diffraction experiments), but the path separation at the point of scattering and the phase shift imprinted by the entanglement in the scattering process must also be taken into account.

The classical recoil shift also allowed a second "recoherence" experiment by allowing the experimenters to infer the momentum of the scattered photon by measuring the atomic recoil. Interference contrast could be regained (Fig. 38) by selecting atoms within a reduced range of momentum transfer. The modern interpretation is that coherence lost to the environment because of entanglement can be regained by learning about the environment. Feynman might say: By restricting the momentum, the microscope could not use the full $4 \pi$ acceptance but only a much smaller numerical aperture. Consequently, the maximum obtainable resolution would be degraded, no which path information obtained, and the interference contrast thereby regained.
This experiment demonstrated the importance of correlations between the recoil momentum and the phase of interference fringes.

These experiments illustrate how the interaction with an environment causes decoherence through entanglement with the states of the environment. If an atom in the two-path interferometer, with the paths separated by $d$, scatters a photon the quantum state evolves into

$$
\begin{aligned}
|\psi\rangle_{i} & =(|x\rangle+|x+d\rangle) \otimes\left|e_{0}\right\rangle \\
& \quad \text { interaction } \\
\stackrel{x}{\longrightarrow}|x\rangle & \left.\otimes e_{x}\right\rangle+|x+d\rangle \otimes\left|e_{x+d}\right\rangle,
\end{aligned}
$$

where $\left|e_{0}\right\rangle$ is the initial wave function of the environment (photon) and $\left|e_{x}\right\rangle$ is the post-interaction wave function of the environment (photon) given an atom at position $x$.

If the environment is now observed to be in state $\left|e_{x}\right\rangle$, the (unnormalized) state of the atom becomes

$$
\left|\psi_{e}\right\rangle=|x\rangle+\beta(d)|x+d\rangle,
$$

where

$$
\beta(d)=\left\langle e_{x} \mid e_{x+d}\right\rangle .
$$

If the two environment states are nearly identical then $|\beta(d)| \approx 1$; very little which-way information is available in the measured state of the environment, and the atom is left in nearly the original superposition. If $|\beta(d)| \ll 1$, significant which-way information about the atom has been left in the environment, and the atom is highly likely, with probability $\left[1+|\beta(d)|^{2}\right]^{-1}$, to be found in state $|x\rangle$.

Whereas Eq. (28) gives the atomic state conditioned on an observation of the environment, we often want to find the final quantum state of the atom when the environment is not observed. This requires averaging over all possible environment states, obtained by taking the trace of the atom and environment density matrix over environment degrees of freedom. Applied to the atom interferometer, this procedure results in a reduction of contrast by a factor $|\beta(d)|$ for every photon scattered, and can be directly applied to describe the results of the Feynman gedanken experiment (Chapman, Hammond, et al., 1995).

Focusing on the which-way information carried away by the scattered photons is not the only way decoherence may be understood. An alternative, but completely equivalent picture involves the phase shift between the two components of the atomic wave function. We switch to this viewpoint using the translation operator for photon momentum states $\left[\hat{T}(\vec{x})=e^{i \hat{k} \cdot \vec{x}}\right]$ to identify that the environment states are related by

$$
\left\langle k_{f} \mid e_{x+d}\right\rangle=\left\langle k_{f}\left|e^{i\left(\hat{k}_{f}-\vec{k}_{i}\right) \cdot \vec{d}}\right| e_{x}\right\rangle,
$$

where the momentum of the absorbed photon $\vec{k}_{i}$ was assumed to be defined by the incident laser beam. Thus if one were to measure the momentum of the scattered photon (to be $\vec{k}_{f}$ ), the atom would then be found in a superposition state with known phase shift between the two components of 


$$
\Delta \phi=\left(\vec{k}_{f}-\vec{k}_{i}\right) \cdot \vec{d} .
$$

Interference fringe patterns for atoms with different recoil momentum kicks will then be slightly out of phase and the ensemble average-the measured interference pattern-will have a reduced contrast. This point of view is useful to calculate

$$
\beta(d)=\left\langle e_{x} \mid e_{x+d}\right\rangle=\int d \vec{k}_{f} e^{i\left(\vec{k}_{f}-\vec{k}_{i}\right) \cdot \vec{d}}\left|\left\langle k_{f} \mid e_{x}\right\rangle\right|^{2} .
$$

This is a scaled Fourier transform of the probability distribution $P(\Delta k)$.

We have discussed two views (which way and dephasing) of the decoherence that accrues when an atom in an interferometer scatters photons. They correspond to two different ways to describe the scattered photon (position basis versus momentum basis). In these two cases, an observer in the environment can determine either which path the atom took or else the phase shift of its fringe pattern. The key point is that when the experimenter is completely ignorant of the state of the scattered photons, whether an apparatus has been set up to measure them or not, the which-path and phase diffusion pictures are equally valid (Stern et al., 1990). Both predict decoherence, i.e., loss of contrast.

Building upon the simple framework of the singlephoton which-way experiment, we can easily derive the effect of continuous atom-light interaction involving many scattered photons. If successive scattering events are independent, the total decoherence function includes one factor of $\beta$ for each scattered photon (with probability $P_{n}$ of scattering $n$ photons). If the separation does not change $(d=$ const), one obtains a simple relation

$$
\beta_{\text {total }}(d)=\sum_{n=0}^{\infty} P_{n} \beta(d)^{n} .
$$

Even at small separations each successive photon scattering found in $\left|e_{x}\right\rangle\left(\left|e_{x+d}\right\rangle\right)$ will reduce by a small factor the probability that the atom is in state $|x+d\rangle(|x\rangle)$ until only one component of the superposition has any remaining amplitude; that is, until "complete" which-path information has been obtained.

This was demonstrated in the experiment by Kokorowski et al. (2001) studying scattering multiple photons from each atom inside the interferometer at a location where the separation is small compared to the light wavelength. The contrast vanishes as information about which path each atom took in the interferometer gradually becomes available in the photon field as a result of multiple scattering events. These experiments are also discussed by Schmiedmayer et al. (1997); Pritchard et al. $(1998,2001)$ and extended to include two separated environments inside the interferometer by Cronin et al. (2003).

Multiple photon scattering results in a Brownian motion of the phase of the atomic superposition and can be analyzed as phase diffusion. It leads again to an exponential decay of contrast as a function of time (i.e., the average number of scattered photons $\bar{n}$ ). Taking the specifics of the photon scattering process one finds, in agreement with the experiment, a Gaussian loss of contrast as a function of the path separation $d$

$$
C / C_{0}=\left\langle e^{i \phi}\right\rangle=e^{-\bar{n}\left(d \cdot \sigma_{k}\right)^{2} / 2},
$$

where $\sigma_{k}$ is the rms spread in momentum per scattered photon.

Contrast loss due to scattering multiple photons makes contact with more formal theories that describe the dynamics of open quantum systems. A modified Heisenberg equation of motion for the density matrix has been derived for various environments by Dekker (1981); Caldeira and Leggett (1983); Joos and Zeh (1985); Gallis and Fleming (1990); Gallis (1993); Tegmark (1993); Omnes (1997); Hornberger et al. (2004). For example, an environment that causes the probability of scattering waves with wavelength $\lambda_{\text {eff }}$ in an infinitesimal time interval $d t$ to be $\Lambda d t$ (where $\Lambda=$ flux $\times$ cross section) makes the master equation

$$
\frac{\partial \rho\left(x, x^{\prime}\right)}{\partial t}=-\frac{i}{\hbar}\left[H, \rho\left(x, x^{\prime}\right)\right]-\frac{\Lambda\left(x-x^{\prime}\right)^{2}}{\lambda_{\text {eff }}^{2}} \rho\left(x, x^{\prime}\right),
$$

where the final term on the right causes a damping of the off-diagonal elements of $\rho$ with a rate expressed by

$$
\rho\left(x, x^{\prime} ; t\right) \approx \rho\left(x, x^{\prime} ; 0\right) e^{-\left[\Lambda\left(x-x^{\prime}\right)^{2} / \lambda_{\text {eff }}^{2} t\right]} .
$$

Here $x-x^{\prime}$ denotes the separation of the superposition states in a general coordinate basis, and the diffusion constant $\Delta=\Lambda / \lambda_{\text {eff }}^{2}$ is also referred to as the localization rate (Joos and Zeh, 1985) or the decoherence rate (Tegmark, 1993). Values of decoherence rates are tabulated in Joos and Zeh (1985); Tegmark (1993); Holland, Marksteiner, et al. (1996) for various systems and scattering environments. Comparing Eqs. (34) and (36) allows one to discuss the localization rate caused by photon scattering for atoms in an interferometer.

\section{Realization of Einstein's recoiling slit experiment}

To implement Bohr's original design of Einstein's recoiling slit gedanken experiment, one needs a very light beam splitter, which shows quantum properties and will allow an experimenter to distinguish the two possible paths taken. In a Ramsey experiment, one would need to be able to distinguish the photons in the microwave or optical field used to change the state in the first interaction region. As discussed above, classical fields cannot do the job. But if the splitting in the first interaction region is induced by a vacuum field, or a single photon field (more generally a field with a definite photon number), then measuring the field will determine if a transition has happened, and consequently infer the path the atom took.

In their seminal experiment Bertet et al. (2001) implemented a Ramsey interferometer with Rydberg atoms where the first interaction zone is a high- $Q$ cavity which allows the superposition between the $|e\rangle$ and $|g\rangle$ states to be created by the interaction with the vacuum field in- 


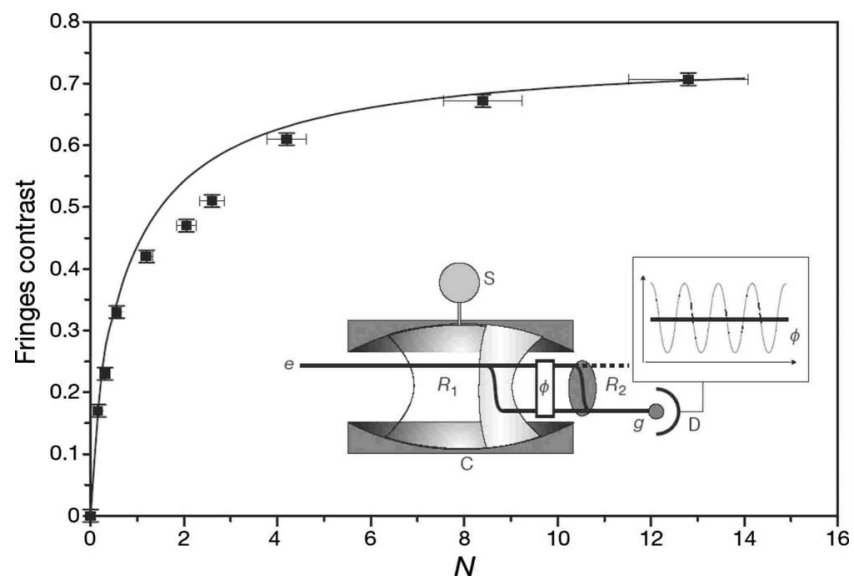

FIG. 39. Fringe contrast as a function of the mean photon number $N$ in $R_{1}$. The points are experimental. The line represents the theoretical variation of the modulus of the beamsplitter final-state scalar product. Adapted from Bertet et al., 2001.

side the cavity. This is the ultimate light beam splitter. After passing the interaction region, the atom-cavity system is in an entangled state described in the $\mid$ atom $\rangle \mid \mathrm{cav}$ ity basis:

$$
|e\rangle|0\rangle \rightarrow(1 / \sqrt{2})\left[e^{i \Phi}|e\rangle|0\rangle+|g\rangle|1\rangle\right],
$$

where $\Phi$ is an phase difference between the two states after the interaction.

With this interaction the information about the state of the atom is left in the cavity field, and no interference contrast is observed when completing the Ramsey interferometer with a classical microwave pulse and state selective detection.

The cavity can also be filled with a very small coherent state $|\alpha\rangle$ with a mean photon number of a few $\bar{n}$ $=|\alpha|^{2}$. The interaction region creates the entangled state

$$
|e\rangle\left|\alpha_{e}\right\rangle \rightarrow(1 / \sqrt{2})\left[e^{i \Phi}|e\rangle\left|\alpha_{e}\right\rangle+|g\rangle\left|\alpha_{g}\right\rangle\right],
$$

with

$$
\begin{aligned}
& \left|\alpha_{e}\right\rangle=\sqrt{2} \sum_{n} C_{n} \cos \left(\Omega \sqrt{n+1} t_{\alpha}\right)|n\rangle, \\
& \left|\alpha_{g}\right\rangle=\sqrt{2} \sum_{n} C_{n} \cos \left(\Omega \sqrt{n+1} t_{\alpha}\right)|n+1\rangle,
\end{aligned}
$$

where $t_{\alpha}$ is an effective atom-cavity interaction time adjusted to give a equal superposition between $|g\rangle$ and $|e\rangle$.

The results of such an experiment are shown in Fig. 39. When employing the lightest beam splitter, that is, the vacuum state with $n=0$, the contrast in the Ramsey interferences vanishes completely. When employing successively stronger coherent states, the beam splitter becomes "heavier" in Bohr's argument, and the coherence returns For $\bar{n}=12.8(|\alpha|=3.5)$ nearly the full interference contrast is regained.

In a second part of their experiment Bertet et al. (2001) employed the same field twice. Once as first interaction region, and again as second interaction region.
In this case even for the vacuum field as a beam splitter no information about the path within the Ramsey interferometer remains, and full contrast was observed. This is an illustration of an unconditional quantum-eraser experiment.

As our understanding of quantum mechanics deepens, and, in particular, as we attempt to exploit quantum mechanics to create more sensitive quantum interferometers, quantum computers, or perfectly secure communication channels based on quantum entanglement, we encounter decoherence as a fundamental limit (Unruh, 1995). Progress relies therefore upon understanding and correcting for decoherence effects. Already our increased understanding of what decoherence means and how to control it has led to the development of quantum error correction codes (Shor, 1995; Calderbank and Shor, 1996; Steane, 1996) and quantum mechanical systems in which certain degrees of freedom are intrinsically decoherence free (Lidar et al., 1998).

\section{Origins of phase shifts}

Phase shifts for interference fringes [see Sec. II.B, Eq. (16)] can be induced by photon scattering as discussed in the previous section [Eq. (31)] or by a variety of other causes such as (i) different potential energy for atoms in each path of the interferometer, (ii) transverse or longitudinal forces on atoms, (iii) inertial displacements such as rotating or accelerating the interferometer platform, and (iv) geometric and topological phase shifts such as the Aharonov-Bohm, Aharonov-Casher, and Berry phases. In the following section we discuss the interrelationship between these types of phase shifts.

\section{Dynamical phase shifts}

Feynman's path-integral formulation (Feynman and Hibbs, 1965; Storey and Cohen-Tannoudji, 1994) relates the wave function at $(\mathbf{x}, t)$ to the wave function at $\left(\mathbf{x}_{0}, t_{0}\right)$ by

$$
\psi(\mathbf{x}, t)=e^{-(i / \hbar)} S_{\Gamma} \psi\left(\mathbf{x}_{0}, t_{0}\right),
$$

where the classical action $S_{\Gamma}$ is defined in terms of the Lagrangian

$$
S_{\Gamma} \equiv \int_{\Gamma} \mathcal{L}[\dot{x}, x] d t
$$

and $\mathcal{L}[\dot{x}, x]$ is the Lagrangian and $\Gamma$ is the classical path from $\left(\mathbf{x}_{0}, t_{0}\right)$ to $(\mathbf{x}, t)$. For potentials that are only a function of position, the wave function acquires a phase shift due to a potential $U(\mathbf{r})$ of

$$
\phi_{\text {int }}=\int\left[\sqrt{\frac{2 m}{\hbar^{2}}[E-U(\mathbf{r})]}-\sqrt{\frac{2 m}{\hbar^{2}} E}\right] d l .
$$

This is analogous to light optics where the wave vector $\mathbf{k}=n(\mathbf{r}) \mathbf{k}_{0}$ depends locally on the index of refraction, and the phase shift due to the index is 


$$
\phi=\int\left(k-k_{0}\right) d l .
$$

To first order in $U / E$ the interaction phase shifter (43) is

$$
\phi_{\mathrm{int}} \approx-\frac{1}{\hbar v} \int_{\Gamma} U(\mathbf{r}) d l,
$$

where $v$ is the particle's velocity.

This brings up the question: When does one measure a quantity described by classical physics like a deflection, and when does one measure a quantity only observable in an interference experiment?. For example, applying a classical force $\vec{F}$ to change a particle's motion is identical to applying a phase gradient to the matter wave. This is because force can be viewed as arising from a potential gradient $[\vec{F}(\mathbf{r})=-\vec{\nabla} U(\mathbf{r})]$, and in the same potential $U(\mathbf{r})$ a propagating matter wave will get a position-dependent phase shift which is exactly the one needed to account for the deflection. If there are two paths through the interferometer, then the fringe phase shift will be given by

$$
\Delta \phi_{\text {int }}=\phi_{\text {int } 1}-\phi_{\text {int } 2} .
$$

Thus in a classical apparatus (as in a moiré deflectometer) or in an interferometer, forces cause a fringe shift that is identical to the classical deflection, which can be observed as an envelope shift (Zeilinger, 1986; Oberthaler, Bernet, et al., 1996).

On the other hand, there are many cases where the fringe shift is different from the envelope shift. A basic example is a constant potential applied to one arm of an interferometer with separated beams. In this case there is no classical deflection, because neither atom (component) acquires a transverse phase gradient. Still, there is a different interaction phase $\phi_{\text {int }}$ for one path through the interferometer because of the potential. For example, one interferometer path may traverse a capacitor such that the gradient in potential energy is along the atomic path.

In this case Longitudinal phase gradients can be caused as atoms enter and exit the interaction region. For example, an attractive potential causes a classical force that first accelerates then decelerates the atom (component); if the potential is confined to one path through the interferometer, then the affected atom component gets displaced ahead of the unperturbed atom component. Furthermore, if the longitudinal displacement between wave-function components exceeds their coherence length, then contrast is lost. We prefer to call this "inhomogeneous broadening" (as opposed to decoherence) because the phase shift is correlated (entangled) with the atom's own longitudinal velocity.

Another interesting case arises when one applies a time-dependent potential to one arm of the interferometer so the atom never sees a gradient in space. An example is the scalar Aharonov-Bohm effect. Then there will be no change in the classical motion and the envelope of the atomic probability distribution will remain stationary as high-contrast fringes (there is no velocity

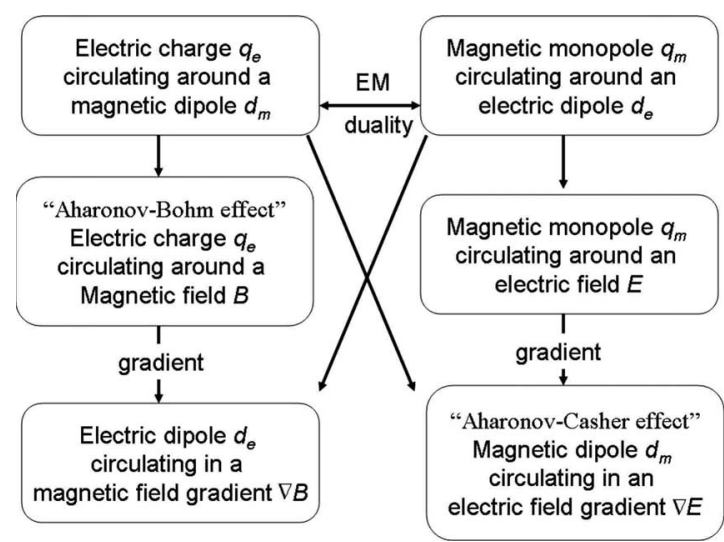

FIG. 40. The Aharonov-Bohm effect, Aharonov-Casher effect, and their electromagnetic duals. Adapted from Panchos, 2003 and Dowling et al., 1999.

dispersion) shift underneath. A similar situation arises when purely topological phases are involved. In these cases the full quantum mechanical properties of an interferometer are in evidence.

\section{Aharonov-Bohm and Aharonov-Casher effects}

We call a phase shift $\Delta \phi_{\text {int }}$ topological if it depends neither on the incident $k$ vector (velocity) of the interfering particle nor on the shape of the particle's path through the interferometer. Topological phases are characteristic of all gauge theories, and are related to a singularity enclosed by the interferometer paths.

The most widely known topological phase was described by Aharonov and Bohm (1959) for a charged particle passing on either side of a solenoid. A related effect was described by Aharonov and Casher (1984) for a magnetic dipole encircling a line of charge. To realize a general framework for the discussion of the quantum interaction between sources and fields we consider Fig. 40. If an electric charge $q_{e}$ circulates around a magnetic dipole $d_{m}$ (or vice versa) then a quantum phase arises (Aharonov and Casher, 1984). Particular configurations of sources can give a variety of contributions. For example, a cylinder filled with aligned magnetic dipoles is equivalent to a solenoid, and creates a homogeneous magnetic field inside the cylinder but zero magnetic field outside. When an electric charge travels around the cylinder it acquires a phase due to the Aharanov-Bohm effect. On the other hand, if a cylinder is filled with electric charges, then a magnetic dipole circulating around it will obtain a quantum phase due to the AharonovCahser effect. This can be generalized to the case of a magnetic dipole moving in the presence of a gradient of electric field.

Employing electromagnetic (EM) duality it is possible to obtain a series of similar phenomena. While the charge dual is the magnetic monopole $q_{m}$, which has never been observed, the dual of the dipoles are welldefined. The interactions between an electric dipole $d_{e}$ and a monopole (or a monopolelike field) have been studied (Casella, 1990; He and McKellar, 1993; Wilkens, 

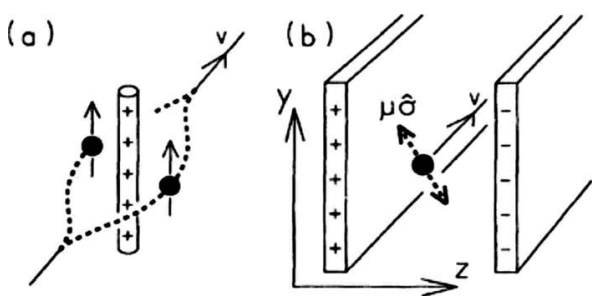

FIG. 41. Aharonov-Casher effect. (a) Geometry of the original measurement using a neutron interferometer, in which the two interfering states encircle a charge and have the same magnetic moments. (b) Geometry used in Sangster et al. (1993). Particles travel in a uniform magnetic field in a coherent superposition of opposite magnetic moments $\pm \mu \hat{\sigma}$. The two states are oppositely shifted by the Aharonov-Cahser phase as they travel through the field. From Sangster et al., 1993.

1994; Dowling et al., 1999; Spavieri, 1999, 2006). This can also be equivalently viewed as the interactions of an electric dipole $d_{e}$ with an inhomogeneous magnetic field. It should be understood that this categorization is not unique or exhaustive, e.g., quadrupole interactions have not been considered.

The Aharonov-Bohm phase shift is

$$
\Delta \phi_{\mathrm{AB}}=\frac{q_{e}}{\hbar} \oint \mathbf{A} \cdot d \mathbf{s},
$$

where $A$ is the vector potential that represents the fields. The Aharonov-Casher effect causes a phase shift

$$
\Delta \phi_{\mathrm{AC}}=\frac{1}{\hbar c^{2}} \oint \mathbf{d}_{\mathbf{m}} \times \mathbf{E} \cdot d \mathbf{r},
$$

where $d_{m}$ is the magnetic dipole.

The Aharonov-Casher effect was observed with neutron interferometers (Cimmino et al., 1989) using the original geometry proposed by Aharonov and Casher (1984), and the phase shift was 2.19 mrad. With TlF molecules the Aharonov-Cahser effect has been observed using a geometry where components of each molecule with different spin states occupy the same center of mass location (Sangster et al., 1993, 1995). This alternative geometry for the Aharonov-Casher effect, in which the magnetic dipoles are placed in a superposition of spin orientations (but not a superposition of center of mass positions), was described by Casella (1990). The two different geometries are summarized in Fig. 41. With molecules possessing a nuclear magnetic moment the phase shift was only $3 \mathrm{mrad}$. Still, this was sufficient to verify the predicted linear dependance on the electric field and independence of particle velocity. Atomic sized magnetic moments were used by Gorlitz et al. (1995) to demonstrate a much larger Aharonov-Caher (AC) phase shift of $300 \mathrm{mrad}$ using $\mathrm{Rb}$ atoms. An AC phase of $150 \mathrm{mrad}$ was observed by Yanagimachi et al. (2002) using $\mathrm{Ca}$ atoms, and related measurements are found in Zeiske et al. (1994, 1995).

The AC phase is a restricted topological phase because although the phase is independent of the speed $|v|$ and the size of the interferometer loop, the phase does depend on whether $\mathbf{d}_{m}$ is perpendicular to both $\mathbf{v}$ and $\mathbf{E}$. Debate over the topological nature of the AC effect has stimulated several discussions, among them Boyer (1987); Aharonov et al. (1988); Zeilinger et al. (1991); Han and Koh (1992); Lee (2001). The similarity between the $\mathrm{AC}$ and $\mathrm{AB}$ effects has also been discussed by Hagen (1990) and Oh et al. (1994). One controversy arose over the question of whether or not a sufficiently large AC phase can lead to decoherence. This position was suggested by Boyer (1987) since the AC effect can be explained in terms of a classical force due to a motioninduced magnetic field in the rest frame of the magnetic dipole. However, as shown by Zeilinger et al. (1991), since the classical force depends on velocity a wavepacket envelope does not get shifted; i.e., $\partial \phi_{\mathrm{AC}} / \partial k_{\mathrm{dB}}$ $=0$. The $\mathrm{AC}$ and $\mathrm{AB}$ effects both shift the phase of the wave function, but do not displace the wave-packet envelope (a common misimpression, e.g., see Figs. 15-7 and 15-8 of Feynman et al. (1965).

The scalar Aharonov-Bohm effect (SAB) for neutral particles is a topological phase that can arise from pulsed magnetic fields interacting with an atomic magnetic dipole. This has been observed by Muller et al. (1995); Shinohara et al. (2002); Aoki et al. (2003) with atoms, and by Allman et al. (1992) and Badurek et al. (1993) with neutrons. It is similar in spirit to the interaction discussed in the original paper (Aharonov and Bohm, 1959) for electrons interacting with the scalar electrostatic potential.

The electromagnetic dual of the AC effect, in which an electric dipole moment moves near a line of magnetic monopoles (an idealized picture of an experiment), was investigated theoretically by Wilkens (1994). The phase shift for polarizable particles moving in both electric and magnetic fields has also been discussed by Anandan (1989, 2000); Shevchenko (1995); Audretsch and Skarzhinsky (1998). Furthermore, in the case that permanent electric dipole moments are used, the electromagnetic dual to the AC effect can be used to settle any controversy regarding how the topological nature of the $\mathrm{AC}$ effect depends on the dipole moment being intrinsic and therefore having quantum fluctuations (Lee, 2001).

\section{Berry phase}

Phase effects resulting from parallel transport associated with adiabatic evolution (Berry phase) can also be topological. Berry (1984) showed that a quantum system in an eigenstate that is slowly transported round a circuit by varying parameters $\mathbf{R}$ in its Hamiltonian $H(\mathbf{R})$ will acquire a geometrical phase factor in addition to the familiar dynamical phase. For example, the Berry phase of a magnetic moment adiabatically following a magnetic field will acquire a phase proportional to the solid angle proscribed by the field during a closed circuit. Berry also interpreted the Aharonov-Bohm effect as a geometrical phase factor.

The Berry phase can be studied with many systems in physics. It has been observed with light in a coiled optical fiber (Chiao and Wu, 1986), neutron interferometers 


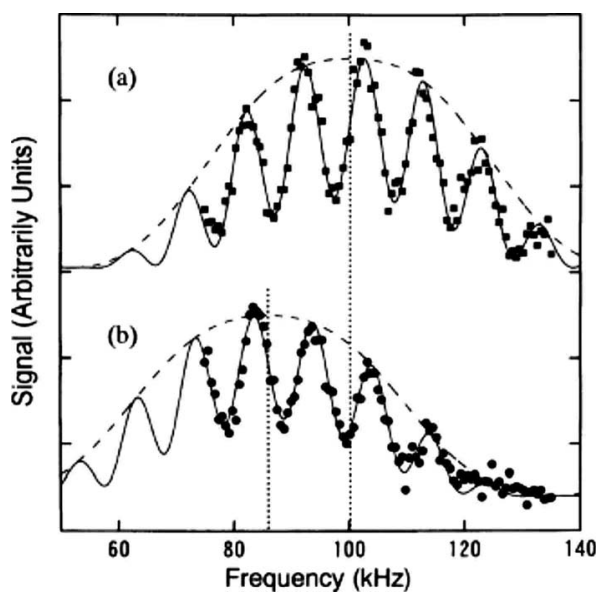

FIG. 42. Ramsey fringes under (a) a constant magnetic field and (b) a rotating magnetic field. The rotating angle is $\pi$. The phase difference is observed at the center frequency of the spectra. From Yasuhara et al., 2005.

(Bitter and Dubbers, 1987), nuclear magnetic resonance experiments (Suter et al., 1987), nuclear quadrupole resonance experiments (Tycko, 1987), and also mesoscopic electronic systems (Yau et al., 2002). Phase shifts due to nonadiabatic circuits (Aharonov and Anandan, 1987), incomplete circuits (Samuel and Bhandari, 1988), particles in mixed states (Sjoqvist et al., 2000), and particles moving relativistically have also been studied theoretically. For an overview on geometric phases, see Wilczek and Shapere (1989) and Anandan et al. (1997).

An observation of a Berry phase in atoms in an interferometer for the polarization states (internal states) has been described by Commins (1991). The first observation of a Berry phase in an external state atom interferometer was accomplished by Miniatura et al. (1992) with a Stern-Gerlach longitudinal interferometer. However, in this experiment the Berry phase was somewhat obscured because the dynamics were not adiabatic. A Berry phase up to $2 \pi$ rad due to an atomic state interacting with a laser field was observed by Webb et al. (1999). This verified the spin dependence of the Berry phase, and realized an "achromatic phase plate for atomic interferometry" as suggested by Reich et al. (1993) and Olshanii (1994). A Berry phase shift for partial cycles using a time-domain atom interferometer was measured by Yasuhara et al. (2005); see Fig. 42.

\section{Inertial displacements}

Atom interferometers are sensitive to acceleration and rotation because the long transit times allow gravity and fictitious forces due to rotation and acceleration to build up significant displacements of the interference pattern, which directly influence the measured phase [introduced in Sec. III, Eq. (18)]. These are discussed in Sec. V on precision measurements.

\section{Extended coherence and BECs}

Bose-Einstein condensates of atomic gasses are very bright sources for atom optics and atom interferometers. Additionally in a gas cooled below $T_{c}$, a significant fraction of atoms are in the condensate, which occupies the lowest translational state of the trap. Typical BECs offer $10^{6}$ atoms confined in a cigar-shaped sample $100 \mu \mathrm{m}$ long and $100 \mu \mathrm{m}$ across, with coherence lengths of the same size, and relative velocities around $0.1 \mathrm{~mm} / \mathrm{s}$. A BEC with its coherence properties (and brightness) constitutes a source analogous to a laser, whereas the traditional thermal atom sources are analogous to thermal sources such as candles or light bulbs in optics.

This ideal source is hindered by the fact that atoms interact which leads to a mean field interactions (chemical potential). A typical condensate would have a density of $10^{14} / \mathrm{cm}^{3}$ with an associated mean field energy of $\sim 1 \mathrm{kHz}(\times h)$, much larger then the ground-state energy of the trap. If the trap is turned off, and the BEC released, this mean field energy dominates the expansion and condensate atoms will separate with several $\mathrm{mm} / \mathrm{s}$ relative velocity regardless of how small the rms velocity was inside the trap. Nevertheless, the resulting momentum spread is still an order of magnitude smaller than the recoil velocity from a resonant photon. It is therefore easy to separate the momentum states differing by a photon momentum in atom interferometers based on BECs as discussed in Sec. III [see, e.g., Fig. 22(c)].

Atom interferometers now offer a powerful tool to study the properties of a Bose-Einstein condensate.

\section{Atom lasers}

Early theoretical studies (Bagnato et al., 1987; Stoof, 1991; Moerdijk and Verhaar, 1994) showed that making a BEC in a trap is easier than making it in free space because the critical density had to be reached only at the bottom of the trap. They showed that the perturbation of the transition temperature and critical number density due to the $s$-wave scattering of atoms was less than $1 \%$, encouraging the then-prevalent view that the condensate is well-described as a blob of very cold atoms. This suggested making a laserlike beam of ultracold atoms simply by extracting atoms from the condensatesuch a beam would have an incredibly low temperature, be almost monochromatic, and have an unprecedented brightness (albeit over a very small cross sectional area with limited total flux).

Early realizations of atom lasers coupled atoms out from a condensate with radio-frequency (rf) pulses, rf chirps, Raman pulses, or weak cw rf radiation (Mewes et al., 1997; Bloch et al., 1999; Hagley et al., 1999). For discussions see also Holland, Burnett, et al. (1996) and Kleppner (1997). The out-coupled atoms have energy given by the out-coupling process plus the mean field energy they gain when emerging from the condensate. In addition, they are accelerated by gravity and any additional potential gradient. The out-coupling frequency can be adjusted as the condensate number changes, to 
account for the changing chemical potential. Moreover, the number of atoms extractable from the condensate is not limited because the condensate can be recharged (Chikkatur et al., 2002) to produce a continuous atom laser beam, although a continuous atom laser is yet to be demonstrated.

In principle, the output from this type of atom laser can have a greater coherence length than the condensate simply because it has the coherence time of the condensate and is traveling. Using a stable BEC as a phase reference could enable feedback to perfectly compensate the changes in chemical potential. So far, however, coherence lengths of atom lasers have not exceeded the size of the condensate.

Phase coherent matter wave amplification, in direct analogy to laser gain, has been demonstrated (Inouye et al., 1999; Kozuma, Suzuki, et al., 1999) and discussed early on by Bordé (1995); Holland, Burnett, et al. (1996); Janicke and Wilkens (1996).

\section{Studies of BEC wave functions}

In the simplest picture of a BEC, all atoms in the condensate occupy the quantum ground state of the trap. This wave function is modified by the mean field interaction of the atoms. As more atoms accumulate in the condensate, their mutual interaction modifies the condensate wave function. For repulsive interactions the condensate wave function broadens at the expense of increased potential energy from the trap in order to minimize the mean field energy. Each atom in the condensate is coherent across the whole condensate and a double-slit experiment in either space or time should show interference fringes.

More sophisticated treatments of atoms cooled below the BEC transition temperature show that they can exist in states called quasicondensates that have short range coherence, but not long range coherence over the whole condensate. Whether BECs have long range coherence was studied in interference experiments on BECs.

Bragg diffraction offers high momentum selectivity. As discussed in Sec. II.C.3, the spread in velocity of atoms that can be diffracted $\left(\sigma_{v}\right)$ is determined by the inverse duration of interaction with the grating, and can be deduced from the time-energy uncertainty principle $\sigma_{v}=2 / \tau G$. Near-resonant standing waves therefore probe a specific velocity class, creating a high-resolution tool for studying BEC velocity distribution. By increasing the interaction time to nearly $1 \mathrm{~ms}$ (Stenger et al., 1999) at MIT achieved a velocity selectivity of $0.1 \mathrm{~mm} / \mathrm{s}$, which allowed us to study the momentum distribution inside the trap and in a released condensate Fig. 43, demonstrating the mean field acceleration. The coherence length was equal to the transverse dimension of the condensate (see Fig. 43, inset).

A similar conclusion was reached independently by Kozuma, Deng, et al. (1999) at NIST using an atom interferometry technique in which brief pulses of outcoupled atoms were generated at two closely spaced times. Each ejected pulse mirrors the condensate itself,

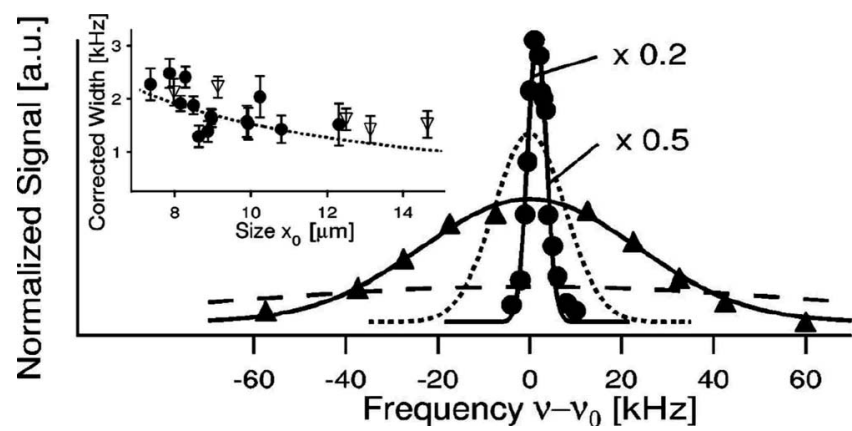

FIG. 43. Bragg resonances for a trapped condensate (circles) and after 3-ms time of flight (triangles). This maps the momentum distribution in the trapped (or expanding) condensate. For comparison, the momentum distributions of the ground state of the trapping potential (dotted curve) and of a $1-\mathrm{mK}$ cold, thermal cloud (dashed curve) are indicated. Inset: Bragg peak widths as a function of condensate size. The plotted Bragg widths have been corrected by subtracting the contribution of the mean field and the finite pulse duration. The dashed curve is based on a prediction for the momentum uncertainty due to the finite size of the condensate and the uncertainty principle. From Stenger et al., 1999.

so when the front of the second overlapped the back of the first the interference observed was indicative of coherence between two spatially separated places in the condensate. The decay of the fringe envelope was as expected for a fully coherent condensate.

Experiments studying the coherence of atom laser beams were carried out by Bloch et al. (2000) in T. Haensch's laboratory in Munich. Two atom laser beams coupled out from different locations of the trap were overlapped to interfere. By changing the separation of the out-coupling locations, and observing the contrast of the interference between the two out-coupled beams they probed the coherence properties of the condensate wave function on length scales approaching $1 \mu \mathrm{m}$ (Fig. 44). Measurement of the temporal coherence of an atom laser has also been used to give an upper limit for temporal phase fluctuations corresponding to $\sim 700 \mathrm{~Hz}$ in the Bose-Einstein condensate (Kohl et al., 2001). The coherence length of an elongated condensate was also studied with matter-wave interferometry by Hugbart et al. (2005).

In a related experiment Anderson and Kasevich (1998) observed interference of atoms from an array of BECs trapped in an optical lattice. The interference between the BECs at different gravitational potential leads to a pulsed atom laser beam. Since many sources contribute, the pulses are much shorter than the separation between them, reminiscent of a mode-locked pulsed laser.

Richard et al. (2003) used momentum spectroscopy (the method of Fig. 43) to study 1D phase fluctuations in Bose-Einstein condensates.

\section{Many-particle coherence in BECs}

The above experiments can all be viewed as looking at single particle coherence. 

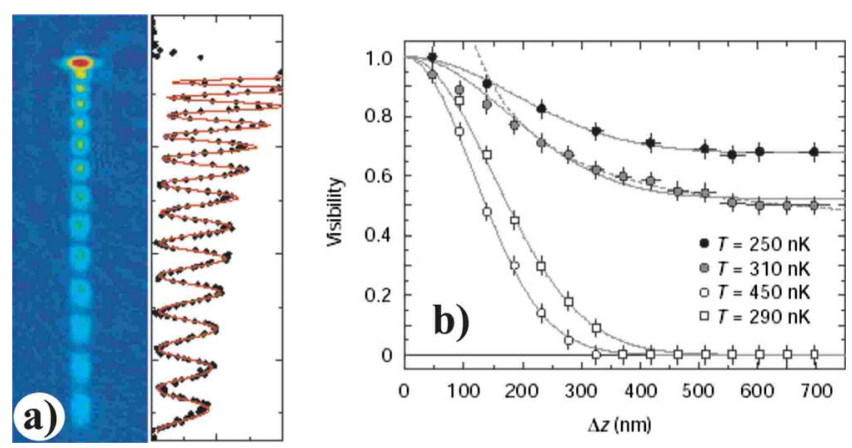

FIG. 44. (Color online) Measurement of coherence length. (a) Interference pattern of matter-wave beams emitted from two spatially separated regions of a trapped Bose gas. (b) Spatial correlation function of a trapped Bose gas as measured by the fringe visibility as a function of slit separation for temperatures above (white circles $T=450 \mathrm{nK}$ and squares $T=290 \mathrm{nK}$ ) and below the critical temperature $T_{c}$ (grey $T=310 \mathrm{nK}$ and black $T=250 \mathrm{nK}$ ), where the visibility decays to a nonzero value due to the long range phase coherence of the $\mathrm{BEC}$. The data points are corrected for the reduction in visibility which is due to the limited resolution of the imaging system. Adapted from Bloch et al., 2000.

BECs have an even more dramatic coherence than the extended condensate wave function. The atoms in the condensate are in one macroscopic state with an order parameter, the phase. Consequently, the phase of one condensate atom is the phase of all. Therefore a condensate also exhibits coherence properties resulting from the interference of different (but indistinguishable) atoms. This gives it coherence properties like a laser: if the phase is determined by measuring some of the atoms, other atoms will have the same phase.

Measuring the phase of condensate atoms requires a coherent and stable reference. Such a reference can be provided by another condensate, or by other atoms from the same condensate. This is in marked contrast to traditional atom interference discussed up to now, where interference is only that of each atom with itself. The BEC experiments using Bragg scattering demonstrate only the spatial coherence of individual atoms in a BEC. We now turn to experiments that show the coherence of different atoms in a BEC.

The existence of a macroscopic wave function with an order parameter means that atoms from different sources can interfere. If an atom from one interferes with an atom from the other, subsequent atom pairs will interfere with the same relative phase and fringes will be built up which reflect the relative phase. This is similar to interference between two independent lasers (Pfleegor and Mandel, 1967; Paul, 1986; Castin and Dalibard, 1997; Kaltenbaek et al., 2006), which also generated controversy prior to its observation.

The first experiment demonstrating this behavior was by Andrews et al. (1997) in the Ketterle group at MIT. To demonstrate that two independent BECs can interfere, two independent condensates were produced in a double-trap potential created by dividing a magnetic trap in half with a focused blue-detuned laser beam. Af-

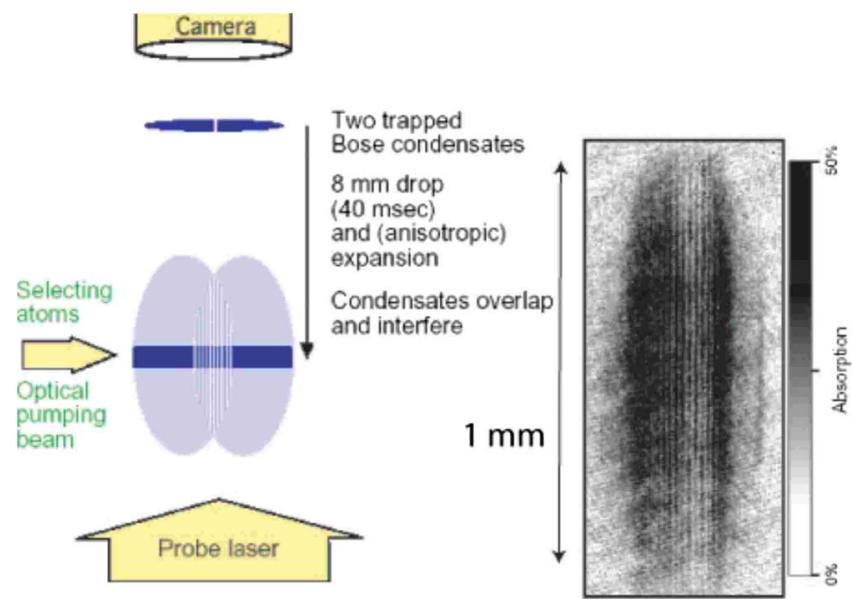

FIG. 45. (Color online) Interference from independent BECs. Left: Schematic setup for the observation of the interference of two independent BECs separated by a barrier created by a blue-detuned laser beam. After switching off the trap, the condensates expand ballistically and overlap. In the overlap region, a high-contrast interference pattern is observed using absorption imaging. Right: Interference pattern of two expanding condensates observed after 40 -ms time of flight. The width of the absorption image is $1.1 \mathrm{~mm}$. The interference fringes have a spacing of $15 \mu \mathrm{m}$ and are conclusive evidence for the multiparticle coherence of Bose-Einstein condensates. Adapted from Andrews et al., 1997, and Durfee and Ketterle, 1998.

ter two BECs were created from separate thermal clouds, the traps were switched off. The atom clouds expanded ballistically and overlapped.

The atomic density in the overlap region was observed directly with absorption imaging, and revealed a high-contrast interference pattern extending over a large region of space (Fig. 45). The interference pattern consisted of straight lines with a spacing of about $15 \mu \mathrm{m}$. This experiment provided direct evidence for first-order coherence and a macroscopic wave function with longrange order in the BEC, and caused some to puzzle over why wave packets expanding radially outwards from two small condensates would produce straight fringes.

In a related atom chip experiment Hofferberth et al. (2006) compared the interference of a coherently split BEC with the interference of two independently created BECs in identical traps (Fig. 46). The coherently split BEC shows a well-defined phase, i.e., the same phase for the fringes each time the experiment is run. In comparison, the independently formed BECs show high-contrast interference patterns but with a completely random phase.

These results are even more surprising than the interference of independent lasers. Theories describing laser sources predict something close to coherent states (for lasers operated well above threshold), which means that each laser beam may be thought of as having a welldefined (if unknown) phase. One cannot, however, assert that the phase of a BEC exists prior to its observation. This is because a BEC at $T=0$ can easily contain a known number of atoms (however many were put in the 
(a)

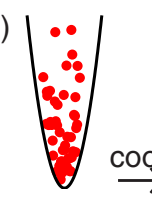

(b)

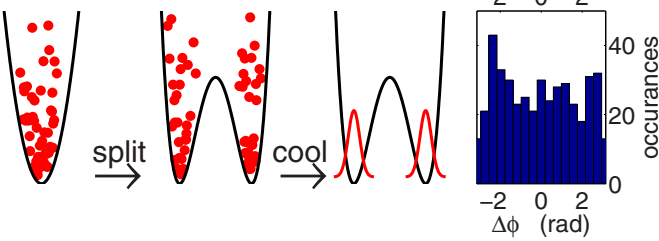

FIG. 46. (Color online) Comparison of independent and coherently split BECs. (a) For the coherent splitting a BEC is produced in the single well, which is then deformed to a double well. A narrow phase distribution is observed for many repetitions of an interference experiment between these two matter waves, showing that there is a deterministic phase evolution during the splitting. (b) To produce two independent BECs, the double well is formed while the atomic sample is thermal. Condensation is then achieved by evaporative cooling in the dressed state potential. The observed relative phase between the two BECs is completely random, as expected for two independent matter waves. Adapted from Hofferberth et al., 2006.

trap), in which case number-phase uncertainty prevents the phase from being specified. So the existence of a well-defined relative phase, and hence fringes in the overlap region seems puzzling.

The resolution to this puzzle is that the phase of the fringes (i.e., the relative phase of the condensates) emerges only as individual atoms are detected in the overlap region (Castin and Dalibard, 1997). Since these atoms cannot be attributed to a particular one of the interfering condensates, an uncertainty develops in the relative number of atoms in the condensates, and in accord with the relative number-phase uncertainty principle, they can have a definite relative phase (even though the total number of atoms in both condensates plus those detected is known). Given that neither the phase of either condensate nor their relative phase existed initially, it should not be surprising that the fringes in each realization of the experiment are observed in a different place. After averaging over many realizations of this experiment, the fringe contrast vanishes because the relative phase of each realization is random.

Even when many independent condensates interfere, spontaneous fringes appear. Hadzibabic et al. (2004) observed high-contrast matter wave interference between 30 Bose-Einstein condensates produced in a largeperiod one-dimensional optical lattice. Interference was studied by releasing the condensates and allowing them to overlap. High-contrast fringes were observed even for independent condensates with uncorrelated phases as shown in Fig. 47. This can be explained the same way as the high-contrast speckles formed by laser light reflecting off a diffuser. However, as in the work with two independent condensates, averaging over many realizations the experiment causes fringe contrast to vanish because the phase is random from shot to shot.
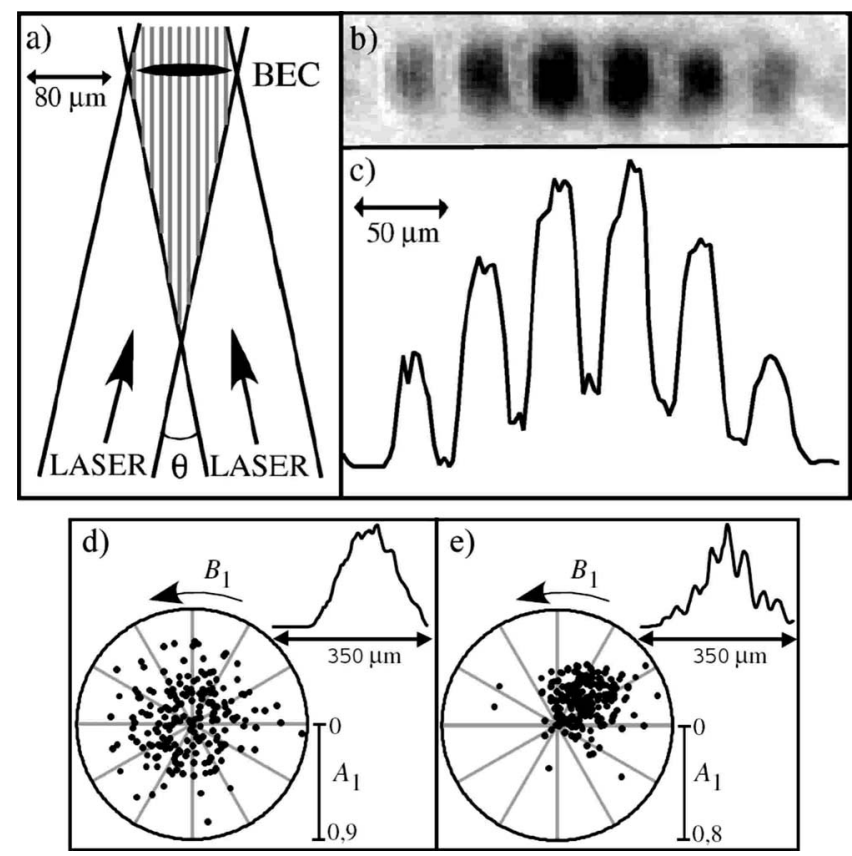

FIG. 47. Interference of 30 Bose-Einstein condensates each containing $\sim 10^{4}$ atoms. (a) A deep 1D optical lattice splits a cigar-shaped condensate into 30 independent BECs. (b) Absorption image of the cloud after $22 \mathrm{~ms}$ of expansion from the lattice. The density distribution shows interference fringes. (c) Axial density profile of the cloud, radially averaged over the central $25 \mu \mathrm{m}$. (d),(e) Polar plots of the fringe amplitudes and phases for 200 images obtained under the same experimental conditions. (d) Phase-uncorrelated condensates. (e) Phase correlated condensates. Insets: Axial density profiles averaged over the 200 images. From Hadzibabic et al., 2004.

\section{Coupling two BECs with light}

Saba et al. (2005) demonstrated a way to make an interferometer using two BECs that are never in direct contact and which are separately trapped at all times. The key is to use stimulated light scattering to continuously sample the relative phase of the two spatially separated BECs. In fact this sampling creates a relative phase between the two condensates which in the beginning had no initial phase relation.

The basis of the measurement is the beating of two atom lasers out coupled from the two condensates by imparting a momentum $\hbar q$. If the relative phase of the condensates is fixed, the total number of out-coupled atoms oscillates sinusoidally with periodicity $h / d$ as $\hbar q$ is scanned ( $d$ is the separation of the condensates). The experimental tool used to impart a precise momentum to atoms is Bragg scattering. Two counterpropagating laser beams with wave vectors $k_{1}, k_{2}$ hit the atoms so that by absorbing a photon from one beam and reemitting it into the other one the atoms acquire recoil momentum $\hbar q=\hbar\left(k_{2}-k_{1}\right)$ (provided that the energy difference between photons matches the atom recoil energy). For each atom out coupled, a photon is transferred from one beam to the counterpropagating one. Therefore all information contained in the stream of out-coupled atoms is also present in the light scattered 
from one beam to the other. Relative phase data were gathered in real time by monitoring the intensity of the weaker of the Bragg laser beams, instead of terminating the experiment to measure the out-coupled atoms using absorption imaging.

Since the relative phase of the condensates can be measured after scattering only a small fraction of atoms out of the condensates, this technique gives a relatively nondestructive measurement of the relative phase. This technique therefore allows one to prepare an initial relative phase (by an initial measurement) of the separated condensates, then to read it out continuously, and thereby to monitor the phase evolution. This way one can realize interferometry between two trapped BoseEinstein condensates without ever splitting or recombining the wave function. The condensates cannot be too far apart, however, as the relative atom number uncertainty cannot arise until atoms out coupled from the first condensate have time to reach the second atom laser beam and create a downstream atom laser whose atoms could have arisen from either condensate. (In fact, when the atom laser beams interfere destructively, the Bragg beams operating on the second condensate effectively capture atoms from the first atom laser beam and insert them in the second condensate.) The necessity for this process to have occurred dictates the temporal delay of the buildup of the light fringes in Fig. 48(a) - it takes about $250 \mu$ s for atoms to make this trip.

This atom interferometer, featuring interference between always-separated ensembles of interacting atoms, is several significant steps away from the prototypical interferometer in which uncorrelated noninteracting individual atoms traverse one at a time. In fact, it resembles a gedanken experiment involving two high- $Q$ $L-C$ circuits resonant with the ac power source in the laboratory. Suppose these are both plugged in to different power outlets for a while, then disconnected. If some time later these are attached to the reference and signal ports of a phase detector, it will read a definite phase. Moreover, this phase will be reproducible shot to shot. If one of the $L-C$ circuits is somehow perturbed, then the phase shift will be systematically modified. Does this situation involving classical $L-C$ circuits constitute an interferometer, or just classical fields interfering?

In fact, it is almost perfectly analogous to the experiment just described, with the roles of matter and electromagnetic waves reversed. The $L-C$ resonant circuits are classical containers containing coherent states of low frequency photons; the light traps are classical containers containing coherent states of atoms. In either case phase shifts can be caused by interactions with the container (squeezing one of the $L-C$ circuit components or light traps), or by interactions with the quantized medium within (e.g., by nonlinear circuit elements added to the $L-C$ circuit or by a magnetic field that interacts with the BEC). The initial coherence is induced by the exchange of photons with the coherent source provided by the power generation station in one case, and by the mutual exchange of atoms in the other. There is a significant interaction among the atoms in the BEC,
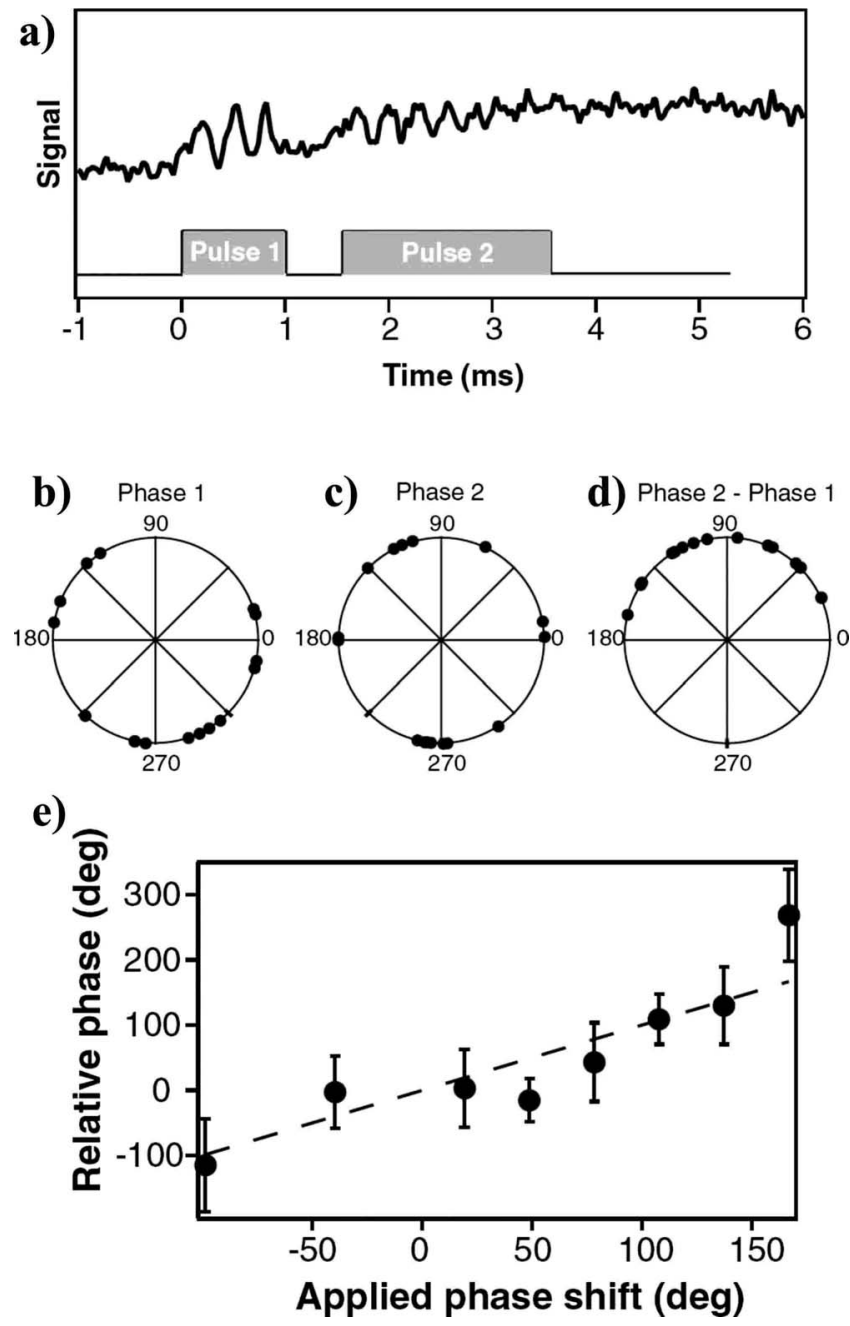

FIG. 48. Preparing a relative phase between two independent BECs with no initial phase relation. (a) The temporal trace of the Bragg beam intensity shown with the pulse sequence. (b) Phase of the oscillations recorded during the first pulse. (c) Phase during the second pulse. (d) Phase difference between (b) and (c). (e) Phase difference between the oscillations in two pulses as a function of the phase shift applied during the evolution time between pulses. Each point is the average of several shots (between 3 and 10). From Saba et al., 2005.

whereas the Kerr effect for $L-C$ circuits is small, but this is not fundamental. Neither the $L-C$ circuits nor the light wells are interfering; both function as classical containers for waves that are phased together. The waves undergo differential interactions, and interfere later to produce a measurable phase shift. Ideally this is solely a measure of the interaction, but in practice small differences between the two containers cause detrimental phase shifts.

\section{E. Studies with and of BECs}

Up to now we have reviewed experiments and theories pertaining to the coherence of BECs. Now we shift perspective and consider them as interesting condensed objects in their own right. Some of the earliest work that 

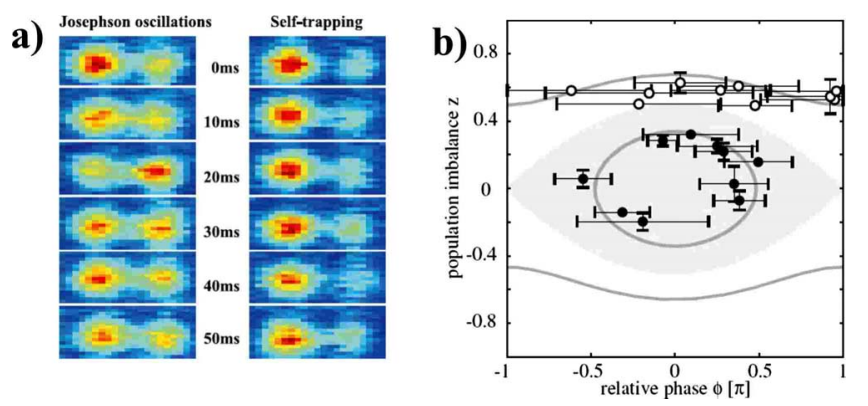

FIG. 49. (Color online) Josephson oscillations. (a) Small population imbalance causes Josephson oscillations, and large population imbalance causes self-trapping. (b) Quantum phase-plane portrait for the bosonic Josephson junction. In the regime of Josephson oscillations the experimental data are represented with filled circles and in the self-trapping regime with open circles. The shaded region indicates the Josephson regime, and the solid lines are obtained by solving the coupled differential with the specific experimental parameters. From Albiez et al., 2005.

showed this was the frequencies of shape oscillations of the condensate. In this section we review experiments made using the techniques and ideas of atom optics and interferometry and that allow one to address other properties of BECs. We review the coupling of two BECs to mimic the physics of Josephson junctions, their intrinsic decoherence, and two experiments that probe their structure.

\section{Josephson oscillations}

As shown by Smerzi et al. (1997), two trapped BECs that are weakly coupled (i.e., by tunneling through the barrier) are represented by a generalization of the equations that apply to a Josephson junction. The analog is that the sine of the phase difference causes a current flow between the traps that changes the number difference (and hence the potential difference that drives the phase change). Given two trapped BECs, by adjusting the tunneling rate (i.e., the coupling strength between the two BECs), Josephson oscillations between two weakly linked Bose-Einstein condensates can be studied.

The experiments of Albiez et al. (2005) demonstrated both the nonlinear generalization of tunneling oscillations in Josephson junctions for small population imbalance $z$ and nonlinear macroscopic quantum self-trapping for large population imbalance. The distinction between the two regimes is apparent in the phase-plane portrait of the dynamical variables $z$ and $\Phi$ as shown in Fig. 49. The successful experimental realization of weakly coupled Bose-Einstein condensates adds a new tool both to condensed matter physics and to quantum optics with interacting matter waves. In particular, we have to realize that the beam splitting (and also the recombination if done at high density) of two BECs must be discussed in terms of the Josephson effect, or possibly its generalization.
A detailed study of the phase noise in the interference patterns allowed Gati and co-workers (Gati, Esteve, et al., 2006; Gati, Folling, et al., 2006; Gati, Hemmerling, et al., 2006) to measure the temperature of the tunnel coupled BECs. Further examples of tunneling were investigated with BECs trapped in optical lattices by Anderson and Kasevich (1998) and Orzel et al. (2001) in M. Kasevich's laboratory and by Cataliotti et al. (2001, 2003) in Florence.

\section{Spontaneous decoherence and number squeezing}

BECs have an intrinsic decoherence due to fluctuations in the number of atoms they contain. If a BEC is prepared in a number (Fock) state, its phase is indeterminate. If its phase is determined, for example, by placing the BEC in a coherent state, then it must be in a superposition of states with different atom number. [For example, a coherent state is a (coherent) superposition of states with different number, with rms variation $\sqrt{N}$.] Since the mean field energy of a trapped BEC increases with $N\left(\sim N^{2 / 5}\right.$ in a harmonic trap), this means the different components have different energy, evolve at a different rate, and get out of phase. The time for this to happen is typically $25-50 \mathrm{~ms}$, severely limiting the accuracy of BEC interferometers.

Even if a BEC interferometer starts with a definite number of atoms in the central well, the $\sqrt{N}$ projection noise at the beam splitter translates into fluctuations of the chemical potential which results in fluctuations in the accumulated phase of the interferometer and, consequently, in a rapid dephasing of the split BEC. The phase diffusion rate can then be estimated by

$$
R_{\phi}=\frac{1}{\hbar} \frac{d \mu}{d N} \Delta N,
$$

where $N$ is the number of atoms in the BEC and $\mu$ its chemical potential. With the chemical potential $\mu$ larger than the trapping frequency $\omega(\mu>\hbar \omega)$ for trapped atoms after typically a few transverse trapping times the phase is random and the coherence is lost. This phase diffusion caused by the interactions between atoms puts stringent limits on the persistence of coherence in a $\mathrm{BEC}$ interferometer.

This interaction-induced dephasing can be reduced in different ways:

- Reduce the effect of interactions by tuning the scattering length with a Feshbach resonance. This may permit setting the scattering length to zero. This requires precise control over the magnetic field, and may limit the number of atoms used in the experiments since the mean field repulsion is proportional to the scattering length and hence the ground-state condensate will no longer be spread out.

- If the method of light scattering described above to measure the phase evolution of the two condensates is applied to two initially number-squeezed condensates (e.g., if a large condensate were separated adia- 
batically), it will add differential number uncertainty only in proportion to how well the phase is determined.

- If the splitting is performed adiabatically, the repulsive interaction itself will tend to equalize the chemical potentials of the splitting condensates. Thus the relative atom number distribution will be reduced if the splitting is performed slowly. This will reduce the relative phase diffusion rate of the initial condensates at the cost of an increased uncertainty in the initial phase, which can be increased to the measurement noise level without penalty. For interferometers using large condensates this can lead to significant increases in their sensitivity and applicability.

In fact, dramatic observations of number squeezing have already been made. Squeezing between atoms trapped in arrays of traps was observed by Orzel et al. (2001). Recently Jo, Shin, et al. (2007) observed a dramatically reduced phase diffusion in a trapped BEC split with an rf splitter on an atom chip, and Esteve et al. (2008) observed number squeezing directly in a BEC split in an optical dipole trap. In a recent theoretical analysis, Grond et al. (2009) showed how to speed up the splitting process by more than an order of magnitude by using optimal coherent techniques.

\section{Structure studies of BEC}

According to theory, a BEC possesses collective modes (e.g., sound waves) due to the interactions of the atoms. In a quantum many-particle description, its dispersion relation has the Bogoliubov form

$$
\nu=\sqrt{\nu_{0}^{2}+2 \nu_{0} \mu / h}
$$

where $\mu=n 4 \pi \hbar^{2} a / m$ is the chemical potential, with $a$ and $m$ denoting the scattering length and the mass, respectively, $n$ is the density of the condensate and $h \nu_{0}$ $=q^{2} / 2 m$ is the free particle dispersion relation (StamperKurn et al., 2001; Ozeri et al., 2005).

In a typical $\mathrm{Rb}$ or $\mathrm{Na}$ condensate, $\mu / h$ is about a $\mathrm{kHz}$, corresponding to speeds of $\sim \mathrm{cm} / \mathrm{s}$ or less. The Bragg spectroscopy discussed previously generates atoms with several times this speed, which therefore have nearly their free-particle dispersion relation (the mean field energy term being negligible). However, by reducing the angle of the Bragg beams from $180^{\circ}$ to much smaller angles, the transferred momentum was correspondingly reduced, and many fewer atoms are liberated from the condensate (i.e., the static structure factor is no longer unity), and the frequency shift relative to a free particle follows Eq. (50). Studies of BEC structure have been given by Steinhauer et al. (2003); Katz et al. (2004), and theory for these measurements has been discussed by Blakie and Ballagh (2000) and Carusotto et al. (2000).

Physics which goes deeper into the properties of degenerate quantum gases and their coherence properties is outside the purview of this review, so we refer the reader to a series of reviews which summarize the status of this evolving field (Dalfovo et al., 1999; Cornell and Wieman 2002; Kasevich, 2002; Ketterle, 2002).

\section{Dynamics of coherence in 1D systems}

Interference allows us to study the dynamics of (de)coherence in degenerate Bose gases. This is especially interesting in the one-dimensional (1D) regime where long-range order is prevented by the ubiquitous phase fluctuations.

In their experiments Hofferberth, Lesanovsky, Fischer, et al. (2007a) coherently split a 1D quasi condensate, characterized by both the temperature $T$ and chemical potential $\mu$ fulfilling $k_{B} T, \mu<h \nu_{\perp}$, along the transverse direction which initializes the system in a mutually phase coherent state, and phase fluctuation patterns of the two individual 1D systems being identical. This highly nonequilibrium state relaxes to equilibrium over time and the evolution of (de)coherence is revealed in local phase shifts leading to increased waviness of the interference pattern (Fig. 50).

If the two parts of the system are completely separated, the equilibrium state consists of two uncorrelated quasicondensates and Hofferberth, Lesanovsky, Fischer, et al. (2007) observed a randomization of the relative phase $\theta(z, t)$ as expressed in the coherence factor $\Psi(t)$ $=(1 / L)\left|\int d z e^{i \theta(z, t)}\right|$. Most interestingly, $\Psi(t)$ decays subexponential $\Psi(t) \propto e^{-\left(t / t_{0}\right)^{2 / 3}}$ as predicted by Burkov et al. (2007) based on a Luttinger liquid approach (Haldane, 1981). Qualitatively similar behavior was observed at MIT (Jo, Choi, et al., 2007) for elongated condensates with $\mu \sim 2 h \nu_{\perp}$ and $T \sim 5 h \nu_{\perp}$.

For finite tunnel coupling between the two systems Hofferberth, Lesanovsky, Fischer, et al. (2007) observed that the final equilibrium state shows a nonrandom phase distribution (Fig. 50, bottom). The phase randomization is counterbalanced by the coherent particle exchange between the two fractions, equivalent to injection locking of two matter wave lasers. The final width of the observed phase spread depends on the strength of the tunnel coupling Gati, Hemmerling, et al. (2006).

\section{Measuring noise by interference}

In many-body systems quantum noise can reveal the nonlocal correlations of underlying many-body states (Altman et al., 2004). Recently it has been suggested that the statistics of the shot-to-shot fluctuations in fringe contrast probe higher-order correlation functions (Gritsev et al., 2006; Polkovnikov et al., 2006).

This rationale was used by Hofferberth, Lesanovsky, Schumm, et al. (2007) in an experiment investigating the statistical properties of interference experiments performed with pairs of independently created onedimensional atomic condensates (Hofferberth et al., 2006). The shot-to-shot variations of interference can then be directly related to the full distribution functions of noise in the system (Polkovnikov et al., 2006). Probing different system sizes they observe the crossover from quantum noise to thermal noise, reflected in a character- 


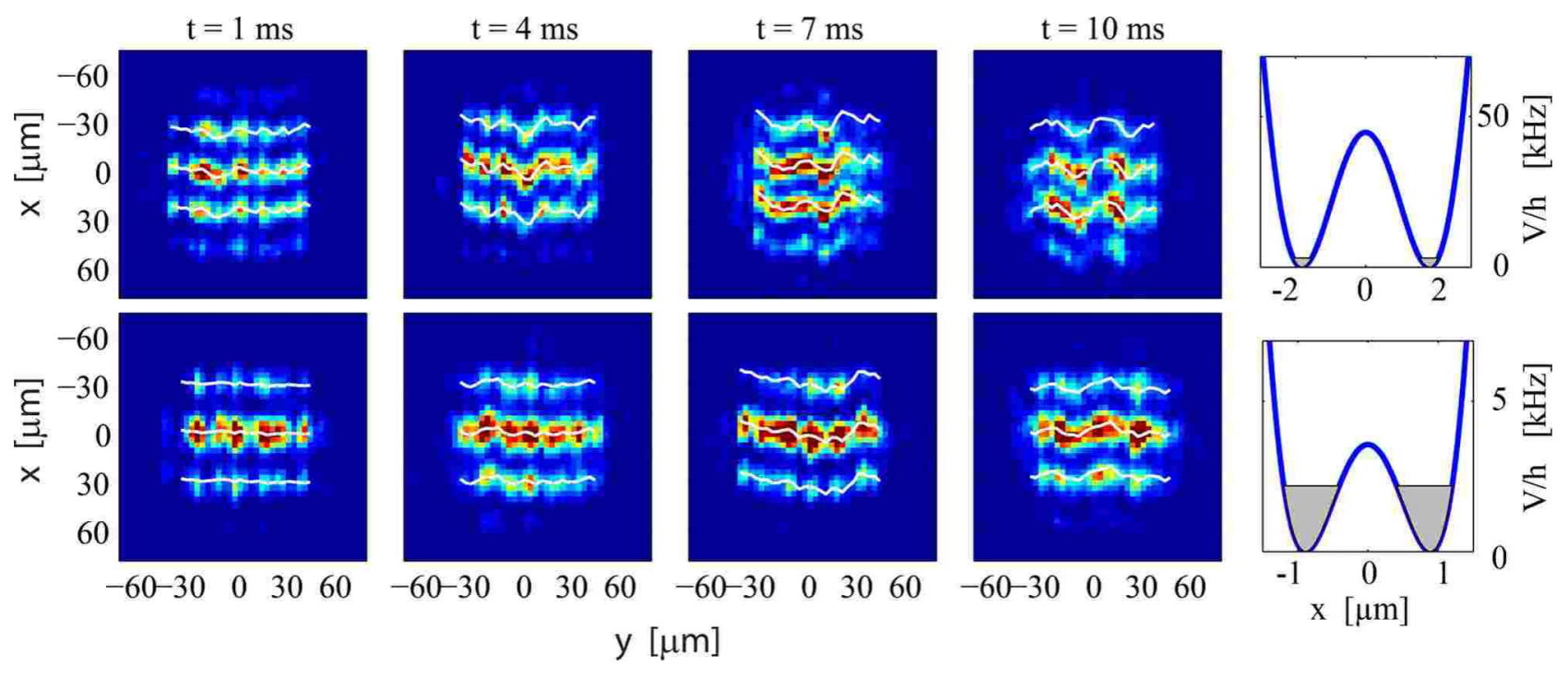

FIG. 50. (Color online) Direct observation of the phase dynamics through interference. Example images of the observed interference patterns for hold times $t=1,4,7,10 \mathrm{~ms}$ (top) in the case of isolated 1D systems and (bottom) for finite tunnel coupling. The different transverse double-well potentials are shown. Adapted from Hofferberth, Lesanovsky, Fischer, et al., 2007.

istic change in the distribution functions from Gumbeltype to Poissonian (see Fig. 51). The results are in excellent agreement with the predictions of Gritsev et al. (2006) based on the Luttinger liquid formalism (Haldane, 1981).

These experiments demonstrate the power of quantum noise analysis in interference patterns as a probe of
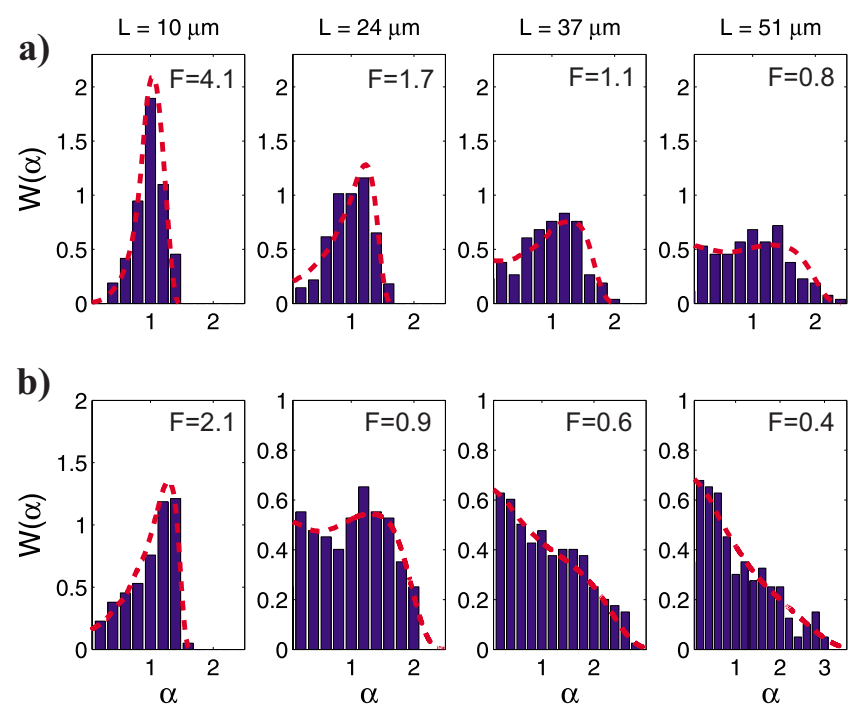

FIG. 51. (Color online) Distribution functions of the measured interference contrasts for different lengths $L$ along the $1 \mathrm{D}$ condensate. (a) The length-dependent normalized interference contrasts $\alpha$ with parameters $\left(n_{1 \mathrm{D}}=60 \mu \mathrm{m}^{-1}, \nu_{\perp}=3.0 \mathrm{kHz}, K\right.$ =46). The curves show the corresponding calculated distributions for $T=30 \mathrm{nK}\left(\xi_{T}=0.9 \mu \mathrm{m}\right)$. (b) Same parameters as in (a), but higher temperature $T=60 \mathrm{nK}$. For both sets Hofferberth, Lesanovsky, Schumm, et al. (2007) observed the predicted change of overall shape of the distribution functions from single-peak Gumbel-type characteristic for quantum noise to Poissonian characteristic for thermal noise. Adapted from Hofferberth, Lesanovsky, Schumm, et al., 2007. correlated systems, and the power of simple ultracold atom systems to exhibit and illustrate fundamental quantum processes relevant in many areas of physics.

\section{Momentum of a photon in a medium}

The momentum of a photon propagating in a medium is a topic fraught with controversy. When an electromagnetic wave enters a medium with index of refraction $n$, its wavelength is reduced, and its wave number is increased by $n$. Thus is seems evident that a single photon in this medium would have momentum $p=n \hbar k_{\text {vac }}$, a conclusion reached by Minkowski $(1908,1910)$ using classical physics. On the other hand, if the photon is considered as a particle, it seems strange that it should increase its momentum when entering a medium in which its speed is reduced. Such a viewpoint is supported by Abraham (1909) who found $p=\hbar k_{\mathrm{vac}} / n$. Resolving these two viewpoints has been cited as one of the challenges of theoretical physics (Peierls, 1991).

When a photon propagating in an atomic gas is absorbed by one of the atoms in the BEC, what is the momentum of the atom after the absorption? This question seems less subject to uncertainty since it can be settled by a measurement; it is also important in precision experiments to measure $h / \mathrm{m}$ as discussed in the next section. For a dilute atomic gas, a third opinion seems justified: a BEC has only a few atoms per cubic wavelength, and no obvious mechanism to transfer momentum to or from the atoms not involved in the absorption-hence the atom will absorb momentum $p$ $=\hbar k_{\text {vac }}$.

In a recent experiment done in a BEC by Campbell et al. (2005), a double pulse Kakpita-Dirac interferometer was used to measure the recoil energy of $\mathrm{Rb}$ atoms in a $\mathrm{BEC}$ for laser frequencies on both sides of the resonance (see Fig. 52). The results showed marked structure 


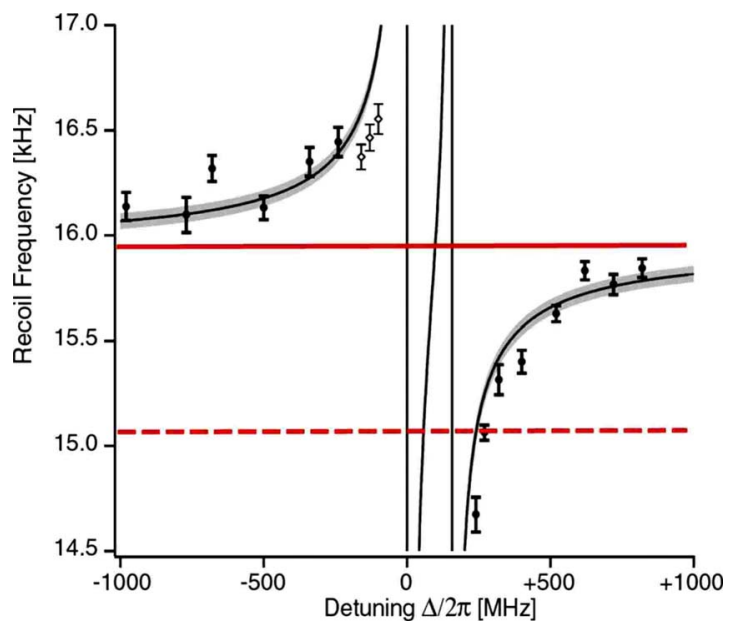

FIG. 52. (Color online) Recoil frequency measured on two sides of resonance. The dashed line shows expected result for free atoms and the solid line corrects for chemical potential assuming $p=\hbar k_{\mathrm{vac}}$. The solid line with error shading shows expectation if $p=n \hbar k_{\mathrm{vac}}$. From Campbell et al., 2005.

near the resonance, consistent with that predicted from the variation of $n$ near the resonance only if $p=n \hbar k_{\mathrm{vac}}$.

With continued progress on these topics, interferometers with BEC hold the promise to be employed as highly sensitive devices that will allow exploration of a large variety of physics questions. These range from atom-surface interactions to the intrinsic phase dynamics in interacting (possibly low dimensional) quantum systems or the influence of the coupling to an external "environment" (decoherence).

\section{F. Testing the charge neutrality of atoms}

The equality of the electrical charges of the electron and proton and the charge neutrality of the neutron are of great significance in the fundamental theory of particles (Chu, 1987; Unnikrishnan and Gillies, 2004). Experimental tests of the electrical neutrality of bulk solid matter and bulk quantities of gas are precise enough at present to state that $\left(q_{p}+q_{e}\right) / e<10^{-21}$ (Dylla and King, 1973; Marinelli and Morpurgo, 1982, 1984). An experiment searching for deflection of a neutron beam has set a similar limit for the electric charge of the neutron $q_{n}$ $<10^{-21}$ (Baumann et al., 1988). Experiments with individual atoms or molecules in a beam have only been able to verify the net electrical charge of $q_{p}+q_{e}$ is less than $10^{-19} e$ (Zorn et al., 1963; Hughes et al., 1988).

A dedicated atom interferometry experiment could detect a phase shift if $\left(q_{p}+q_{e}\right) / e=10^{-22}$. The phase shift would be

$$
\phi=\left(q_{p}+q_{e}\right) Z \mathcal{E} \Delta x L / v \hbar \approx 10^{-4} \mathrm{rad},
$$

where we have assumed $Z=55$ is atomic number, $\mathcal{E}$ $=10 \mathrm{kV} / \mathrm{mm}$ is the applied field, $\Delta x=100 \mu \mathrm{m}$ is the separation of the paths in the interferometer, $L$ is the length of the interaction region, and $v=100 \mathrm{~m} / \mathrm{s}$ is the atomic velocity. Champenois et al. (2001b) and Dehuille
TABLE II. Inertial sensing resolutions demonstrated with atom interferometers.

\begin{tabular}{ll}
\hline \hline Sensor & \multicolumn{1}{c}{ Resolution } \\
\hline Gravimeter $^{\mathrm{a}}$ & $2 \times 10^{-8}(\mathrm{~g}) / \sqrt{\mathrm{Hz}}$ \\
Gravity gradiometer $^{\mathrm{b}}$ & $4 \times 10^{-9}(\mathrm{~g} / \mathrm{m}) / \sqrt{\mathrm{Hz}}$ \\
Gyroscope $^{\mathrm{c}}$ & $6 \times 10^{-10}(\mathrm{rad} / \mathrm{s}) / \sqrt{\mathrm{Hz}}$ \\
\hline
\end{tabular}

${ }^{\mathrm{a}}$ Peters et al. (2001).

${ }^{\mathrm{b}}$ McGuirk et al. (2002).

${ }^{\mathrm{c} G u s t a v s o n}$ et al. (2000).

et al. (2001) studied this. The main difficulty will come from the electric polarizability of atoms which will cause large phase shifts due to field gradients. But because these phase shifts are quadratic in the applied electric field while the proposed effect is linear, these stray phase shifts should mainly limit sensitivity.

\section{PRECISION MEASUREMENTS}

Since their demonstration in 1991, atom interferometers have become precision measurements tools. The advantages of small de Broglie wavelengths, long propagation times, and the narrow frequency response of atoms are responsible for atom interferometers already having made an impact on many fields of fundamental science and engineering. In the present section, we discuss measurements of acceleration, platform rotation, the Molar-Planck constant $\left(N_{A} \times h\right)$, and the fine structure constant $(\alpha)$. Although some measurements of atomic and molecular properties are precision measurements by the standards of those fields, they will all be discussed in Sec. VI.

\section{A. Gravimeters, gryroscopes, and gradiometers}

Inertial sensors based on atom interferometers already perform comparably to the best available sensors based on any technology. At their current levels of resolution summarized in Table II several applications are within reach. In fact, development has begun for commercial sensors and applications using atom interferometers. To explore the precision, resolution, accuracy, response factor, bandwidth, dynamic range, and stability achievable with atom interferometers we begin by looking at the different designs used for gravimeters, gyroscopes, and gravity gradiometers.

Thermal atom beams for rotation, freely falling atoms for acceleration, and two clouds of falling atoms with a common laser beam for the gradiometer have given the best results to date. This is in part because rotation sensors have a response factor (i.e., phase shift per rotation rate) that increases linearly in proportion to the atom's time of flight; but accelerometers have a response factor that increases quadratically with time of flight. Part of the tradeoff is that fast atom beams offer more atoms per second than cold atom sources. Larger interferometers will improve sensitivity and slow atom interferom- 
eters can make compact sensors. In each case, to judge the overall performance one must also look at systematic errors.

Displacements from an inertial reference frame with a constant acceleration $\vec{g}$ and a constant rotation $\vec{\Omega}$ causes a phase shift for a three-grating interferometer

$$
\phi=(\vec{G} \cdot \vec{g}) \tau^{2}+2 \vec{G} \cdot(\vec{\Omega} \times \vec{v}) \tau^{2},
$$

where $\vec{G}$ is the reciprocal-lattice vector of the gratings and $\tau$ is the time of flight for atoms with velocity $\vec{v}$ to travel between gratings (Dresden and Yang, 1979; Anandan, 1981; Malykin, 2000; Bongs et al., 2006; Dubetsky and Kasevich, 2006). Referring to our previous section on the origin of phase shifts, this phase is equivalent to the envelope shift, a classical property. Equation (52) can be derived from the grating phase [introduced in Sec. III, Eq. (18)]

$$
\phi=\vec{G} \cdot\left[\vec{x}_{1}\left(t_{1}\right)-2 \vec{x}_{2}\left(t_{2}\right)+\vec{x}_{3}\left(t_{3}\right)\right],
$$

where $x_{i}$ is the transverse position of the $i$ th grating (with respect to an inertial frame) at time $t_{i}$ (when the atoms interact with the grating).

Rotation about the center grating in a space-domain interferometer causes a phase shift

$$
\phi_{\text {atom }}=2 \Omega G L \tau=4 \pi \Omega m A / h,
$$

where $L$ is the separation between gratings, $\tau$ is the time of flight between gratings, and $A$ is the area enclosed by the interferometer paths. For an optical interferometer,

$$
\phi_{\text {light }}=2 \Omega G L^{2} / c=4 \pi \Omega A / \lambda_{\mathrm{ph}} c .
$$

The ratio of phase sifts for a given rotation rate $(\Omega)$, assuming equivalent interferometer areas $(A)$, is

$$
\frac{\phi_{\text {atom }}}{\phi_{\text {light }}}=\frac{m c^{2}}{\hbar \omega}=\frac{\lambda_{\mathrm{ph}}}{\lambda_{\mathrm{dB}}} \frac{c}{2} \approx 10^{10} .
$$

This ratio shows that atom interferometers have a large Sagnac response factor compared to optical interferometers.

However, to really gain this large increase in resolution (at the expense of bandwidth $v / c$ ) both the enclosed area and the count rate of the two types of interferometers must be equal. But a fiber optic ring gyro can easily have an enclosed area of $A=10^{3} \mathrm{~m}^{2}$ and still have a much better bandwidth compared with the largest atom interferometers that have $A=10^{-4} \mathrm{~m}^{2}$. So the response factor is only a few orders larger for today's atom interferometers. Furthermore, while the count rate for an optical watt of power is on the order of $10^{19}$ photons/s, typical atom interferometers offer only $10^{7}$ atoms $/ \mathrm{s}$.

For acceleration, one can see from Eq. (52) that

$$
\phi_{\text {atom }} / \phi_{\text {light }}=(c / v)^{2}
$$

if identical gratings are used for light and atom interferometers.

The presence of velocity $v$ in the Eq. (52) has two important consequences. For a space-domain interfer- ometer, the acceleration phase depends on $\tau^{2}$ while the rotation phase depends on $\tau$. Therefore slow atoms are particularly advantageous for sensing acceleration, but fast atoms (beams) offer competitive sensitivity for gyroscopes. That is why the best gravimeters use cold atoms, and the best gyroscopes use thermal atomic beams. From the vector notation in Eq. (52) one can see that reversing the atom velocity switches the sign for the rotation phase but not the acceleration phase. This provides a method to distinguish $\Omega_{x}$ from $g_{y}$ or $g_{z}$. Kassevich used counterpropagating atom beams for this reason (Gustavson et al., 2000).

Instrument resolution is given by the response factor times the precision with which the phase shift can be measured. Since the noise-limited phase precision increases with the square root of time [as discussed in Sec. III, Eq. (19)], it is customary to report the resolution per root hertz. Instrument bandwidth is limited in part by the desired resolution and also simply by the atom's time of flight. Dynamic range can be limited by dispersion. For example, if there is a velocity spread in a spacedomain interferometer, then the resulting spread in inertial phase decreases the contrast, as discussed in Secs. III and IV. For a Gaussian distribution in phase with an rms $\sigma_{\phi}$, the contrast is reduced by the factor $C / C_{0}$ $=\left\langle e^{i \phi}\right\rangle=e^{-(1 / 2) \sigma_{\phi}^{2}}$.

Measurements of gravitational acceleration in the engineering literature are often reported in units of $\mu \mathrm{Gal}$ $\left(1 \mu \mathrm{Gal}=10^{-8} \mathrm{~m} / \mathrm{s}^{2}\right)$ or the more common unit of $g$ $\left(g \approx 9.8 \mathrm{~m} / \mathrm{s}^{2}\right)$. Many applications in geophysics are currently served with sensors that have $5 \times 10^{-9} \mathrm{~g}(5 \mu \mathrm{Gal})$ precision after averaging for $15 \mathrm{~min}$ (Allis et al., 2000). The light pulse (Raman) interferometer by Peters et al. (2001) (described in Sec. III) attains this precision in less than $1 \mathrm{~min}$. Measurements with this apparatus that show time variations in local $g$ due (mostly) to tides are shown in Fig. 53. Some variations in $g$ due to sources of geophysical interest are shown in Table III.

Gravity gradients in the engineering literature are often measured in units of $E\left(1 E=10^{-9} \mathrm{~s}^{-2} \approx 10^{-10} \mathrm{~g} / \mathrm{m}\right)$ or simply $g / \mathrm{m}$. By measuring the differential gravitational acceleration in two atom interferometers located $1 \mathrm{~m}$ apart from each other, Snadden et al. (1998) measured the Earth's gravity gradient $\left(\nabla g=3 \times 10^{-6} \mathrm{~g} / \mathrm{m}\right)$ with an uncertainty of $5 \%$ and Fixler et al. (2007) measured the change in gravity gradient caused by a $540-\mathrm{kg}$ source mass of $\mathrm{Pb}\left(\nabla g=8 \times 10^{-9} \mathrm{~g} / \mathrm{m}\right)$ with an uncertainty of $0.3 \%$. Related measurements are also described by Foster et al. (2002); Kasevich (2002); McGuirk et al. (2002). Second-order phase shifts due to the Earth's gravity, gravity-gradients, and centrifugal and Coriolis forces due to the Earth's rotation have been identified by Bertoldi et al. (2006); Bongs et al. (2006); Dubetsky and Kasevich (2006) (see Table IV).

Historical background. The first measurements of $g$ with a matter-wave interferometer were done with neutrons by Colella et al. (1975). An early proposal for atom interferometer measurements of $g$ by Clauser (1988) was followed by several demonstrations with rapidly improv- 


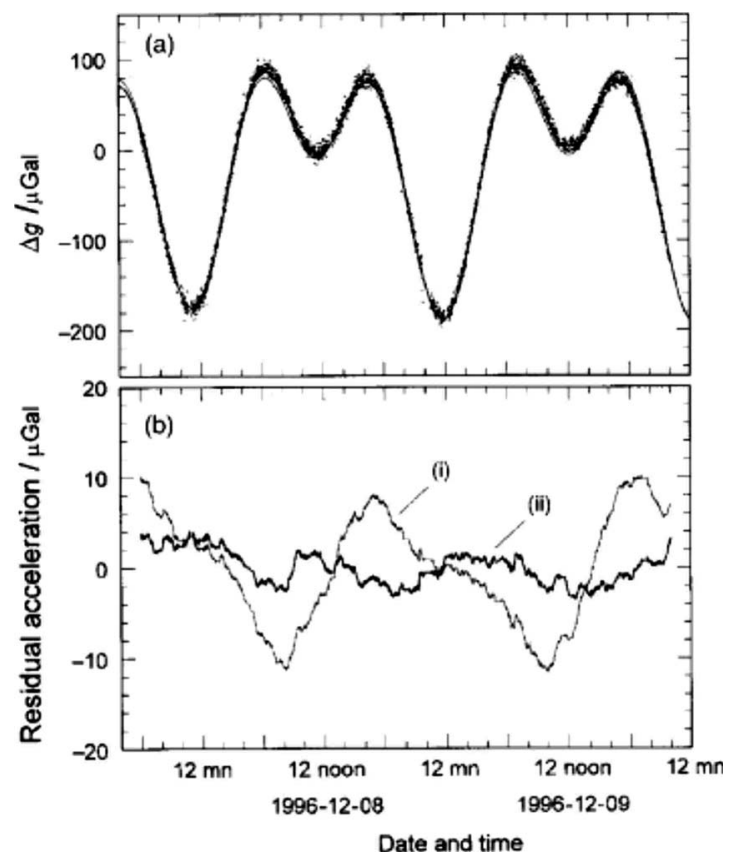

FIG. 53. Measurements of $g$. (a) Two days of gravity data. Each data point represents a 1-min gravity measurement. The solid lines represent two different tidal models. (b) The residuals of the data with respect to a tidal model where (i) the Earth is modeled as a solid elastic object and (ii) the effects of ocean loading of the Earth are taken into account. From Peters et al., 2001.

ing resolution and accuracy (Kasevich and Chu, 1992; Peters et al., 1997, 1999, 2001; Schmiedmayer et al., 1997; Young et al., 1997). An atom beam sensor for little $g$ based on the classical moire effect was also constructed with three material gratings by Oberthaler, Bernet, et al. (1996).

In 1913, Sagnac (1913a, 1913b) made his famous lightinterferometric measurement of platform rotation. Michelson (1925) measured the rotation rate $\Omega_{e}$ of the Earth, with a large optical interferometer. The Sagnac effect with neutron and electron interferometers has also been demonstrated (Werner et al., 1979; Hasselbach and Nicklaus, 1993). Atom interferometer gyroscopes were proposed early on by Clauser (1988). An atom interferometer Sagnac gyroscope was first built by Riehle et al. (1991) and large improvements in sensitivity were

TABLE III. List of geophysical sources of change in $g$ (Allis $e t$ al., 2000; Peters et al., 2001; Sasagawa et al., 2003).

\begin{tabular}{ll}
\hline \hline Gravitation source & Magnitude \\
\hline Tides at Stanford, CA & $2 \times 10^{-7} \mathrm{~g}$ \\
$1000 \mathrm{~kg}, 1.5 \mathrm{~m}$ away & $3 \times 10^{-9} \mathrm{~g}$ \\
Loaded truck $30 \mathrm{~m}$ away & $2 \times 10^{-9} \mathrm{~g}$ \\
Elevation variation of $1 \mathrm{~cm}$ & $3 \times 10^{-9} \mathrm{~g}$ \\
Ground water fluctuation of $1 \mathrm{~m}$ & $5 \times 10^{-9} \mathrm{~g}$ \\
$10^{8} \mathrm{~kg}$ of oil displacing salt at $1 \mathrm{~km}$ & $5 \times 10^{-7} \mathrm{~g}$ \\
\hline \hline
\end{tabular}

TABLE IV. Rotation rates due to various causes.

\begin{tabular}{ll}
\hline \hline Cause & \multicolumn{1}{c}{ Rotation rate $(\mathrm{rad} / \mathrm{s})$} \\
\hline Earth's rotation & $\Omega_{e}=7.2 \times 10^{-5}$ \\
Tidal drag in 1 yr & $\delta \Omega_{e}=10^{-13}$ \\
Lense-Thirring & $\Omega_{L T}=10^{-14}$ \\
Geodetic effect & $\Omega_{G D}=10^{-12}$ \\
\hline \hline
\end{tabular}

demonstrated by Lenef et al. (1997) and Gustavson et al. (1997, 2000).

\section{B. Newton's constant $G$}

Newton's constant $G$ is the least accurately known fundamental constant. The 2005 CODATA value of $G$ has a precision of $1.4 \times 10^{-4}$ (Mohr and Taylor, 2005), although several individual experiments have recently claimed precision better than this (Gundlach and Merkowitz, 2000; Quinn et al., 2001). Atom interferometry is a relatively new method to measure $G$, and may soon provide comparable precision to the CODATA value.

The Kasevich group determined $G$ with a precision of $3 \times 10^{-3}$ (Fixler et al., 2007), and the Tino group reported a value for $G$ with a precision of $1 \times 10^{-2}$ (Bertoldi et al., 2006). Both of these groups use two atom interferometer gravimeters and a movable source mass of order $500 \mathrm{~kg}$. The Tino group plans to extend their precision to the $10^{-4}$ level. Methods to measure $G$ with atom interferometry have also discussed by Kasevich (2002); McGuirk et al. (2002); Fattori et al. (2003); Stuhler (2003).

\section{Tests of relativity}

In accord with Einstein's principle of equivalence, atomic mass $m$ does not enter into Eq. (52). However, theories that go beyond Einstein's general relativity motivate the search for composition-dependent gravitational forces. The principle of equivalence has been tested accurately enough to state $\Delta g / g=1.2 \pm 1.7 \times 10^{-7}$ for the two different $\mathrm{Rb}$ isotopes (Fray et al., 2004) and there are plans based on current technology to increase the precision of this test to $\Delta g / g \sim 10^{-15}$ (300 times better than current limits from any method) (Dimopoulos et $a l ., 2007)$. There are also proposals to measure $g$ for antimatter using interferometry of positronium or antihydrogen.

Searches for a breakdown of the $1 / r^{2}$ law are another test of general relativity, in this case motivated by string theories and the possibility of compact dimensions. Experiments to detect non-Newtonian gravitational potentials with multiple atom interferometers located at different distances from the Earth's center have been discussed by Mathevet et al. (2002) and Dimopoulos et al. (2007). Experiments to search for a breakdown of the $1 / r^{2}$ law at micrometer length scales using atom interfer- 
ometry were discussed by Dimopoulos and Geraci (2003) and Ferrari et al. (2006).

The gravitational scalar AB effect would be an interesting test at the intersection of quantum mechanics and gravity. If a 5-cm-radius lead sphere has a small hole in the center, Cs atoms placed there have a frequency shift of about $7 \mathrm{~Hz}$. Thus atoms at the top of their trajectory could easily experience a phase shift $\sim 10 \mathrm{rad}$, enabling a quantum measurement of the gravitational potential. If the lead is assembled around the atoms in one interferometer arm, or if the lead is moved into position quickly compared to the atoms' transit time, the effects of the gravitational field (force) can largely be eliminated, making this a sensitive measure of gravitational potential.

Atom interferometer rotation sensors in low Earth orbit should be able to measure the geodetic effect and possibly the Lense-Thirring rotation. According to special relativity, freely falling gyroscopes orbiting in the vicinity of the Earth will experience the geodetic effect caused by the motion of the gyroscope in the gravitational field of the Earth (Schiff, 1960; Jentsch et al., 2004) For low Earth orbit, the rotation rate induced by the geodetic effect is $10^{-12} \mathrm{rad} / \mathrm{s}$, and is independent of the Earth's rotation rate.

The Lense-Thirring rotation [Thirring (1918)] is a general relativity effect that causes a gyroscope to rotate relative to the fixed stars due to a massive rotating body being nearby. It is also called the gravitomagnetic effect. In low Earth orbit (700-km altitude), this can be as large as $10^{-14} \mathrm{rad} / \mathrm{s}$ and depends on the orientation of the Earth's spin. Measurements of both the geodetic effect and the Lense-Thirring effect is the objective of future space borne atom interferometer missions (Jentsch et al., 2004).

While optimistic proposals to detect gravity waves using atom interferometers (Chiao and Speliotopoulos, 2004; Tino and Vetrano, 2007) have been questioned (Roura et al., 2006) it is clear that precision measurements of $G, g$, and tests of the $1 / r^{2}$ law are possible using various atoms as test particles.

\section{Interferometers in orbit}

In addition to ultraprecise atomic clocks to improve the atomic clocks already aloft for the GPS system, physics experiments that could benefit from being in space include measurements of the gravitational redshift, tests of Einstein's equivalence principle, mapping the Lense-Thirring effect close by the Earth, and measurements of $h / m$.

NASA works on these goals with the laser cooled atom physics (LCAP) and ultraprecise primary atomic reference clocks in space (PARCS) programs planned for the international space station (Lee and Israelsson, 2003). The European Space Agency's HYPER-precision atom interferometry in space project is described in several articles in General Relativity and Gravitation, Vol. 36, No. 10, (2004) starting with Jentsch et al. (2004); see Fig. 54.

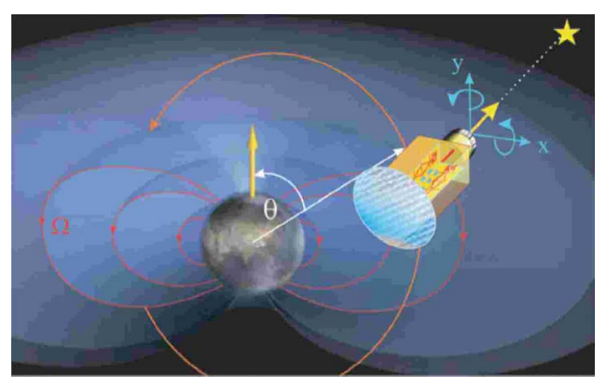

FIG. 54. (Color online) The mission scenario: HYPER, which follows a nearly polar circular orbit, will measure with two atomic gyroscopes the two characteristic components of the Lense-Thirring rotation as a function of latitudinal position $\theta$. From Jentsch et al., 2004.

\section{E. Fine structure constant and $\hbar / M$}

One of the highest precision atom interferometry experiments is the measurement of $\hbar / m_{\text {atom }}$. This leads to a measurement of the molar Planck constant $N_{A} h$ $=\left(M_{\text {atom }} / m_{\text {atom }}\right) h \times 1000$, where $M_{\text {atom }}$ is the atomic weight of the atom in grams and the factor of 1000 comes in converting the atomic mass into kilograms. This was done by Weiss et al. $(1993,1994)$ and Wicht et al. (2002) and more recently by Biraben and co-workers (Battesti et al., 2004; Clade et al., 2006). Both groups achieved a precision of $\sim 14$ parts per billion (ppb) by measuring the velocity change of an atom due to the photon recoil (from emission or absorption). As we discuss, these measurements lead to a value for the fine structure constant at $\sim 7 \mathrm{ppb}$ when combined with other measurements.

The underlying physics, first exploited using neutrons by Kruger et al. (1995), is based on the de Broglie wavelength

$\lambda_{\mathrm{dB}}=h / m v$.

A simultaneous measurement of both $\lambda_{\mathrm{dB}}$ and $v$ gives $h / m$, where $m$ is the mass of the neutron of the particular atom used in the experiment. In the interferometer experiment of $\mathrm{Chu}$, the measured quantity is essentially the frequency with which an atom with the recoil velocity (from absorbing a photon of wave vector $k$ )

$$
v_{\text {rec }}=\hbar k / m_{\mathrm{Cs}}
$$

crosses the fringes in a standing wave,

$$
\omega=2 \pi v_{\mathrm{rec}} / \lambda_{\mathrm{dB}} \sim \hbar k^{2} / m_{\mathrm{Cs}},
$$

where we have replaced $\lambda_{\mathrm{dB}}$ with the wavelength of the light causing the recoil. In the Biraben experiment, the Doppler shift associated with this recoil is measured:

$$
\omega_{D}=k v_{\text {rec }}=\hbar k^{2} / m_{\mathrm{Rb}} \text {. }
$$

These frequencies are equal to the recoil frequency (typically $10 \mathrm{kHz}$ ) derived earlier from consideration of the energy of recoil.

In the actual experiments, the measured frequency is several times the recoil frequency. Measuring the small 
recoil frequency to ppb accuracy is impossible given a maximum free fall time for the atoms of a fraction of a second. Hence both experiments increase the measured velocity by contriving to add recoil velocities from the absorption of many photons. In the $\mathrm{Chu}$ experiments these are added in using up to 60 Raman pulses or adiabatic (stimulated adiabatic rapid passage) transfers, in the Biraben experiment by accelerating an optical lattice into which the atoms are embedded. Although the initial and final lattice speeds are not quantized, the atoms accelerated in them always absorb an integral number of lattice momenta (sum of momenta in the two laser beams forming the moving lattice) —up to 900 photon momenta in Clade et al. (2006).

Both of these experiments are essentially measurements of velocity, using the combined techniques of atom optics (to add velocity) and atom interferometry to detect it. This is indicated by the fact that the signal increases linearly with the extra velocity. An interferometer configuration that uses contrast interferometry to measure the recoil energy has been proposed and demonstrated (Gupta et al., 2002). It shows the quadratic dependence of phase shift on photon number (velocity) expected for an energy measurement, and therefore requires that less additional momentum be added to achieve the same precision.

An important consequence of the $\hbar / m$ measurement is to provide a high-accuracy route to the determination of the fine structure constant $\alpha$. This is based on the relationship

$$
\alpha^{2}=\left(e^{2} / \hbar c\right)^{2}=\left(2 R_{\infty} / c\right) h / m_{e} .
$$

Combining atom interferometer results with independent measurements of the optical frequency $(\omega=c k)$ (Gerginov et al., 2006), the mass ratios $m_{\mathrm{Cs}} / m_{p}$ (Riehle et al., 1996; Bradley et al., 1999) and $m_{p} / m_{e}$ (Mohr and Taylor, 2005) and the Rydberg $R_{\infty}$ (Mohr and Taylor, 2005) gives a value of the fine structure constant.

The determination of $\alpha$ from $h / m_{\mathrm{Cs}}$ has a precision of $7 \mathrm{ppb}$ (Wicht et al., 2002), and from Rb of $6.7 \mathrm{ppb}$ (Clade et al., 2006). Thus this route already offers the second most accurate value of $\alpha$ (after the measurement of $g$ -2 for the electron), and therefore allows the most precise comparison across different subfields of physics, as shown in Fig. 55. It essentially offers a comparison of QED with such things as the de Broglie wavelength relationship and calculations of atomic structure in hydrogen. Such cross-field comparisons are important for the unity and global understanding of physics, and provide one of the few routes to discover underlying errors in an isolated subfield. It is interesting to note that several of the values appearing in Fig. 55 have been substantially re-evaluated between 1998 and 2002, which proves that the fine structure constant is not so well known (it is known mostly due to the electron spin anomaly).

No precision experiment is easy, and the $h / m$ measurements discussed here experience difficulties from vibration that changes the velocity of the reference light waves [the scheme by Gupta et al. (2002) demonstrated

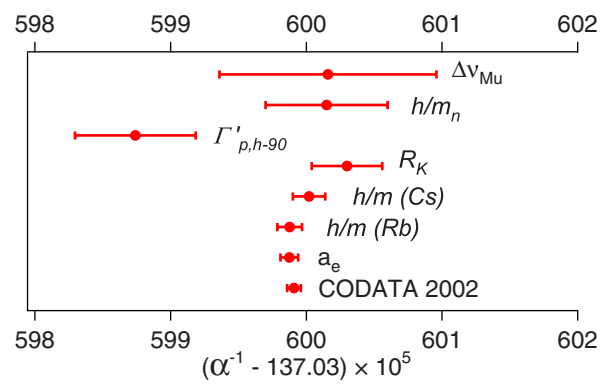

FIG. 55. (Color online) Determinations of the fine structure constant $\alpha$ by several methods. The value from $h / m(\mathrm{Cs})$ is from atom interferometry (Wicht et al., 2002) and the $h / m(\mathrm{Rb})$ value is determined with Bloch oscillations (Clade et al., 2006). References are given in Mohr and Taylor (2005) for the values obtained from measuring the muonium hyperfine splitting $\left(\Delta \nu_{\mathrm{Mu}}\right)$, measuring the von Klitzing constant with the quantum Hall effect $\left(R_{K}\right)$, measuring recoil velocity of neutrons Bragg reflecting from silicon crystals $\left(h / m_{n}\right)$, or measuring gyromagnetic ratios $\left(\Gamma_{p, h-90}^{\prime}\right)$, or measuring electron and positron anomalies $\left(a_{e} \equiv g_{e} / 2-1\right)$. From Mohr and Taylor, 2005 .

vibrational insensitivity], stray field gradients, etc. Other sources of noise and systematic error in these experiments include the index of refraction for light due to the atomic ensemble (Wicht et al., 2002; Campbell et al., 2005), ac Stark shifts for the atomic energy levels due to the laser fields (Wicht et al., 2002, 2005), beam misalignment and wave-front curvature (Wicht et al., 2002; Gibble, 2006), and mean field shifts for the atomic energy states due to interaction with nearby atoms (Gupta et al., 2002; Le Coq et al., 2006).

The accuracy of these atom interferometric methods for measuring $h / m$ is still increasing due to the rapid overall progress in atom interferometry with cold atoms and because sources of error are being understood and overcome. It is certain that the accuracy of $h / \mathrm{m}$ will soon be improved in both $\mathrm{Rb}$ and $\mathrm{Cs}$, which employ significantly different atom optics methods. This might mean that the real limit of confidence in this route to $\alpha$ would be in the measurements of the atomic masses of $\mathrm{Rb}$ and $\mathrm{Cs}$ for which there is only one high-precision measurement (Bradley et al., 1999), and that had unexplained systematic errors at the 0.2-ppb level. We know of no other experiments planned that could check these heavy masses, whereas there are two or more measurements of both the Rydberg and electron mass ratios that are consistent.

Here we provide more detail on the Chu group experiment, described by Weiss et al. $(1993,1994)$ and Wicht et al. (2002). To determine $h / m$ they measured the relative frequency of the final $\pi / 2$ pulses in two different atom interferometers (Fig. 56). The frequency difference between the resonances of the two interferometers depends only on conservation of energy and conservation of momentum. As an example of the recoil shift, consider a simplified experiment (Weiss et al., 1994) where an atom (with mass $m$ ) in state $|a\rangle$ with zero velocity in the laboratory frame first absorbs a photon from a leftward propagating laser beam with frequency $\omega$. The 

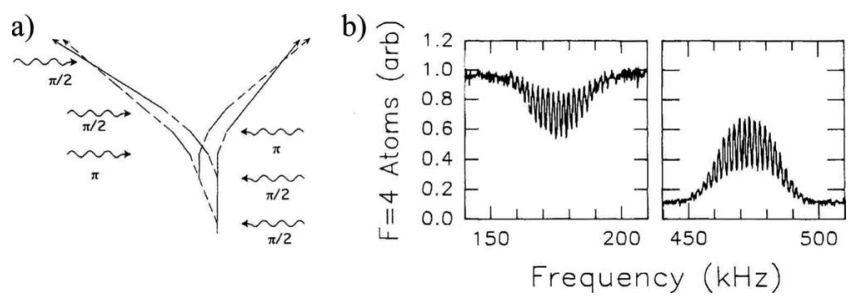

FIG. 56. Measurement of recoil frequency. (a) A double interferometer where the two interfering pairs have their velocities shifted with respect to each other by four photon recoils. Solid lines indicate atoms in internal state $a$, and dashed lines represent internal state $b$. (b) Sets of Ramsey fringes displaced by $2 \pi \Delta \omega$ (due to $8 \pi$ pulses in the middle of the interferometers). Only the frequency of the final two $\pi / 2$ pulses is scanned. From Weiss et al., 1994.

atom recoils by $\hbar k / m$ and the process has a resonance condition

$$
\omega-\omega_{a b}=\hbar k^{2} / 2 m,
$$

where $\hbar \omega_{a b}$ is the energy difference between atomic states $|b\rangle$ and $|a\rangle$ at rest. The atom can then be deexcited by a rightward propagating beam with frequency $\omega^{\prime}$. It receives another velocity kick $\hbar k^{\prime} / m$ in the same direction and the new resonance condition is

$$
\omega^{\prime}-\omega_{a b}=-\hbar k k^{\prime} / m-\hbar k^{\prime 2} / 2 m .
$$

The two resonances are shifted relative to each other by

$$
\Delta \omega=\omega-\omega^{\prime}=\hbar\left(k+k^{\prime}\right)^{2} / 2 m .
$$

Furthermore, the resonance condition for an atom in $|b\rangle$ moving with velocity $(N-1) v_{\text {rec }}$ towards a laser beam is

$$
\omega^{\prime}-\omega_{a b} \approx\left(\hbar k^{2} / 2 m\right)\left[(N-1)^{2}-(N)^{2}\right]
$$

so that

$$
\Delta \omega \approx N \hbar k^{2} / m,
$$

where $N$ is the total number of photon recoil momenta imparted to the atom and the approximation comes from the fact that $k^{\prime} \approx k$. This shows why $\Delta \omega$ depends linearly on $N$.

\section{ATOMIC PHYSICS APPLICATIONS}

A major motivation for atom interference experiments is to learn more about atoms and molecules themselves.

Atoms in a separated beam interferometer experience a phase shift if a uniform but different potential is applied to each arm. Thus interferometers offer sensitivity to the potentials (not just forces). This sensitivity has been used to measure the index of refraction due to other atoms and energy shifts due to electric and magnetic fields. We emphasize that de Broglie wave phase shift measurements bring spectroscopic precision to experiments where usually classical methods like beam deflection or velocity measurement were applied, as discussed in Secs. I-IV.

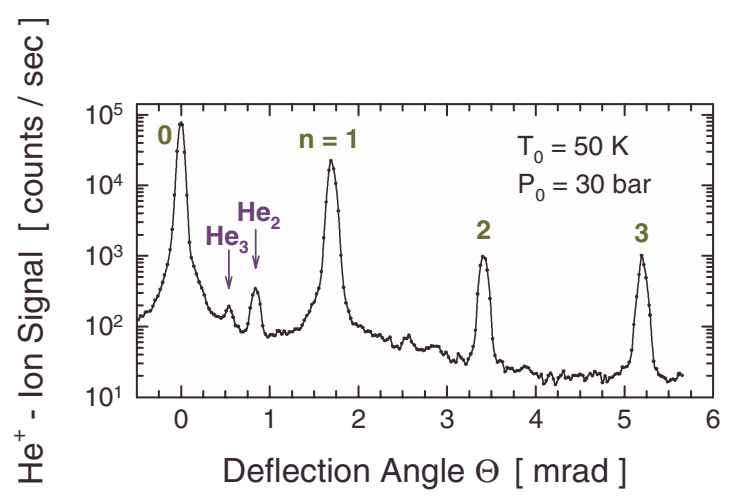

FIG. 57. (Color online) Diffraction of helium atoms and helium molecules through a nanofabricated grating. From Bruch et al., 2002.

In another application, the nanogratings used as a de Broglie wave gratings can function as a gentle spectrometer that diffracts different molecular species in a molecular beam to different angles.

The nanostructures themselves also produce potentials due to atom-surface interactions that have been measured with interferrometric techniques. For gratings with 50 -nm-wide slots, each transmitted atom must pass within $25 \mathrm{~nm}$ of a grating bar; hence the measured intensities are affected by the nonretarded vdW potential. With larger gratings, on the other hand, the CasimirPolder potential has been probed.

\section{A. Discovery of $\mathrm{He}_{2}$ molecules}

One application of coherent atom optics laid to rest a long standing argument concerning whether a stable bound state of the ${ }^{4} \mathrm{He}_{2}$ dimer exists. [The attribution of $\mathrm{He}_{2}{ }^{+}$to $\mathrm{He}_{2}$ dimers formed in cryogenic expansion by (Luo et al., 1993) primarily reported an old debate.] For this experiment a diffraction grating was used to separate and resolve ${ }^{4} \mathrm{He}_{2}$ dimers from a helium beam (Fig. 57) (Schöllkopf and Toennies, 1996). Subsequently, a grating was used as a nanosieve to measure the size of the ${ }^{4} \mathrm{He}_{2}$ dimers. The value $\langle r\rangle=6.2 \pm 1.0 \mathrm{~nm}$ was reported by Luo et al. (1996). Grisenti, Schöllkopf, Toennies, Hegerfeldt, et al. (2000) reported a value of $5.2 \pm 0.4 \mathrm{~nm}$. This size corresponds to a binding energy of $E / k_{B}=1 \mathrm{mK}$. Diffraction has also been used to study the formation of more massive clusters (Bruehl et al., 2004), and searches using this technique are underway for an Efimov-type excited state in ${ }^{4} \mathrm{He}_{3}$. This would be manifest as a particularly large $(\langle r\rangle=8.0 \mathrm{~nm})$ excited-state helium trimer (Bruehl et al., 2005; Hegerfeldt and Stoll, 2005; Stoll and Kohler, 2005).

\section{B. Polarizability measurements}

\section{Ground-state de scalar polarizability}

By inserting a metal foil between the two separated arms, as shown in Fig. 58, an electric field can be applied to a single path. The resulting de Broglie wave phase 

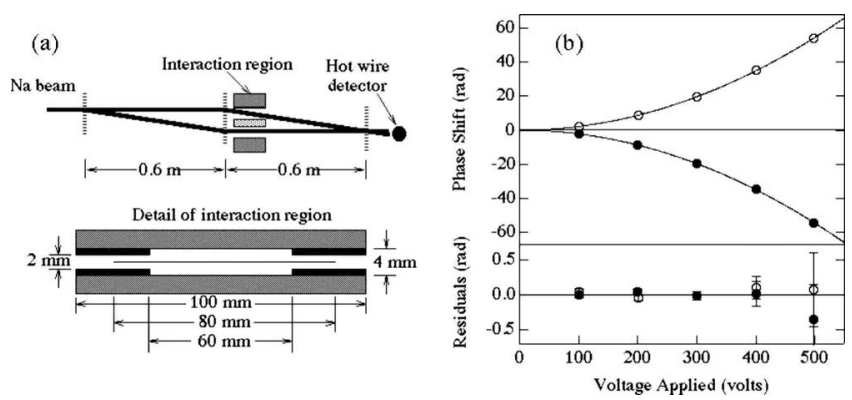

FIG. 58. Measurement of atomic polarizability. (a) Schematic of the interaction region installed behind the second grating. (b) Measured phase shifts vs applied voltage. The two different signs of the phase shift stem from the voltage being applied on either the left (open circles) or the right (filled circles) side of the interaction region (arm of the interferometer). The fit is to a quadratic and the residuals are shown on the lower graph. From Schmiedmayer et al., 1997.

shift was used to measure the static ground-state atomic polarizability of sodium $\alpha_{\mathrm{Na}}$, with a precision of $0.35 \%$ (Ekstrom et al., 1995). Similar precision has been demonstrated for $\alpha_{\mathrm{He}}$ (Toennies group) and $\alpha_{\mathrm{Li}}$ (Vigué group) using this method (Toennies, 2001; Miffre et al., 2006b, 2006c).

In this experiment a uniform electric field $\mathcal{E}$ is applied to one of the separated atomic beams, shifting its energy by the Stark potential $U=-\alpha \mathcal{E}^{2} / 2$. The static scalar ground-state polarizability $\alpha_{\text {pol }}$ is determined from the phase shift $\Delta \phi$ of the interference pattern by

$$
\alpha_{\mathrm{pol}}=\left(\Delta \phi / V^{2}\right)\left(D^{2} / L_{\mathrm{eff}}\right) 2 \hbar v,
$$

where $V$ is the voltage applied to one electrode in the interaction region, $D$ is the distance between the electrode and the septum, $v$ is the mean velocity of the atomic beam, and $L_{\text {eff }}$ is the effective interaction region length defined as

$$
(V / D)^{2} L_{\text {eff }} \equiv \int \mathcal{E}^{2} d z
$$

For an accurate determination of electric polarizability, the three factors in Eq. (68) must each be determined precisely. They are (i) the phase shift as a function of applied voltage, (ii) the geometry and fringing fields of the interaction region, and (iii) the velocity of the atoms. Ekstrom et al. (1995) had an uncertainty in each term less than $0.2 \%$.

Taking all sources of error into account, and adding statistical and systematic errors in quadrature, the static polarizability of the ground state of sodium was measured to be $\alpha_{\mathrm{pol}}=24.11 \times 10^{-24} \mathrm{~cm}^{3}$, with a fractional uncertainty of $0.35 \%$. This measurement was a nearly 30 fold improvement on the best previous direct measurement of the polarizability of sodium (Hall and Zorn, 1974) based on beam deflection.

A similar experiment for $\mathrm{He}$ was done with a threegrating Mach-Zehnder interferometer (with nanogratings) by the Toennies group. The phase stability of this interferometer was so good that the fringes could be

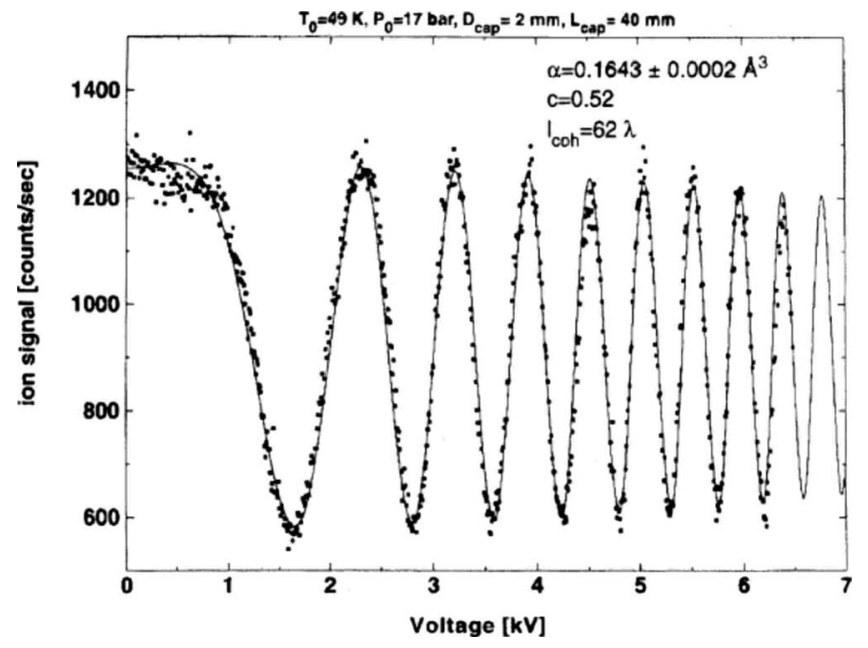

FIG. 59. Measurement of the electric polarizability of He. The gratings are held stationary while the electric field is increased. The measurement uncertainty is statistical only. Figure courtesy of J. P. Toennies and R. Bruehl.

observed directly as a function of applied electric field, while the gratings were not moved (Fig. 59). The statistical precision in $\alpha_{\mathrm{He}}$ was $0.1 \%$ (Toennies, 2001).

Using three Bragg diffraction gratings for $\mathrm{Li}$ atoms and a septum electrode, the group of Vigué measured $\alpha_{\mathrm{Li}}$ with a precision of $0.66 \%$ (Miffre et al., 2006b, 2006c). Using a Talbot-Lau interferometer with a field gradient, Berninger et al. (2007) measured the polarizability of fullerenes $\alpha_{\mathrm{C}_{60}}$ and $\alpha_{\mathrm{C}_{70}}$ with a statistical precision of $1 \%$ and an overall uncertainty of $6 \%$.

Because atom interferometry gives sub-hertz precision on the energy shift of the atomic ground state, ratios of polarizabilities for different species can be accurately determined with multispecies atom interferometers. Uncertainty in the interaction region geometry would then be less significant because the quantity $D^{2} / L_{\text {eff }}$ in Eq. (68) cancels out in a ratio of, for example, $\alpha_{\mathrm{Rb}} / \alpha_{\mathrm{Li}}$. The ratio of velocities of the two species would still need to be measured, or taken into account. Thus improved precision in measurements of $\alpha_{\text {pol }}$ may come from using an engineered phase shift to cancel the velocity dependence of the polarizability phase shift. This is known as dispersion compensation (Roberts et al., 2004). Velocity multiplexing (Hammond et al., 1995) and Schmiedmayer and magnetic re-phasing (Schmiedmayer et al., 1994) are other approaches for dealing with the experimental spread in velocity. With these improvements it seems feasible to perform polarizability measurements with uncertainties in the $10^{-4}$ range.

This precision offers an excellent test of atomic theory, because theoretical uncertainties in light elements like $\mathrm{Li}$ are orders of magnitude smaller than in heavier alkali metals. Polarizability $\alpha_{\text {pol }}$ of an atomic state can be expressed as a sum over dipole matrix elements: 

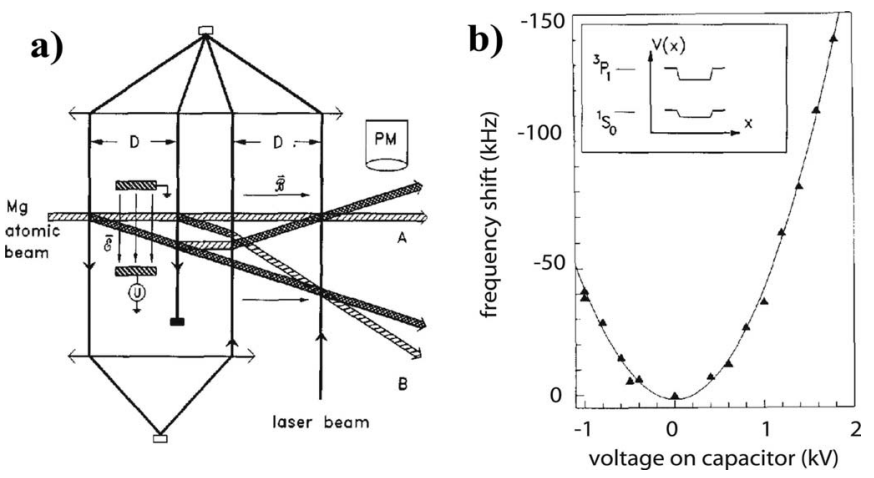

FIG. 60. Optical Ramsey-Bordé interferometer for measuring polarizability differences. (a) Schematic of the atom interferometer with a capacitor. (b) Frequency shift of the interference pattern vs voltage across the capacitor. The fit is a parabola. The inset shows the energy levels as a function of position through the capacitor. From Rieger et al., 1993.

$$
\alpha_{\mathrm{pol}}=e^{2} \sum_{j \neq i} \frac{\langle i|\mathbf{r}| j\rangle\langle j|\mathbf{r}| i\rangle}{E_{j}-E_{i}}
$$

where $E_{j}$ is the energy of state $|j\rangle$. Accurate calculation of static dipole polarizabilities for heavy atoms still remains a great challenge because electron correlation and relativistic effects become increasingly important for heavy atoms. Major theoretical efforts so far have included the relativistic Hartree-Fock approach, manybody perturbation theory, density functional theory, and relativistic coupled-cluster technique. Several calculations of atomic polarizability all show the need for precise experimental measurements (Bonin and Kadarkallen, 1994; Kharchenko et al., 1997; Rerat et al., 1998; Derevianko et al., 1999; Lim et al., 1999; Safronova et al., 1999; Hohm, 2000; Kronic et al., 2001; Maroulis, 2001; Derevianko and Porsev, 2002; Thakkar and Lupinetti, 2005).

\section{Transition dc and ac Stark shifts}

When two paths have different internal states, e.g., in an optical Ramsey-Bordé interferometer, then a uniform electric field applied to both paths makes phase shifts proportional to the difference of polarizability of the two states. (This is similar to what can be measured with laser spectroscopy.) For example, the dc Stark shift of the magnesium $3 s^{2}\left(1 S_{o}\right)-3 s 3 p\left({ }^{3} p_{1}\right)$ intercombination line was measured by subjecting both arms of an atom interferometer to a constant electric field. The Stark energy perturbation provides two different potentials in the two arms of the interferometer. The resulting relative phase shift (Fig. 60) corresponds to a difference of $-(8 \pm 1)$ $\mathrm{kHz}(\mathrm{kV} / \mathrm{cm})^{-2}$ in the polarizabilities of the ${ }^{1} S_{o}$ and ${ }^{3} P_{1}$ $(m=1)$ states. (Rieger et al., 1993).

A related approach was used to measure the difference between the polarizabilities of the ${ }^{3} P_{1}$ state and the ${ }^{1} S_{o}$ state of Ca to be $\alpha\left({ }^{3} P_{1}\right)-\alpha\left({ }^{1} S_{o}\right)=(13 \pm 2) \times 10^{24} \mathrm{~cm}^{3}$ (Morinaga, Nakamura, et al., 1996).
The ac Stark shift of the $4 s_{2}{ }^{1} S_{o}-4 s 4 p^{3} P_{1}$ line in ${ }^{40} \mathrm{Ca}$ was measured with a time-domain Ramsey-Bordé atom interferometer (in a magneto-optical trap) for perturbing laser wavelengths between 780 and $1064 \mathrm{~nm}$ (Degenhardt et al., 2004). ac Stark shifts have also been observed in a double-well interferometer (Shin et al., 2004).

\section{Index of refraction due to dilute gases}

A physical membrane separating the two paths allows insertion of a gas into one path of the interfering wave, enabling a measurement of the index of refraction for atom waves traveling through a dilute gas caused by the collision-induced phase shift. Measurements have been presented by Schmiedmayer et al. $(1995,1997)$; and these experiments have been discussed by Audouard et al. (1995, 1997); Vigué (1995); Forrey et al. (1996, 1997); Champenois et al. (1997); Kharchenko and Dalgarno (2001); Blanchard et al. (2003)

Scattering makes a wave function evolve as

$$
\psi \stackrel{r \rightarrow \infty}{\longrightarrow} e^{i \mathbf{k} r}+f\left(\mathbf{k}, \mathbf{k}^{\prime}\right) e^{i \mathbf{k}^{\prime} r} / r,
$$

where the scattering amplitude $f$ contains all information about the scattering process (Sakurai, 1994). The complex index of refraction $n$ due to a gas of scattering centers is related to $f$ by summing the scattered amplitudes in the forward direction (Newton, 1966), resulting in

$$
n=1+\left(2 \pi N / \mathbf{k}^{2}\right) f(\mathbf{k}, \mathbf{k}),
$$

where $N$ is the gas density. Atoms propagating through the gas are phase shifted and attenuated by the index

$$
\psi(z)=\psi(0) e^{i n k z}=\psi(0) e^{i k z} e^{i \Delta \phi(N, z)} e^{-(N / 2) \sigma_{\mathrm{tot}} z} .
$$

The phase shift due to the gas,

$$
\Delta \phi(N, z)=\left(2 \pi N k z / k_{\mathrm{cm}}\right) \operatorname{Re}\left[f\left(k_{\mathrm{cm}}\right)\right],
$$

is proportional to the real part of the forward scattering amplitude, while the attenuation is related to the imaginary part. Attenuation is proportional to the total scattering cross section which is related to $\operatorname{Im}[f]$ by the optical theorem

$$
\sigma_{\mathrm{tot}}=\left(4 \pi / k_{\mathrm{cm}}\right) \operatorname{Im}\left[f\left(k_{\mathrm{cm}}\right)\right] .
$$

Measurements of phase shift as a function of gas density are shown in Fig. 61.

The ratio of the real and imaginary parts of the forward scattering amplitude is a natural quantity to measure and compare with theory. This ratio,

$$
\rho(k)=\frac{\Delta \phi(N)}{\ln [A(N) / A(0)]}=\frac{\operatorname{Re}[f(k)]}{\operatorname{Im}[f(k)]},
$$

where $A$ is the fringe amplitude, gives orthogonal information to the previously studied total scattering cross section. In addition it is independent of the absolute pressure in the scattering region and therefore much better to measure. 


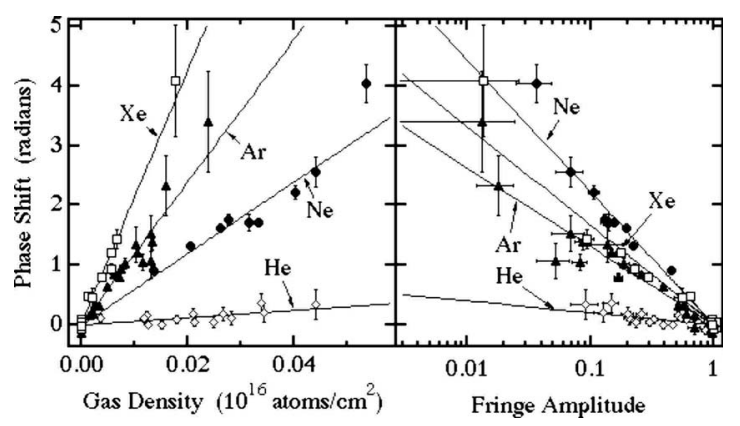

FIG. 61. Index of refraction measurements. Left: Phase shift $\Delta \phi$ as a function of gas density $N$ for different gas samples. Right: Phase shift vs fringe amplitude. The fringe amplitude is proportional to $e^{-N \sigma_{\text {tot }} z / 2}$. From Schmiedmayer et al., 1997.

The ratio $\rho(k)$ shows structure as a function of $k$ known as glory oscillations ${ }^{13}$ (Fig. 62). These were predicted by Audouard et al. $(1995,1997)$ and Forrey et al. (1997) and observed by Roberts et al. (2002). Measurements of $\rho(k)$ plotted as a function of $\mathrm{Na}$ beam velocity $v$ for target gases of $\mathrm{Ar}, \mathrm{Kr}, \mathrm{Xe}$, and $\mathrm{N}_{2}$ are shown in Fig. 62.

To compare these measurements with predictions based on various potentials $V(r)$, the forward scattering

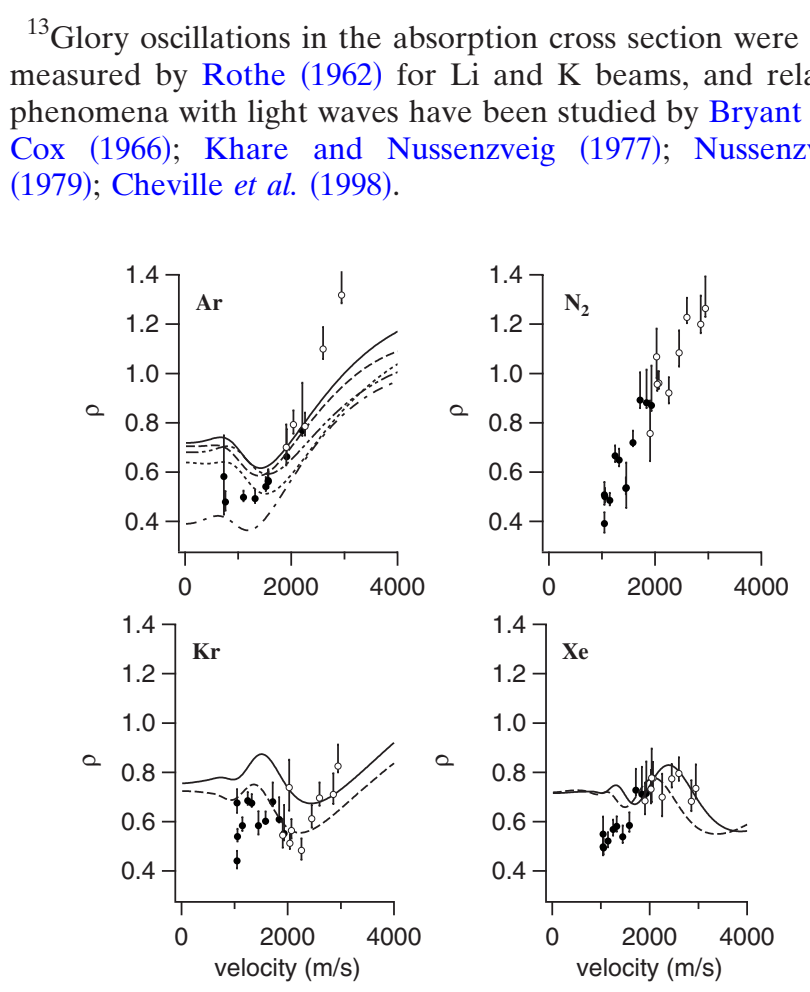

FIG. 62. $\rho$ as measured for $\mathrm{Na}$ waves in $\mathrm{Ar}, \mathrm{Kr}, \mathrm{Xe}$, and $\mathrm{N}_{2}($ using $200 \mathrm{~nm}$ gratings, $\bigcirc 100 \mathrm{~nm}$ ), showing evidence of glory oscillations in comparison to $\rho$ as derived from predicted potentials: Na-Ar (Champenois et al., 1997) (-), (Duren and Groger, 1978) (- - ), (Forrey et al., 1997) ( *), (Tellinghuisen et al., 1979) $(\cdot-\cdot-)$, (Tang and Toennies, 1977) (*- - *-); Na-Kr (Champenois et al., 1997) (-), (Duren et al., 1968) (-- -); and Na-Xe (Baumann et al., 1992) (-), (Duren et al., 1968) (- - -). From Roberts et al., 2002). amplitude was computed using the standard partial wave treatment and the WKB approximation. Predictions for $\rho$ must also include an average over the distribution of velocities in the gas sample, and this damps the glory oscillations as discussed by Champenois et al. (1997) and Forrey et al. (1997). Figure 62 shows calculations of $\rho(v)$ based on predictions of $V(r)$ for $\mathrm{Na}-\mathrm{Ar}, \mathrm{Na}-\mathrm{Kr}$, and $\mathrm{Na}-\mathrm{Xe}$ derived from spectroscopic measurements and beam scattering experiments.

The motivation for studying the phase shift in collisions is to add information to long-standing problems such as inversion of the scattering problem to find the interatomic potential $V(r)$, interpretation of other data that are sensitive to long-range interatomic potentials, and description of collective effects in a weakly interacting gas (Chadan and Sabatier, 1989; Stoof, 1991; Bagnato et al., 1993; Lett et al., 1993; Cline et al., 1994; Moerdijk and Verhaar, 1994; Moerdijk et al., 1994; Walker and Feng 1994). The glory measurements of $\rho$ are sensitive to the shape of the potential near the minimum, where the transition from the repulsive core to the van der Waals potential is poorly understood. Measurements of $\rho(k)$ also give information about the rate of increase of the interatomic potential $V(r)$ for large $r$ independently of the strength of $V(r)$. The real part of $f$ was inaccessible to measurement before the advent of separated beam atom interferometers. Controlled collisions as phase shifting tools are now widely discussed in the context of quantum computing.

\section{Casimir-Polder (atom-surface) potentials}

Atom-surface interactions are important in a wide range of nanoscale phenomena, including gas adsorption, atomic force microscopy, quantum reflection, atom chips, and many topics in biophysics and chemistry. Yet in many situations the forces are difficult to predict $a b$ initio. Single atoms passing within $50 \mathrm{~nm}$ of a dielectric surface represent a middle ground, where theoretical calculations are tractable, and precision measurements are becoming possible. Here we describe some landmark theoretical contributions to this field and then survey measurements done with coherent atom optics.

After J. D. van der Waals suggested modifications to the equation of state for gases to allow for atom-atom interactions (which he did in 1873), London (1937) calculated the strength of interactions between two polarizabile atoms using quantum mechanics, and similar ideas were used to describe atom-surface interactions (Lennard-Jones, 1932). Casimir and Polder (1948) generalized the theory of atom-surface interactions to include the effect of retardation, and Lifshitz (1956) modified this theory to allow for a surfaces with a dielectric permittivity. Since then, hundreds of theoretical works used quantum electrodynamics to predict the interaction potential for real atoms near real surfaces.

The Casimir-Polder potential for an ideal surface is (Casimir and Polder, 1948; Sukenik et al., 1993) 


$$
U(r)=\frac{1}{4 \pi \alpha r^{4}} \int_{0}^{\infty} \alpha_{\text {pol }}(i x / \alpha r) e^{-2 x}\left[2 x^{2}+2 x+1\right] d x,
$$

where $\alpha_{\text {pol }}$ is atomic polarizability (evaluated as a function of imaginary frequency), $r$ is the distance to the surface, and $\alpha$ is the fine structure constant. This has well-known limits of the van der Waals (vdW) regime,

$$
r \rightarrow 0, \quad U(r)=\frac{\hbar}{4 \pi r^{3}} \int_{0}^{\infty} \alpha_{\mathrm{pol}}(i \omega) d \omega \equiv \frac{C_{3}}{r^{3}},
$$

and the retarded regime

$$
r \rightarrow \infty, \quad U(r)=2 h c \alpha_{\text {pol }}(0) / 32 \pi \epsilon_{0} r^{4} \equiv-K_{4} / r^{4} .
$$

Marinescu et al. (1997) evaluated $U(r)$ for sodium atoms at arbitrary distances from a perfectly conducting half space, using a single electron (Lorenz oscillator) model of the atom. Derevianko et al. (1999) calculated $C_{3}$ for the alkali-metal atoms using the best available model of frequency-dependent atomic polarizability. It is noteworthy that $18 \%$ of the interaction potential between sodium atoms and a perfect mirror is due to excitations of the core electrons. The one-electron (Lorenz) oscillator model yields $C_{3}=\hbar \omega_{0} \alpha_{\text {pol }}(0) / 8$ with $\alpha_{\text {pol }}(0)$ $=e^{2} / \omega_{0}^{2} m_{e}\left[4 \pi \epsilon_{0}\right]$, where $\omega_{0}$ is the resonance frequency and $m_{e}$ is the electron mass. This one-electron model for sodium atoms and a perfectly conducting surface gives $C_{3}=6.3 \mathrm{meV} \mathrm{nm}^{3}$, while the calculation with many electrons gives $C_{3}=7.6 \mathrm{meV} \mathrm{nm}^{3}$. The Lifshitz formula

$$
C_{3}=\frac{\hbar}{4 \pi} \int_{0}^{\infty} \alpha_{\mathrm{pol}}(i \omega) \frac{\epsilon(i \omega)-1}{\epsilon(i \omega)+1} d \omega
$$

reduces $C_{3}$ even further. For sodium and silicon nitride the Lifshitz formula gives $C_{3}=3.2 \mathrm{meV} \mathrm{nm}^{3}$. Spruch and Tikochinsky (1993) and Zhou and Spruch (1995) elaborated on $U(r)$ for arbitrary $r$ and surfaces composed of multiple layers.

Several experiments can now test these predictions. Atoms transmitted through a cavity (Anderson et al., 1988; Sukenik et al., 1993), atoms diffracted from a material grating (Grisenti et al., 1999; Grisenti, Schöllkopf, Toennies, Manson, et al., 2000; Shimizu, 2001; Bruehl et al., 2002; Cronin and Perreault, 2004; Perreault et al., 2005), atoms undergoing quantam reflection (Anderson et al., 1986; Berkhout et al., 1989; Shimizu, 2001; Shimizu and Fujita, 2002a; Druzhinina and DeKieviet, 2003; Pasquini et al., 2006), atoms reflecting from evanescent waves near surfaces (Hajnal et al., 1989; Kaiser et al., 1996; Westbrook et al., 1998; Esteve et al., 2004), atoms trapped near surfaces (Lin et al., 2004; McGuirk et al., 2004; Harber et al., 2005), and atoms in interferometers (Brezger et al., 2002; Kohno et al., 2003; Nairz et al., 2003; Perreault and Cronin, 2005, 2006) have been used to measure atom-surface interaction potentials. For a review of experiments see the CAMS (2005) proceedings.

\section{1. vdW-modified diffraction}

Because of van der Waals interactions with mechanical grating bars, atoms propagating through a nanograt-

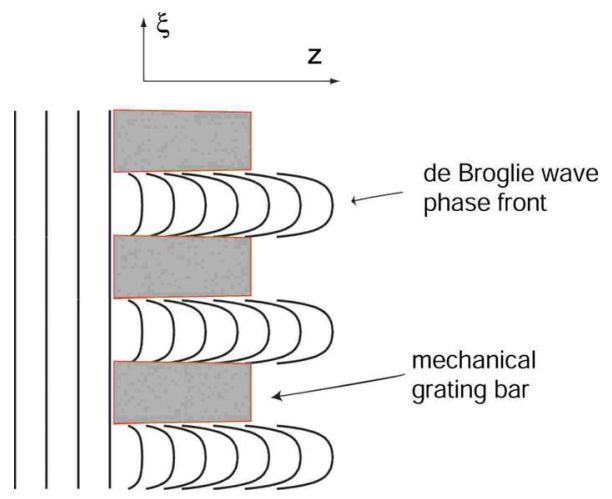

FIG. 63. (Color online) Distorted de Broglie waves. van der Waals interactions with mechanical grating bars cause nearfield phase shifts. This view is exaggerated: in beam experiments there are typically $10^{4}$ wave fronts in the 100 -nm thickness of a nanograting slot (Grisenti et al., 1999; Perreault et al., 2005).

ing get a phase shift that depends on position within each slot, as shown in Fig. 63. An analogous structure in light optics is an array of diverging lenses held between absorbing bars. The index of refraction in the free space between material grating bars gives nanogratings a complex transmission function that has been studied by Grisenti et al. (1999); Grisenti, Schöllkopf, Toennies, Manson, et al. (2000b); Shimizu (2001); Brezger et al. (2002); Bruehl et al. (2002); Kohno et al. (2003); Nairz et al. (2003); Cronin and Perreault (2004); Perreault and Cronin (2005, 2006); Perreault et al. (2005).

Figure 63 is a schematic of the de Broglie wave phase fronts in the near-field immediately after a nanograting. Far-field diffraction orders are affected by van der Waals interactions too. We can describe the $n$th far-field order by

$$
\psi_{n}=A_{n} e^{i \phi_{n}} e^{i \vec{k}_{n} \cdot \vec{x}},
$$

where the modulus $A_{n}$ and phase $\phi_{n}$ for the $n$th order are given by

$$
A_{n} e^{i \phi_{n}}=\int_{-w / 2}^{w / 2} \exp [i \phi(\xi)+i n G \xi] d \xi
$$

Here $w$ is the size of the windows (or "nanoslots") between grating bars and $\phi(\xi)$ is the phase shift in Fig. 63
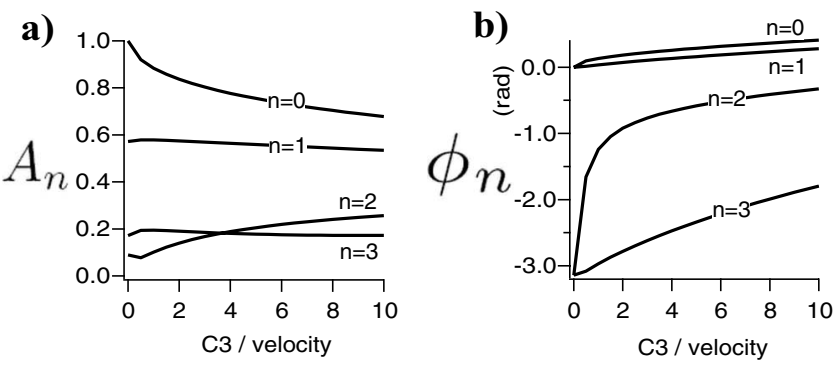

FIG. 64. The (a) phase (b) and modulus of far-field diffraction orders both depend on the vdW coefficient $C_{3}$ divided by atom velocity [shown in units of $\left.\mathrm{meV} \mathrm{nm}^{3} /(\mathrm{km} / \mathrm{s})\right]$. Adapted from Perreault and Cronin, 2006. 

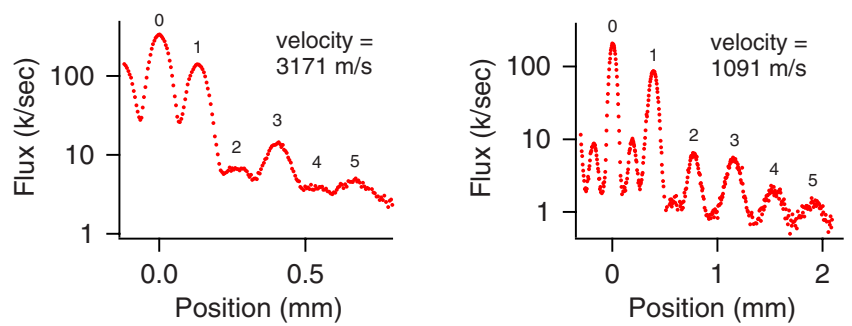

FIG. 65. (Color online) Diffraction intensities used to measure the strength of $C_{3}$ for Na-silicon nitride (Perreault et al., 2005). Data for two different velocities show how the second- and third-order change their relative intensity (as predicted in Fig. 64). Diffraction of $\mathrm{Na}_{2}$ molecules is also visible.

that can be calculated by putting the atom-surface potential $U(r)$ into the expression for a phase shift [Eq. (43)]. Thus the modulus and a phase of each diffraction order depends on the strength of the potential $\left(C_{3}\right.$ in the $\mathrm{vdW}$ regime) and on atomic velocity as shown in Fig. 64. Several experiments have measured the intensity $\left|A_{n}\right|^{2}$ in diffraction orders to determine $C_{3}$ for various atomsurface combinations, with some results shown in Fig. 65 and 66 (Grisenti et al., 1999; Bruehl et al., 2002; Cronin and Perreault, 2004; Perreault et al., 2005).

The diffraction intensities $\left|A_{n}\right|^{2}$ depend on phase gradients induced by $U(r)$. To detect the diffraction phases, $\phi_{n}$ an atom interferometer can be used as described in the next section.

\section{Interferometer vdW and CP measurements}

The complex transmission function of the gratings modifies the location at which the Talbot effect revivals occur. This, in turn, modifies the performance of a Talbot-Lau interferometer, as discussed by Brezger et al. (2002, 2003) and shown in Fig. 67. Because gratings in this experiment have a $1-\mu \mathrm{m}$ period, these results probe the retarded Casimir-Polder regime.

In a separated-path interferometer, Perreault and Cronin (2005) inserted an auxiliary interaction grating in one path. This allowed a measurement of the phase shift

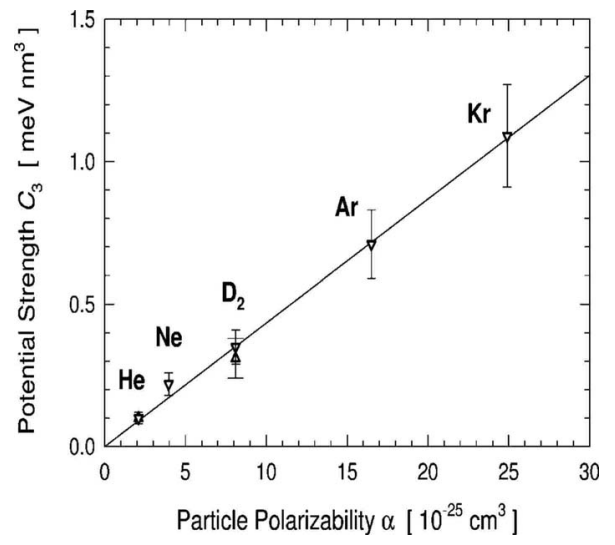

FIG. 66. Measurements of $C_{3}$ for various atoms and a silicon nitride surface, obtained by studying atom diffraction patterns. From Grisenti et al., 1999.

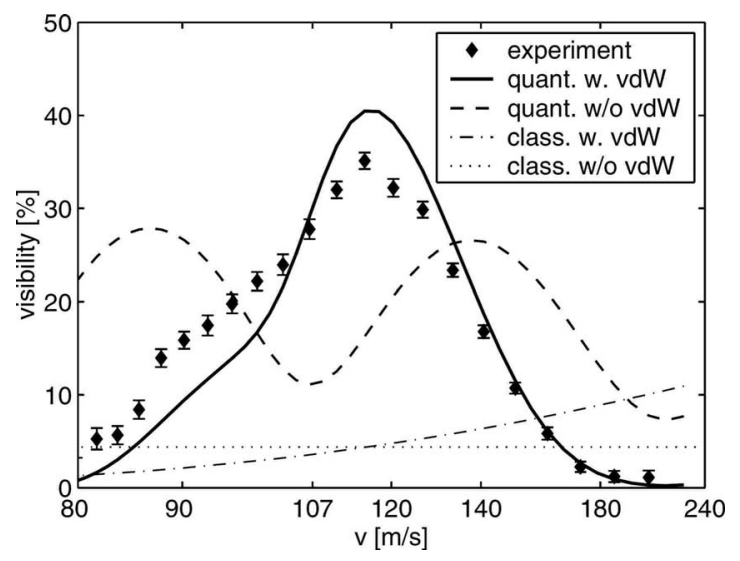

FIG. 67. Dependence of the interference fringe visibility on the mean velocity of the molecular beam. Numerical simulation results are plotted for four models without free parameters: classical or quantum behavior, with or without consideration of the van der Waals ( $\mathrm{vdW}$ ) interaction of the molecules with the second grating. The quantum result including the van der Waals effect is clearly the only adequate theory (Brezger et al., 2002).

$\phi_{0}$ due to transmission through the interaction grating as shown in Fig. 68. In a separate experiment the higherorder diffraction phase $\phi_{2}$ was measured by comparing the output of four different separated-path interferometers (Perreault and Cronin, 2006).

In the atomic beam spin-echo (ABSE) interferometer, discussed in Sec. III.D.2, Druzhinina and DeKieviet (2003) observed atoms reflecting from the attractive part of the atom-surface interaction potential. This quantum reflection allowed DeKieviet et al. to map the van der Waals potential in an energy range between $1 \mathrm{neV}$ and a sub-meV. Figure 69 shows the measured probability of $\mathrm{He}$ atoms quantum reflecting from a quartz surface as a function of the impinging wave vector. (Later both metallic and semiconductor samples were used.) Deviation of the experimental data from the high-energy asymptote is attributed to Casimir-Polder retardation. Importantly the spin-echo interferometer was used to precisely select the velocity of the detected atoms. In this regard it complements other quantum reflection experiments (Anderson et al., 1986; Berkhout et al., 1989; Shimizu, 2001; Shimizu and Fujita, 2002a; Pasquini et al., 2006)

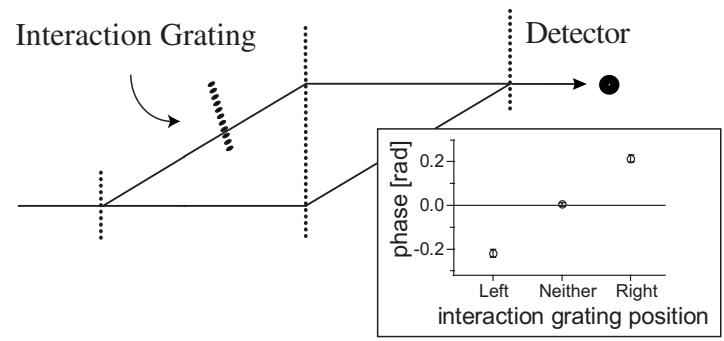

FIG. 68. An "interaction grating" was inserted and removed from each path of an interferometer to measure the phase shift $\Phi_{0}$ due to van der Waals interactions (Perreault and Cronin, 2005). 


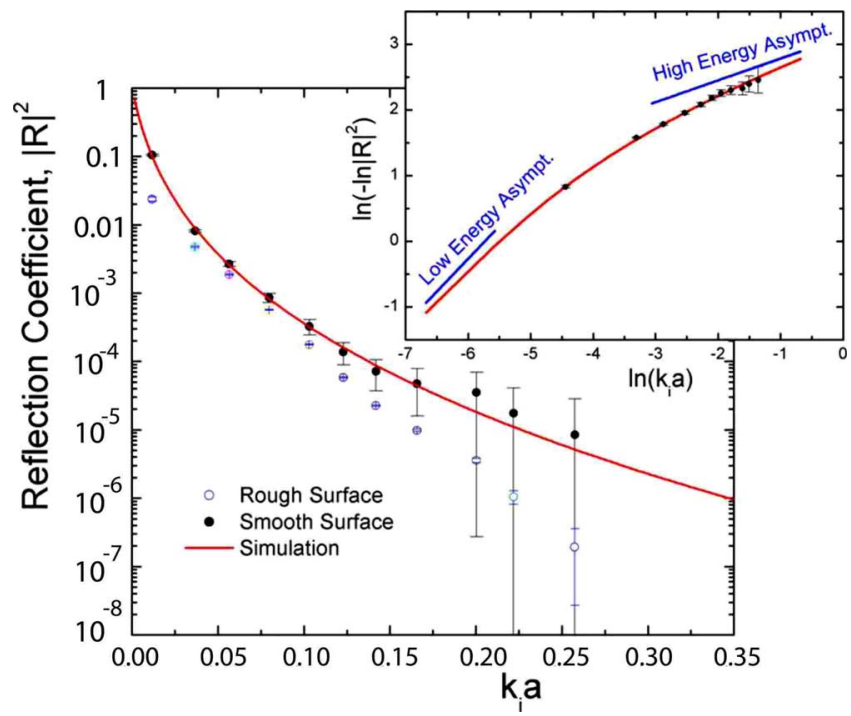

FIG. 69. (Color online) Experimental ABSE data for the quantum reflection of ${ }^{3} \mathrm{He}$ atoms from a disordered single crystal quartz surface. From Druzhinina and DeKieviet, 2003.

that do not explicitly use atom interferometers (though we note that quantum reflection itself is inherently a wave phenomenon).

\section{OUTLOOK}

In the early 1980s "mechanical effects of light" was the name for the study of light forces on atoms; see, for example, Chebotayev et al. (1985) and Table V. At first these forces were used simply to change the momentum of atoms. Then it emerged that, with care in application, light forces could be conservative. When atom diffraction from a standing light wave was demonstrated (Gould et al., 1986), it became appreciated that interactions with classical light fields can transfer momentum in precise quanta and preserve the coherence of atomic de Broglie waves. This led to many papers contrasting "diffraction and diffusion" and emphasizing that diffraction of atoms by light was coherent, whereas the occurrence of some spontaneous decay processes led to diffusion which is not coherent (Tanguy et al., 1983; Gould et al., 1991; Wilkens et al., 1991; Ryytty et al., 1998; Deng 2006). It also led workers in the field to consider other coherent ways to manipulate atoms. The term "optics" started to replace "diffraction" in conversations. Although some felt that "atomic optics" was the preferable phrase (in part to emphasize that atomic physics was the driving force), we felt that atom optics was more closely analogous to "electron optics" and decided to make it the title of our 1991 review in ICAP12 (Pritchard, 1991).

The reviews on atom optics that the MIT group wrote in 1990 and 1991 considered atom optics as a way to mimic photon optics. Relative to a list of standard optical components, it was pointed out that atom lenses could be made in various ways but that material beam splitters were impossible, shifting the burden for coherent beam splitting and recombining to diffractive pro-
TABLE V. Selected books, special journal issues, and review articles germane to atom interferometers.

Books

Atom Interferometry (Berman, 1997)

Atom Optics (Meystre, 2001)

Laser Cooling and Trapping (Metcalf and van der Stratten, 1999)

Neutron Interferometry (Rauch and Werner, 2000)

Electron Interferometry (Tonomura, 1999; Tonomura et al., 1999)

Molecular Beams (Ramsey, 1985)

Atomic and Molecular Beam Methods (Scoles, 1988)

Atomic, Molecular, and Optical Physics Handbook (Drake, 1996)

Atom and Molecular Beams, State of the Art (Campargue, 2000)

Encyclopedia of Modern Optics (Robert and Bayvel, 2004)

Special journal issues

JOSA-B: Mechanical effects of light (1985)

JOSA-B: Mechanical effects of light (1989)

JOSA-B: Atom Optics (1992)

Applied Physics B 54 (1992)

JOSA-B: Atom Optics (1994)

Journal de Physique: Optics and Interferometry with Atoms 4 (11) (1994)

Journal of Modern Optics: Quantum State Preparation and Measurement 44 (1997)

Comptes Rendus de L'Academie des sciences Dossier on

BEC and atom lasers, t.2 serie IV, (2001)

General Relativity and Gravitation, 36 (10) (2004)

Insight Review Articles in Nature 416 (2002)

Applied Physics B: Quantum Mechanics for Space

Application 84 (4) (2006)

Selected review articles

Atom optics (Pritchard, 1991)

Atom interferometry (Schmiedmayer et al., 1993)

The Feynman path-integral approach to atomic interferometry-A tutorial (Storey and Cohen-Tannoudji, 1994)

Atom optics (Adams et al., 1994)

Atom interferometry (Carnal and Mlynek, 1996)

de Broglie optics (Wilkens, 1996)

Precision atom interferometry (Peters et al., 1997)

Matter-wave index of refraction, inertial sensing, and quantum decoherence in an at. interf. (Hammond et al., 1997)

Interferometry with atoms and molecules: A tutorial

(Pritchard et al., 1997)

Atomic interferometry (Baudon et al., 1999)

Prospects for atom interferometry (Godun et al., 2001)

Atom optics: Old ideas, current technology, and new results (Pritchard et al., 2001) 
TABLE V. (Continued.)

Miniaturizing atom optics: from wires to atom chips

(Schmeidmayer and Folman, 2001)

Coherence with atoms (Kasevich, 2002)

Microscopic atom optics: From wires to an atom chip (Folman et al., 2002)

Atom interferometry (Miffre et al., 2006a)

For nonlinear atom optics

Nonlinear and quantum atom optics (Rolston and Phillips, 2002)

Nonlinear atom optics (Anderson and Meystre, 2003)

Physics with coherent matter waves (Bongs and Sengstock, 2004)

cesses using matter and light gratings. The observation that light and matter diffraction gratings would be the beam splitters has been borne out by the vast majority of work with atom interferometers over the past 15 years. However, their refinement has been quite remarkable. In addition, a host of new developments in atom optics have lengthened the list of atom optical components and devices; see the Atom Optics Toolkit in Table VI. The art of atom optics is in its golden age because the techniques listed in this toolkit are just beginning to have an impact on scientific questions beyond the specialty of atom optics. As larger and more controlled atom optical systems are constructed, opportunities abound to efficiently and coherently manipulate atoms for scientific gain.

Not all predictions by Pritchard (1991) were so prescient, however; although coherent atom amplifiers were discussed, they were not anticipated. Hence the demonstration of coherent atom amplification (using interferometry to verify its phase coherence) was an unexpected development, as was nonlinear atom optics generally. The power of, and interest in, nonlinear atom optics should lead to many more advances in atom interferometry such as sub-shot-noise measurements of phase shifts (Scully and Dowling, 1993; Search and Meystre, 2003; Pezze and Smerzi, 2006; Jo et al., 2007) and coherent oscillations between atomic and molecular BECs. Nonlinear optics is outside the scope of this review although techniques of linear atom optics and interferometry are extremely valuable as tools in this field (Meystre, 2001; Rolston and Phillips, 2002; Anderson and Meystre, 2003; Bongs and Sengstock, 2004). Another unanticipated development is the immense amount of development on atom chips.

As this review shows, experimental and theoretical understanding of atomic and molecular matter waves has come a long way since the first demonstrations of coherent diffraction with laser light and nanogratings in the early 1990s. In the MIT group's first paper on diffraction by a light grating, the rms momentum transfer was far below predictions; the second paper reported it was low by a factor of 2 noting there was "no explanation for this discrepancy." Recently this effect was used to measure the standing wave intensity of a standing wave (depth of optical lattice) to within 1\% (Mun et al., 2007).

This shows the transformation of pioneering scientific work in atom optics into a high-precision tool for use in cold atom physics. Similarly, our review shows that atom interferometers are now routinely used for scientific endeavors ranging from fundamental investigations of quantum physics to precision metrology. We now project anticipated progress over the main categories used in this review (diffraction, interferometry, fundamental studies, precision measurements, and atomic properties). We also speculate on areas that we expect will become more important: e.g., optics with molecules and ultracold fermions, atom chips and optical lattices, surface science, fundamental studies of gravitation, new ways to control atom-atom interactions, entanglement and multiparticle interferometry, and more formal analogies to condensed matter phenomena that arise from quantum coherence.

We expect coherent atom optics to become an even more flexible, powerful, and precise tool for manipulating atoms and molecules, especially for interferometers, and for applications to other scientific and technical problems. The development of techniques for accelerating (and in the future decelerating) atoms and especially molecules, both in light crystals and by optimizing the temporal envelope of light for higher-order beam splitters will enable coherence to be maintained between wave function components with relative velocities of $\mathrm{m} / \mathrm{s}$ that are determined with $10^{-10}$ accuracy. This will result in interferometers of far greater precision with much greater separation of the arms and much greater enclosed area.

These bigger and better interferometers will be applied to fundamental problems in gravity and quantum mechanics. They will allow one to measure the gravitational potential in experiments analogous to the scalar Aharonov-Bohm effect in which the potential has influence in the absence of any gravitational field (such as when one component of the wave function spends time inside a hollow massive cylinder). Placed in orbit around the Earth, interferometers with large enclosed area will be useful for fundamental gravitational measurements such as tests of parallel vector transport and the LenseThirring frame-dragging effect. As a by-product of developing interferometers with larger separation for heavier particles, more stringent limits will placed on alternative theories of quantum decoherence that involve spontaneous projection. It may also be possible to observe some new sources of decoherence that are hard to shield out (Tegmark, 1993). These advances in interferometer size will also enable better measurements of inertial effects such as gravitational fields, gravitational gradients, and in gyroscopes. These will have application to inertial navigation, geodesy, and prospecting.

Precision in atom and molecular interference experiments will also be increased using higher fluxes and longer interaction times. This also implies larger instruments in order to reduce the atom densities, thus reduc- 
TABLE VI. Atom optics tool kit organized by analogy to light optics, as in Pritchard (1991). Parentheses indicate options.

\begin{tabular}{|c|c|c|}
\hline & Light optics & Atom optics \\
\hline \multirow[t]{3}{*}{ Sources } & thermal & (supersonic) beam \\
\hline & & $\begin{array}{l}\text { (moving) molasses, launched (or dropped) } \\
\text { MOT, Zeeman slower }\end{array}$ \\
\hline & coherent laser & Bose-Einstein condensate \\
\hline \multirow[t]{5}{*}{ Lenses } & spherical & electrostatic quadrupole or magnetic hexapole \\
\hline & cylindrical & Gaussian optical beams \\
\hline & Fresnel & nanostructure zone plates (cyl. or sph.) \\
\hline & achromat & combination zone plate $+E-M$ lens \\
\hline & axicon & magnetic quadrupole \\
\hline \multirow[t]{4}{*}{ Mirrors } & & (giant) quantum reflection \\
\hline & & helium from (bent) crystal surfaces \\
\hline & & evanescent light waves \\
\hline & & $\begin{array}{l}\text { periodically poled magnetic domains (on } \\
\text { curved surfaces) }\end{array}$ \\
\hline \multirow[t]{8}{*}{ Gratings } & phase & $\begin{array}{l}\text { standing waves of light: Bragg or } \\
\text { Kapitza-Dirac (pulses) }\end{array}$ \\
\hline & amplitude & nanostructure gratings \\
\hline & & standing waves of resonant radiation \\
\hline & reflection & crystal surfaces \\
\hline & & $\begin{array}{l}\text { quantum reflection from (nanostructured) } \\
\text { surfaces }\end{array}$ \\
\hline & & structured evanescent light \\
\hline & blazed & Bragg scattering \\
\hline & & two- and three-color standing waves \\
\hline \multirow[t]{4}{*}{ Polarizing splitters } & & stimulated Raman transitions \\
\hline & & optical Ramsey $\pi / 2$ pulses \\
\hline & & Stern-Gerlach magnets \\
\hline & & optical Stern-Gerlach effect \\
\hline \multirow[t]{4}{*}{ Phase plates } & glass & E field \\
\hline & & B field \\
\hline & & dilute gas \\
\hline & & nearby surface \\
\hline \multirow[t]{2}{*}{ Holograms } & transmission & perforated nanostructures (with $\mathbf{E}$ and $\mathbf{B}$ fields) \\
\hline & reflection & $\begin{array}{l}\text { nanostructures (with enhanced quantum } \\
\text { reflection) }\end{array}$ \\
\hline \multirow[t]{4}{*}{$\lambda$ shifters } & modulators & amplitude modulated standing waves \\
\hline & & gravity \\
\hline & & bichromatic laser fields \\
\hline & & reflection from a receding rotor \\
\hline \multirow[t]{7}{*}{ Interferometers } & Young's experiment & micro (or nano) slits \\
\hline & Mach-Zehnder & $\begin{array}{l}\text { space domain using (separated) beams (spin } \\
\text { entanglement) }\end{array}$ \\
\hline & & $\begin{array}{l}\text { time domain, with pulsed gratings (spin } \\
\text { entanglement) }\end{array}$ \\
\hline & & $\begin{array}{l}\text { longitudinal (rf or Stern-Gerlach beam } \\
\text { splitters) }\end{array}$ \\
\hline & near field & Talbot Lau, Lau, and Talbot interferometers \\
\hline & Michelson & atoms confined in a waveguide \\
\hline & Fabry-Perot & atoms confined in a three-dimensional trap \\
\hline Wave guides & fiber optics & B fields from wires (on a chip) \\
\hline
\end{tabular}


TABLE VI. (Continued.)

\begin{tabular}{|c|c|c|}
\hline & Light optics & Atom optics \\
\hline & & permanent magnets \\
\hline & & optical dipole force \\
\hline & & evanescent light in hollow fiber \\
\hline \multirow[t]{4}{*}{ Detectors } & photon counter & $\begin{array}{l}\text { hot wire (or electron bombardment) ionizer and } \\
\text { counter (CEM or MPC) }\end{array}$ \\
\hline & state selective & $\begin{array}{l}\text { field ionization, laser ionization, metastable } \\
\text { detection }\end{array}$ \\
\hline & & polarization spectroscopy \\
\hline & imaging & $\begin{array}{l}\text { multichannel plate for ions or (metastable) atoms } \\
\text { (state selective) fluorescence, absorption, or phase } \\
\text { contrast imaging }\end{array}$ \\
\hline Amplifiers & stimulated emission & $\begin{array}{l}\text { four-wave mixing with BEC (nonlinear quantum } \\
\text { optics) }\end{array}$ \\
\hline
\end{tabular}

ing the systematic shifts due to atom-atom interactions. However, more imaginative approaches are needed since atom-atom interactions can be a severe problem. For example, they are one of the limiting factors for the Cs atomic fountain clocks, all interferometers using Bose-Einstein condensates, and they modify the index of refraction of near-resonant light passing through even nondegenerate atom samples. There are at least two solutions to the problem:

- If one uses ultracold fermions in a single atomic state, the Pauli exclusion principle switches off the $s$-wave interaction. Since for neutral atoms at ultracold energies the higher partial waves can be ignored, a fermionic ensemble is nearly interaction free, and therefore ideal for precision measurements. This was demonstrated in the Bloch oscillation experiment by Pezze et al. (2004).

- The second solution is to put each atom in a separate potential well, for example, in an optical lattice. Having only one atom per well reduces the nonlinear interaction. The effects of these additional potential wells can be mitigated using light that energy shifts the interfering states equally.

Application of atom interferometers to atomic and molecular physics will benefit from advances in precision and should continue to provide definitive measurements with higher precision. A key application will be determination of polarizabilities and Stark shifts for atoms and molecules in applied fields. These will serve as benchmark measurements to test and refine atomic theory calculations as discussed in Sec. V.

Since atoms are very small, techniques for their manipulation on small scales will open up many scientific frontiers and technical possibilities in surface physics, nanophysics, and quantum information. The rapid pace of current developments in atom chips and the more creative use of focused light beams and light crystals are both leading to techniques for producing, detecting, and coherently manipulating atoms on very small spatial scales, e.g., where tunneling can be carefully studied.

Small interferometers will enable novel applications in surface science. Atom interferometry can be used to (i) measure fundamental atom-surface interactions like the van der Waals and Casimir potentials or (ii) study the temporal and spatial behavior of electromagnetic fields close to the surface. This will allow new probes of surface structure-both magnetic and electric. The nature of thermally induced time varying fields can be studied, both for its own sake and because such fields induce decoherence. This will lead to engineering advances that reduce deleterious decoherence close to surfaces, advancing quantum information technology that uses ions and atoms close to surfaces as $q$-bits.

Coherent atom optics generally, and interferometers in particular, will be applicable to a central problem in quantum information science: how to characterize, control, and use entanglement and correlations in atomic ensembles. The challenge here will be to prepare the ensembles in complex quantum states with high fidelity, and to develop methods for their characterization-with decoherence reduced as much as possible (or with its effects reduced by error-correction methods). One helpful new interferometric technique will be the development of powerful homodyne and heterodyne methods for detecting atoms, in analogy to quantum optics. This will be greatly aided by the development of detection methods with high quantum efficiency, which are also highly desirable in studying atom-atom correlations, particularly of higher order.

Having a good understanding of the electromagnetic atom-surface interaction, and ways to mitigate nearsurface decoherence, the physics community will have a tool to search for fundamental short-range interactions, as predicted in some unified theories. In principle atom interferometry has the potential to improve the present limits on non-Newtonian gravitational potentials at the micrometer length scale by many orders of magnitude (Dimopoulos and Geraci, 2003). The main challenge here will be to control the systematic effects, mainly 
coming from the electromagnetic interactions of the atom with close-by surfaces, and the atom-atom interactions as discussed below. Smaller and more compact atom interferometers also have application to inertial sensors for commercial applications.

Atom interference will be one of the central tools in the study of many-atom systems generally and of atoms in lattices that model condensed matter Hamiltonians in particular. First, diffraction peaks are the hallmark of atoms in the regime where tunneling dominates in a periodic lattice (Bloch et al., 2000). As more complex lattices are studied, higher-order interference will play a role. In their turn, these lattices can have regions where a particular number of atoms are confined in each lattice site; this suggests a way to make a source of atomic number states allowing studies of degenerate atomic systems. Especially interesting in this arena will be the study of phase transitions in mesoscopic ensembles, which are too large to permit full quantum calculations, but too small for the thermodynamic description to be valid. This will give us a new and detailed look at the thermodynamic border. There are many new avenues to explore with dense degenerate quantum gases. In the present review we focused on single particle interference or, in the language of quantum optics, to first-order coherence. One fruitful avenue will be extension to multiparticle interferometry, which can give more rapidly varying fringes and sub-shot-noise statistical precision. Detecting higher-order coherence requires measurements of correlations between $\mathrm{N}$ atoms. Noise correlation with bosons and fermions (Yasuda and Shimizu, 1996; Altman et al., 2004; Schellekens et al., 2005; Gritsev et al., 2006; Hofferberth et al., 2006; Morsch and Oberthaler, 2006; Polkovnikov et al., 2006; Hofferberth, Lesanovsky, Schumm, et al., 2007; Jeltes et al., 2007) are examples of recent developments in this field.

The field of atom and molecular interference is young but has already impacted atomic and quantum physics across a broad frontier. New techniques and the application of previously developed techniques to new scientific problems promises much future scientific gain.

\section{ACKNOWLEDGMENTS}

We thank the many research leaders who provided references and helpful material to make this review more complete. We received a tremendous number of contributions and could only include a selection because of the length requirements. A.D.C. and D.E.P. acknowledge support from National Science Foundation Grants No. PHY-0653623 and No. PHY-0457451, respectively.

\section{REFERENCES}

Abfalterer, R., C. Keller, S. Bernet, M. K. Oberthaler, J. Schmiedmayer, and A. Zeilinger, 1997, "Nanometer definition of atomic beams with masks of light," Phys. Rev. A 56, R4365.

Abraham, M., 1909, “Zur elektrodynamik bewegter Körper,” Rend. Circ. Mat. Palermo 28, 1.
Adams, C., M. Sigel, and J. Mlynek, 1994, "Atom optics," Phys. Rep. 240, 143.

Aharonov, Y., and J. Anandan, 1987, "Phase-change during a cyclic quantum evolution," Phys. Rev. Lett. 58, 1593.

Aharonov, Y., and D. Bohm, 1959, "Significance of electromagnetic potentials in the quantum theory," Phys. Rev. 115, 485.

Aharonov, Y., and A. Casher, 1984, "Topological quantum effects for neutral particles," Phys. Rev. Lett. 53, 319.

Aharonov, Y., P. Pearle, and L. Vaidman, 1988, "Comment on 'Proposed Aharonov-Casher effect: Another example of an Aharonov-Bohm effect arising from a classical lag," "Phys. Rev. A 37, 4052.

Albiez, M., R. Gati, J. Folling, S. Hunsmann, M. Cristiani, and M. K. Oberthaler, 2005, "Direct observation of tunneling and nonlinear self-trapping in a single bosonic Josephson junction," Phys. Rev. Lett. 95, 010402.

Allis, R., P. Gettings, and D. Chapman, 2000, "Precise gravimetry and geothermal reservoir management," Proceedings of the 25th Workshop on Geothermal Reservoir Engineering SGP-TR-165 (Stanford Geothermal Program, Stanford, CA). Allman, B. E., A. Cimmino, A. G. Klein, G. I. Opat, H. Kaiser, and S. A. Werner, 1992, "Scalar Aharonov-Bohm experiment with neutrons," Phys. Rev. Lett. 68, 2409.

Altman, E., E. Demler, and M. D. Lukin, 2004, "Probing many-body states of ultra-cold atoms via noise correlations," Phys. Rev. A 70, 013603.

Altschuler, S., and L. M. Franz, 1973, "Matter wave interferometric apparatus," U.S. Patent No. 3,761,721.

Anandan, J., 1981, "Sagnac effect in relativistic and nonrelativistic physics," Phys. Rev. D 24, 338.

Anandan, J., 1989, "Electromagnetic effects in the quantum interference of dipoles," Phys. Lett. A 138, 347.

Anandan, J., 2000, "Classical and quantum interaction of the dipole,” Phys. Rev. Lett. 85, 1354.

Anandan, J., J. Christian, and K. Wanelik, 1997, "Resource letter GPP-1: Geometric phases in physics," Am. J. Phys. 65, 180.

Anderson, A., S. Haroche, E. A. Hinds, W. Jhe, and D. Meschede, 1988, "Measureing the van der waals forces between a Rydberg atom and a metallic surface," Phys. Rev. A 37, 3594.

Anderson, A., S. Haroche, E. A. Hinds, W. Jhe, D. Meschede, and L. Moi, 1986, "Reflection of thermal Cs atoms grazing a polished galss surface," Phys. Rev. A 34, 3513.

Anderson, B., and P. Meystre, 2003, "Nonlinear atom optics," Contemp. Phys. 44, 473.

Anderson, B. P., and M. A. Kasevich, 1998, "Macroscopic quantum interference from atomic tunnel arrays," Science 282, 1686 .

Anderson, M. H., J. R. Ensher, M. R. Matthews, C. E. Wieman, and E. A. Cornell, 1995, "Observation of Bose-Einstein condensation in a dilute atomic vapor," Science 269, 198.

Andersson, E., T. Calarco, R. Folman, M. Andersson, B. Hessmo, and J. Schmiedmayer, 2002, "Multimode interferometer for guided matter waves," Phys. Rev. Lett. 88, 100401.

Andrews, M. R., C. Townsend, H. Miesner, D. Durfee, D. Kurn, and W. Ketterle, 1997, "Observation of interference between two bose condensates," Science 275, 637.

Aoki, T., M. Yasuhara, and A. Morinaga, 2003, "Atomic multiple-wave interferometer phase-shifted by the scalar Aharonov-Bohm effect," Phys. Rev. A 67, 053602.

Arimondo, E., H. Lew, and T. Oka, 1979, "Deflection of a Na 
beam by resonant standing-wave radiation," Phys. Rev. Lett. 43, 753.

Arndt, M., K. Hornberger, and A. Zeillinger, 2005, "Probing the limits of the quantum world," Phys. World 18, 35.

Arndt, M., O. Nairz, J. Petschinka, and A. Zeilinger, 2001, "High contrast interference with C-60 and C-70," C. R. Acad. Sci., Ser IV: Phys., Astrophys. 2, 581.

Arndt, M., O. Nairz, J. VosAndreae, C. Keller, G. Van der Zouw, and A. Zeilinger, 1999, "Wave-particle duality of C-60 molecules," Nature (London) 401, 680.

Arndt, M., P. Szriftgiser, J. Dalibard, and A. M. Steane, 1996, "Atom optics in the time domain," Phys. Rev. A 53, 3369.

Ashkin, A., 1970, "Acceleration and trapping of particles by radiation pressure," Phys. Rev. Lett. 24, 156.

Ashkin, A., 1980, "Applications of laser-radiation pressure," Science 210, 1081.

Aspect, A., E. Arimondo, R. Kaiser, N. Vansteenkiste, and C. Cohen-Tannoudji, 1988, "Laser cooling below the onephoton recoil energy by velocity-selective coherent population trapping," Phys. Rev. Lett. 61, 826.

Aspect, A., J. Dalibard, A. Heidmann, C. Salomon, and C. Cohen-Tannoudji, 1986, "Cooling atoms with stimulatedemission," Phys. Rev. Lett. 57, 1688.

Audouard, E., P. Duplaa, and J. Vigué, 1995, "Glory and resonance effects in the index of refraction for atomic waves," Europhys. Lett. 32, 397.

Audouard, E., P. Duplaa, and J. Vigué, 1997, "Erratum: Glory and resonance effects in the index of refraction for atomic waves," Europhys. Lett. 37, 311.

Audretsch, J., and V. D. Skarzhinsky, 1998, "Aharonov-Bohm scattering of neutral atoms with induced electric dipole moments," Phys. Lett. A 241, 7.

Badurek, G., and H. Rauch, 2000, "Neutron interferometry," Physica B 276-278, 964.

Badurek, G., H. Rauch, and J. Summhammer, 1983, "Timedependent superposition of spinors," Phys. Rev. Lett. 51, 1015.

Badurek, G., H. Rauch, and J. Summhammer, 1988, "Polarized neutron interferometry: A survey," Physica B \& C 151, 82.

Badurek, G., H. Weinfurter, R. Gahler, A. Kollmar, S. Wehinger, and A. Zeilinger, 1993, "Nondispersive phase of the Aharonov-Bohm effect," Phys. Rev. Lett. 71, 307.

Bagnato, V., L. Mardassa, C. Tsao, Y. Wang, and J. Weiner, 1993, "Two-color spectroscopy of colliding ultracold atoms," Phys. Rev. Lett. 70, 3225.

Bagnato, V., D. Pritchard, and D. Kleppner, 1987, "BoseEinstein condensation in an external potential," Phys. Rev. A 35, 4354

Bartelt, H. O., and J. Jahns, 1979, "Interferometry based on the Lau effect," Opt. Commun. 30, 268.

Batelaan, H., 2000, "The Kapitza-Dirac effect," Contemp. Phys. 41, 369.

Batelaan, H., S. Barnet, M. K. Oberthaler, E. Rasel, J. Schmiedmayer, and A. Zeilinger, 1997, "Classical and quantum atoms fringes," in Atom Interferometry, edited by P. R. Berrman (Academic Press, San Diego), pp. 85-120.

Battesti, R., P. Clade, S. Guellati-Khelifa, C. Schwob, B. Gremaud, F. Nez, L. Julien, and F. Biraben, 2004, "Bloch oscillations of ultracold atoms: A tool for a metrological determination of $h / m_{\mathrm{Rb}}$," Phys. Rev. Lett. 92, 253001.

Baudon, J., R. Mathevet, and J. Robert, 1999, "Atomic interferometry," J. Phys. B 32, R173.

Baumann, J., R. Gahler, J. Kalus, and W. Mampe, 1988, "Ex- perimental limit for the charge of the free neutron," Phys. Rev. D 37, 3107.

Baumann, P., D. Zimmermann, and R. Bruehl, 1992, "Laser spectroscopic investigation of the van der waals molecule NaXe," J. Mol. Spectrosc. 155, 277.

Beijerinck, H. C. W., and N. F. Verster, 1981, “Absolute intensities and perpendicular temperatures of supersonic beams of polyatomic gases," Physica B \& C 111, 327.

Bell, A. S., B. Brezger, U. Drodofsky, S. Nowak, T. Pfau, J. Stuhler, T. Schulze, and J. Mlynek, 1999, "Nano-lithography with atoms," Surf. Sci. 433-435, 40.

Bergmann, K., H. Theuer, and B. W. Shore, 1998, "Coherent population transfer among quantum states of atoms and molecules," Rev. Mod. Phys. 70, 1003.

Berkhout, J. J., O. J. Luiten, I. D. Setija, T. W. Hijmans, T. Mizusaki, and J. T. M. Walraven, 1989, "Quantum reflection-Focusing of hydrogen-atoms with a concave mirror," Phys. Rev. Lett. 63, 1689.

Berman, P., 1997, Ed., Atom Interferometry (Academic Press, San Diego).

Bernet, S., R. Abfalterer, C. Keller, M. K. Oberthaler, J. Schmiedmayer, and A. Zeilinger, 2000, "Matter waves in time-modulated complex light potentials," Phys. Rev. A 62, 023606.

Bernet, S., R. Abfalterer, C. Keller, J. Schmiedmayer, and A. Zeilinger, 1999, "Matter wave sidebands from a complex potential with temporal helicity," Proc. R. Soc. London, Ser. A 455, 1509.

Bernet, S., M. K. Oberthaler, R. Abfalterer, J. Schmiedmayer, and A. Zeilinger, 1996, "Coherent frequency shift of atomic matter waves," Phys. Rev. Lett. 77, 5160.

Berninger, M., A. Stefanov, S. Deachapunya, and M. Arndt, 2007, "Polarizability measurements of a molecule via a nearfield matter-wave interferometer," Phys. Rev. A 76, 013607.

Berry, M. V., 1984, "Quantal phase factors accompanying adiabatic changes," Proc. R. Soc. London, Ser. A 392, 45.

Bertet, P., S. Osnaghi, A. Rauschenbeutel, G. Nogues, A. Auffeves, M. Brune, J. M. Raimond, and S. Haroche, 2001, "A complementarity experiment with an interferometer at the quantum-classical boundary," Nature (London) 411, 166. Bertoldi, A., G. Lamporesi, L. Cacciapuoti, M. de Angelis, M. Fattori, T. Petelski, A. Peters, M. Prevedelli, J. Stuhler, and G. M. Tino, 2006, “Atom interferometry gravity-gradiometer for the determination of the Newtonian gravitational constant G," Eur. Phys. J. D 40, 271.

Bitter, T., and D. Dubbers, 1987, "Manifestation of Berry topological phase in neutron spin rotation," Phys. Rev. Lett. 59, 251.

Blakie, P. B., and R. J. Ballagh, 2000, "Mean-field treatment of Bragg scattering from a Bose-Einstein condensate," J. Phys. B 33, 3961.

Blanchard, S., D. Civello, and R. C. Forrey, 2003, "Index of refraction for sodium matter waves traveling in a cold noblegas medium," Phys. Rev. A 67, 013604.

Bloch, F., 1929, "Uber die quantenmechanik der elektronen in kristallgittern," Z. Phys. 52, 555.

Bloch, I., T. Hansch, and T. Esslinger, 2000, "Measurement of the spatial coherence of a trapped Bose gas at the phase transition," Nature (London) 403, 166.

Bloch, I., T. W. Hansch, and T. Esslinger, 1999, "Atom laser with a cw output coupler," Phys. Rev. Lett. 82, 3008.

Bohr, N., 1949, A. Einstein: Philosopher-Scientist, Library of Living Philosophers (Academic Press, Evaston), see quote 
from N. Bohr, pp. 200-241.

Bongs, K., R. Launay, and M. A. Kasevich, 2006, "High-order inertial phase shifts for time-domain atom interferometers," Appl. Phys. B: Lasers Opt. 84, 599.

Bongs, K., and K. Sengstock, 2004, "Physics with coherent matter waves," Rep. Prog. Phys. 67, 907.

Bonin, K., and M. Kadarkallen, 1994, "Linear electric-dipole polarizabilities,” Int. J. Mod. Phys. B 8, 3313.

Bonse, U., and H. Rauch, 1979, Neutron Interferometry (Oxford University Press, Oxford).

Bordé, C., N. Courtier, F. duBurck, A. Goncharov, and M. Gorlicki, 1994, "Molecular interferometry experiments," Phys. Lett. A 188, 187.

Bordé, C. J., 1989, "Atomic interferometry with internal state labelling," Phys. Lett. A 140, 10.

Bordé, C. J., 1995, "Amplification of atomic fields by stimulated-emission of atoms," Phys. Lett. A 204, 217.

Bordé, C. J., 1997, "Matter-wave interferometers: a synthetic approach," in Atom Interferometry, edited by P. R. Berrman (Academic Press, San Diego), pp. 257-292.

Borrmann, G., 1941, "The absorbance diagram of quartz," Phys. Z. 42, 157.

Boyer, T. H., 1987, "Proposed Aharonov-Casher effect: Another example of an Aharonov-Bohm effect arising from a classical lag," Phys. Rev. A 36, 5083.

Bradley, M. P., J. V. Porto, S. Rainville, J. K. Thompson, and D. E. Pritchard, 1999, "Penning trap measurements of the masses of ${ }^{133} \mathrm{Cs},{ }^{87} \mathrm{Rb},{ }^{85} \mathrm{Rb}$, and ${ }^{23} \mathrm{Na}$ with uncertainties $\leqslant 0.2$ ppb," Phys. Rev. Lett. 83, 4510 .

Brezger, B., M. Arndt, and A. Zeilinger, 2003, "Concepts for near-field interferometers with large molecules," J. Opt. B: Quantum Semiclassical Opt. 5, S82.

Brezger, B., L. Hackermuller, S. Uttenthaler, J. Petschinka, M. Arndt, and A. Zeilinger, 2002, "Matter-wave interferometer for large molecules," Phys. Rev. Lett. 88, 100404.

Bruch, L. W., W. Schöllkopf, and J. P. Toennies, 2002, "The formation of dimers and trimers in free jet ${ }^{4} \mathrm{He}$ cryogenic expansions," J. Chem. Phys. 117, 1544.

Bruehl, R., P. Fouquet, R. E. Grisenti, J. P. Toennies, G. C. Hegerfeldt, T. Kohler, M. Stoll, and D. Walter, 2002, "The van der waals potential between metastable atoms and solid surfaces: Novel diffraction experiments vs theory," Europhys. Lett. 59, 357.

Bruehl, R., R. Guardiola, A. Kalinin, O. Kornilov, J. Navarro, T. Savas, and J. P. Toennies, 2004, "Diffraction of neutral helium clusters: Evidence for "magic numbers," " Phys. Rev. Lett. 92, 185301.

Bruehl, R., A. Kalinin, O. Kornilov, J. P. Toennies, G. C. Hegerfeldt, and M. Stoll, 2005, "Matter wave diffraction from an inclined transmission grating: Searching for the elusive ${ }^{4} \mathrm{He}$ trimer Efimov state," Phys. Rev. Lett. 95, 063002.

Bryant, H. C., and A. J. Cox, 1966, "Mie theory and the glory," J. Opt. Soc. Am. 56, 1529.

Buchner, M., R. Delhuille, A. Miffre, C. Robilliard, J. Vigué, and C. Champenois, 2003, "Diffraction phases in atom interferometers," Phys. Rev. A 68, 013607.

Burkov, A. A., M. D. Lukin, and E. Demler, 2007, "Decoherence dynamics in low-dimensional cold atoms interferometers," Phys. Rev. Lett. 98, 200404.

Cahn, S. B., A. Kumarakrishnan, U. Shim, T. Sleator, P. R. Berman, and B. Dubetsky, 1997, "Time-domain de Broglie wave interferometry," Phys. Rev. Lett. 79, 784.

Caldeira, A. O., and A. J. Leggett, 1983, "Path integral ap- proach to quantum brownian-motion," Physica A 121, 587. Calderbank, A. R., and P. W. Shor, 1996, "Good quantum error-correcting codes exist,” Phys. Rev. A 54, 1098.

Campargue, R., 1984, "Progress in overexpanded supersonic jets and skimmed molecular-beams in free-jet zones of silence," J. Phys. Chem. 88, 4466.

Campargue, R., 2000, Ed., Atomic and Molecular Beams, The State of the Art (Springer-Verlag, Berlin).

Campbell, G. K., A. E. Leanhardt, J. Mun, M. Boyd, E. W. Streed, W. Ketterle, and D. E. Pritchard, 2005, "Photon recoil momentum in dispersive media," Phys. Rev. Lett. 94, 170403. CAMS, 2005, "Conference on atoms and molecules near surfaces," J. Phys.: Conf. Ser. 19, 1.

Carnal, O., and J. Mlynek, 1991, "Young's double slit experiment with atoms: A simple atom interferometer," Phys. Rev. Lett. 66, 2689.

Carnal, O., and J. Mlynek, 1996, “Atom interferometry,” Exp. Methods Phys. Sci. 29B, 341.

Carnal, O., M. Sigel, T. Sleator, H. Takuma, and J. Mlynek, 1991, "Imaging and focusing of atoms by a Fresnel zone plate," Phys. Rev. Lett. 67, 3231.

Carusotto, I., M. Artoni, and G. C. La Rocca, 2000, "Atomlaser coherence length and atomic standing waves," Phys. Rev. A 62, 063606.

Casella, R. C., 1990, "Nonlocal phase-shifts induced by static electric-fields in neutron interferometers when the pathenclosed charge vanishes," Phys. Rev. Lett. 65, 2217.

Casimir, H. B. G., and D. Polder, 1948, "The influence of retardation on the London-van der Waals forces," Phys. Rev. 73, 360 .

Cassettari, D., A. Chenet, R. Folman, A. Haase, B. Hessmo, P. Kruger, T. Maier, S. Schneider, T. Calarco, and J. Schmiedmayer, 2000, "Micromanipulation of neutral atoms with nanofabricated structures," Appl. Phys. B: Lasers Opt. 70, 721.

Cassettari, D., B. Hessmo, R. Folman, T. Maier, and J. Schmiedmayer, 2000, "Beam splitter for guided atoms," Phys. Rev. Lett. 85, 5483.

Castin, Y., and J. Dalibard, 1997, "Relative phase of two BoseEinstein condensates," Phys. Rev. A 55, 4330.

Cataliotti, F. S., S. Burger, C. Fort, P. Maddaloni, F. Minardi, A. Trombettoni, A. Smerzi, and M. Inguscio, 2001, "Josephson junction arrays with Bose-Einstein condensates," Science 293, 843.

Cataliotti, F. S., L. Fallani, F. Ferlaino, C. Fort, P. Maddaloni, and M. Inguscio, 2003, "Superfluid current disruption in a chain of weakly coupled Bose-Einstein condensates," New J. Phys. 5, 71.

Chadan, K., and P. Sabatier, 1989, Inverse Problems in Quantum Scattering Theory (Springer, New York).

Champenois, C., E. Audouard, P. Duplaa, and J. Vigué, 1997, "Refractive index for atomic waves: Theory and detailed calculations," J. Phys. II 7, 523.

Champenois, C., M. Buchner, R. Dehuille, R. Mathevet, C. Robillard, C. Rizzo, and J. Vigué, 2001a, "Atomic diffraction by a laser standing wave: Analysis using Bloch states," Eur. Phys. J. D 13, 271.

Champenois, C., M. Buchner, R. Dehuille, R. Mathevet, C. Robillard, C. Rizzo, and J. Vigué, 2001b, Matter Neutrality Test Using a Mach-Zehnder Interferometer, Lecture Notes in Physics Vol. 570 (Springer, New York), p. 554.

Chapman, M., C. Ekstrom, T. Hammond, R. Rubenstein, J. Schmiedmayer, S. Wehinger, and D. Pritchard, 1995, "Optics 
and interferometry with $\mathrm{Na}_{2}$ molecules," Phys. Rev. Lett. 74, 4783.

Chapman, M., T. D. Hammond, A. Lenef, J. Schmiedmayer, R. R. Rubenstein, E. Smith, and D. E. Pritchard, 1995, "Photon scattering from atoms in an atom interferometer: Coherence lost and regained," Phys. Rev. Lett. 75, 3783.

Chapman, M. S., C. R. Ekstrom, T. D. Hammond, J. Schmiedmayer, B. E. Tannian, S. Wehinger, and D. E. Pritchard, 1995, "Near field imaging of atom diffraction gratings: The atomic Talbot effect," Phys. Rev. A 51, R14.

Chebotayev, V., B. Dubetsky, A. Kasantsev, and V. Yakovlev, 1985, "Interference of atoms in separated optical fields," J. Opt. Soc. Am. B 2, 1791.

Cheville, R. A., R. W. McGowan, and D. Grischkowsky, 1998, "Time resolved measurements which isolate the mechanisms responsible for terahertz glory scattering from dielectric spheres," Phys. Rev. Lett. 80, 269.

Chiao, R. Y., and A. D. Speliotopoulos, 2004, "Towards MIGO, the matter-wave interferometric gravitational-wave observatory, and the intersection of quantum mechanics with general relativity," J. Mod. Opt. 51, 861.

Chiao, R. Y., and Y. S. Wu, 1986, "Berry's phase in optical fibers," Phys. Rev. Lett. 57, 933.

Chikkatur, A. P., Y. Shin, A. E. Leanhardt, D. Kielpinski, E. Tsikata, T. L. Gustavson, D. E. Pritchard, and W. Ketterle, 2002, "A continuous source of Bose-Einstein condensed atoms," Science 296, 2193.

Chormaic, S. N., V. Wiedemann, Ch. Miniatura, J. Robert, S. Le Boiteux, V. Lorent, O. Gorceix, S. Feron, J. Reinhardt, and J. Baudon, 1993, "Longitudinal Stern-Gerlach atomic interferometry using velocity selected atomic beams," J. Phys. B 26, 1271.

Christ, M., A. Scholz, M. Schiffer, R. Deutschmann, and W. Ertmer, 1994, "Diffraction and reflection of a slow metastable neon beam by an evanescent light grating," Opt. Commun. 107, 211.

Chu, S., J. E. Bjorkholm, A. Ashkin, and A. Cable, 1986, "Experimental-observation of optically trapped atoms," Phys. Rev. Lett. 57, 314.

Chu, S., J. E. Bjorkholm, A. Ashkin, J. P. Gordon, and L. W. Hollberg, 1986, "Proposal for optically cooling atoms to temperatures of the order of $10^{-6} \mathrm{~K}$," Opt. Lett. 11, 73.

Chu, S., L. Hollberg, J. E. Bjorkholm, A. Cable, and A. Ashkin, 1985, "Three-dimensional viscous confinement and cooling of atoms by resonance radiation pressure," Phys. Rev. Lett. 55, 48.

Chu, S. Y., 1987, "Charge neutrality of atoms and magnetic monopoles,” Phys. Rev. Lett. 59, 1390.

Cimmino, A., G. I. Opat, A. G. Klein, H. Kaiser, S. A. Werner, M. Arif, and R. Clothier, 1989, "Observation of the topological Aharonov-Casher phase shift by neutron interferometry," Phys. Rev. Lett. 63, 380.

Clade, P., E. de Mirandes, M. Cadoret, S. Guellati-Khelifa, C. Schwob, F. Nez, L. Julien, and F. Biraben, 2006, "Determination of the fine structure constant based on Bloch oscillations of ultracold atoms in a vertical optical lattice," Phys. Rev. Lett. 96, 033001.

Clauser, J. F., 1988, "Ultra high sensitivity accelerometers and gyroscopes using neutral atom matter wave interferometry," Physica B \& C 151, 262.

Clauser, J. F., 1989, "Rotation, acceleration, and gravity sensors using quantum-mechanical matter-wave interferometry using neutral atoms and molecules," U.S. Patent No. $4,874,942$.

Clauser, J. F., 1997, De Broglie-Wave Interference of Small Rocks and Live Viruses (Kluwer Academic, Dordrecht).

Clauser, J. F., and S. F. Li, 1994a, "Heisenberg microscope decoherence atom interferometry," Phys. Rev. A 50, 2430.

Clauser, J. F., and S. F. Li, 1994b, "Talbot-vonLau atom interferometry with cold slow potassium," Phys. Rev. A 49, R2213. Clauser, J. F., and S. F. Li, 1997, "Generalized Talbot-Lau atom interferometry," in Atom Interferometry, edited by P. R. Berrman (Academic Press, San Diego), pp. 121-152.

Clauser, J. F., and M. Reinisch, 1992, "New theoretical and experimental results in Fresnel optics with applications to matter-wave and x-ray interferometry," Appl. Phys. B: Photophys. Laser Chem. 54, 380.

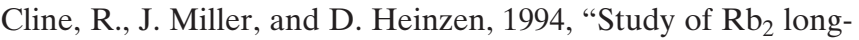
range states by high resolution photoassociation spectroscopy," Phys. Rev. Lett. 73, 632.

Cognet, L., V. Savalli, G. Z. K. Horvath, D. Holleville, R. Marani, N. Wiestbrook, C. I. Westbrook, and A. Aspect, 1998, "Atomic interference in grazing incidence diffraction from an evanescent wave mirror," Phys. Rev. Lett. 81, 5044.

Cohen, J. L., B. Dubetsky, P. R. Berman, and J. Schmiedmayer, 2000, "Filtered Talbot lens: Producing $\lambda / 2 n$-periodic atomic patterns with standing-wave fields having period $\lambda$," Phys. Rev. A 61, 033610.

Cohen-Tannoudji, C., 1998, "Manipulating atoms with photons," Phys. Scr. T76, 33.

Cohen-Tannoudji, C., and J. Dupont-Roc, 1972, "Experimental study of Zeeman light shifts in weak magnetic-fields," Phys. Rev. A 5, 968.

Colella, R., A. W. Overhauser, and S. A. Werner, 1975, "Observation of gravitationaly induced quantum interference," Phys. Rev. Lett. 34, 1472.

Colombe, Y., E. Knyazchyan, O. Morizot, B. Mercier, V. Lorent, and H. Perrin, 2004, "Ultracold atoms confined in rf-induced two-dimensional trapping potentials," Europhys. Lett. 67, 593.

Colombe, Y., B. Mercier, H. Perrin, and V. Lorent, 2005, "Diffraction of a Bose-Einstein condensate in the time domain," Phys. Rev. A 72, 061601(R).

Commins, E. D., 1991, "Berry geometric phase and motional fields,” Am. J. Phys. 59, 1077.

Cornell, E. A., and C. E. Wieman, 2002, "Nobel Lecture: BoseEinstein condensation in a dilute gas, the first 70 years and some recent experiments," Rev. Mod. Phys. 74, 875.

Cronin, A., and J. Perreault, 2004, "Phasor analysis of atom diffraction from a rotated material grating," Phys. Rev. A 70, 043607.

Cronin, A., L. Wang, and J. Perreault, 2005, "Limitations of nanotechnology for atom interferometry," e-print arXiv:physics/0508032.

Cronin, A. D., D. A. Kokorowski, T. D. Roberts, and D. E. Pritchard, 2003, "Controlled decoherence in an atom interferometer," Fortschr. Phys. 51, 313.

Dahan, M. B., E. Peik, J. Reichel, Y. Castin, and C. Salomon, 1996, "Bloch oscillations of atoms in an optical potential," Phys. Rev. Lett. 76, 4508.

Dalfovo, F., S. Giorgini, L. P. Pitaevskii, and S. Stringari, 1999, "Theory of Bose-Einstein condensation in trapped gases," Rev. Mod. Phys. 71, 463.

Dalibard, J., and C. Cohen-Tannoudji, 1985, "Dressed-atom approach to atomic motion in laser-light-the dipole force 
revisited," J. Opt. Soc. Am. B 2, 1707.

David, C., B. Nohammer, H. H. Solak, and E. Ziegler, 2002, "Differential x-ray phase contrast imaging using a shearing interferometer," Appl. Phys. Lett. 81, 3287.

Davisson, C., and L. H. Germer, 1927, "Diffraction of electrons by a crystal of nickel," Phys. Rev. 30, 705.

de Broglie, L., 1923, "Radiations-Ondes et quanta (Radiation-Waves and quanta)," Compt. Rend. 177, 507.

Degenhardt, C., H. Stoehr, C. Lisdat, G. Wilpers, H. Schnatz, B. Lipphardt, T. Nazarova, P. E. Pottie, U. Sterr, J. Helmcke, and F. Riehle, 2005, "Calcium optical frequency standard with ultracold atoms: Approaching $10^{-15}$ relative uncertainty," Phys. Rev. A 72, 062111.

Degenhardt, C., H. Stoehr, U. Sterr, F. Riehle, and C. Lisdat, 2004, "Wavelength-dependent ac stark shift of the ${ }^{1} S_{0^{-}}{ }^{3} P_{1}$ transition at $657 \mathrm{~nm}$ in Ca," Phys. Rev. A 70, 023414.

Dehuille, R., C. Champenois, M. Buchner, R. Mathevet, C. Rizzo, C. Robillard, and J. Vigué, 2001, in "Atom interferometry: Principles and applications to fundamental physics," Quantum Electrodynamics and Physics of the Vacuum, edited by G. Cantatore, AIP Conf. Proc. No. 564 (AIP, Melville, NY), pp. 192-199.

DeKieviet, M., D. Dubbers, S. Hafner, and F. Lang, 2000, "Atomic beam spin echo: Principle and surface science application," in Atomic and Molecular Beams; The State of the Art 2000, edited by R. Campargue (Springer, Heidelberg), pp. 161-174.

DeKieviet, M., D. Dubbers, M. Klein, U. Pieles, and C. Schmidt, 2000, "Design and performance of a highly efficient mass spectrometer for molecular beams," Rev. Sci. Instrum. 71, 2015.

DeKieviet, M., D. Dubbers, M. Klein, C. Schmidt, and M. Skrzipczyk, 1997, "Surface science using molecular beam spin echo," Surf. Sci. 377-379, 1112.

DeKieviet, M., D. Dubbers, C. Schmidt, D. Scholz, and U. Spinola, 1995, "3 He spin-echo-New atomic-beam technique for probing phenomena in the neV range," Phys. Rev. Lett. 75, 1919.

Dekker, H., 1981, "Classical and quantum-mechanics of the damped harmonic-oscillator," Phys. Rep. 80, 1.

Delhuille, R., C. Champenois, M. Buchner, L. Jozefowski, C. Rizzo, G. Trenec, and J. Vigué, 2002, "High-contrast MachZehnder lithium-atom interferometer in the Bragg regime," Appl. Phys. B: Lasers Opt. 74, 489.

Delhuille, R., A. Miffre, E. Lavallette, M. Buchner, C. Rizzo, G. Trenec, J. Vigué, H. J. Loesch, and J. P. Gauyacq, 2002, "Optimization of a Langmuir-Taylor detector for lithium," Rev. Sci. Instrum. 73, 2249.

Deng, L., 2006, “Theory of atom optics: Feynman's path integral approach," Fron. Phys. China 1, 47.

Deng, L., E. W. Hagley, J. Denschlag, J. E. Simsarian, M. Edwards, C. W. Clark, K. Helmerson, S. L. Rolston, and W. D. Phillips, 1999, "Temporal, matter-wave-dispersion Talbot effect," Phys. Rev. Lett. 83, 5407.

Denschlag, J., D. Cassettari, A. Chenet, S. Schneider, and J. Schmiedmayer, 1999, "A neutral atom and a wire: towards mesoscopic atom optics,” Appl. Phys. B: Lasers Opt. 69, 291. Derevianko, A., W. Johnson, M. Safranova, and J. Baab, 1999,

"High precision calculations of dispersion coefficients, static dipole polarizabilities, and atom-wall interaction constants for alkali-metal atoms," Phys. Rev. Lett. 82, 3589.

Derevianko, A., and S. G. Porsev, 2002, "Determination of lifetimes of $6 P_{J}$ levels and ground-state polarizability of Cs from the van der Waals coefficient $C_{6}$," Phys. Rev. A 65 , 053403 .

Deutschmann, R., W. Ertmer, and H. Wallis, 1993, "Reflection and diffraction of atomic de Broglie waves by an evanescent laser wave," Phys. Rev. A 47, 2169.

Dhirani, A. A., D. A. Kokorowski, R. A. Rubenstein, T. D. Hammond, B. Rohwedder, E. T. Smith, A. D. Roberts, and D. E. Pritchard, 1997, "Determining the density matrix of a molecular beam using a longitudinal matter wave interferometer," J. Mod. Opt. 44, 2583.

Dimopoulos, S., and A. A. Geraci, 2003, "Probing submicron forces by interferometry of Bose-Einstein condensed atoms," Phys. Rev. D 68, 124021.

Dimopoulos, S., P. Graham, J. Hogan, and M. Kasevich, 2007, "Testing general relativity with atom interferometry," Phys. Rev. Lett. 98, 111102.

Doak, R. B., R. E. Grisenti, S. Rehbein, G. Schmahl, J. P. Toennies, and C. Woll, 1999, "Towards realization of an atomic de Broglie microscope: Helium atom focusing using Fresnel zone plates," Phys. Rev. Lett. 83, 4229.

Dowling, J. P., C. P. Williams, and J. D. Franson, 1999, "Maxwell duality, Lorentz invariance, and topological phase," Phys. Rev. Lett. 83, 2486.

Drake, G., 1996, Ed., Atomic, Molecular, and Optical Physics Handbook (AIP, Woodbury, NY).

Dresden, M., and C. N. Yang, 1979, "Phase-shift in a rotating neutron or optical interferometer," Phys. Rev. D 20, 1846.

Druzhinina, V., and M. DeKieviet, 2003, "Experimental observation of quantum reflection far from threshold," Phys. Rev. Lett. 91, 193202.

Dubetsky, B., and M. A. Kasevich, 2006, "Atom interferometer as a selective sensor of rotation or gravity," Phys. Rev. A 74, 023615 .

Duren, R., and W. Groger, 1978, "Determination of Na-Ar ground-state potential from differential cross-section measurements," Chem. Phys. Lett. 56, 67.

Duren, R., G. P. Raabe, and C. Schlier, 1968, “Accurate fitting of interatomic potential from scattering: Alkali-rare gas systems," Z. Phys. 214, 410.

Durfee, D. S., and W. Ketterle, 1998, "Experimental studies of Bose-Einstein condensation," Opt. Express 2, 299.

Durr, S., T. Nonn, and G. Rempe, 1998a, "Fringe visibility and 'which-way' information in an atom interferometer," Phys. Rev. Lett. 81, 5705.

Durr, S., T. Nonn, and G. Rempe, 1998b, "Origin of quantummechanical complementarity probed by a 'which-way' experiment in an atom interferometer," Nature (London) 395, 33.

Durr, S., and G. Rempe, 1999, “Acceptance angle for Bragg reflection of atoms from a standing light wave," Phys. Rev. A 59, 1495.

Durr, S., and G. Rempe, 2000a, "Can wave-particle duality be based on the uncertainty relation?,” Am. J. Phys. 68, 1021.

Durr, S., and G. Rempe, 2000b, "Complementarity and quantum erasure in an atom interferometer," Opt. Commun. 179, 323.

Dylla, H. F., and J. G. King, 1973, "Neutrality of molecules by a new method," Phys. Rev. A 7, 1224.

Einstein, A. 1917, "On the quantum theory of radiation," Phys. Z. 18, 121.

Ekstrom, C., J. Schmiedmayer, M. Chapman, T. Hammond, and D. E. Pritchard, 1995, "Measurement of the electric polarizability of sodium with an atom interferometer," Phys. Rev. A 51, 3883. 
Englert, B. G., 1996, "Fringe visibility and which-way information: An inequality," Phys. Rev. Lett. 77, 2154.

Englert, B. G., J. Schwinger, and M. O. Scully, 1988, "Is spin coherence like humpty-dumpty. 1. Simplified treatment," Found. Phys. 18, 1045.

Englert, B. G., M. O. Scully, and H. Walther, 2000, "On mechanisms that enforce complementarity," J. Mod. Opt. 47, 2213.

Estermann, I., and A. Stern, 1930, "Beugung von molekularstrahlen (bending of molecular rays)," Z. Phys. 61, 95.

Estève, J., C. Gross, A. Weller, S. Giovanazzi, and M. K. Oberthaler, 2008, "Squeezing and entanglement in a Bose-Einstein condensate," Nature (London) 455, 1216.

Esteve, J., D. Stevens, C. Aussibal, N. Westbrook, A. Aspect, and C. I. Westbrook, 2004, "An atom interferometer for measuring loss of coherence from an atom mirror," Eur. Phys. J. D 31, 487.

Fabry, L., and A. Perot, 1899, "Théorie et applications d'une nouvelle méthode de Spectroscopie Interférentielle," Ann. Chim. Phys. 16, 115.

Fattori, M., C. D'Errico, G. Roati, M. Zaccanti, M. JonaLasinio, M. Modugno, M. Inguscio, and G. Modugno, 2008, "Atom interferometry with a weakly-interacting BoseEinstein condensate," Phys. Rev. Lett. 100, 080405.

Fattori, M., G. Lamporesi, T. Petelski, J. Stuhler, and G. M. Tino, 2003, "Towards an atom interferometric determination of the Newtonian gravitational constant," Phys. Lett. A 318, 184.

Fattori, M., G. Roati, B. Deissler, C. D'Errico, M. Zaccanti, M. Jona-Lasinio, L. Santos, M. Inguscio, and G. Modugno, 2008, "Magnetic dipolar interaction in a Bose-Einstein condensate atomic interferometer," Phys. Rev. Lett. 101, 190405.

Felber, J., G. Muller, R. Gähler, and R. Golub, 1990, "Time dependent neutron optics II: Diffraction in space and time," Physica B 162, 191.

Feldman, L. C., Mayer, D. W., and Picraux, S. T., 1982, Materials Analysis by Ion Channeling: Submicron Crystallography (Academic Press, New York).

Ferrari, G., N. Poli, F. Sorrentino, and G. M. Tino, 2006, "Long-lived Bloch oscillations with bosonic Sr atoms and application to gravity measurement at the micrometer scale," Phys. Rev. Lett. 97, 060402.

Feynman, R., R. Leighton, and M. Sands, 1965, The Feynman Lectures on Physics (Addison-Wesley, Reading, MA), Vols. 2 and 3.

Feynman, R. P., and A. R. Hibbs, 1965, Quantm Mechanics and Path Integrals (McGraw-Hill, New York).

Fiete, G. A., and E. J. Heller, 2003, "Semiclassical theory of coherence and decoherence," Phys. Rev. A 68, 022112.

Fixler, J. B., G. T. Foster, J. M. McGuirk, and M. A. Kasevich, 2007, "Atom interferometer measurement of the Newtonian constant of gravity," Science 315, 74.

Fizeau, H., 1853, "Sur les hypotheses relatives a l'ether luminex, et sur une experience qui parait demontrer que le movement des corps change la vitene avec aquelle la lumiere se propage dans leur interjeur," Ann. Phys. Chem. 165, 457.

Flanders, D. C., A. M. Hawryluk, and H. I. Smith, 1979, "Spatial period division-a new technique for exposing sub-micronlinewidth periodic and quasiperiodic patterns," J. Vac. Sci. Technol. 16, 1949.

Folman, R., P. Kruger, J. Schmiedmayer, J. Denschlag, and C. Henkel, 2002, "Microscopic atom optics: from wires to an atom chip," Adv. At., Mol., Opt. Phys. 48, 263.
Forrey, R. C., Y. Li, V. Kharchenko, and A. Dalgarno, 1996, "Index of refraction of noble gases for sodium matter-waves," Phys. Rev. A 54, 2180.

Forrey, R. C., Y. Li, V. Kharchenko, and A. Dalgarno, 1997, "Refining molecular potentials using atom interferometry," Phys. Rev. A 55, R3311.

Fortagh, J., and C. Zimmermann, 2007, "Magnetic microtraps for ultracold atoms," Rev. Mod. Phys. 79, 235.

Foster, G. T., J. B. Fixler, J. M. McGuirk, and M. A. Kasevich, 2002, "Method of phase extraction between coupled atom interferometers using ellipse-specific fitting," Opt. Lett. 27, 951.

Fray, S., C. A. Diez, T. W. Hansch, and M. Weitz, 2004, "Atomic interferometer with amplitude gratings of light and its applications to atom based tests of the equivalence principle," Phys. Rev. Lett. 93, 240404.

Freimund, D. L., K. Aflatooni, and H. Batelaan, 2001, "Observation of the Kapitza-Dirac effect," Nature (London) 413, 142.

Fujita, J., T. Kishimoto, S. Mitake, and F. Shimizu, 1999, "Electric-field modified atomic beam holography," J. Vac. Sci. Technol. B 17, 2953.

Fujita, J., S. Mitake, and F. Shimizu, 2000a, "Interferometric modulation of an atomic beam by an electric field: A phase hologram for atoms," Phys. Rev. Lett. 84, 4027.

Fujita, J., S. Mitake, and F. Shimizu, 2000b, "Real time pattern changing in atomic beam holography using phase shift by stark effect," J. Vac. Sci. Technol. B 18, 3590.

Fujita, J., M. Morinaga, T. Kishimoto, M. Yasuda, S. Matsui, and F. Shimizu, 1996, "Manipulation of an atomic beam by a computer-generated hologram," Nature (London) 380, 691.

Gallis, M. R., 1993, "Models for local Ohmic quantum dissipation," Phys. Rev. A 48, 1028.

Gallis, M. R., and G. N. Fleming, 1990, "Environmental and spontaneous localization," Phys. Rev. A 42, 38.

Garcia, O., B. Deissler, K. J. Hughes, J. M. Reeves, and C. A. Sackett, 2006, "Bose-Einstein-condensate interferometer with macroscopic arm separation," Phys. Rev. A 74, 031601(R).

Gati, R., M. Albiez, J. Folling, B. Hemmerling, and M. K. Oberthaler, 2006, "Realization of a single josephson junction for Bose-Einstein condensates," Appl. Phys. B: Lasers Opt. 82, 207.

Gati, R., J. Esteve, B. Hemmerling, T. B. Ottenstein, J. Appmeier, A. Weller, and M. K. Oberthaler, 2006, "A primary noise thermometer for ultracold bose gases," New J. Phys. 8, 189.

Gati, R., B. Hemmerling, J. Folling, M. Albiez, and M. K. Oberthaler, 2006, "Noise thermometry with two weakly coupled Bose-Einstein condensates," Phys. Rev. Lett. 96, 130404.

Gaubatz, U., P. Rudecki, S. Schiemann, and K. Bergmann, 1990, "Population transfer between molecular vibrational levels by stimulated Raman-scattering with partially overlapping laser fields-A new concept and experimental results," J. Chem. Phys. 92, 5363.

Geotsch, P., and R. Graham, 1996, "Decoherence by spontaneous emission in atomic-momentum transfer experiments," Phys. Rev. A 54, 5345.

Gerginov, V., K. Calkins, C. E. Tanner, J. J. McFerran, S. Diddams, A. Bartels, and L. Hollberg, 2006, "Optical frequency measurements of $6 s^{2} S_{1 / 2}-6 p{ }^{2} P_{1 / 2}\left(D_{2}\right)$ transitions in ${ }^{133} \mathrm{Cs}$ and their impact on the fine-structure constant," Phys. Rev. A 


\section{3, 032504.}

Gerlich, S., L. Hackermüller, K. Hornberger, A. Stibor, H. Ulbricht, M. Gring, F. Goldfarb, T. Savas, M. Müri, A. Mayor, and M. Arndt, 2007, "A Kapitza-Dirac-Talbot-Lau interferometer for highly polarizable molecules," Nat. Phys. 3, 711.

Gibble, K., 2006, "Difference between a photon's momentum and an atom's recoil," Phys. Rev. Lett. 97, 073002.

Giltner, D. M., R. W. McGowan, and S. A. Lee, 1995a, “Atom interferometer based on Bragg scattering from standing light waves," Phys. Rev. Lett. 75, 2638.

Giltner, D. M., R. W. McGowan, and S. A. Lee, 1995b, "Theoretical and experimental-study of the Bragg scattering of atoms from a standing light-wave," Phys. Rev. A 52, 3966.

Giulini, D., E. Joos, C. Kiefer, J. Kupsch, I. Stamatescu, and H. D. Zeh, 1996, Decoherence and the Appearance of a Classical World in Quantum Theory (Springer-Verlag, Heidelberg).

Godun, G. M., M. d'Arcy, G. Summy, and K. Burnett, 2001, "Prospects for atom interferometry," Contemp. Phys. 42, 77.

Gordon, J. P., and A. Ashkin, 1980, "Motion of atoms in a radiation trap," Phys. Rev. A 21, 1606.

Gorlitz, A., B. Schuh, and A. Weis, 1995, "Measurement of the Aharonov-Casher phase of aligned Rb atoms," Phys. Rev. A 51, R4305.

Gould, P., P. Martin, G. Ruff, R. Stoner, J. Picque, and D. Pritchard, 1991, "Momentum transfer to atoms by a standing light wave: Transition from diffraction to diffusion," Phys. Rev. A 43, 585.

Gould, P. L., G. A. Ruff, and D. E. Pritchard, 1986, "Diffraction of atoms by light-The near-resonant Kaptiza-Dirac effect," Phys. Rev. Lett. 56, 827.

Grisenti, R., W. Schöllkopf, J. Toennies, C. Hegerfeldt, and T. Kohler, 1999, "Determination of atom-surface van der Waals potentials from transmission-grating diffraction intensities," Phys. Rev. Lett. 83, 1755.

Grisenti, R. E., W. Schöllkopf, J. P. Toennies, G. C. Hegerfeldt, T. Kohler, and M. Stoll, 2000, "Determination of the bond length and binding energy of the helium dimer by diffraction from a transmission grating," Phys. Rev. Lett. 85, 2284.

Grisenti, R. E., W. Schöllkopf, J. P. Toennies, J. R. Manson, T. A. Savas, and H. I. Smith, 2000, "He-atom diffraction from nanostructure transmission gratings: The role of imperfections," Phys. Rev. A 61, 033608.

Gritsev, V., E. Altman, E. Demler, and A. Polkovnikov, 2006, "Full quantum distribution of contrast in interference experiments between interacting one dimensional bose liquids," Nat. Phys. 2, 705.

Grond, J., J. Schmiedmayer, and U. Hohenester, 2009, “Optimizing number squeezing when splitting a mesoscopic condensate," Phys. Rev. A 79, 021603.

Gross, B., A. Huber, M. Niering, M. Weitz, and T. W. Hansch, 1998, "Optical Ramsey spectroscopy of atomic hydrogen," Europhys. Lett. 44, 186.

Gundlach, J. H., and S. M. Merkowitz, 2000, "Measurement of Newton's constant using a torsion balance with angular acceleration feedback," Phys. Rev. Lett. 85, 2869.

Gunther, A., S. Kraft, M. Kemmler, D. Koelle, R. Kleiner, C. Zimmermann, and J. Fortagh, 2005, "Diffraction of a BoseEinstein condensate from a magnetic lattice on a microchip," Phys. Rev. Lett. 95, 170405.

Gunther, A., S. Kraft, C. Zimmermann, and J. Fortagh, 2007, "Atom interferometer based on phase coherent splitting of Bose-Einstein condensates with an integrated magnetic grating," Phys. Rev. Lett. 98, 140403.
Gupta, S., K. Dieckmann, Z. Hadzibabic, and D. E. Pritchard, 2002, "Contrast interferometry using Bose-Einstein condensates to measure $h / m$ and $\alpha$," Phys. Rev. Lett. 89, 140401.

Gupta, S., D. Kokorowski, R. Rubenstein, and W. Smith, 2001, "Longitudinal interferometry with atomic beams," Adv. At., Mol., Opt. Phys. 46, 243.

Gupta, S., A. E. Leanhardt, A. D. Cronin, and D. E. Pritchard, 2001, "Coherent manipulation of atoms with standing light waves," C. R. Acad. Sci., Ser IV: Phys., Astrophys. 2, 479.

Gustavson, T., P. Bouyer, and M. Kasevich, 1997, "Precision rotation measurements with an atom interferometer gyroscope," Phys. Rev. Lett. 78, 2046.

Gustavson, T. L., A. Landragin, and M. A. Kasevich, 2000, "Rotation sensing with a dual atom-interferometer Sagnac gyroscope," Class. Quantum Grav. 17, 2385.

Gustavsson, M., E. Haller, M. Mark, J. Danzl, G. RojasKopeinig, and H.-C. Naegerl, 2007, "Control of interactioninduced dephasing of bloch oscillations," Phys. Rev. Lett. 100, 080404.

Hackermuller, L., K. Hornberger, B. Brezger, A. Zeilinger, and M. Arndt, 2003, "Decoherence in a Talbot-Lau interferometer: the influence of molecular scattering," Appl. Phys. B: Lasers Opt. 77, 781.

Hackermuller, L., K. Hornberger, B. Brezger, A. Zeilinger, and M. Arndt, 2004, "Decoherence of matter waves by thermal emission of radiation," Nature (London) 427, 711.

Hackermuller, L., S. Uttenthaler, K. Hornberger, E. Reiger, B. Brezger, A. Zeilinger, and M. Arndt, 2003, "Wave nature of biomolecules and fluorofullerenes," Phys. Rev. Lett. 91, 090408 .

Hadzibabic, Z., S. Stock, B. Battelier, V. Bretin, and J. Dalibard, 2004, "Interference of an array of independent BoseEinstein condensates," Phys. Rev. Lett. 93, 180403.

Hagen, C. R., 1990, "Exact equivelance of spin-1/2 AharonovBohm and Aharonov-Casher effects," Phys. Rev. Lett. 64, 2347.

Hagley, E. W., L. Deng, M. Kozuma, J. Wen, K. Helmerson, S. L. Rolston, and W. D. Phillips, 1999, "A well-collimated quasi-continuous atom laser," Science 283, 1706.

Hajnal, J. V., K. Baldwin, P. Fisk, H. Bachor, and G. Opat, 1989, "Reflection and diffraction of sodium atoms by evanescent laser light fields," Opt. Commun. 73, 331.

Haldane, F., 1981, "Effective harmonic-fluid approach to lowenergy properties of one-dimensional quantum fluids," Phys. Rev. Lett. 47, 1840.

Hall, W. D., and J. C. Zorn, 1974, "Measurement of alkalimetal polarizabilities by deflection of a velocity-selected atomic beam," Phys. Rev. A 10, 1141.

Hamilton, W. A., A. G. Klein, G. I. Opat, and P. A. Timmins, 1987, "Neutron-diffraction by surface acoustic-waves," Phys. Rev. Lett. 58, 2770.

Hammond, T., M. Chapman, A. Lenef, J. Schmiedmayer, E. Smith, R. Rubenstein, D. Kokorowski, and D. Pritchard, 1997, "Matter-wave index of refraction, inertial sensing, and quantum decoherence in an atom interferometer," Braz. J. Phys. 27, 193.

Hammond, T., D. Pritchard, M. Chapman, L. A, and J. Schmiedmayer, 1995, "Multiplex velocity selection for precision matter-wave interferometry," Appl. Phys. B: Lasers Opt. 60, 193.

Han, Y. D., and I. G. Koh, 1992, "Topological nature of the Aharonov-Casher effect," Phys. Lett. A 167, 341.

Hanle, W., 1924, "Concerning the magnetic impact of polariza- 
tion of resonance fluorescence," Z. Phys. 30, 93.

Harber, D. M., J. M. Obrecht, J. M. McGuirk, and E. A. Cornell, 2005, "Measurement of the Casimir-Polder force through center of mass oscillations of a Bose-Einstein condensate," Phys. Rev. A 72, 033610.

Hasselbach, F., and M. Nicklaus, 1993, "Sagnac experiment with electrons-Observation of the rotational phase-shift of electron waves in vacuum," Phys. Rev. A 48, 143.

He, X. G., and B. H. J. McKellar, 1993, "Topological phase due to electric-dipole moment and magnetic monopole interaction," Phys. Rev. A 47, 3424.

Hegerfeldt, G. C., and T. Kohler, 1998, "Atomic versus molecular diffraction: Influence of breakups and finite size," Phys. Rev. A 57, 2021.

Hegerfeldt, G. C., and T. Kohler, 2000, "Deviations from classical optics in matter diffraction and determination of the size of weakly bound molecules," Phys. Rev. A 61, 023606.

Hegerfeldt, G. C., and M. Stoll, 2005, "Matter diffraction at oblique incidence: Higher resolution and the ${ }^{4} \mathrm{He}_{3}$ Efimov state," Phys. Rev. A 71, 033606.

Henkel, C., J. Y. Courtois, and A. Aspect, 1994, "Atomic diffraction by a thin phase grating," J. Phys. II 4, 1955.

Henkel, C., K. Molmer, R. Kaiser, N. Vansteenkiste, C. I. Westbrook, and A. Aspect, 1997, "Diffuse atomic reflection at a rough mirror," Phys. Rev. A 55, 1160.

Henkel, C., H. Wallis, N. Westbrook, C. I. Westbrook, A. Aspect, K. Sengstock, and W. Ertmer, 1999, "Theory of atomic diffraction from evanescent waves," Appl. Phys. B: Lasers Opt. 69, 277.

Heupel, T., H. Mei, N. Niering, B. Gross, M. Weitz, T. W. Hansch, and C. J. Bordé, 2002, "Hydrogen atom interferometer with short light pulses," Europhys. Lett. 57, 158.

Hils, T., J. Felber, R. Gahler, W. Glaser, R. Golub, K. Habicht, and P. Wille, 1998, "Matter-wave optics in the time domain: Results of a cold-neutron experiment," Phys. Rev. A 58, 4784.

Hinderthur, H., A. Pautz, V. Rieger, F. Ruschewitz, J. L. Peng, K. Sengstock, and W. Ertmer, 1997, “Three-beam atom interferometer," Phys. Rev. A 56, 2085.

Hinderthur, H., F. Ruschewitz, H. J. Lohe, S. Lechte, K. Sengstock, and W. Ertmer, 1999, "Time-domain high-finesse atom interferometry,” Phys. Rev. A 59, 2216.

Hinds, E., C. Vale, and M. Boshier, 2001, "Two-wire waveguide and interferometer for cold atoms," Phys. Rev. Lett. 86, 1462.

Hofferberth, S., I. Lesanovsky, B. Fischer, T. Schumm, and J. Schmiedmayer, 2007, "Non-equilibrium coherence dynamics in one-dimensional bose gases," Nature (London) 449, 324.

Hofferberth, S., I. Lesanovsky, B. Fischer, J. Verdu, and J. Schmiedmayer, 2006, "Rf dressed state potential for manipulation neutral atoms," Nat. Phys. 2, 710.

Hofferberth, S., I. Lesanovsky, T. Schumm, J. Schmiedmayer, A. Imambekov, V. Gritsev, and E. Demler, 2008, "Probing quantum and thermal noise," Nat. Phys. 4, 489.

Hohenester, U., P. Rekdal, A. Borzi, and J. Schmiedmayer, 2007, "Optimal quantum control of Bose-Einstein condensates in magnetic microtraps," Phys. Rev. A 75, 023602.

Hohm, U., 2000, "Polarizabilities of atoms and molecules, new insights into an old subject.," Vacuum 58, 117.

Holland, M., K. Burnett, C. Gardiner, J. I. Cirac, and P. Zoller, 1996, "Theory of an atom laser," Phys. Rev. A 54, R1757.

Holland, M., S. Marksteiner, P. Marte, and P. Zoller, 1996, "Measurement induced localization from spontaneous decay,” Phys. Rev. Lett. 76, 3683.
Hornberger, K., 2006, "Thermal limitation of far-field matterwave interference," Phys. Rev. A 73, 052102.

Hornberger, K., S. Gerlich, H. Ulbricht, L. Hackermüller, S. Nimmrichter, V. Goldt, O. Boltalina, and M. Arndt, 2009, "Theory and experimental verification of Kapitza-DiracTalbot-Lau interferometry,” New J. Phys. 11, 043032.

Hornberger, K., and J. E. Sipe, 2003, "Collisional decoherence reexamined," Phys. Rev. A 68, 012105.

Hornberger, K., J. E. Sipe, and M. Arndt, 2004, "Theory of decoherence in a matter wave Talbot-Lau interferometer," Phys. Rev. A 70, 053608.

Hornberger, K., S. Uttenthaler, B. Brezger, L. Hackermuller, M. Arndt, and A. Zeilinger, 2003, "Collisional decoherence observed in matter wave interferometry," Phys. Rev. Lett. 90, 160401.

Horne, M., I. Jex, and A. Zeilinger, 1999, "Schrodinger wave functions in strong periodic potentials with applications to atom optics," Phys. Rev. A 59, 2190.

Huber, A., B. Gross, M. Weitz, and T. W. Hansch, 1998, "Twophoton optical Ramsey spectroscopy of the $1 S-2 S$ transition in atomic hydrogen," Phys. Rev. A 58, R2631.

Hugbart, M., J. A. Retter, F. Gerbier, A. F. Veron, S. Richard, J. H. Thywissen, D. Clement, P. Bouyer, and A. Aspect, 2005, "Coherence length of an elongated condensate-A study by matter-wave interferometry," Eur. Phys. J. D 35, 155

Hughes, V. W., L. J. Fraser, and E. R. Carlson, 1988, "The electrical neutrality of atoms," Z. Phys. D: At., Mol. Clusters 10, 145 .

Inouye, S., T. Pfau, S. Gupta, A. Chikkatur, A. Gorlitz, D. Pritchard, and W. Ketterle, 1999, "Phase-coherent amplification of atomic matter waves," Nature (London) 402, 641.

Jaeger, G., A. Shimony, and L. Vaidman, 1995, "Two interferometric complementarities," Phys. Rev. A 51, 54.

Jahns, J., and A. W. Lohmann, 1979, "Lau effect (a diffraction experiment with incoherent illumination)," Opt. Commun. 28, 263.

Janicke, U., and M. Wilkens, 1996, "Prospects of matter wave amplification in the presence of a single photon," Europhys. Lett. 35, 561.

Jeltes, T., J. M. McNamara, W. Hogervorst, W. Vassen, V. Krachmalnicoff, M. Schellekens, A. Perrin, H. Chang, D. Boiron, A. Aspect, and C. I. Westbrook, 2007, "Comparison of the Hanbury Brown-Twiss effect for bosons and fermions," Nature (London) 445, 402.

Jentsch, C., T. Muller, E. M. Rasel, and W. Ertmer, 2004, "Hyper: A satellite mission in fundamental physics based on high precision atom interferometry," Gen. Relativ. Gravit. 36, 2197.

Jessen, P. S., C. Gerz, P. D. Lett, W. D. Phillips, S. L. Rolston, R. J. C. Spreeuw, and C. I. Westbrook, 1992, "Observation of quantized motion of Rb atoms in an optical-field," Phys. Rev. Lett. 69, 49.

Jo, G., Y. Shin, S. Will, T. Pasquini, M. Saba, W. Ketterle, D. Pritchard, M. Vengalattore, and M. M. Prentiss, 2007, "Long phase coherence time and number squeezing of two BoseEinstein condensates on an atom chip," Phys. Rev. Lett. 98, 030407.

Jo, G. B., J. H. Choi, C. Christensen, Y. R. Lee, T. Pasquini, W. Ketterle, and D. Pritchard, 2007, "Matter-wave interferometry with phase fluctuating Bose-Einstein condensates," Phys. Rev. Lett. 99, 240406.

Johnson, K. S., K. K. Berggren, A. Black, C. T. Black, A. P. Chu, N. H. Dekker, D. C. Ralph, J. H. Thywissen, R. 
Younkin, M. Tinkham, M. Prentiss, and G. M. Whitesides, 1996, "Using neutral metastable argon atoms and contamination lithography to form nanostructures in silicon, silicon dioxide, and gold," Appl. Phys. Lett. 69, 2773.

Johnson, K. S., J. H. Thywissen, N. H. Dekker, K. K. Berggren, A. P. Chu, R. Younkin, and M. Prentiss, 1998, "Localization of metastable atom beams with optical standing waves: Nanolithography at the Heisenberg limit," Science 280, 1583.

Joos, E., and H. D. Zeh, 1985, "The emergence of classical properties through interaction with the environment," $\mathrm{Z}$. Phys. B: Condens. Matter 59, 223.

Joy, D. C., D. E. Newbury, and D. L. Davidson, 1982, "Electron channeling patterns in the scanning electron microscope," J. Appl. Phys. 53, R81.

Jurgens, D., A. Greiner, R. Stutzle, A. Habenicht, E. T. Sligte, and M. K. Oberthaler, 2004, "Quantum features in atomic nanofabrication using exactly resonant standing waves," Phys. Rev. Lett. 93, 237402.

Kaiser, R., G. Labeyrie, A. Landragin, N. Vansteenkiste, C. Westbrook, J. Von-Zanthier, and A. Aspect, 1996, "Detecting bouncing atoms," Laser Phys. 6, 409.

Kaltenbaek, R., B. Blauensteiner, M. Zukowski, M. Aspelmeyer, and A. Zeilinger, 2006, "Experimental interference of independent photons," Phys. Rev. Lett. 96, 240502.

Kapitza, P. L., and P. A. M. Dirac, 1933, "The reflection of electrons from standing light waves," Proc. Cambridge Philos. Soc. 29, 297.

Kasevich, M., and S. Chu, 1991, "Atomic interferometry using stimulated Raman transitions," Phys. Rev. Lett. 67, 181.

Kasevich, M., and S. Chu, 1992, "Measurement of the gravitational acceleration of an atom with a light-pulse interferometer,” Appl. Phys. B: Photophys. Laser Chem. 54, 321.

Kasevich, M., D. S. Weiss, E. Riis, K. Moler, S. Kasapi, and S. Chu, 1991, "Atomic velocity selection using stimulated Raman transitions," Phys. Rev. Lett. 66, 2297.

Kasevich, M. A., 2002, "Coherence with atoms," Science 298, 1363.

Kasevich, M. A., E. Riis, S. Chu, and R. G. Devoe, 1989, "rf spectroscopy in an atomic fountain," Phys. Rev. Lett. 63, 612.

Katz, N., R. Ozeri, J. Steinhauer, N. Davidson, C. Tozzo, and F. Dalfovo, 2004, "High sensitivity phonon spectroscopy of Bose-Einstein condensates using matter-wave interference," Phys. Rev. Lett. 93, 220403.

Keith, D., M. Schattenburg, H. Smith, and D. Pritchard, 1988, "Diffraction of atoms by a transmission grating," Phys. Rev. Lett. 61, 1580.

Keith, D. W., C. R. Ekstrom, Q. A. Turchette, and D. Pritchard, 1991, “An interferometer for atoms," Phys. Rev. Lett. 66, 2693

Keller, C., M. K. Oberthaler, R. Abfalterer, S. Bernet, J. Schmiedmayer, and A. Zeilinger, 1997, "Tailored complex potentials and Friedel's law in atom optics," Phys. Rev. Lett. 79, 3327.

Keller, C., J. Schmiedmayer, and A. Zeilinger, 2000, "Requirements for coherent atom channeling," Opt. Commun. 179, 129.

Keller, C., J. Schmiedmayer, A. Zeilinger, T. Nonn, S. Durr, and G. Rempe, 1999, "Adiabatic following in standing-wave diffraction of atoms," Appl. Phys. B: Lasers Opt. 69, 303.

Ketterle, W., 2002, "Nobel Lecture: When atoms behave as waves: Bose-Einstein condensation and the atom laser," Rev. Mod. Phys. 74, 1131.

Keupp, J., A. Douillet, T. E. Mehlstaubler, N. Rehbein, E. M.
Rasel, and W. Ertmer, 2005, "A high-resolution RamseyBordé spectrometer for optical clocks based on cold $\mathrm{Mg}$ atoms," Eur. Phys. J. D 36, 289.

Kharchenko, P., J. F. Babb, and A. Dalgarno, 1997, "Longrange interactions of sodium atoms," Phys. Rev. A 55, 3566.

Kharchenko, V., and A. Dalgarno, 2001, "Refractive index for matter waves in ultracold gases," Phys. Rev. A 63, 023615.

Khare, V., and H. M. Nussenzveig, 1977, "Theory of glory," Phys. Rev. Lett. 38, 1279.

Kisters, T., K. Zeiske, F. Riehle, and J. Helmcke, 1994, "Highresolution spectroscopy with laser-cooled and trapped calcium atoms," Appl. Phys. B: Lasers Opt. 59, 89.

Kleckner, M. and A. Ron, 2001, "Decoherence of a pointer by a gas reservoir," Phys. Rev. A 63, 022110.

Kleppner, D., 1997, "A beginner's guide to the atom laser," Phys. Today 50, 11 .

Kohl, M., T. W. Hansch, and T. Esslinger, 2001, "Measuring the temporal coherence of an atom laser beam," Phys. Rev. Lett. 87, 160404.

Kohno, T., F. Shimizu, J. Fujita, and K. Shimizu, 2003, "Reflective double slit atom interferometer," J. Phys. Soc. Jpn. 72, 461.

Kokorowski, D., A. Cronin, T. Roberts, and D. Pritchard, 2001, "From single to multiple-photon decoherence in an atom interferometer," Phys. Rev. Lett. 86, 2191.

Kokorowski, D. A., A. Dhirani, T. D. Hammond, B. Rohwedder, R. A. Rubenstein, E. T. Smith, and D. E. Pritchard, 1998, "Fully quantized treatment of molecular beam resonance," Fortschr. Phys. 46, 849.

Kokorowski, D. A., T. D. Roberts, R. A. Rubenstein, E. T. Smith, and D. E. Pritchard, 2000, "Longitudinal atom interferometry," Fortschr. Phys. 48, 615.

Koolen, A., G. Jansen, K. Domen, H. Beijerinck, and K. van Leeuwen, 2002, "Large-angle adjustable coherent atomic beam splitter by Bragg scattering," Phys. Rev. A 65, 041601(R).

Kozuma, M., L. Deng, E. W. Hagley, J. Wen, R. Lutwak, K. Helmerson, S. L. Rolston, and W. D. Phillips, 1999, "Coherent splitting of Bose-Einstein condensed atoms with optically induced Bragg diffraction," Phys. Rev. Lett. 82, 871.

Kozuma, M., Y. Suzuki, Y. Torii, T. Sugiura, T. Kuga, E. W. Hagley, and L. Deng, 1999, "Phase-coherent amplification of matter waves," Science 286, 2309.

Kronik, L., I. Vasiliev, M. Jain, and J. R. Chelikowsky, 2001, "Ab initio structures and polarizabilities of sodium clusters," J. Chem. Phys. 115, 4322.

Kruger, E., W. Nistler, and W. Weirauch, 1995, "Determination of the fine-structure constant by a precise measurement of $h / m_{n}$, , Metrologia 32, 117.

Kuhnke, K., K. Kern, R. David, and G. Comsa, 1994, "Highefficiency molecular-beam ionization detector with short ionization region," Rev. Sci. Instrum. 65, 3458.

Kurtsiefer, C., T. Pfau, and J. Mlynek, 1997, "Measurement of the Wigner function of an ensemble of helium atoms," $\mathrm{Na}$ ture (London) 386, 150.

Landragin, A., L. Cognet, G. Z. K. Horvath, C. I. Westbrook, N. Westbrook, and A. Aspect, 1997, "A reflection grating for atoms at normal incidence," Europhys. Lett. 39, 485.

Landragin, A., G. Labeyrie, C. Henkel, R. Kaiser, N. Vansteenkiste, C. I. Westbrook, and A. Aspect, 1996, "Specular versus diffuse reflection of atoms from an evanescent-wave mirror," Opt. Lett. 21, 1591.

Lang, F., 1998, "Transversales spinecho an einem atomaren 
wasserstoffstrahl," Ph.D. thesis (Ruprecht-Karls-Universitat Heidelberg).

Langmuir, I., and K. H. Kingdon, 1925, "Thermionic effects caused by vapours of alkali metals," Proc. R. Soc. London, Ser. A 107, 61.

Lau, E., 1948, "Beugungserscheinungen an doppelrastern," Ann. Phys. 6, 417.

Leavitt, J. A., and F. A. Bills, 1969, "Single-slit diffraction pattern of a thermal atomic potassium beam," Am. J. Phys. 37, 905.

Le Coq, Y., J. A. Retter, S. Richard, A. Aspect, and P. Bouyer, 2006, "Coherent matter wave inertial sensors for precision measurements in space," Appl. Phys. B: Lasers Opt. 84, 627.

Lee, M. C., and U. E. Israelsson, 2003, "Fundamental physics research aboard the international space station," Physica B 329-333, 1649.

Lee, T. Y., 2001, "Quantum phases of electric and magnetic dipoles as special cases of the Aharonov-Bohm phase," Phys. Rev. A 64, 032107.

Lenef, A., T. Hammond, E. Smith, M. Chapman, R. Rubenstein, and D. Pritchard, 1997, "Rotation sensing with an atom interferometer," Phys. Rev. Lett. 78, 760.

Lennard-Jones, J. E., 1932, "Processes of adsorption and diffusion on solid surfaces," Trans. Faraday Soc. 28, 333.

Lesanovsky, I., S. Hofferberth, J. Schmiedmayer, and P. Schmelcher, 2006, "Manipulation of ultracold atoms in dressed adiabatic radio frequency potentials," Phys. Rev. A 74, 033619.

Lesanovsky, I., T. Schumm, S. Hofferberth, L. M. Andersson, P. Kruger, and J. Schmiedmayer, 2006, "Adiabatic radiofrequency potentials for the coherent manipulation of matter waves," Phys. Rev. A 73, 033619.

Letokhov, V. S., and V. G. Minogin, 1981, "Laser radiation pressure on free atoms," Phys. Rep. 73, 1.

Lett, P., K. Helmerson, W. Phillips, L. Ratliff, S. Rolston, and M. E. Wagshul, 1993, "Spectroscopy of $\mathrm{Na}_{2}$ by photoassociation of laser-cooled Na," Phys. Rev. Lett. 71, 2200.

Lidar, D., I. Chuang, and K. Whaley, 1998, "Decoherence-free subspaces for quantum computation," Phys. Rev. Lett. 81, 2594.

Lifshitz, 1956, "The theory of molecular attractive forces between solids," Sov. Phys. JETP 2, 73.

Lim, I. S., M. Pernpointner, M. L. Seth, J. K. Laerdahl, and P. Schwerdtfeger, 1999, "Relativistic coupled-cluster static dipole polarizabilities of the alkali metals from Li to element 119," Phys. Rev. A 60, 2822.

Lin Y. J., I. Teper, C. Chin, and V. Vuletic, 2004, "Impact of the Casimir-Polder potential and Johnson noise on Bose-Einstein condensate stability near surfaces," Phys. Rev. Lett. 92, 050404.

London, F., 1937, "The general theory of molecular forces," Trans. Faraday Soc. 33b, 8 .

Luis, A., and L. L. Sanchez-Soto, 1998, "Complementarity enforced by random classical phase kicks," Phys. Rev. Lett. 81, 4031.

Luo, F., C. F. Giese, and W. R. Gentry, 1996, "Direct measurement of the size of the helium dimer," J. Chem. Phys. 104, 1151.

Luo, F., G. C. McBane, G. S. Kim, C. F. Giese, and W. R. Gentry, 1993, "The weakest bond-Experimentalobservation of helium dimer," J. Chem. Phys. 98, 3564.

Malykin, G. B., 2000, "The Sagnac effect: Correct and incorrect explanations,” Usp. Fiz. Nauk 170, 1325.
Marani, R., L. Cognet, V. Savalli, N. Westbrook, C. I. Westbrook, and A. Aspect, 2000, "Using atomic interference to probe atom-surface interactions," Phys. Rev. A 61, 053402.

Marinelli, M., and G. Morpurgo, 1982, "Searches of fractionally charged-particles in matter with the magnetic levitation technique," Phys. Rep. 85, 161.

Marinelli, M., and G. Morpurgo, 1984, "The electric neutrality of matter-A summary," Phys. Lett. 137, 439.

Marinescu, M., A. Dalgarno, and J. Baab, 1997, "Retarded long-range potentials for the alkali-metal atoms and a perfectly conducting wall," Phys. Rev. A 55, 1530.

Maroulis, G., 2001, "On the static electric polarizability and hyperpolarizability of sodium. How good is the agreement between theory and experiment for the dipole polarizability?," Chem. Phys. Lett. 334, 207.

Martin, P. J., B. G. Oldaker, A. H. Miklich, and D. E. Pritchard, 1988, "Bragg scattering of atoms from a standing light wave," Phys. Rev. Lett. 60, 515.

Marton, L., 1952, Electron interferometer, Phys. Rev. 85, 1057. Marton, L., J. A. Simpson, and J. A. Suddeth, 1953, "Electron beam interferometer," Phys. Rev. 90, 490.

Marton, L., J. A. Simson, and J. A. Suddeth, 1954, "An electron interferometer," Rev. Sci. Instrum. 25, 1099.

Mathevet, R., R. Delhuille, and C. Rizzo, 2002, "Computation of the phase induced by non-Newtonian gravitational potentials in atom interferometry," Class. Quantum Grav. 19, L37. McClelland, J. J., S. B. Hill, M. Pichler, and R. J. Celotta, 2004, "Nanotechnology with atom optics," Sci. Technol. Adv. Mater. 5, 575.

McGuirk, J. M., G. T. Foster, J. B. Fixler, M. J. Snadden, and M. A. Kasevich, 2002, "Sensitive absolute-gravity gradiometry using atom interferometry," Phys. Rev. A 65, 033608.

McGuirk, J. M., D. M. Harber, J. M. Obrecht, and E. A. Cornell, 2004, "Alkali-metal adsorbate polarization on conducting and insulating surfaces probed with Bose-Einstein condensates," Phys. Rev. A 69, 062905.

Mei, M., T. W. Hansch, and M. Weitz, 2000, "Tensorial phases in multiple beam atomic interference," Phys. Rev. A 61, 020101(R).

Mei, M., and M. Weitz, 2001, "Controlled decoherence in multiple beam Ramsey interference,” Phys. Rev. Lett. 86, 559.

Meschede, D., and H. Metcalf, 2003, “Atomic nanofabrication: atomic deposition and lithography by laser and magnetic forces," J. Phys. D 36, R17.

Metcalf, H., and P. van der Stratten, 1999, Laser Cooling and Trapping (Springer, New York).

Mewes, M. O., M. R. Andrews, D. M. Kurn, D. S. Durfee, C. G. Townsend, and W. Ketterle, 1997, "Output coupler for Bose-Einstein condensed atoms," Phys. Rev. Lett. 78, 582.

Meystre, P., 2001, Atom Optics (Springer-Verlag, New York).

Mezei, F., 1993, "Study of slow dynamical processes by neutron-spin-echo," Int. J. Mod. Phys. B 7, 2885.

Michelson, A. A., 1881, "The relative motion of earth and ether," Am. J. Sci. 22, 20.

Michelson, A. A., 1925, "The effect of the earths rotation on the velocity of light part 1," Astrophys. J. 61, 137.

Miffre, A., M. Jacquey, M. Buchner, G. Trenec, and J. Vigué, 2006a, “Atom interferometry," Phys. Scr. 74, C15.

Miffre, A., M. Jacquey, M. Buchner, G. Trenec, and J. Vigué, 2006 b, "Atom interferometry measurement of the electric polarizability of lithium,” Eur. Phys. J. D 38, 353.

Miffre, A., M. Jacquey, M. Buchner, G. Trenec, and J. Vigué, 2006c, "Measurement of the electric polarizability of lithium 
by atom interferometry," Phys. Rev. A 73, 011603(R).

Migdall, A. L., J. V. Prodan, W. D. Phillips, T. H. Bergeman, and H. J. Metcalf, 1985, "First observation of magnetically trapped neutral atoms," Phys. Rev. Lett. 54, 2596.

Miller, J. D., R. Cline, and D. Heinzen, 1993, "Far-offresonance optical trapping of atoms," Phys. Rev. A 47, R4567.

Miniatura, C., F. Perales, G. Vassilev, J. Reinhardt, J. Robert, and J. Baudon, 1991, “A longitudinal Stern-Gerlach interferometer: the "beaded" atom," J. Phys. II 1, 425.

Miniatura, C., J. Robert, O. Gorceix, V. Lorent, S. Leboiteux, J. Reinhardt, and J. Baudon, 1992, "Atomic interferences and the topological phase,” Phys. Rev. Lett. 69, 261.

Minkowski, H., 1908, Nachr. Ges. Wiss. Goettingen, Math.Phys. K1. K1, 53.

Minkowski, H., 1910, "Fundamental equations for the electromagnetic processes in the moved bodies," Math. Ann. 68, 472.

Moerdijk, A. J., W. C. Stwalley, R. G. Hulet, and B. J. Verhaar, 1994, "Negative scattering length of ultracold ${ }^{7}$ Li gas," Phys. Rev. Lett. 72, 40.

Moerdijk, A., and B. Verhaar, 1994, "Prospects for BoseEinsten condensation in atomic ${ }^{7} \mathrm{Li}$ and ${ }^{23} \mathrm{Na}$," Phys. Rev. Lett. 73, 518.

Moharam, M. G., and L. Young, 1978, "Criterion for Bragg and Raman-Nath diffraction regimes," Appl. Opt. 17, 1757.

Mohr, P. J., and B. N. Taylor, 2005, "CODATA recommended values of the fundamental physical constants," Rev. Mod. Phys. 77, 1.

Mollenstedt, G., and H. Duker, 1955, "Frensnelscher interferenzversuch mit einem biprisma fur elektronenwellen," Naturwiss. 42, 41

Momose, A., S. Kawamoto, I. Koyama, Y. Hamaishi, K. Takai, and Y. Suzuki, 2003, "Demonstration of x-ray Talbot interferometry," Jpn. J. Appl. Phys., Part 2 42, L866.

Monroe, C., D. M. Meekhof, B. E. King, S. R. Jefferts, D. J. Itano, W. M. Wineland, and P. Gould, 1995, "Resolvedsideband Raman cooling of a bound atom to the 3D zeropoint energy," Phys. Rev. Lett. 75, 4011.

Morinaga, A., M. Nakamura, T. Kurosu, and N. Ito, 1996, "Phase shift induced from the dc stark effect in an atom interferometer comprised of four copropagating laser beams," Phys. Rev. A 54, R21.

Morinaga, A., F. Riehle, J. Ishikawa, and J. Helmcke, 1989, “A Ca optical frequency standard-Frequency stabilization by means of nonlinear Ramsey resonances," Appl. Phys. B: Photophys. Laser Chem. 48, 165.

Morinaga, A., T. Tako, and N. Ito, 1993, "Sensitive measurement of phase shifts due to the ac Stark effect in a Ca optical Ramsey interferometer," Phys. Rev. A 48, 1364.

Morinaga, M., M. Yasuda, T. Kishimoto, and F. Shimizu, 1996, "Holographic manipulation of a cold atomic beam," Phys. Rev. Lett. 77, 802.

Morsch, O., and M. Oberthaler, 2006, "Dynamics of BoseEinstein condensates in optical lattices," Rev. Mod. Phys. 78 , 179.

Moshinsky, M., 1952, "Diffraction in time," Phys. Rev. 88, 625. Moskowitz, P., P. Gould, S. Atlas, and D. Pritchard, 1983, "Diffraction of an atomic-beam by standing-wave radiation," Phys. Rev. Lett. 51, 370.

Muller, J. H., D. Bettermann, V. Rieger, K. Sengstock, U. Sterr, and W. Ertmer, 1995, "Topological phase-shift in a cold-atom interferometer," Appl. Phys. B: Lasers Opt. 60,
199.

Mun, J., P. Medley, G. Campbell, L. Marcassa, D. Pritchard, and W. Ketterle, 2007, "Phase diagram for a Bose-Einstein condensate moving in an optical lattice," Phys. Rev. Lett. 99, 150604.

Nairz, O., M. Arndt, and A. Zeilinger, 2003, "Quantum interference experiments with large molecules," Am. J. Phys. 71, 319.

Nairz, O., B. Brezger, M. Arndt, and A. Zeilinger, 2001, "Diffraction of complex molecules by structures made of light," Phys. Rev. Lett. 87, 160401.

Namiki, M., S. Pascazio, and H. Rauch, 1993, "Decoherence in neutron interferometry at low transmission probability," Phys. Lett. A 173, 87.

Negretti, A., and C. Henkel, 2004, "Enhanced phase sensitivity and soliton formation in an integrated bec interferometer," J. Phys. B 37, L385.

Neuhauser, W., M. Hohenstatt, P. Toschek, and H. Dehmelt, 1978, "Optical-sideband cooling of visible atom cloud confined in parabolic well," Phys. Rev. Lett. 41, 233.

Newton, R. G., 1966, Scattering Theory of Waves and Particles (McGraw-Hill, New York).

Nowak, S., C. Kurtsiefer, T. Pfau, and C. David, 1997, "Highorder Talbot fringes for atomic matter waves," Opt. Lett. 22, 1430.

Nowak, S., N. Stuhler, T. Pfau, and J. Mlynek, 1998, "Charged wire interferometer for atoms," Phys. Rev. Lett. 81, 5792.

Nussenzveig, H. M., 1979, "Complex angular-momentum theory of the rainbow and the glory," J. Opt. Soc. Am. 69, 1068.

Oates, C. W., F. Bondu, R. W. Fox, and L. Hollberg, 1999, “A diode-laser optical frequency standard based on laser-cooled Ca atoms: Sub-kilohertz spectroscopy by optical shelving detection," Eur. Phys. J. D 7, 449.

Oberthaler, M. K., R. Abfalterer, S. Bernet, C. Keller, J. Schmiedmayer, and A. Zeilinger, 1999, "Dynamical diffraction of atomic matter waves by crystals of light," Phys. Rev. A 60, 456.

Oberthaler, M. K., R. Abfalterer, S. Bernet, J. Schmiedmayer, and A. Zeilinger, 1996, "Atom waves in crystals of light," Phys. Rev. Lett. 77, 4980.

Oberthaler, M. K., S. Bernet, E. M. Rasel, J. Schmiedmayer, and A. Zeilinger, 1996, "Inertial sensing with classical atomic beams," Phys. Rev. A 54, 3165.

Oh, S., C. M. Ryu, and S. H. S. Salk, 1994, "Equivalence between Aharonov-Bohm and Aharonov-Casher effects and motive forces," Phys. Rev. A 50, 5320.

Ol'shanii, M. A., 1994, "Berry-phase-induced achromatic phase plate for atomic interferometry," Phys. Lett. A 186, 369.

Omnes, R., 1997, "General theory of the decoherence effect in quantum mechanics," Phys. Rev. A 56, 3383.

Orzel, C., A. K. Tuchman, M. L. Fenselau, M. Yasuda, and M. A. Kasevich, 2001, "Squeezed states in a Bose-Einstein condensate," Science 291, 2386.

Ovchinnikov, Y. B., J. H. Muller, M. R. Doery, E. J. D. Vredenbregt, K. Helmerson, S. L. Rolston, and W. D. Phillips, 1999, "Diffraction of a released Bose-Einstein condensate by a pulsed standing light wave," Phys. Rev. Lett. 83, 284.

Ozeri, R., N. Katz, J. Steinhauer, and N. Davidson, 2005, “Colloquium: Bulk Bogoliubov excitations in a Bose-Einstein condensate," Rev. Mod. Phys. 77, 187.

Panchos, J., 2003, private communication. 
Pasquini, T. A., M. Saba, G. B. Jo, Y. Shin, W. Ketterle, D. E. Pritchard, T. A. Savas, and N. Mulders, 2006, "Low velocity quantum reflection of Bose-Einstein condensates," Phys. Rev. Lett. 97, 093201.

Patorski, K., 1989, in Progress in Optics, XXVII, edited by E. Wolf (North-Holland, Amsterdam), pp. 1-108.

Paul, H., 1986, "Interference between independent photons," Rev. Mod. Phys. 58, 209.

Peierls, 1991, More Surprises in Theoretical Physics (Princeton University Press, Princeton, NJ).

Perreault, J. D., and A. D. Cronin, 2005, "Observation of atom wave phase shifts induced by van der Waals atom-surface interactions," Phys. Rev. Lett. 95, 133201.

Perreault, J. D., and A. D. Cronin, 2006, "Measurement of atomic diffraction phases induced by material gratings," Phys. Rev. A 73, 033610.

Perreault, J. D., A. D. Cronin, and T. A. Savas, 2005, "Using atomic diffraction of $\mathrm{Na}$ from material gratings to measure atom-surface interactions," Phys. Rev. A 71, 053612.

Peters, A., K. Y. Chung, and S. Chu, 1999, "Measurement of gravitational acceleration by dropping atoms," Nature (London) 400, 849.

Peters, A., K. Y. Chung, and S. Chu, 2001, "High-precision gravity measurements using atom interferometry," Metrologia 38, 25.

Peters, A., K. Y. Chung, B. Young, J. Hensley, and S. Chu, 1997, "Precision atom interferometry," Philos. Trans. R. Soc. London, Ser. A 355, 2223.

Pezze, L., L. Pitaevskii, A. Smerzi, S. Stringari, G. Modugno, E. de Mirandes, F. Ferlaino, H. Ott, G. Roati, and M. Inguscio, 2004, "Insulating behavior of a trapped ideal Fermi gas," Phys. Rev. Lett. 93, 120401.

Pezze, L., and A. Smerzi, 2006, "Phase sensitivity of a MachZehnder interferometer," Phys. Rev. A 73, 011801(R).

Pfau, T., S. Spalter, C. Kurtsiefer, C. Ekstrom, and J. Mlynek, 1994, "Loss of spatial coherence by a single spontaneous emission," Phys. Rev. Lett. 73, 1223.

Pfleegor, R. L., and L. Mandel, 1967, "Interference of independent photon beams," Phys. Rev. 159, 1084.

Polkovnikov, A., E. Altman, and E. Demler, 2006, "Interference between independent fluctuating condensates," Proc. Natl. Acad. Sci. U.S.A. 103, 6125.

Pritchard, D., 1989, "Matter wave optical systems in which an atomic beam intersects a diffraction grating at a grazing incidence," U.S. Patent No. 4,886,964.

Pritchard, D., M. Chapman, T. Hammond, D. Kokorowski, A. Lenef, R. Rubenstein, E. Smith, and J. Schmiedmayer, 1998, "Atom interferometers and atomic coherence," Fortschr. Phys. 46, 801.

Pritchard, D. E., 1983, "Cooling neutral atoms in a magnetic trap for precision spectroscopy," Phys. Rev. Lett. 51, 1336.

Pritchard, D. E., 1991, “Atom optics," in Atomic Physics, edited by C. Zorn and R. R. Lewis (AIP, New York), pp. 165174.

Pritchard, D. E., A. D. Cronin, S. Gupta, and D. A. Kokorowski, 2001, "Atom optics: Old ideas, current technology, and new results," Ann. Phys. 10, 35.

Quinn, T. J., C. C. Speake, S. J. Richman, R. S. Davis, and A. Picard, 2001, "A new determination of G using two methods," Phys. Rev. Lett. 87, 111101.

Raab, E. L., M. Prentiss, A. Cable, S. Chu, and D. E. Pritchard, 1987, "Trapping of neutral sodium atoms with radiation pressure," Phys. Rev. Lett. 59, 2631.
Rabi, I., J. Sacharias, S. Millman, and P. Kusch, 1938, "A new method for measuring nuclear magnetic moments," Phys. Rev. 53, 318.

Raman, C. V., and N. S. N. Nath, 1935, "The diffraction of light by high frequency sound waves," Proc. Indian Acad. Sci., Sect. A 2A, 406.

Ramsey, N., 1949, "A new molecular beam resonance method," Phys. Rev. 76, 996.

Ramsey, N., 1985, Molecular Beams (Oxford University Press). Rasel, E. M., M. K. Oberthaler, H. Batelaan, J. Schmiedmayer, and A. Zeilinger, 1995, "Atom wave interferometry with diffraction gratings of light," Phys. Rev. Lett. 75, 2633.

Rauch, H., and J. Summhammer, 1992, "Neutroninterferometer absorption experiments in the quantum limit," Phys. Rev. A 46, 7284.

Rauch, H., W. Treimer, and U. Bonse, 1974, "Test of a single crystal neutron interferometer," Phys. Lett. 47, 369.

Rauch, H., and S. A. Werner, 2000, Neutron Interferometry (Clarendon, New York).

Rayleigh, L., 1881, "On copying diffraction-gratings, and on some phenomena connected therewith," Philos. Mag. 11, 196. Reich, M., U. Sterr, and W. Ertmer, 1993, "Scheme for measuring a Berry phase in an atom interferometer," Phys. Rev. A 47, 2518 .

Reinisch, G., 1999, "Stern-Gerlach experiment as the pioneer-and probably the simplest—quantum entanglement test?," Phys. Lett. A 259, 427.

Rerat, M., M. Merawa, and B. Honvault-Bussery, 1998, “Ab initio calculations of dipole polarizabilities of $\mathrm{Na}$ and $\mathrm{K}$ in their $3^{2} D$-state and determination of long-range coefficients for $S+D$ molecular states of $\mathrm{Na}_{2}, \mathrm{~K}_{2}$, and $\mathrm{NaK}$," J. Chem. Phys. 109, 7246.

Richard, S., F. Gerbier, J. H. Thywissen, M. Hugbart, P. Bouyer, and A. Aspect, 2003, "Momentum spectroscopy of 1D phase fluctuations in Bose-Einstein condensates," Phys. Rev. Lett. 91, 010405.

Rieger, V., K. Sengstock, U. Sterr, J. H. Muller, and W. Ertmer, 1993, "Atom-interferometric determination of the dc-stark shift of the Mg-intercombination line," Opt. Commun. 99, 172.

Riehle, F., T. Kisters, A. Witte, J. Helmcke, and C. J. Bordé, 1991, "Optical Ramsey spectroscopy in a rotating frameSagnac effect in a matter-wave interferometer," Phys. Rev. Lett. 67, 177.

Riehle, F., H. Schnatz, G. Zinner, K. Zeiske, B. Lipphardt, and J. Helmcke, 1996, "Calcium optical frequency standard based on atom interferometry," Laser Phys. 6, 237.

Roati, G., E. De Mirandes, F. Ferlaino, H. Ott, G. Modugno, and M. Inguscio, 2004, "Atom interferometry with trapped Fermi gases," Phys. Rev. Lett. 92, 230402.

Robert, D., D. G. S. Guenther, and L. Bayvel, 2004, Eds., Encyclopedia of Modern Optics (Elsevier, Oxford).

Robert, J., C. Miniatura, S. L. Boiteux, J. Reinhardt, V. Bocvarski, and J. Baudon, 1991, "Atomic interferometery with metastable hydrogen atoms," Europhys. Lett. 16, 29.

Robert, J., C. Miniatura, O. Gorceix, S. Leboiteux, V. Lorent, J. Reinhardt, and J. Baudon, 1992, "Atomic quantum phase studies with a longitudinal Stern-Gerlach interferometer," J. Phys. II 2, 601.

Roberts, T. D., A. D. Cronin, D. A. Kokorowski, and D. E. Pritchard, 2002, "Glory oscillations in the index of refraction for matter waves," Phys. Rev. Lett. 89, 200406.

Roberts, T. D., A. D. Cronin, M. V. Tiberg, and D. E. Prit- 
chard, 2004, "Dispersion compensation for atom interferometry,” Phys. Rev. Lett. 92, 060405.

Rohwedder, B., 1999, "Atom optical elements based on nearfield grating sequences," Fortschr. Phys. 47, 883.

Rohwedder, B., 2000, "Efficient atomic beam splitters based on Talbot array illumination,” Appl. Phys. B: Lasers Opt. 70, 873.

Rohwedder, B., 2001, "Multiple atomic wave interferometry with standing-waves of light," Eur. Phys. J. D 13, 255.

Rohwedder, B., L. Davidovich, and N. Zagury, 1999, "Measuring the quantum state of an electromagnetic field using the atomic Talbot effect," Phys. Rev. A 60, 480.

Rohwedder, B., and M. F. Santos, 2000, "Atomic Talbot interferometry as a sensitive tool for cavity quantum electrodynamics," Phys. Rev. A 61, 023601.

Rolston, S. L., and W. D. Phillips, 2002, "Nonlinear and quantum atom optics," Nature (London) 416, 219.

Rosatzin, M., D. Suter, and J. Mlynek, 1990, "Light-shiftinduced spin echoes in a $J=1 / 2$ atomic ground-state," Phys. Rev. A 42, 1839.

Rothe, E. W., 1962, "Velocity dependence of the total cross section for the scattering of Li and K by Xe," Phys. Rev. 128, 659.

Roura, A., D. R. Brill, B. L. Hu, C. W. Misner, and W. D. Phillips, 2006, "Gravitational wave detectors based on matter wave interferometers (MIGO) are no better than laser interferometers (LIGO)," Phys. Rev. D 73, 084018.

Rubenstein, R. A., A. A. Dhirani, D. A. Kokorowski, T. D. Roberts, E. T. Smith, W. W. Smith, H. J. Bernstein, J. Lehner, S. Gupta, and D. E. Pritchard, 1999, "Search for off-diagonal density matrix elements for atoms in a supersonic beam," Phys. Rev. Lett. 82, 2018.

Rubenstein, R. A., D. A. Kokorowski, A. A. Dhirani, T. D Roberts, S. Gupta, J. Lehner, W. W. Smith, E. T. Smith, H. J. Bernstein, and D. E. Pritchard, 1999, "Measurement of the density matrix of a longitudinally modulated atomic beam," Phys. Rev. Lett. 83, 2285.

Ruostekoski, J., B. Kneer, W. P. Schleich, and G. Rempe, 2001 , "Interference of a Bose-Einstein condensate in a hard-wall trap: From the nonlinear Talbot effect to the formation of vorticity," Phys. Rev. A 63, 043613.

Ruschewitz, F., J. L. Peng, H. Hinderthur, N. Schaffrath, K. Sengstock, and W. Ertmer, 1998, "Sub-kilohertz optical spectroscopy with a time domain atom interferometer," Phys. Rev. Lett. 80, 3173.

Ryytty, P., M. Kaivola, and C. G. Aminoff, 1998, "Deflection of atoms by a pulsed standing wave: effects of laser field coherence," Quantum Semiclassic. Opt. 10, 545.

Saba, M., T. A. Pasquini, C. Sanner, Y. Shin, W. Ketterle, and D. E. Pritchard, 2005, "Light scattering to determine the relative phase of two Bose-Einstein condensates," Science 307, 1945.

Safronova, M. S., W. R. Johnson, and A. Derevianko, 1999, "Relativistic many-body calculations of energy levels, hyperfine constants, electric-dipole matrix elements, and static polarizabilities for alkali-metal atoms," Phys. Rev. A 60, 4476.

Sagnac, G., 1913a, "Luminous ether demonstrated by the effect of relative wind of ether in a uniform rotation of an interferometer," C. R. Hebd. Seances Acad. Sci. 157, 708.

Sagnac, G., 1913b, "The proof of the reality of luminous ether by the experiment of the turning interferograph," C. R. Hebd. Seances Acad. Sci. 157, 1410.
Sakurai, J., 1994, Modern Quantum Mechanics (AddisonWesley, Reading, MA).

Salomon, C., J. Dalibard, A. Aspect, H. Metcalf, and C. Cohen-Tannoudji, 1987, "Channeling atoms in a laser standing wave," Phys. Rev. Lett. 59, 1659.

Samuel, J., and R. Bhandari, 1988, "General setting for Berrys phase," Phys. Rev. Lett. 60, 2339.

Sangster, K., E. Hinds, S. Barnett, and E. Rils, 1993, "Measurement of the Aharonov-Casher phase in an atomic system," Phys. Rev. Lett. 71, 3641.

Sangster, K., E. A. Hinds, S. M. Barnett, E. Riis, and A. G. Sinclair, 1995, "Aharonov-Casher phase in an atomic system," Phys. Rev. A 51, 1776.

Sasagawa, G. S., W. Crawford, O. Eiken, S. Nooner, T. Stenvold, and M. A. Zumberge, 2003, "A new sea-floor gravimeter," Geophysics 68, 544.

Savalli, V., D. Stevens, J. Esteve, P. D. Featonby, V. Josse, N. Westbrook, C. I. Westbrook, and A. Aspect, 2002, "Specular reflection of matter waves from a rough mirror," Phys. Rev. Lett. 88, 250404.

Savas, T. A., M. L. Schattenburg, J. M. Carter, and H. I. Smith, 1996, "Large-area achromatic interferometric lithography for $100 \mathrm{~nm}$ period gratings and grids," J. Vac. Sci. Technol. B 14, 4167.

Savas, T. A., S. N. Shah, M. L. Schattenburg, J. M. Carter, and H. I. Smith, 1995, “Achromatic interferometric lithography for 100-nm-period gratings and grids," J. Vac. Sci. Technol. B 13, 2732.

Schattenburg, M. L., E. H. Anderson, and H. I. Smith, 1990, "X-ray/vuv transmission gratings for astrophysical and laboratory applications," Phys. Scr. 41, 13.

Schellekens, M., R. Hoppeler, A. Perrin, J. V. Gomes, D. Boiron, A. Aspect, and C. I. Westbrook, 2005, "Hanbury Brown Twiss effect for ultracold quantum gases," Science 310, 648.

Schiff, L. I., 1960, "Possible new experimental test of general relativity theory," Phys. Rev. Lett. 4, 215.

Schmiedmayer, J., M. Chapman, C. Ekstrom, T. D. Hammond, D. Kokorowski, A. Lenef, R. R. Rubenstein, E. Smith, and D. E. Pritchard, 1997, "Optics and interferometry with atoms and molecules," in Atom Interferometry, edited by P. R. Berrman (Academic Press, San Diego), pp. 1-83.

Schmiedmayer, J., M. Chapman, C. Ekstrom, T. Hammond, S. Wehinger, and D. Pritchard, 1995, "Index of refraction of various gases for sodium matter-waves," Phys. Rev. Lett. 74, 1043.

Schmiedmayer, J., C. Ekstrom, M. Chapman, T. Hammond, and D. Pritchard, 1994, "Magnetic coherences in atom interferometry,” J. Phys. II 4, 2029.

Schmiedmayer, J., C. R. Ekstrom, M. S. Chapman, T. D. Hammond, and D. E. Pritchard, 1993, in Fundamentals of Quantum Optics III, edited by F. Ehlotzky, Lecture Notes in Physics No. 420 (Springer-Verlag, Kuthai, Austria).

Schmiedmayer, J., and R. Folman, 2001, "Miniaturizing atom optics: from wires to atom chips," C. R. Acad. Sci., Ser IV: Phys., Astrophys. 2, 551.

Schöllkopf, W., and J. P. Toennies, 1994, "Non-destructive mass selection of small van der Waals clusters," Science 266, 1345.

Schöllkopf, W., and J. P. Toennies, 1996, "The nondestructive detection of the helium dimer and trimer," J. Chem. Phys. 104, 1155 .

Schöllkopf, W., J. P. Toennies, T. A. Savas, and H. I. Smith, 
1998, "A cluster size nanofilter with variable openings between 2 and 50 nm," J. Chem. Phys. 109, 9252.

Schumm, T., S. Hofferberth, L. M. Andersson, S. Wildermuth, S. Groth, I. Bar-Joseph, J. Schmiedmayer, and P. Kruger, 2005, "Matter-wave interferometry in a double well on an atom chip," Nat. Phys. 1, 57.

Schwinger, J., M. O. Scully, and B. G. Englert, 1988, "Is spin coherence like humpty-dumpty. 2. general-theory," Z. Phys. D: At., Mol. Clusters 10, 135.

Scoles, G., 1988, Ed., Atomic and Molecular Beam Methods (Oxford University Press, New York.).

Scully, M., and J. Dowling, 1993, "Quantum-noise limits to matter-wave interferometry," Phys. Rev. A 48, 3186(R).

Scully, M. O., B. G. Englert, and J. Schwinger, 1989, "Spin coherence and humpty-dumpty. III. The effects of observation," Phys. Rev. A 40, 1775.

Scully, M. O., B. G. Englert, and H. Walther, 1991, "Quantum optical tests of complementarity," Nature (London) 351, 111.

Search, C. P., and P. Meystre, 2003, "Noise limits in matterwave interferometry using degenerate quantum gases," Phys. Rev. A 67, 061601(R).

Seifert, W., Kaiserr, A. Aspect, and J. Mlynek, 1994, "Reflection of atoms from a dielectric wave-guide," Opt. Commun. 111, 566.

Sengstock, K., U. Sterr, G. Hennig, D. Bettermann, J. H. Muller, and W. Ertmer, 1993, "Optical Ramsey interferences on laser cooled and trapped atoms, detected by electron shelving," Opt. Commun. 103, 73.

Shevchenko, S. I., 1995, "Polarizable quantum-systems in crossed electric and magnetic-fields," Phys. Rev. Lett. 75, 3312.

Shimizu, F., 2001, "Specular reflection of very slow metastable neon atoms from a solid surface," Phys. Rev. Lett. 86, 987.

Shimizu, F., and J. Fujita, 2002a, "Giant quantum reflection of neon atoms from a ridged silicon surface," J. Phys. Soc. Jpn. 71, 5 .

Shimizu, F., and J. Fujita, 2002b, "Reflection-type hologram for atoms," Phys. Rev. Lett. 88, 123201.

Shimizu, F., K. Shimizu, and H. Takuma, 1992, "Stark phaseshift and deflection in the ultracold atomic interferometer," Jpn. J. Appl. Phys., Part 2 31, L436.

Shin, Y., M. Saba, T. A. Pasquini, W. Ketterle, D. E. Pritchard, and A. E. Leanhardt, 2004, "Atom interferometry with BoseEinstein condensates in a double-well potential," Phys. Rev. Lett. 92, 050405.

Shin, Y., C. Sanner, G. B. Jo, T. A. Pasquini, M. Saba, W. Ketterle, D. E. Pritchard, M. Vengalattore, and M. Prentiss, 2005, "Interference of Bose-Einstein condensates split with an atom chip," Phys. Rev. A 72, 021604(R).

Shinohara, K., T. Aoki, and A. Morinaga, 2002, "Scalar Aharonov-Bohm effect for ultracold atoms," Phys. Rev. A 66, 042106.

Shor, P., 1995, "Scheme for reducing decoherence in quantum computer memory," Phys. Rev. A 52, R2493.

Shull, C. G., 1968, "Observation of Pendellösung fringe structure in neutron diffraction," Phys. Rev. Lett. 21, 1585.

Simpson, J., 1954, "The theory of the three-cyrstal electron interferometer," Rev. Sci. Instrum. 25, 1105.

Sjoqvist, E., A. Pati, A. Ekert, J. Anandan, M. Ericsson, D. Oi, and V. Vedral, 2000, "Geometric phases for mixed states in interferometry," Phys. Rev. Lett. 85, 2845.

Smerzi, A., S. Fantoni, S. Giovanazzi, and S. R. Shenoy, 1997, "Quantum coherent atomic tunneling between two trapped
Bose-Einstein condensates," Phys. Rev. Lett. 79, 4950.

Smith, E. T., A. A. Dhirani, D. A. Kokorowski, R. A. Rubenstein, T. D. Roberts, H. Yao, and D. E. Pritchard, 1998, "Velocity rephased longitudinal momentum coherences with differentially detuned separated oscillatory fields," Phys. Rev. Lett. 81, 1996.

Snadden, M. J., J. M. McGuirk, P. Bouyer, K. G. Haritos, and M. A. Kasevich, 1998, "Measurement of the earth's gravity gradient with an atom interferometer-based gravity gradiometer," Phys. Rev. Lett. 81, 971.

Spavieri, G., 1999, "Quantum effect of the Aharonov-Bohm type for particles with an electric dipole moment," Phys. Rev. Lett. 82, 3932.

Spavieri, G., 2006, "Phase (or gauge) invariance and new fieldfree Aharonov-Bohm effects," Eur. Phys. J. D 37, 327.

Spruch, Y., and L. Tikochinsky, 1993, "Elementary approximate derivations of some retarded Casimir interactions involving one or two dielectric walls," Phys. Rev. A 48, 4213.

Stamper-Kurn, D. M., A. P. Chikkatur, A. Gorlitz, S. Gupta, S. Inouye, J. Stenger, D. E. Pritchard, and W. Ketterle, 2001, "Probing Bose-Einstein condensates with optical Bragg scattering," Int. J. Mod. Phys. B 15, 1621.

Steane, A., 1996, "Multiple-particle interference and quantum error correction,” Proc. R. Soc. London, Ser. A 452, 2551.

Steane, A., P. Szriftgiser, P. Desbiolles, and J. Dalibard, 1995, "Phase modulation of atomic de Broglie waves," Phys. Rev. Lett. 74, 4972.

Steinhauer, J., N. Katz, R. Ozeri, N. Davidson, C. Tozzo, and F. Dalfovo, 2003, "Bragg spectroscopy of the multibranch Bogoliubov spectrum of elongated Bose-Einstein condensates," Phys. Rev. Lett. 90, 060404.

Stenger, J., S. Inouye, A. P. Chikkatur, D. M. Stamper-Kurn, D. E. Pritchard, and W. Ketterle, 1999, "Bragg spectroscopy of a Bose-Einstein condensate," Phys. Rev. Lett. 82, 4569.

Stern, A., Y. Aharonov, and Y. Imry, 1990, "Phase uncertainty and loss of coherence: A general picture," Phys. Rev. A 41, 3436.

Sterr, U., C. Degenhardt, H. Stoehr, C. Lisdat, J. Schnatza, H. and Helmckea, F. Riehle, G. Wilpersc, C. Oates, and L. Hollberg, 2004, "The optical calcium frequency standards of PTB and NIST," C. R. Phys. 5, 845.

Steuernagel, O., and H. Paul, 1995, "Decoherence from spontaneous emission," Phys. Rev. A 52, R905.

Stoll, M., and T. Kohler, 2005, "Production of three-body Efimov molecules in an optical lattice," Phys. Rev. A 72, 022714.

Stoof, H., 1991, "Formation of the condensate in a dilute Bose gas," Phys. Rev. Lett. 66, 3148.

Storey, P., and C. Cohen-Tannoudji, 1994, "The Feynman pathintegral approach to atomic interferometry-A tutorial," J. Phys. II 4, 1999.

Stuhler, J., M. Fattori, T. Petelski, and G. M. Tino, 2003, "Magia-Using atom interferometry to determine the Newtonian gravitational constant," J. Opt. B: Quantum Semiclassical Opt. 5, S75.

Sukenik, C. I., M. G. Boshier, D. Cho, V. Sandoghdar, and E. A. Hinds, 1993, "Measurement of the Casimir-Polder force," Phys. Rev. Lett. 70, 560.

Sullivan, D. B., J. C. Bergquist, J. J. Bollinger, R. E. Drullinger, W. M. Itano, S. R. Jefferts, W. D. Lee, D. Meekhof, Parker, F. L. Walls, and D. J. Wineland, 2001, "Primary atomic frequency standards at NIST," J. Res. Natl. Inst. Stand. Technol. 106, 47.

Summhammer, J., G. Badurek, H. Rauch, U. Kischko, and A. 
Zeilinger, 1983, "Direct observation of fermion spin superpostion by neutron interferometry," Phys. Rev. A 27, 2523.

Summhammer, J., K. A. Hamacher, H. Kaiser, H. Weinfurter, and D. Jacobson, 1995, "Multiphoton exchange amplitudes observed by neutron interferometry," Phys. Rev. Lett. 75, 3206.

Summhammer, J., H. Rauch, and D. Tuppinger, 1987, "Stochastic and deterministic absorption in neutron-interference experiments," Phys. Rev. A 36, 4447.

Summhammer, J., H. Rauch, and D. Tuppinger, 1988, "Stochastic and deterministic attenuation of one beam in the neutron interferometer," Physica B \& C 151, 103.

Suter, D., G. C. Chingas, R. A. Harris, and A. Pines, 1987, "Berry phase in magnetic-resonance," Mol. Phys. 61, 1327.

Szriftgiser, P., D. GueryOdelin, M. Arndt, and J. Dalibard, 1996, "Atomic wave diffraction and interference using temporal slits," Phys. Rev. Lett. 77, 4.

Tan, S., and D. Walls, 1993, "Loss of coherence in interferometry," Phys. Rev. A 47, 4663.

Tang, K. T., and J. P. Toennies, 1977, "Simple theoreticalmodel for van der waals potential at intermediate distances. 1. spherically symmetric potentials," J. Chem. Phys. 66, 1496. Tanguy, C., S. Reynaud, M. Matsuoka, and C. Cohentannoudji, 1983, "Deflection profiles of a monoenergetic atomic-beam crossing a standing light-wave," Opt. Commun. 44, 249.

Tegmark, M., 1993, "Apparent wave function collapse caused by scattering," Found. Phys. Lett. 6, 571.

Tellinghuisen, J., A. Ragone, M. S. Kim, D. J. Auerbach, R. E. Smalley, L. Wharton, and D. H. Levy, 1979, "Dispersed fluorescence-spectrum of NaAr-ground and excited-state potential curves," J. Chem. Phys. 71, 1283.

Thakkar, A. J., and C. Lupinetti, 2005, "The polarizability of sodium: Theory and experiment reconciled," Chem. Phys. Lett. 402, 270.

Thirring, H., 1918, "The effect of rotating distant masses in the Einstein gravitational theory," Phys. Z. 19, 33.

Timp, G., R. E. Behringer, D. M. Tennant, J. E. Cunningham, M. Prentiss, and K. K. Berggren, 1992, "Using light as a lens for submicron, neutral-atom lithography," Phys. Rev. Lett. 69, 1636.

Tino, G. M., and F. Vetrano, 2007, "Is it possible to detect gravitational waves with atom interferometers?," Class. Quantum Grav. 24, 2167.

Toennies, J. P., 2001, "Novel spectroscopies using helium atoms clusters and droplets," Hinshelwood Lectures, Oxford, private communication.

Tonomura, A., 1999, Electron Holography (Springer-Verlag, Berlin).

Tonomura, A., L. Allard, G. Pozzi, J. D. C., and O. Y. A., 1999, Electron Holography (North-Holland, Amsterdam).

Torii, Y., Y. Suzuki, M. Kozuma, T. Sugiura, T. Kuga, L. Deng, and E. W. Hagley, 2000, "Mach-Zehnder Bragg interferometer for a Bose-Einstein condensate," Phys. Rev. A 61, 041602(R).

Turlapov, A., A. Tonyushkin, and T. Sleator, 2003, "Optical mask for laser-cooled atoms," Phys. Rev. A 68, 023408.

Turlapov, A., A. Tonyushkin, and T. Sleator, 2005, "Talbot-Lau effect for atomic de Broglie waves manipulated with light," Phys. Rev. A 71, 043612.

Tycko, R., 1987, “Adiabatic rotational splittings and Berrys phase in nuclear-quadrupole resonance," Phys. Rev. Lett. 58, 2281.

Unnikrishnan, C. S., and G. T. Gillies, 2004, "The electrical neutrality of atoms and of bulk matter," Metrologia 41, S125. Unruh, W., 1995, "Maintaining coherence in quantum computers,” Phys. Rev. A 51, 992.

Uys, H., J. D. Perreault, and A. D. Cronin, 2005, "Matter-wave decoherence due to a gas environment in an atom interferometer," Phys. Rev. Lett. 95, 150403.

Vacchini, B., 2004, "Quantum and classical features in the explanation of collisional decoherence," J. Mod. Opt. 51, 1025. Vigué, J., 1995, "Index of refraction of dilute matter in atomic interferometry," Phys. Rev. A 52, 3973.

Vuletic, V., C. Chin, A. J. Kerman, and S. Chu, 1998, "Degenerate Raman sideband cooling of trapped cesium atoms at very high atomic densities," Phys. Rev. Lett. 81, 5768.

Walker, T., and P. Feng, 1994, "Measurements of collisions between laser-cooled atoms," Adv. At., Mol., Opt. Phys. 34, 125. Wang, Y.-J., D., Anderson, V. Bright, E. Cornell, Q. Diot, T. Kishimoto, R. Prentiss, M. Saravanan, S. Segal, and S. Wu, 2005, "An atom Michelson interferometer on a chip using a Bose-Einstein condensate," Phys. Rev. Lett. 94, 090405.

Webb, C. L., R. M. Godun, G. S. Summy, M. K. Oberthaler, P. D. Featonby, C. J. Foot, and K. Burnett, 1999, "Measurement of Berry's phase using an atom interferometer," Phys. Rev. A 60, R1783.

Weel, M., I. Chan, S. Beattie, A. Kumarakrishnan, D. Gosset, and I. Yavin, 2006, "Effect of a magnetic field gradient and gravitational acceleration on a time-domain grating-echo interferometer," Phys. Rev. A 73, 063624.

Weiss, D., B. Young, and S. Chu, 1994, "Precision measurement of $\hbar / m_{C s}$ based on photon recoil using laser-cooled atoms and atomic interferometry," Appl. Phys. B: Lasers Opt. 59, 217.

Weiss, D. S., B. C. Young, and S. Chu, 1993, "Precisionmeasurement of the photon recoil of an atom using atomic interferometry," Phys. Rev. Lett. 70, 2706.

Weitkamp, T., B. Nohammer, A. Diaz, C. David, and E. Ziegler, 2005, "X-ray wavefront analysis and optics characterization with a grating interferometer," Appl. Phys. Lett. 86, 054101.

Weitz, M., T. Heupel, and T. W. Hansch, 1996, "Multiple beam atomic interferometer," Phys. Rev. Lett. 77, 2356.

Werner, S. A., J. L. Staudenmann, and R. Colella, 1979, "Effect of the earths rotation on the quantum mechanical phase of the neutron," Phys. Rev. Lett. 42, 1103.

Westbrook, N., C. I. Westbrook, A. Landragin, G. Labeyrie, L. Cognet, V. Savalli, G. Horvath, A. Aspect, C. Hendel, K. Moelmer, J. Y. Courtois, W. D. Phillips, 1998, "New physics with evanescent wave atomic mirrors: The van der Waals force and atomic diffraction," Phys. Scr. T78, 7.

Wheeler, J. A., and W. Zurek, 1983, Eds., Quantum Theory and Measurement (Princeton University Press, Princeton, $\mathrm{NJ})$.

Wicht, A., J. M. Hensley, E. Sarajlic, and S. Chu, 2002, “A preliminary measurement of the fine structure constant based on atom interferometry," Phys. Scr. T102, 82.

Wicht, A., E. Sarajlic, J. M. Hensley, and S. Chu, 2005, "Phase shifts in precision atom interferometry due to the localization of atoms and optical fields," Phys. Rev. A 72, 023602.

Wilczek, F., and A. Shapere, 1989, Eds., Geometric Phases in Physics, Advanced Series in Mathematical Physics, Vol. 5 (World Scientific, Singapore).

Wilkens, M., 1994, "Quantum phase of a moving dipole," Phys. Rev. Lett. 72, 5.

Wilkens, M., 1996, “de Broglie optics,” in Atomic, Molecular, 
and Optical Physics Handbook, edited by G. Drake (AIP, Woodbury, NY), Chap. 74.

Wilkens, M., E. Schumacher, and P. Meystre, 1991, "Transition from diffraction to diffusion in the near-resonant KaptizaDirac effect-A numerical approach," Opt. Commun. 86, 34.

Wilpers, G., T. Binnewies, C. Degenhardt, U. Sterr, J. Helmcke, and F. Riehle, 2002, "Optical clock with ultracold neutral atoms," Phys. Rev. Lett. 89, 230801.

Wineland, D. J., R. E. Drullinger, and F. L. Walls, 1978, "Radiation-pressure cooling of bound resonant absorbers," Phys. Rev. Lett. 40, 1639.

Wiseman, H., F. Harrison, M. J. Collett, S. Tan, D. Walls, and R. Killip, 1997, "Nonlocal momentum transfer in welcher Weg measurements," Phys. Rev. A 56, 55.

Wooters, W., and W. Zurek, 1979, "Complementarity in the double-slit experiment: Quantum non-separability and a quantitative statement of Bohr's principle," Phys. Rev. D 19, 473.

Wu, S., E. J. Su, and M. Prentiss, 2005, "Time domain de Broglie wave interferometry along a magnetic guide," Eur. Phys. J. D 35, 111.

Wu, S. J., Y. J. Wang, Q. Diot, and M. Prentiss, 2005, “Splitting matter waves using an optimized standing-wave light-pulse sequence," Phys. Rev. A 71, 043602.

Yanagimachi, S., M. Kajiro, M. Machiya, and A. Morinaga, 2002, "Direct measurement of the Aharonov-Casher phase and tensor stark polarizability using a calcium atomic polarization interferometer," Phys. Rev. A 65, 042104.

Yasuhara, M., T. Aoki, H. Narui, and A. Morinaga, 2005, "Measurement of Berry's phase for partial cycles using a time-domain atomic interferometer," IEEE Trans. Instrum. Meas. 54, 864.

Yasuda, M., and F. Shimizu, 1996, "Observation of two-atom correlation of an ultracold neon atomic beam," Phys. Rev. Lett. 77, 3090.

Yau, J. B., E. P. De Poortere, and M. Shayegan, 2002, "Aharonov-Bohm oscillations with spin: Evidence for Berry's phase," Phys. Rev. Lett. 88, 146801.

Young, B., M. Kasevich, and S. Chu, 1997, "Precision atom interferometery with light pulses," in Atom Interferometry, edited by P. R. Berrman (Academic Press, San Diego), pp. 363-406.

Zeilinger, A., 1986, in Fundamental Aspect of Quantum Theory, NATO Advanced Studies Institute, No. 144, Series B: Physics, edited by V. Gorini, and A. Frigerio (Plenum, New York), p. 311.

Zeilinger, A., R. Gahler, and M. A. Horne, 1991, "On the topological nature of the Aharonov-Casher effect," Phys. Lett. A 154, 93.

Zeiske, K., F. Riehle, G. Zinner, and J. Helmcke, 1994, "Measurement of the Aharonov-Casher phase in the atom interferometer," PTB-Mitt. 104, 339.

Zeiske, K., G. Zinner, F. Riehle, and J. Helmcke, 1995, “Atom interferometry in a static electric field: Measurement of the Aharonov-Casher phase," Appl. Phys. B: Lasers Opt. 60, 205.

Zener, C., 1934, "A theory of the electrical breakdown of solid dielectrics,” Proc. R. Soc. London, Ser. A 145, 523.

Zhou, F., and L. Spruch, 1995, "van der Waals and retardation (Casimir) interactions of an electron or an atom with multilayered walls," Phys. Rev. A 52, 297.

Zielonkowski, M., J. Steiger, U. Schunemann, M. DeKieviet, and R. Grimm, 1998, "Optically induced spin precession and echo in an atomic beam," Phys. Rev. A 58, 3993.

Zobay, O., and B. M. Garraway, 2001, "Two-dimensional atom trapping in field-induced adiabatic potentials," Phys. Rev. Lett. 86, 1195.

Zorn, J. C., G. E. Chamberlain, and V. W. Hughes, 1963, "Experimental limits for electron-proton charge difference and for charge of neutron," Phys. Rev. 129, 2566.

Zurek, W. H., 1991, "Decoherence and the transitions from quantum to classical," Phys. Today 44 (10), 36.

Zurek, W. H., 2003, "Decoherence, einselection, and the quantum origins of the classical," Rev. Mod. Phys. 75, 715. 